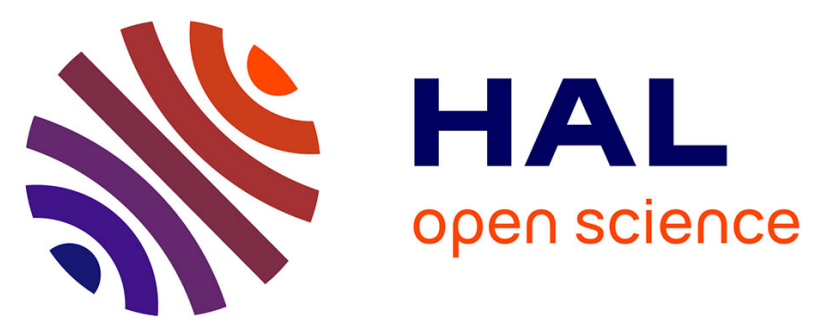

\title{
In vivo toxicity and genotoxicity of beauvericin and enniatins. Combined approach to study in vivo toxicity and genotoxicity of mycotoxins beauvericin (BEA) and enniatin B (ENNB)
}

Francesca Maranghi, Roberta Tassinari, Laura Narciso, Sabrina Tait, Cinzia Rocca, Gabriella Di Felice, Cinzia Butteroni, Silvia Corinti, Bianca Barletta, Eugenia Cordelli, et al.

\section{To cite this version:}

Francesca Maranghi, Roberta Tassinari, Laura Narciso, Sabrina Tait, Cinzia Rocca, et al.. In vivo toxicity and genotoxicity of beauvericin and enniatins. Combined approach to study in vivo toxicity and genotoxicity of mycotoxins beauvericin (BEA) and enniatin B (ENNB). [Research Report] 2018, pp.1-183. anses-01818576

\section{HAL Id: anses-01818576}

https://hal-anses.archives-ouvertes.fr/anses-01818576

Submitted on 19 Jun 2018

HAL is a multi-disciplinary open access archive for the deposit and dissemination of scientific research documents, whether they are published or not. The documents may come from teaching and research institutions in France or abroad, or from public or private research centers.
L'archive ouverte pluridisciplinaire HAL, est destinée au dépôt et à la diffusion de documents scientifiques de niveau recherche, publiés ou non, émanant des établissements d'enseignement et de recherche français ou étrangers, des laboratoires publics ou privés. 


\title{
In vivo toxicity and genotoxicity of beauvericin and enniatins. Combined approach to study in vivo toxicity and genotoxicity of mycotoxins beauvericin (BEA) and enniatin B (ENNB)
}

\author{
Francesca Maranghi ${ }^{a}$, Roberta Tassinari ${ }^{a}$, Laura Narciso ${ }^{a}$, Sabrina Tait ${ }^{a}$, Cinzia La Rocca ${ }^{a}$, \\ Gabriella Di Felice ${ }^{a}$, Cinzia Butteroni ${ }^{a}$, Silvia Corinti ${ }^{a}$, Bianca Barletta ${ }^{a}$, Eugenia Cordelli ${ }^{b}$, \\ Francesca Pacchierotti ${ }^{b}$, Patrizia Eleuteri ${ }^{b}$, Paola Villani ${ }^{b}$, Ludovic Le Hegarat ${ }^{c}$, Valérie \\ Fessard ${ }^{c}$, Océane Reale ${ }^{c}$ \\ ${ }^{a}$ Istituto Superiore di Sanità (ISS) Rome, Italy \\ ${ }^{b}$ Italian National Agency for New Technologies, Energy and Sustainable Economic Development \\ (ENEA) Rome, Italy \\ ${ }^{\mathrm{c}}$ French Agency for Food, Environmental and Occupational Health \& Safety (ANSES), Fougères,
} France

\begin{abstract}
Beauvericin (BEA) and Enniatins (ENN) are mycotoxins produced by Fusarium fungi detected in food and feed; there are insufficient data to establish their reference values. To evaluate BEA and ENN oral toxicity, an integrated approach was applied. Among ENN, Enniatin B (ENNB) was selected as test substance. The approach is composed by: i) in vitro and acute in vivo genotoxicity tests; ii) a repeated-dose oral toxicity study focused on genotoxic, immune, endocrine, nervous endpoints and the reproductive/developmental toxicity screening. For BEA, all the genotoxicity endpoints yielded negative results excluding Comet assay in duodenum and kidney after repeated doses. BEA immunotoxicity was observed in female mice, concentrated in number and functional activity of effector T cells in the spleen. Based on the repeated-dose BEA study, the No Observed Adverse Effect Level (NOAEL) for female mice is $1 \mathrm{mg} / \mathrm{kg}$ b.w. per day (increased thyroid pycnotic nuclei and endometrial hyperplasia). In males, the NOAEL is $0.1 \mathrm{mg} / \mathrm{kg}$ b.w. per day (reduced colloid and altered T4 serum levels). Maternal NOAEL is $0.1 \mathrm{mg} / \mathrm{kg}$ b.w. per day (increased thymus weight), developmental NOAEL is $10 \mathrm{mg} / \mathrm{kg}$ b.w. per day. For ENNB, the results support a genotoxic effect in bone marrow and liver cells after acute treatment, but not after repeated exposure. Immunotoxic ENNB effects were observed in both genders, suggestive of a suppressive/inhibiting activity particularly evident in males. Based on the repeated-dose ENNB study, the NOAEL for females is 0.18 $\mathrm{mg} / \mathrm{kg} \mathrm{b.w.} \mathrm{per} \mathrm{day} \mathrm{(histomorphometrical} \mathrm{effects} \mathrm{on} \mathrm{thymus,} \mathrm{uterus} \mathrm{and} \mathrm{spleen).} \mathrm{In} \mathrm{male} \mathrm{mice,} \mathrm{the}$ NOAEL is $1.8 \mathrm{mg} / \mathrm{kg}$ b.w. per day (enterocyte vacuolization in duodenum and increased Reactive Oxygen Species and reduced Glutathione brain levels). The maternal NOAEL is $1.8 \mathrm{mg} / \mathrm{kg} \mathrm{b.w}$. per day (decreased white pulp area and increased red/white pulp area ratio in spleen), developmental NOAEL is $18 \mathrm{mg} / \mathrm{kg}$ b.w. per day.

(C) Istituto Superiore di Sanità (ISS), Italian National Agency for New Technologies, Energy and Sustainable Economic Development (ENEA), and French Agency for Food, Environmental and Occupational Health \& Safety (ANSES), 2018
\end{abstract}

Key words: beauvericin, enniatin B, sub-chronic, acute, immunotoxicity, genotoxicity, histopathology

Question number: EFSA-Q-2015-00381

Correspondence: biocontam@efsa.europa.eu 
Disclaimer: The present document has been produced and adopted by the bodies identified above as authors. In accordance with Article 36 of Regulation (EC) No 178/2002, this task has been carried out exclusively by the authors in the context of a grant agreement between the European Food Safety Authority and the authors. The present document is published complying with the transparency principle to which the Authority is subject. It cannot be considered as an output adopted by the Authority. The European Food Safety Authority reserves its rights, view and position as regards the issues addressed and the conclusions reached in the present document, without prejudice to the rights of the authors.

Suggested citation: Istituto Superiore di Sanità (ISS), Italian National Agency for New Technologies, Energy and Sustainable Economic Development (ENEA) and French Agency for Food, Environmental and Occupational Health \& Safety (ANSES), 2018. In vivo toxicity and genotoxicity of beauvericin and enniatins. Combined approach to study in vivo toxicity and genotoxicity of mycotoxins beauvericin (BEA) and enniatin B (ENNB). EFSA supporting publication 2018:EN-1406. 183 pp. doi:10.2903/sp.efsa.2018.EN-1406

\section{ISSN: $2397-8325$}

(C) Istituto Superiore di Sanità (ISS), Italian National Agency for New Technologies, Energy and Sustainable Economic Development (ENEA), and French Agency for Food, Environmental and Occupational Health \& Safety (ANSES), 2018 


\section{Summary}

The structurally related mycotoxins Beauvericin (BEA) and Enniatins (ENN) are produced under moist and cool conditions mainly by Fusarium fungi and predominantly are found in cereal grains and their products. These two fusariotoxins are detected in a large proportion of analyzed food items like bread, rolls and pasta ( $80 \%$ for BEA and $63 \%$ for ENNB) and feed ( $79 \%$ for BEA and $32 \%$ for ENNB).

According to the European Food Safety Authority (EFSA) CONTAM Panel, there are insufficient data to establish a tolerable daily intake (TDI) and/or an acute reference dose (ARfD) for BEA or the sum of ENN. The Panel concluded that BEA and ENN acute exposure does not indicate concern for human health; concern might arise for chronic exposure but no firm conclusions could be drawn. Relevant in vivo toxicity data are needed to perform a human risk assessment.

In order to give information on BEA and ENNB oral toxicity in rodents, an integrated approach was applied, combining in vitro and in vivo acute oral genotoxicity tests with an oral repeated dose toxicity study in mice including the screening of reproductive and developmental toxicity. Among ENN, Enniatin B (ENNB) was selected as test substance. The approach was organized as follows:

a. Study on both the mycotoxins to determine the in vitro and in vivo genotoxicity upon acute or repeated dose exposure, according to Organisation for Economic Co-operation and Development (OECD) Test Guidelines (TG), EFSA opinion and international recommendations on genotoxicity testing.

b. $\quad$ Study on both the mycotoxins to evaluate: i) repeated dose oral toxicity in mice, focused on nervous, immune and endocrine endpoints, and ii) reproductive and developmental toxicity, on the basis of OECD TG and specific guidelines on immunotoxicity endpoints.

The BEA acute genotoxicity assessment by Comet assay in seven organs and the Micronucleus tests in bone marrow and colon indicated that no deoxyribonucleic acid (DNA) damage was observed, whatever the organs/tissues observed up to $200 \mathrm{mg} / \mathrm{kg} \mathrm{b.w.,} \mathrm{a} \mathrm{dose} \mathrm{that} \mathrm{largely} \mathrm{exceeds} \mathrm{the} \mathrm{highest}$ acute human exposure estimates for BEA. The in vitro genotoxicity studies were negative, only Micronucleus formation was observed at the highest concentration in terminally differentiated hepatic cells (HepaRG) cells. All the genotoxicity endpoints analyzed in the BEA repeated dose study yielded negative results with the exception of Comet assay in duodenum and kidney of male mice treated with the intermediate dose. Thus, overall, results suggest that BEA has a low genotoxic potential; further investigations may be necessary under a cautionary approach.

ENNB induced genotoxic effect in vivo after acute oral administration in male mice assessed by the Comet assay in the liver and bone marrow; no induction of DNA damage, gene mutations or chromosomal damage was observed after repeated exposure. Overall, the data support the hypothesis of a genotoxic hazard of ENNB.

As far as the effects of BEA and ENNB at tissue/biomarker level are concerned, BEA targeted thyroid, kidneys and reproductive systems in both sexes whereas spleen was affected in male mice only, and adrenals and duodenum in female mice only. Overall for these endpoints, taking into account also the severity of the effects, male mice seem more susceptible to BEA repeated oral exposure.

ENNB treatment affected spleen, brain and thyroid in both sexes, whereas thymus, kidneys, adrenals and reproductive system were affected in female mice only, and duodenum in male mice only. Overall for these endpoints, taking into account also the severity of the effects, female mice seem more susceptible to ENNB repeated oral exposure.

Thyroid and adrenals were target of both mycotoxins in male and/or female mice. These organs belong to the endocrine system and - in this respect - their impact on the whole organism, above all during critical lifestages, is well known. Although the reproductive/developmental screening with both BEA and ENNB didn't show any effect on the offspring, specific studies should be considered to verify potential endocrine disrupting mechanisms. 
As regards the global evaluation of immunotoxicity endpoints, it appears that the two mycotoxins induce different effects, mainly associated to the gender, the immune districts affected and to the number of endpoints significantly altered. In particular, the effects of BEA treatment on immune system appeared more evident in female mice in which they were concentrated in the spleen, with a prevalent positive action on the number and functional activity of effector $T$ cells. On the other hand, ENNB effects were observed in both genders, and they were more suggestive of a suppressive/inhibiting activity, particularly evident in male mice. Interestingly, parallel enhancing effects of the two mycotoxins on the innate compartment of the immune system (NO production by adherent spleen cells) were observed with the only exception of BEA-treated female mice in which an inhibitory action was recorded. Both the mycotoxins did not affect the red blood cells (RBC) and platelets (PLT) populations, suggesting the absence of effects on the haematopoietic and coagulation compartments, respectively. The effects of BEA and ENNB on the reduction of immunity-related blood cell populations (e.g., lymphocytes, granulocytes, monocyte-like) could be related to their myelotoxicity potential, observed in vitro on human hematopoietic progenitors. A possible explanation of the different pattern of effects induced by BEA and ENNB (central vs. peripheral effects, respectively) could be associated to the different metabolism of the two mycotoxins. In fact, despite their structural similarities, while for BEA no metabolites have been detected, ENNB metabolites have been found in liver and colon, thus potentially contributing in different modes to the biodistribution and the toxic effects.

Based on the repeated-dose BEA study, the No Observed Adverse Effect Level (NOAEL) for female mice is $1 \mathrm{mg} / \mathrm{kg}$ b.w. per day (increased thyroid pycnotic nuclei and endometrial hyperplasia). In male mice, the NOAEL is $0.1 \mathrm{mg} / \mathrm{kg}$ b.w. per day (reduced colloid and altered T4 serum levels). For the reproductive effects, the maternal NOAEL is $0.1 \mathrm{mg} / \mathrm{kg}$ b.w. per day (increased thymus weight). Developmental NOAEL is $10 \mathrm{mg} / \mathrm{kg}$ b.w. per day.

Based on the repeated-dose ENNB study, the NOAEL for female mice is $0.18 \mathrm{mg} / \mathrm{kg} \mathrm{b.w}$. per day (histomorphometrical effects on thymus, uterus and spleen). In male mice, the NOAEL is $1.8 \mathrm{mg} / \mathrm{kg}$ b.w. per day (enterocyte vacuolization in duodenum and increased Reactive Oxygen Species (ROS) and reduced Glutathione (GSH) brain levels). For the reproductive effects, the maternal NOAEL is 1.8 $\mathrm{mg} / \mathrm{kg} \mathrm{b.w.} \mathrm{per} \mathrm{day} \mathrm{(increased} \mathrm{white} \mathrm{pulp} \mathrm{area} \mathrm{and} \mathrm{decreased} \mathrm{red/white} \mathrm{pulp} \mathrm{area} \mathrm{ratio} \mathrm{in} \mathrm{spleen).}$ Developmental NOAEL is $18 \mathrm{mg} / \mathrm{kg}$ b.w. per day. 


\section{Table of contents}

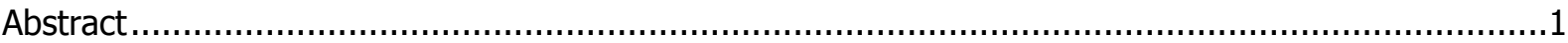

Summary

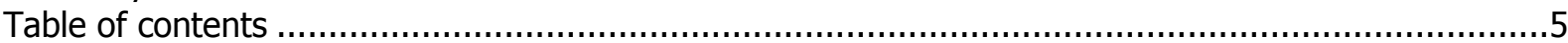

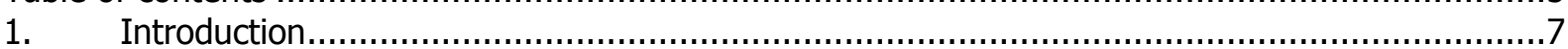

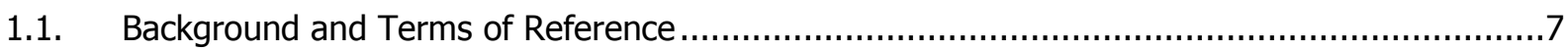

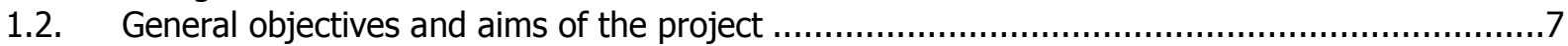

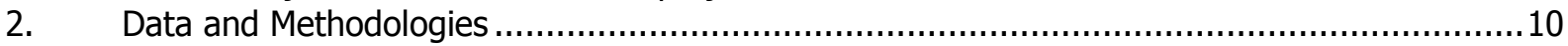

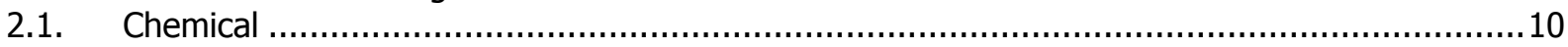

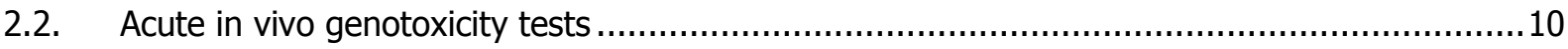

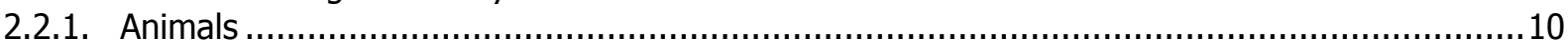

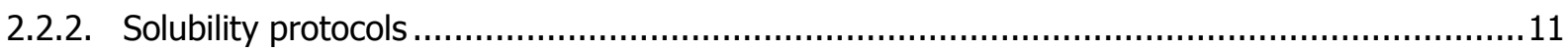

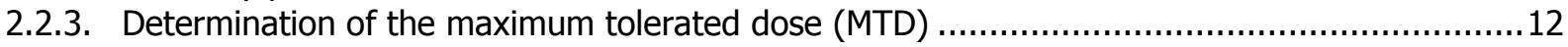

2.2.4. Oral administration for Comet assay and Micronucleus assay .......................................... 12

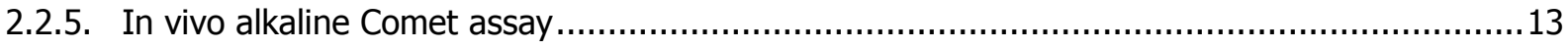

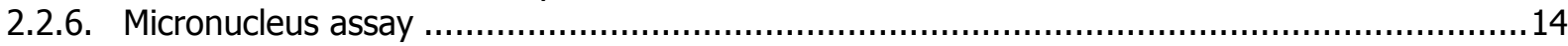

2.2.7. Immunohistochemistry protocol for $\mathrm{YH} 2 \mathrm{AX}$ detection in formalin-fixed paraffin-embedded

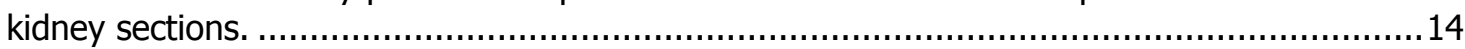

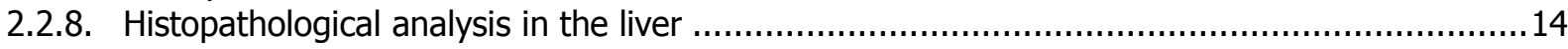

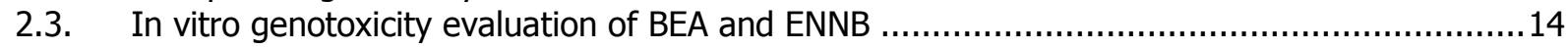

2.3.1. In vitro Micronucleus assay in undifferentiated human HepaRG cells ..............................15

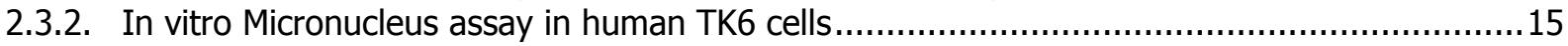

2.3.3. $\mathrm{yH} 2 \mathrm{AX} / \mathrm{pH} 3$ staining in undifferentiated HepaRG cells ..................................................16

2.4. Repeated dose oral toxicity study (OECD TG 407) and Reproductive/Developmental Toxicity

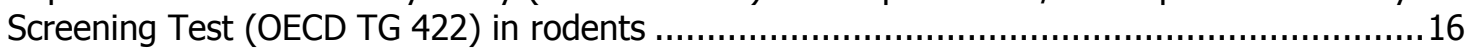

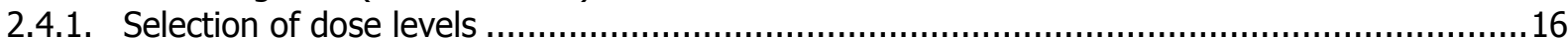

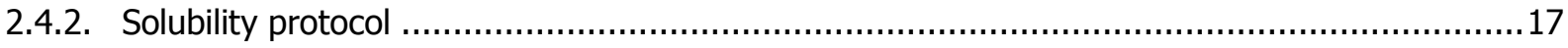

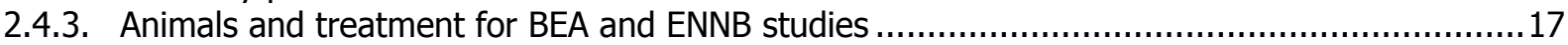

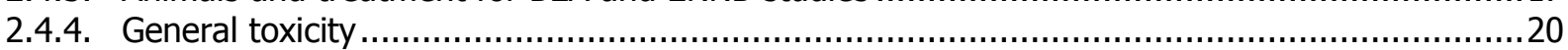

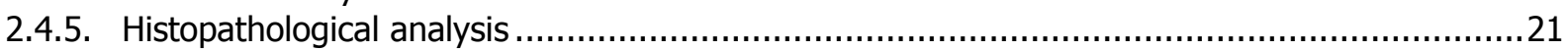

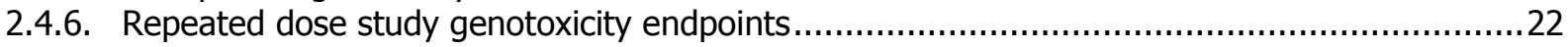

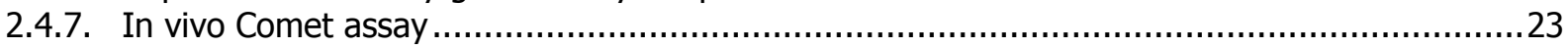

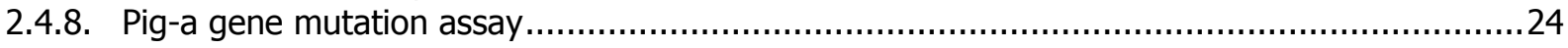

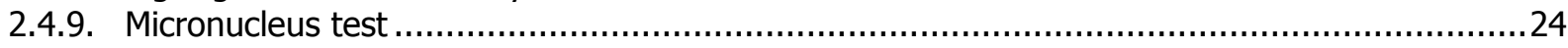

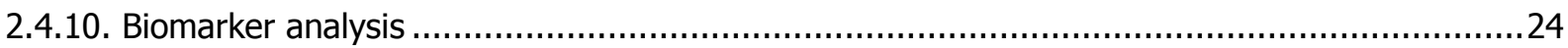

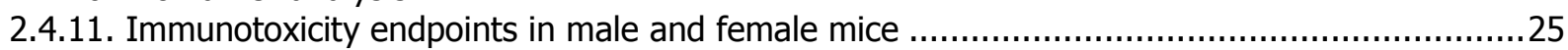

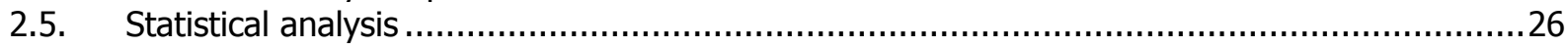

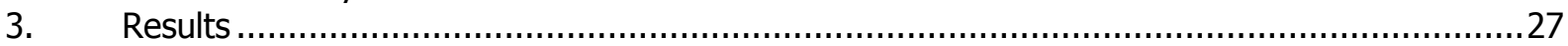

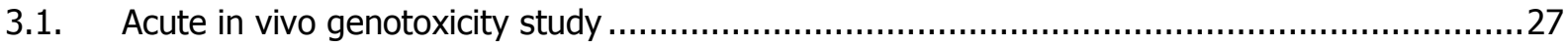

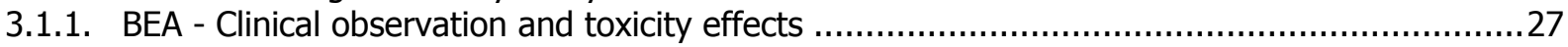

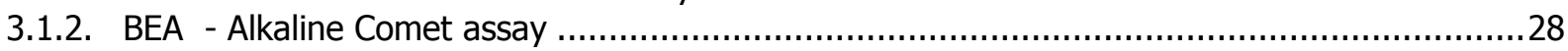

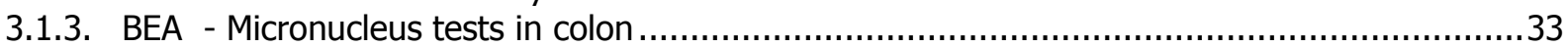

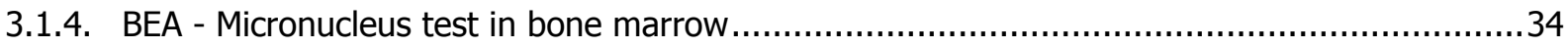

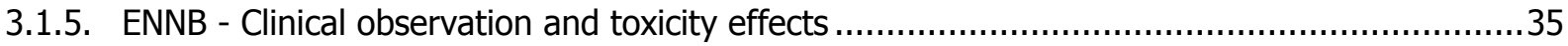

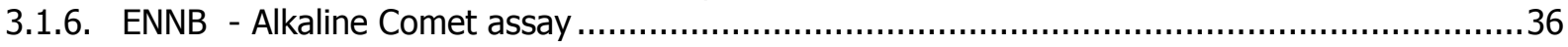

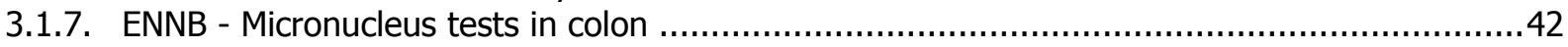

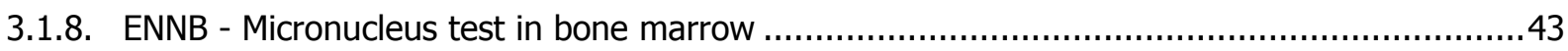

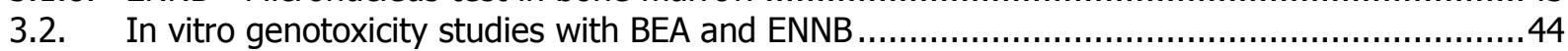

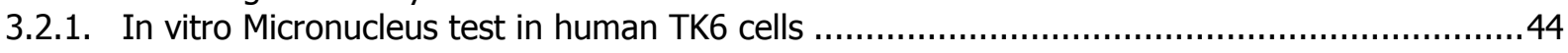

3.2.2. In vitro Micronucleus test in human undifferentiated HepaRG cells ....................................45

3.2.3. Results of the multiparametric genotoxicity tests with BEA and ENNB on proliferative HepaRG cells 


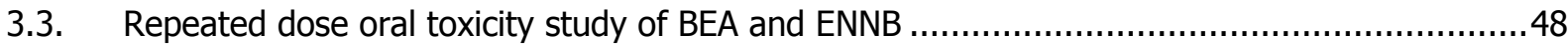

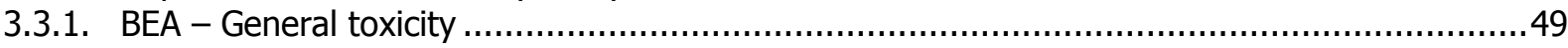

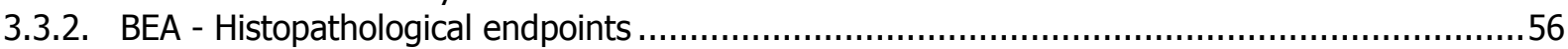

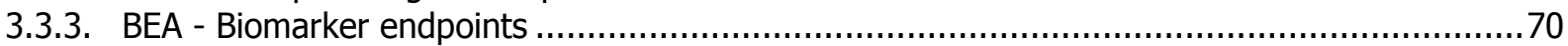

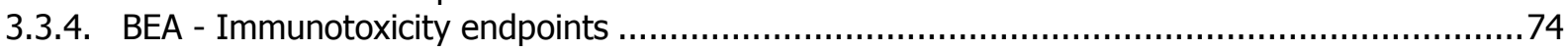

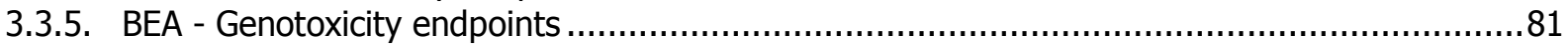

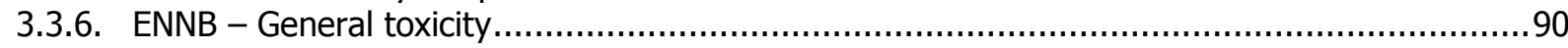

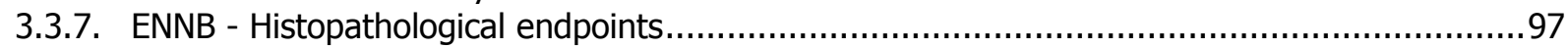

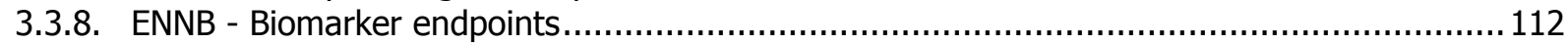

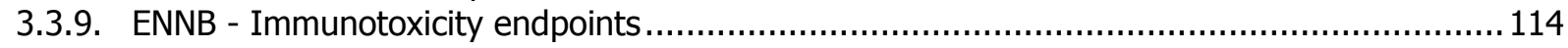

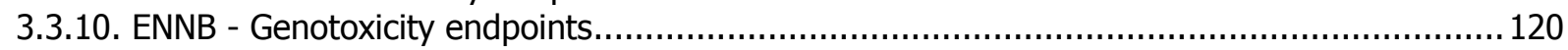

3.4. Reproduction/Developmental Toxicity Screening Test with BEA and ENNB .........................128

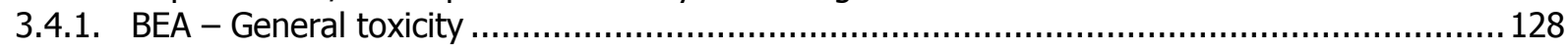

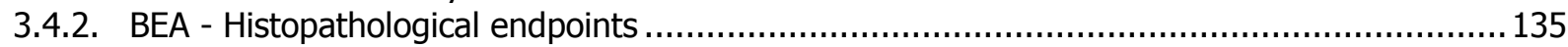

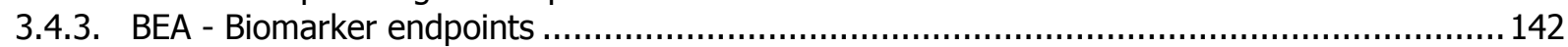

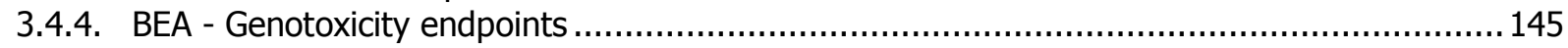

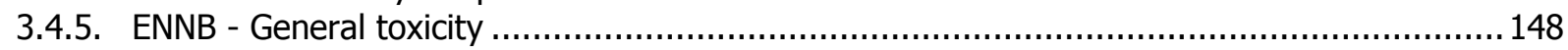

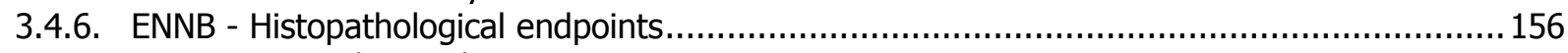

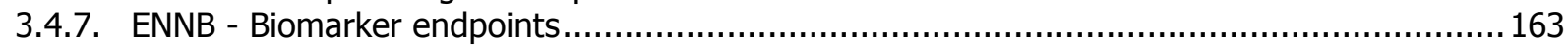

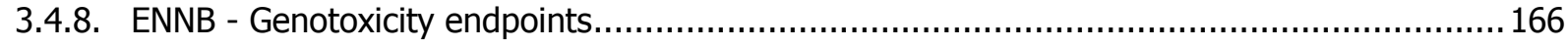

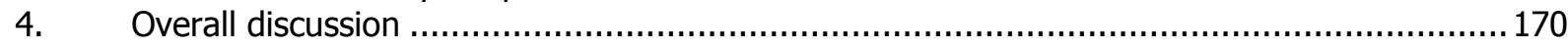

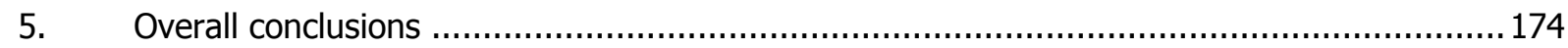

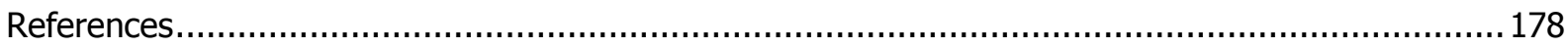

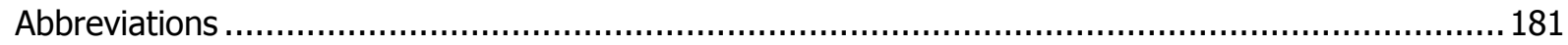

Appendix A - $\quad$ Timeline for in vivo genotoxicity tests integrated to repeat-dose toxicity study ..... 183

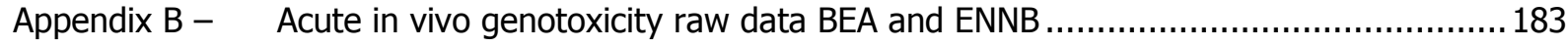

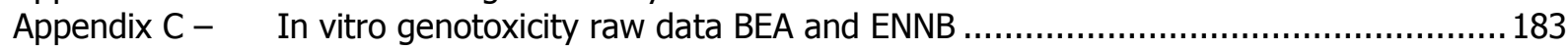

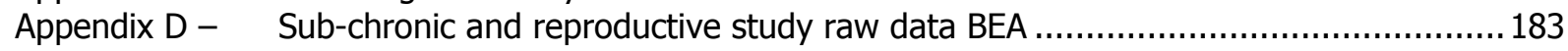

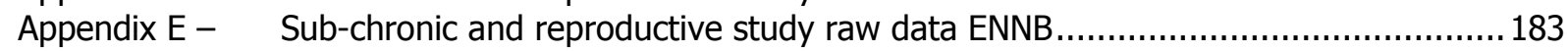




\section{Introduction}

\subsection{Background and Terms of Reference}

This grant was awarded by European Food Safety Authority (EFSA) to:

Beneficiary: Istituto Superiore di Sanità (ISS, Consortium leader), Italian National Agency for New Technologies, Energy and Sustainable Economic Development (ENEA, Partner 1), French Agency for Food, Environmental and Occupational Health and Safety (ANSES, Partner 2).

Grant title: In vivo toxicity and genotoxicity of beauvericin and enniatins

Grant number: GP/EFSA/BIOCONTAM/2015/02

Beauvericin (BEA) is a cyclic hexadepsipeptide that consists of alternating D-hydroxy-isovaleryl-(2hydroxy-3-methylbutanoic acid) and N-methylphenylalanine moieties. Enniatins (ENN) are structurally related mycotoxins representing a large group of cyclic hexadepsipeptides, whose chemical structure comprises D-hydroxy-isovaleryl-(2-hydroxy-3-methylbutanoic acid) and N-methylamino acid residues. So far, 29 naturally occurring ENN analogues have been identified but only ENN $A, A 1, B$ and B1, have been most frequently detected in food and feed (EFSA, 2014).

Among the four ENN identified by EFSA as the most representative for human exposure, Enniatin $B$ (ENNB) was selected as test substance in the proposed approach for the following reasons:

- $\quad$ the largest contribution to the chronic dietary exposure to the sum of ENNs is made by grains and grain-based products. The occurrence of ENN in grains showed that prevalence and concentration are the highest for both ENNB and B1, with the maximum value recorded in samples of Finnish spring wheat $(18,300 \mu \mathrm{g} / \mathrm{kg}$ ENNB);

- $\quad$ considering the occurrence of ENN in food products, in the dry pasta samples ENNB is predominant;

- in cereal-based baby foods, the incidence and concentration of ENNB was the highest, found in $28 \%$ of the samples, in concentrations up to $832 \mu \mathrm{g} / \mathrm{kg}$.

Although the EFSA CONTAM Panel concluded that the large margins obtained for acute exposure to BEA and ENN do not indicate concern for human health, concern is present for chronic exposure.

\subsection{General objectives and aims of the project}

In order to give information on the long term BEA and ENNB oral toxicity in rodents, an integrated approach, was applied.

The first phase of the project aimed at investigating the in vitro effects and acute in vivo genotoxic potential of BEA and ENNB. The Comet assay and Micronucleus (MN) test were performed according to OECD TG $489^{1}$ and $474^{2}$ in order to determine primary DNA and chromosome damage potentially induced by high oral exposure to the mycotoxins. An in vitro MN test was performed on human lymphoblast thymidine kinase heterozygote cell line (TK6) and on human undifferentiated hepatic (HepaRG) cells. In vitro multiparametric genotoxicity tests, aimed at distinguishing genotoxicants from nongenotoxicants, were also applied on proliferative HepaRG cells.

In the second phase, due to the BEA and ENN lack of data, a repeated dose oral toxicity study - on the basis of the OECD TG $407^{3}$ protocol - was performed, which provides information on the possible health hazards likely to arise from repeated exposure over a relatively limited period of time. The study investigated several endpoints related to general toxicity, tissue damage, biomarker alterations, immunotoxicity and genotoxicity.

\footnotetext{
${ }^{1}$ OECD, Test No. 489, 2014: In Vivo Mammalian Alkaline Comet Assay, OECD Publishing, Paris.

2 OECD, TEST No. 474, 2016: Mammalian Erythrocyte Micronucleus Test, OECD Publishing, Paris.

3 OECD, Test No. 407, 2008: Repeated Dose 28-day Oral Toxicity Study in Rodents, OECD Publishing, Paris.
} 
Within the repeated dose studies, each mycotoxin-treated animal cohort was coupled with a concurrent control (CTRL) group as follows: BEA with negative CTRL (olive oil alone) and ENNB with CTRL (olive oil + dimethyl sulfoxide (DMSO) 6\%), the vehicle used to dissolve ENNB. In the present approach, the treatment schedule of TG $407^{1}$ is extended, and the number of animals per group is increased, to include the screening of reproductive and developmental toxicity according to TG $422^{2}$. Parameters evaluated in the screening of reproductive and developmental toxicity are fully described in the OECD TG $422^{2}$ protocol.

The aim of the inclusion of general toxicity, biomarker and histopathological analysis endpoints are comprehensively described in the OECD TG $407^{1}$ protocol. Histomorphometrical quantitative analyses measure subtle differences at tissue level not immediately evident with traditional qualitative analysis. To evaluate potential effects of BEA and ENNB treatment on brain, oxidative stress status was measured using the Reactive Oxygen Species (ROS) and antioxidant glutathione (GSH) tissue levels.

Within the repeated dose toxicity study, the genotoxicity of the two mycotoxins was comprehensively assessed for gene mutation, DNA breakage, and chromosomal damage by employing the Pig-a gene mutation assay in peripheral blood, the Comet assay in different target organs (liver, duodenum, blood, kidney and testis/ovary cells), and the MN test in peripheral blood. The latter, which detects aneugenic and clastogenic chromosomal damage, is the most commonly applied in vivo assay and is among the genotoxicity tests officially approved as a regulatory test by OECD TG $474^{3}$. The flow cytometric version of the test was applied, since it allows the analysis of a high number of cells, and as such guarantees a higher level of reproducibility and sensitivity than the manual microscopy (Hayashi et al., 2007). Gene mutations potentially induced by chemicals in bone marrow erythroblasts were assessed in blood erythrocytes by the Pig-a assay. The rationale of the test is that mutations at the X-chromosome Pig-a locus can lead to loss of expression of glycosylphosphatidylinositol (GPI) anchored proteins on the surface of hematopoietic cells which can be identified by fluorescent antibody labelling and measured by flow cytometry. Although not yet accepted as a regulatory test, the Pig-a assay has already passed several validation steps demonstrating to be a robust and reproducible approach for in vivo genotoxicity assessment (Godin-Ethier et al, 2015; Gollapudi et al., 2015). It offers a valid alternative to the transgenic rodent mutation assay, which would require the use of a specific genetically modified mouse strain. One advantage of the Pig-a assay is the persistence of neutral Pig-a mutations induced in hematopoietic progenitor cells, resulting in the accumulation of mutants in the peripheral blood following repeated dosing (Miura et al., 2009). In the present study, a modified Pig-a protocol was applied in which the immunomagnetic separation of mutated red blood cells (RBC) or reticulocytes (RET), added as an additional step, increased the data acquisition rates and the number of cell equivalent evaluated per sample thereby increasing the sensitivity of the assay (Dertinger et al., 2011). The Comet assay was used for the detection of DNA strand breaks in single cells. The assay has been extensively validated in rodent liver cells (Rothfuss et al., 2010; OECD TG 489 ${ }^{4}$ ) but is applicable to any tissue from which analyzable single cell suspensions can be obtained thus allowing a variety of potential target tissues to be assessed for DNA damage. The combination of multiple tests, performed with minimal changes to the standard and regulatory recommended sampling times for the stand-alone assays, has been encouraged and successfully applied in genetic toxicology testing (Bowen et al., 2011; Gunther et al., 2014).

Immunotoxicity and immunological functions upon repeated dose exposure were studied by ex vivo/in vitro assays on treated and CTRL mice. Organs and tissues for the isolation and analysis of immune cell populations and/or soluble factors (blood, spleen, lymph nodes) were obtained from groups of 5 mice of both sexes at the end of the exposure period. Different parameters were evaluated, taking into account the relevant TG on immunotoxicity assessment by OECD and the International Conference on Harmonisation $(\mathrm{ICH})^{1}$ and the data already available in literature on immunomodulatory/immunotoxic effects of mycotoxins.

\footnotetext{
${ }^{1}$ OECD, Test No. 407, 2008: Repeated Dose 28-day Oral Toxicity Study in Rodents, OECD Publishing, Paris.

2 OECD, Test No. 422, 1996: Combined Repeated Dose Toxicity Study with the Reproduction/Developmental Toxicity Screening Test, OECD Publishing, Paris.

${ }^{3}$ OECD, Test No. 474, 2016: Mammalian Erythrocyte Micronucleus Test, OECD Publishing, Paris

${ }^{4}$ OECD, Test No. 489, 2014: In Vivo Mammalian Alkaline Comet Assay, OECD Publishing, Paris.
} 
Blood counts were performed soon after blood collection and sacrifice, with particular attention to hematologic changes such as leukocytopenia/leukocytosis, granulocytopenia/granulocytosis or lymphopenia/lymphocytosis. Serum samples were prepared and stored to evaluate levels of serum immunoglobulins (IgA, IgG, IgM isotypes).

Alterations in immune system organ weights and histopathology (e.g. changes in thymus, spleen, lymph nodes) were recorded. According to the suggestions of the ICH S8 Guideline ${ }^{1}$ the lymph node draining the exposure site (mesenteric lymph node, MLN) was selected to evaluate local effects.

Cytokine production (Interferon- $y$, Interleukin-10) in response to in vitro polyclonal stimulation was studied in spleen cells from treated and CTRL mice, as well as the distribution of T lymphocyte subpopulations (cluster of differentiation (CD)3+, CD3+/CD4+, CD3+/CD8+ number and frequency), on the basis of data observed in an in vivo study of oral exposure to ENN A (Juan et al, 2014). Enzyme-linked immunosorbent assay (ELISA) assays on culture supernatants and fluorescentactivated cell sorter (FACS) analysis on freshly isolated spleen cells were applied, respectively.

The contribution of the innate immunity compartment to potential inflammatory responses induced by oral exposure to tested materials was studied by evaluating Nitric Oxide (NO) levels in supernatants of adherent spleen cells stimulated with Lipid Poly-Saccharide (LPS).

As far as possible, the immunological and immunotoxicity studies were performed in compliance with Good Laboratory Practice (GLP), even if it is recognized that some specific assays may not fully comply with GLP.

The data derived from this experimental approach using the OECD TG allowed to extensively characterize the toxicity potential for both mycotoxins, to obtain indications about the dose response relationships and to determine NOAELs.

\footnotetext{
${ }^{1}$ ICH Topic S8 Immunotoxicity Studies for Human Pharmaceuticals. NOTE FOR GUIDANCE ON IMMUNOTOXICITY STUDIES FOR HUMAN PHARMACEUTICALS (CHMP/167/235/2004) 


\section{Data and Methodologies}

\subsection{Chemical}

Beauvericin (BEA, CAS Reg. 26048-05-5) and Enniatin B (ENNB, CAS Reg. 917-13-5) were purchased from VinciBiochem (Vinci, Italy) and produced by AdipoGen LIFE SCIENCES (Lot No A00759) and Cayman Chemical (Lots No 482831 and 494285), purity 97\% (impurities not determined by the manufacturer) and $\geq 99 \%$, respectively. Dimethyl sulfoxide (CAS Reg. No. 67-68-5, DMSO) and olive oil were purchased from Sigma-Aldrich (Italy). Methylmethanesulfonate (MMS) (CAS No 66-27-3) was purchased from Sigma-Aldrich (Italy) or Acros Organics (Belgium). N-ethyl-N-nitrosourea (ENU) (CAS $\mathrm{N}^{\circ}$ 753-73-9) was purchased from Sigma-Aldrich (Italy). Agarose, Triton-X100, Ethylenediamincetetraacetic acid (EDTA) was purchased from Acros Organics (Belgium). Sodium pentobartital and Hank's balanced salt solution (HBSS) were purchased, respectively, from Ceva (France) and Invitrogen (France).

\subsection{Acute in vivo genotoxicity tests}

Two independent in vivo genotoxicity studies were performed on male mice with both the mycotoxins. In fact, due to technical problems in the bone marrow MN test, a second in vivo study was performed to fill the gap. In the first experiment, in vivo alkaline Comet assay in 7 organs/tissues coupled with the colon MN test was performed after 3 oral administrations of BEA or 2 oral administrations of ENNB. In the second experiment, only 2 oral administrations of mycotoxins were done, followed by the in vivo Comet assay in liver and bone marrow and $\mathrm{MN}$ test in bone marrow.

The general overview of the genotoxicity studies, in vivo and in vitro is shown in Figure 1.

\subsubsection{Animals}

Male CD-1 mice, average body weight (b.w.) around $18 \mathrm{~g}$, were obtained from Charles River Laboratories (Italy). Starting with an acclimatizing period of five days under a 12-hours light/dark cycle in an air condition room at $20-23^{\circ} \mathrm{C}$, mice were fed ad libitum. 


\section{Task 1 acute in vivo genotoxicity tests in mice and in vitro genotoxicity tests}

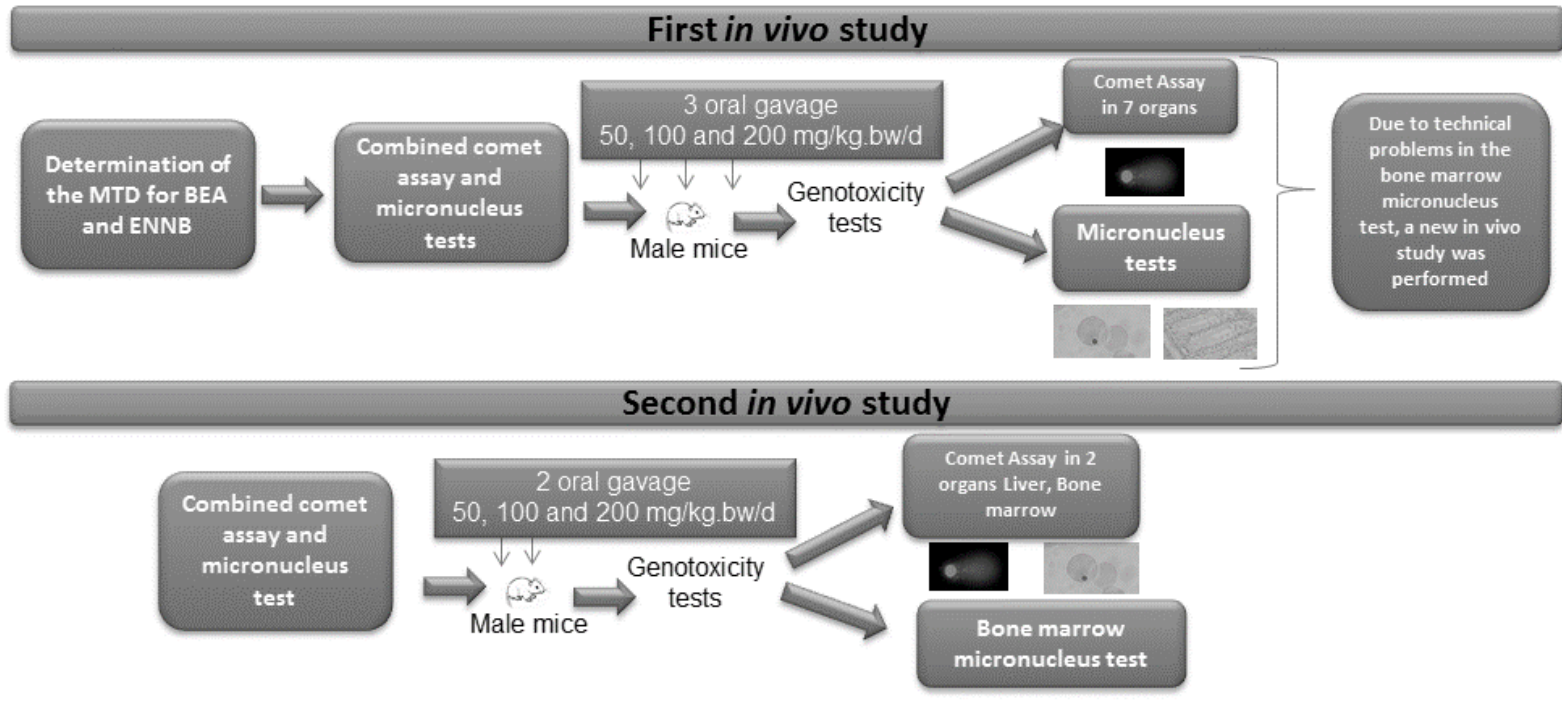

\section{In vitro genotoxicity tests}

Micronucleus test in

human TK6 cells after

a 3 hours of

treatement with and

without $\$ 9$ mix and 24

hours of treatment

w/oss

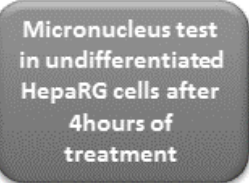

Micronucleus test

in undifferentiated

4hours of

treatment
Multiparametric

VH2AX/pH3 test in

undifferentiated

HepaRG cells

Figure 1: General overview of the task 1 on the acute in vivo genotoxicity studies and in vitro genotoxicity studies with BEA and ENNB.

\subsubsection{Solubility protocols}

\section{BEA}

A first test of solubility was performed in $5 \%$ ethanol and physiologic saline water at different concentrations of 20,10 and $5 \mathrm{mg} / \mathrm{ml}$. Due to a lack of solubility of the toxin; ethanol was replaced by DMSO as advised in the data sheet of the product. The final solution at $50 \mathrm{mg} / \mathrm{kg}$ was turbid $(10 \%$ DMSO).

Another solubility test was performed using colza oil supplemented with DMSO10\% at various concentrations of 10 and $20 \mathrm{mg} / \mathrm{ml}$. The solubility of the solutions was satisfactory. Finally, BEA was soluble in colza oil at DMSO5\%.

Therefore, final solution of gavage of BEA was performed in colza oil with DMSO5\%. A negative CTRL group has received a solution of colza oil with DMSO5\% and the positive CTRL group was dosed with MMS dissolved in physiologic saline solution at $8 \mathrm{mg} / \mathrm{ml}$.

\section{ENNB}

As recommended in the data sheet, ENNB was dissolved in DMSO. A first test of solubility at $20 \mathrm{mg} / \mathrm{ml}$ was performed with colza oil and DMSO5\%. The results showed a precipitation of the toxin. Then, the percentage of DMSO was increased up to final $10 \%$ and obtained results were acceptable.

Therefore, the final solution of ENNB was performed in colza oil at DMSO10\%. The negative CTRL group received a solution of colza oil supplemented with DMSO10\%. The positive CTRL group was dosed with MMS at $8 \mathrm{mg} / \mathrm{ml}$. 


\subsubsection{Determination of the maximum tolerated dose (MTD)}

\section{BEA}

A preliminary dose range-finding experiment was performed to determine the maximum tolerated dose (MTD) for BEA based on clinical signs of toxicity. In fact, the MTD should aim to identify the dose inducing slight toxic effects relative to the duration of the study period (clear clinical signs such as abnormal behaviour or reactions, minor body weight depression or target tissue cytotoxicity) but no death or evidence of pain (OECD TG $489^{1}$ ).

The starting BEA dose for the preliminary dose range-finding experiment was based on the only available LD50 value (100 mg/kg b.w.) found by Omura et al., 1991 in mice upon acute oral administration, as reported in EFSA Report on mycotoxins (EFSA, 2014). A first animal was dosed by gavage (volume of administration of $10 \mathrm{ml} / \mathrm{kg}$ ) with 3 oral doses of BEA at $100 \mathrm{mg} / \mathrm{kg} \mathrm{b.w}$. per day. No clinical signs of toxicity were observed during the 3-day period of treatment. A second animal was dosed with BEA at $200 \mathrm{mg} / \mathrm{kg}$ b.w. per day during 3 days and no clinical signs of toxicity were noticed. The same schedule was followed with 3 other mice at $200 \mathrm{mg} / \mathrm{kg} \mathrm{b.w}$. confirming the absence of toxicity. However, since $200 \mathrm{mg} / \mathrm{kg}$ b.w. BEA largely exceeded the highest acute human exposure estimates for BEA $(0.05 \mu \mathrm{g} / \mathrm{kg}$ b.w. per day (mean) and $0.10 \mu \mathrm{g} / \mathrm{kg}$ b.w. per day (95th percentile)) (EFSA, 2014) and considering the high cost of the mycotoxin, we decided to carry out the main acute experiment with $200 \mathrm{mg} / \mathrm{kg}$ as the highest BEA dose, even if the MTD had not be reached and this could be considered as a deviation of the agreed protocol. Therefore, the selected doses for the acute in vivo genotoxicity test were: 50, 100 and $200 \mathrm{mg} / \mathrm{kg}$ b.w. per day.

\section{ENNB}

No acute in vivo toxicity data are available for ENNB, then a preliminary range-finding study was performed to determine the MTD of ENNB.

The first animal was dosed with $200 \mathrm{mg} / \mathrm{kg}$ b.w per day at $10 \mathrm{ml} / \mathrm{kg}$ during a period of two days and no toxicity was noticed. A second animal was dosed with 2 daily doses of $200 \mathrm{mg} / \mathrm{kg} \mathrm{b.w}$. As seen previously with the first animal, no sign of toxicity was observed. As for BEA, since $200 \mathrm{mg} / \mathrm{kg}$ b.w. ENNB exceeded the highest acute human exposure estimates for the sum of ENN $(4,67 \mu \mathrm{g} / \mathrm{kg}$ b.w. per day (mean) and $10.1 \mu \mathrm{g} / \mathrm{kg}$ b.w. per day (95th percentile)) (EFSA, 2014) and considering the high cost of the mycotoxin, we decided to carry out the main acute experiment with $200 \mathrm{mg} / \mathrm{kg}$ as the highest dose. Therefore, the selected doses of ENNB were 50, 100 and $200 \mathrm{mg} / \mathrm{kg}$ b.w.

\subsubsection{Oral administration for Comet assay and Micronucleus assay}

\section{BEA}

After 5 days of acclimation period, mice were divided into 5 groups ( 5 mice/group). One group was treated at $50 \mathrm{mg} / \mathrm{kg}$ b.w. of BEA, the second group at $100 \mathrm{mg} / \mathrm{kg}$ b.w. and the third group at $200 \mathrm{mg} / \mathrm{kg}$ b.w. per day for 3 days. The negative CTRL group received a solution of colza oil supplemented with DMSO5\%. The positive CTRL group was dosed with MMS at $8 \mathrm{mg} / \mathrm{ml}$. The gavage schedule followed a daily administration at 0,24 and 45 hours $(10 \mathrm{ml} / \mathrm{kg})$. Body weights were recorded before each administration and clinical signs were monitored daily.

In the second study, two administrations of BEA by gavage in 48 hours were performed at the same dose levels and the mice were sacrificed 24 hours after the last gavage.

\section{ENNB}

In the present study, mice were divided into 5 groups ( 5 mice/group). One group was treated at $50 \mathrm{mg} / \mathrm{kg} \mathrm{b.w}$. of ENNB, the second group at $100 \mathrm{mg} / \mathrm{kg} \mathrm{b.w}$. and the third group at $200 \mathrm{mg} / \mathrm{kg}$ b.w.

\footnotetext{
${ }^{1}$ OECD, Test No. 489, 2014: In Vivo Mammalian Alkaline Comet Assay, OECD Publishing, Paris. 
per day for 2 days. The negative CTRL group received a solution of colza oil supplemented with DMSO10\%. The positive CTRL group was dosed with MMS at $8 \mathrm{mg} / \mathrm{ml}$. The gavage schedule followed a daily administration at 0 and 21 hours $(10 \mathrm{ml} / \mathrm{kg})$. Body weights were recorded before each administration and clinical signs were monitored daily.

In the second study, two administrations of ENNB by gavage in 48 hours were performed at the same dose levels.

\subsubsection{In vivo alkaline Comet assay}

\section{Sample preparation}

In the first experiment, 7 organs/tissues were collected (liver, blood, duodenum, kidney, colon, spleen and bone marrow) and only 2 organs/tissues in the second experiment (liver and bone marrow).

Each animal was anesthetized by an intraperitoneal injection of $60 \mathrm{mg} / \mathrm{kg} \mathrm{b.w}$. of sodium pentobarbital 3 hours after the last oral administration in the first study and 24 hours after the second administration in the second experiment. After blood collection in heparinized tube, a perfusion through the heart removing the blood from the organs was established with $30 \mathrm{ml}$ of HBSS medium at $3 \mathrm{ml} / \mathrm{min}$. Colon, spleen, kidney, liver and duodenum were removed and kept on ice and rinsed with HBSS medium supplemented with DMSO10\%. Liver and kidney were cut into small pieces. Cells were mechanically isolated (Medimachine $\AA$, Dako, France) and filtered. Spleen cells were isolated by flushing with pipette. Bone marrow cells were collected from isolated femur using $1 \mathrm{ml}$ of fetal calf serum. Duodenum and colon were rinsed with HBSS medium with DMSO10\%, cut longitudinally and scrapped with a coverslip. Cell suspension was filtered twice. Cell pellets of colon, duodenum, liver, kidney and spleen were obtained after centrifugation step at $800 \mathrm{~g}$ for 4 minutes.

\section{Electrophoretic migration of DNA}

Blood sample $(20 \mu \mathrm{l})$, bone marrow $(20 \mu \mathrm{l})$ and cell pellets were mixed with $0.8 \%$ low melting-point (LMP) agarose $(300 \mu \mathrm{l})$, and $65 \mu \mathrm{l}$ of cell suspension was seeded on slides pre-coated with $1 \%$ normal melting agarose. Three slides/organ were set up; except for blood (2 slides). Cell lysis was obtained with a lysis solution (2.5 M NaCl, $0.1 \mathrm{M}$ EDTA, $10 \mathrm{mM}$ Tris $-\mathrm{HCl} \mathrm{pH} \mathrm{10}$, extemporarily added with DMSO $10 \%$ and $1 \%$ Triton $\mathrm{X}-100)$ for 1 hour at $4^{\circ} \mathrm{C}$. DNA was allowed to unwind 20 minutes in fresh electrophoresis buffer (0.3 M NaOH, $1 \mathrm{mM}$ EDTA, pH13) before electrophoretic migration (24 minutes, $0.35 \mathrm{~V} / \mathrm{cm}, 300 \mathrm{~mA}$ in the first experiment and 24 minutes, $0.7 \mathrm{~V} / \mathrm{cm}, 300 \mathrm{~mA}$ in the second experiment). Slides were bathed two times for 5 minutes into a neutralizing solution $(0.4 \mathrm{M} \mathrm{Tris}-\mathrm{HCl}$, $\mathrm{pH} 7.5$ ) then fixed with ethanol $95 \%$ for 5 minutes.

\section{DNA staining and slides scoring}

Coded slides were stained with propidium iodide $(20 \mu \mathrm{g} / \mathrm{ml}$ in Phosphate Buffered Saline, PBS) and analyzed by the Comet Assay IV software (Perceptive Instruments, Haverhill, UK). Fifty cells per slide (on 3 slides per organ per animal) were analyzed with the exception of blood for which 75 cells per slide (on 2 slides per animal) were analyzed. DNA damage was expressed as percentage of DNA in the tail (\% Tail Intensity, TI). The frequency of hedgehogs was scored for each slide.

According to Comet assay OECD TG $489^{1}$, the tested substance is considered able to induce DNA strand breakage in the tissues when:

- at least one of the test doses has set up a statistically significant increase compared with the negative CTRL

- $\quad$ the increase is dose-related monitored with an appropriate trend test

\footnotetext{
${ }^{1}$ OECD, Test No. 489, 2014: In Vivo Mammalian Alkaline Comet Assay, OECD Publishing, Paris. 
- any of the results are outside the distribution of the historical negative CTRL data for a given species, vehicle, route, tissue and number of administrations.

\subsubsection{Micronucleus assay}

\section{Bone marrow}

Bone marrow smears were prepared from femurs and foetal bovine serum (FBS). Slides were stained with May Grunwald Giemsa technique. Two slides per animal were prepared and the proportion of immature among total erythrocytes (PCE/NCE) was determined from the microscopic examination for each animal by counting a total of at least 500 erythrocytes. At least 2000 immature erythrocytes (PCE) were scored per animal for the incidence of micronuclei.

\section{Colon crypts}

Colon was cut longitudinally, washed, rolled, fixed in $4 \%$ formalin and embedded in paraffin. Colon was cut with $5 \mu \mathrm{m}$ sections, deparaffinised and alcohol dehydrated 5 min in 100, 90 and $70 \%$ ethanol successively. Slides were stained with Feulgen (1h at room temperature) and $2 \mathrm{~h}$ with Schiff's reagent. The sections were counterstained with Fast Green. Intact colon crypts were chosen and 1000 epithelial cells were scored focus on micronucleated cells count. A micronucleated cell was defined as a cell with a normal nucleus adjacent to one or two micronuclei in the cytoplasm with a diameter never exceeding one-third of the main nucleus. Apoptosis was assessed on the basis of morphological characteristics, such as cell shrinkage, chromatin condensation or cellular fragmentation. Mitotic cells were identified too.

\subsubsection{Immunohistochemistry protocol for $\mathrm{yH} 2 \mathrm{AX}$ detection in formalin-fixed paraffin-embedded kidney sections.}

The detection of DNA damage in kidney sections of mice exposed to ENNB was performed by using the detection of the phosphorylation of $\mathrm{H} 2 \mathrm{AX}$ at the serine 139 in order to confirm the result in the Comet assay observed at the lower dose.

Formalin-fixed paraffin-embedded tissue sections were deparaffinised and re-hydrated through graded alcohols. Rabbit monoclonal anti-yH2AX antibody form Cell signaling (ref:2577 at 1:50) was used followed by the DABMap kit for Streptavidin-biotin peroxydase detection by using the Discovery XT platform (Roche). Slides were scored by using NIS-Elements (Nikon) and $\mathrm{yH} 2 \mathrm{AX}$ positive cells $/ \mathrm{mm}^{2}$ was determined in 5 different locations in each slide.

\subsubsection{Histopathological analysis in the liver}

In order to know if BEA induced systemic toxicity effect in mice after 3 oral gavages, we performed histopathological analysis of liver slides. Briefly, paraffin-embedded liver slides were stained with Haematoxylin and Eosin prior to slides being read. Histological processing and examination was conducted in accordance with the test site standard operating procedures. Microscopic examination was performed as follows: steatosis, inflammation, mitosis, clarification and necrosis were reported as present and assigned a severity grade. In the latter case, one of the following five grade was used: minimal, slight, moderate, marked or severe.

\subsection{In vitro genotoxicity evaluation of BEA and ENNB}

A series of in vitro tests was performed in order to determine the genotoxic mode of action of toxins. The MN test based on the OECD TG N $487^{1}$ was performed in human TK6 cells and in human proliferative HepaRG cells. The in vitro MN test is a genotoxicity test for the detection of micronuclei in the cytoplasm of interphase cells. Micronuclei can originate from acentric chromosome fragments or whole chromosomes that are unable to migrate to the poles during the anaphase stage of cell

\footnotetext{
${ }^{1}$ OECD, Test No. 487, 2010: In Vitro Mammalian Cell Micronucleus Test, OECD Publishing, Paris. 
division. Therefore, the in vitro $\mathrm{MN}$ test is a method that provides a comprehensive basis for investigating chromosome damaging potential in vitro because both aneugens and clastogens can be detected (OECD N 487 ${ }^{1}$ ).

More, we also performed multiparametric $\mathrm{yH} 2 \mathrm{AX}$ and $\mathrm{pH} 3$ test in proliferative human HepaRG cells in order to determine the clastogenic or the aneugenic potential of BEA and ENNB. The phosphorylation of histone $\mathrm{H} 2 \mathrm{AX}$ in the serine 139 (named $\mathrm{yH} 2 \mathrm{AX}$ ) is induced in case of DNA damage (Rogakou et al., 1998) and the antibody against $\mathrm{yH} 2 \mathrm{AX}$ is used to visualize double-strand breaks of DNA. The phosphorylation of histone $\mathrm{H} 3$ at serine 10 (named $\mathrm{pH} 3$ ) is specifically induced at the mitosis phase, the antibody against $\mathrm{pH} 3$ specifically stains mitotic cells allowing the distinction of aneugenic compounds that increase the number of mitotic cells from clastogenic compounds that have no effect on this parameter (Bryce et al., 2017; Khoury et al., 2016).

\subsubsection{In vitro Micronucleus assay in undifferentiated human HepaRG cells}

Proliferative HepaRG cells were seeded at a density a density of $2.6 \times 10^{4}$ cells $/ \mathrm{cm}^{2}$ in a 2 chambers Lab-Tek slide system ${ }^{\circledR}$. Twenty-four hours after seeding, the cells were exposed to increasing concentrations of toxins for $4 \mathrm{hrs}$. MMS at $60 \mu \mathrm{g} / \mathrm{ml}$ was used as positive CTRL and negative CTRL was DMSO0.2\%. At the end of the treatment, the cells were incubated for $24 \mathrm{~h}$ in fresh medium containing $10 \% \mathrm{FBS}$ and $3 \mu \mathrm{g} / \mathrm{ml}$ of cytochalasin-B (Cyt-B). At the end of Cyt-B treatment, the cells were washed twice with PBS and allowed to recover for $1.5 \mathrm{~h}$ in a $10 \%$ FBS supplemented-fresh medium prior to fixation in methanol/acetic acid $(9 / 1, v / v)$ for $5 \mathrm{~min}$. Slides were stained with acridine orange $(67 \mu \mathrm{g} / \mathrm{ml}$ in PBS). All slides were scored blindly. The criteria used for identifying micronuclei were those recommended by the HUMN project (Fenech et al., 2003). Micronuclei were scored in 1000 binucleated cells per assay. Mononucleated and polynucleated cells were also counted. For cytotoxicity assessment, the cytostasis was determined, where cytostasis $=100-\mathrm{RI}$ (Replication Index $) . R I=[($ number of binucleated cells $+2 \times$ polynucleated cells $) /($ Total cell number $)] \times 100$.

\subsubsection{In vitro Micronucleus assay in human TK6 cells}

The human TK6 lymphoblastoid cell line was maintained as suspension culture in RPMI 1640 medium with Glutamax ${ }^{\mathrm{TM}}$ and supplemented with $10 \% \mathrm{FBS}, 100 \mathrm{IU} / \mathrm{ml}$ penicillin and $100 \mu \mathrm{g} / \mathrm{ml}$ streptomycin. The average doubling time of the TK6 cells was 10-12 h. TK6 cells $\left(2 \times 10^{5} \mathrm{cells} / \mathrm{ml}\right)$ were treated with BEA or ENNB in $5 \%$ FBS culture medium for 3 hours with and without rat ( $\beta$-naphtoflavone and phenobarbital-induced) liver S9 fractions mix and $24 \mathrm{~h}$ without S9.

Liver S9 fractions were purchased from Moltox (Germany). The S9-mix included 10\% S9 fraction, 8 $\mathrm{mM} \mathrm{MgCl} 2,32 \mathrm{mM} \mathrm{KCl}, 5 \mathrm{mM}$ glucose-6-phosphate, $4 \mathrm{mM}$ NADP and $100 \mathrm{mM}$ sodium phosphate ( $\mathrm{pH}$ 7.4). For short time exposure $(3 \mathrm{~h})$, cells were treated with toxins in the absence or in the presence of S9 ( $2 \%$ S9 final concentration). Then the treatment medium was replaced by $10 \%$ FBS fresh medium and cells were collected $21 \mathrm{~h}$ later. For the long time exposure, TK6 cells were treated with toxins for $24 \mathrm{~h}$. Benzo[a]pyrene $(\mathrm{B}[\mathrm{a}] \mathrm{P})(8 \mu \mathrm{M})$ and MMS $(45 \mu \mathrm{M})$ were chosen as positive CTRLs with and without S9 mix, respectively. Cells were collected by centrifugation (136 g for $5 \mathrm{~min}$ ) and counted for cytotoxicity evaluation: relative increase in cell count (RICC), relative population doubling (RPD) and relative cell counts (RCC) were calculated according to the OECD TG $487^{1}$. For MN scoring, the cell pellets were treated for 4 min with a hypotonic solution (RPMI medium: distilled water $=1: 1(\mathrm{v} / \mathrm{v})$ ) prior to fixation (methanol: acetic acid $=3: 1(\mathrm{v} / \mathrm{v})$ ) for $10 \mathrm{~min}$ at room temperature. Cells were centrifuged again, resuspended in the fixative solution, spread on glass slides and stained with acridine orange $(67 \mu \mathrm{g} / \mathrm{ml}$ in PBS). Micronuclei were scored in 2000 mononucleated cells in duplicate culture under a fluorescence microscope (Leica DMR, Germany).

\footnotetext{
${ }^{1}$ OECD, Test No. 487, 2010: In Vitro Mammalian Cell Micronucleus Test, OECD Publishing, Paris. 


\subsection{3. $\quad \mathrm{YH} 2 \mathrm{AX} / \mathrm{pH}$ staining in undifferentiated HepaRG cells}

Proliferant HepaRG cells were seeded, $24 \mathrm{hr}$ prior to incubation with the toxins, at a density of 50,000 cells/ $\mathrm{cm}^{2}$ 96-well-plates (Corning). After incubation with toxins, cells were fixed with $4 \%$ paraformaldehyde in PBS (Thermo Fisher Scientific) and permeabilized with $0.2 \%$ Triton in PBS. Plates were then incubated in blocking solution BSA $1 \%$, Tween $200.05 \%$ in PBS (Thermo Fisher Scientific) for $30 \mathrm{~min}$ prior to the addition of antibodies solutions. Primary antibodies (mouse monoclonal anti yH2AX ser139 ab26350 from Abcam and polyclonal rabbit anti-Histone H3 phospho S10, ab5176 from Abcam) at 1:1000, were incubated overnight at $4^{\circ} \mathrm{C}$. After washing in PBS Tween $200.05 \%$, secondary antibodies were used at $1: 1000$ and incubated $45 \mathrm{~min}$ at room temperature. Secondary antibodies were purchased from Abcam (anti-Rabbit IgG DyLight $\AA 488$ ab96891, and anti-Mouse IgG H\&L DyLight ${ }^{\circledR} 550$ ab96876). DAPI staining was used as a nuclear counterstain for automated cell identification by high content screening.

Plates were scanned using the Thermo Scientific ArrayScan VTI HCS Reader (Thermo Fisher Scientific) and analyzed using the Compartmental Analysis BioApplication (Thermo Fisher Scientific). For each well, 9 fields (20X magnification) were scanned and analyzed for nuclear and immunostaining based parameters. Cell survival was determined by cell counting from DAPI staining and expressed as a percentage of cells compared to CTRL condition. $\mathrm{yH} 2 \mathrm{AX}$ and $\mathrm{pH} 3$-ser10 were quantified in cell nucleus and expressed as a fold of activation compared to CTRL.

\subsection{Repeated dose oral toxicity study (OECD TG $407^{1}$ ) and} Reproductive/ Developmental Toxicity Screening Test (OECD TG $422^{2}$ ) in rodents

\subsubsection{Selection of dose levels}

For both the mycotoxins, the LD50, reported in EFSA opinion (EFSA, 2014), is the only reference value available in literature and it has been considered as starting point to calculate the dose levels to be administered to mice in the repeated dose study. The high dose level was obtained by dividing the LD50 for a 10 factor for BEA and for a 20 factor for ENNB, considering the long exposure period, in order to avoid overt toxicity. Mid and low doses were calculated according to the OECD TG $407^{1}$, taking also into account that there may be differences in sensitivity between pregnant and nonpregnant animals. For ENNB, the low dose level is comparable with the rough estimate for a Lowest Observed Adverse Effect Level (LOAEL) for a short-term exposure established by EFSA - 0.09 to 0.17 $\mathrm{mg} / \mathrm{kg}$ b.w. - considering the therapeutic treatment with Locabiotal ${ }^{\circ}$ (a mixture of ENNs) (Table 1).

Table 1: Dose selection of BEA and ENNB for the repeated dose oral toxicity study and screening of reproductive and developmental endpoints

\begin{tabular}{|c|c|c|c|c|}
\hline Mycotoxin & LD50 & $\begin{array}{l}\text { High dose } \\
\text { (H) }\end{array}$ & $\begin{array}{c}\text { Middle } \\
\text { dose (M) }\end{array}$ & $\begin{array}{l}\text { Low dose } \\
\text { (L) }\end{array}$ \\
\hline BEA & $\begin{array}{l}100 \\
\mathrm{mg} / \mathrm{kg} \text { b.w. }\end{array}$ & $\begin{array}{l}10 \mathrm{mg} / \mathrm{kg} \text { b.w. } \\
(=1 / 10 \text { LD50) }\end{array}$ & $\begin{array}{l}1 \mathrm{mg} / \mathrm{kg} \\
\text { b.w. }\end{array}$ & $\begin{array}{l}0.1 \mathrm{mg} / \mathrm{kg} \\
\text { b.w. }\end{array}$ \\
\hline ENNB & $\begin{array}{l}350 \\
\mathrm{mg} / \mathrm{kg} \text { b.w. }\end{array}$ & $\begin{array}{l}18 \mathrm{mg} / \mathrm{kg} \text { b.w. } \\
(=1 / 20 \text { LD50) }\end{array}$ & $\begin{array}{l}1.8 \mathrm{mg} / \mathrm{kg} \\
\text { b.w. }\end{array}$ & $\begin{array}{l}0.18 \mathrm{mg} / \mathrm{kg} \\
\text { b.w. }\end{array}$ \\
\hline
\end{tabular}

\footnotetext{
${ }^{1}$ OECD, Test No. 407, 2008: Repeated Dose 28-day Oral Toxicity Study in Rodents, OECD Publishing, Paris.

${ }^{2}$ OECD, Test No. 422, 1996: Combined Repeated Dose Toxicity Study with the Reproduction/Developmental Toxicity Screening Test, OECD Publishing, Paris.
} 


\subsubsection{Solubility protocol}

\section{BEA}

Stock solution: BEA powder was dissolved in DMSO. $50 \mathrm{mg}$ of powdered BEA was added to $750 \mu \mathrm{l}$ of DMSO. To ensure the complete solubilisation, the mixture was vortexed for 2 hours. When the final solution appeared perfectly clear and without visible mycotoxin residuals, it was stored at $-20^{\circ} \mathrm{C}$ until use.

Gavage solution: for each dose level, final concentration of BEA in olive oil + DMSO1.5\% was obtained by proportionally diluting the stock solution. The gavage solution was mixed $\mathrm{O} / \mathrm{N}$ and stored at $+4^{\circ} \mathrm{C}$ until the use.

\section{ENNB}

Stock solution: ENNB powder was dissolved in DMSO. $100 \mathrm{mg}$ of powdered ENN B was added to 3.5 $\mathrm{ml}$ of DMSO. To obtain a complete solubilisation, the mixture was vortexed $\mathrm{O} / \mathrm{N}$. When the final solution appeared perfectly clear and without visible mycotoxin residuals, it was stored at $-20^{\circ} \mathrm{C}$ until use.

Gavage solution: for each dose level, final concentration of ENNB in olive oil+ DMSO6\% was obtained by proportionally diluting the stock solution. The gavage solution was mixed $\mathrm{O} / \mathrm{N}$ and stored at $+4^{\circ}$ $C$ until use.

\subsubsection{Animals and treatment for BEA and ENNB studies}

The study was conducted according to the European Community Council Directive 2010/63/UE ${ }^{1}$ the Italian Law 4 marzo 2014 n. $26^{2}$ and the OECD Principles on GLP.

The general overview of the study design is shown in Figure 2.

\footnotetext{
${ }^{1}$ DIRECTIVE 2010/63/EU OF THE EUROPEAN PARLIAMENT AND OF THE COUNCIL of 22 September 2010 on the protection of animals used for scientific purposes http://ec.europa.eu/environment/chemicals/lab_animals/legislation_en.htm ${ }^{2}$ DECRETO LEGISLATIVO 4 marzo 2014, n. 26. Attuazione della direttiva 2010/63/UE sulla protezione degli animali utilizzati a fini scientifici. (14G00036). (GU n.61 del 14-3-2014)
} 


\section{Task 2-3: Repeated dose oral toxicity of BEA and ENNB - experimental schedule}

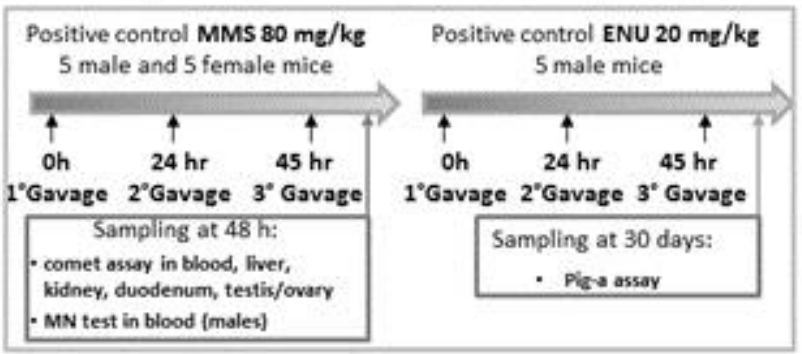

$10 \mathrm{CD}-1$ male-female-dam mice/eroup

BEA experiment

Treatments: olive oit, $0.1,1,10 \mathrm{mg} / \mathrm{kg}$ BEA

ENNB experiment

Treatments: olive oiltoMsO $\%, 0.18,1.8,18 \mathrm{~m} / \mathrm{kg}$ ENNB

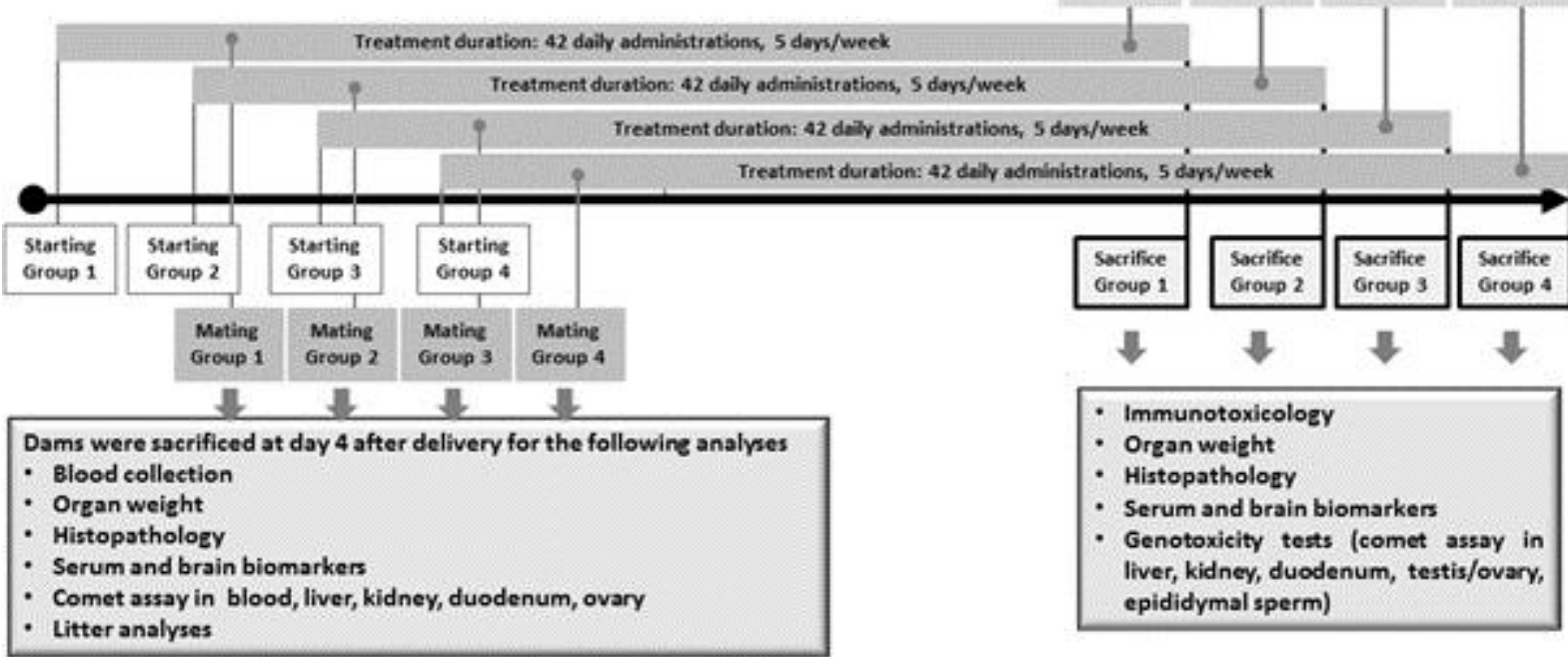

Figure 2: General overview of the tasks 2-3: repeated dose oral toxicity studies and screening of reproductive and developmental toxicity with BEA and ENNB. BEA study started on September 19, 2016. ENNB study started on January 13, 2017. BEA group 1: CTRL (0 olive oil); group 2: $0.1 \mathrm{mg} / \mathrm{kg}$ b.w. per day; group 3: $1 \mathrm{mg} / \mathrm{kg}$ b.w. per day; group 4: 10 $\mathrm{mg} / \mathrm{kg}$ b.w. per day. ENNB group 1: $18 \mathrm{mg} / \mathrm{kg}$ b.w. per day; group 2: $1.8 \mathrm{mg} / \mathrm{kg}$ b.w. per day; group 3: $0.18 \mathrm{mg} / \mathrm{kg}$ b.w. per day; group 4: CTRL (0 - olive oil+DMSO6\%)

Male (44, seven weeks old) and female (84, six weeks old) CD-1 mice, average b.w. $30 \mathrm{~g}$, were obtained from Charles River Laboratories (Italy) and kept under standard laboratory conditions (22 \pm $0.5{ }^{\circ} \mathrm{C}, 50-60 \%$ relative humidity, $12 \mathrm{~h}$ dark-light alternation with 12-14 air changes/h). Starting from the acclimatizing period, mice were fed with the rodent diet CA.170481 AIN-76A Purified Diet (Rats/Mice) (Harlan Teklad, Italy) evaluated among the available formulas in order to supply as lesser amount of BEA and ENNB as possible. Water was available ad libitum. After 5 days of acclimation, mice were divided as summarized below (Table 2 and 3 ). 
Table 2: Scheme of BEA treatment groups. Male and female groups included $10 \%$ additional animals to take into account possible deaths

\begin{tabular}{|c|c|c|c|c|c|}
\hline & & CTRL & BEA0.1 & BEA1 & BEA10 \\
\hline BEA dose level & & 0 mg/kg b.w. & $0.1 \mathrm{mg} / \mathrm{kg}$ b.w. & $1 \mathrm{mg} / \mathrm{kg}$ b.w. & $10 \mathrm{mg} / \mathrm{kg}$ b.w. \\
\hline Vehicle & & olive oil & $\begin{array}{c}\text { olive oil + } \\
\text { DMSO1.5\% }\end{array}$ & $\begin{array}{c}\text { olive oil + } \\
\text { DMSO1.5\% }\end{array}$ & $\begin{array}{c}\text { olive oil + } \\
\text { DMSO1.5\% }\end{array}$ \\
\hline Gavage volume & & $1 \mathrm{ml} / 100 \mathrm{~g} \mathrm{b.w}$. & $1 \mathrm{ml} / 100 \mathrm{~g} \mathrm{b.w.}$ & $1 \mathrm{ml} / 100 \mathrm{~g}$ b.w. & $1 \mathrm{ml} / 100 \mathrm{~g} \mathrm{~b} . \mathrm{w}$. \\
\hline N. of mice/group & male mice & 11 & 11 & $11^{* *}$ & 11 \\
\hline & female mice & $11^{* *}$ & $11^{*}$ & $11^{*}$ & 11 \\
\hline & dams & 10 & 10 & 10 & 10 \\
\hline
\end{tabular}

**2 mice died due to gavage procedure; *1 mouse died due to gavage procedure

Table 3: Scheme of ENNB treatment groups. Male and female groups included $10 \%$ additional animals to take into account possible deaths

\begin{tabular}{|c|c|c|c|c|c|}
\hline & & CTRL & ENNB0.18 & ENNB1.8 & ENNB18 \\
\hline ENNB dose level & & $0 \mathrm{mg} / \mathrm{kg}$ b.w. & $\begin{array}{c}0.18 \mathrm{mg} / \mathrm{kg} \\
\text { b.w. }\end{array}$ & $1.8 \mathrm{mg} / \mathrm{kg}$ b.w. & 18 mg/kg b.w. \\
\hline Vehicle & & $\begin{array}{l}\text { olive oil + } \\
\text { DMSO6\% }\end{array}$ & $\begin{array}{l}\text { olive oil + } \\
\text { DMSO6\% }\end{array}$ & $\begin{array}{l}\text { olive oil + } \\
\text { DMSO6\% }\end{array}$ & $\begin{array}{l}\text { olive oil + } \\
\text { DMSO6\% }\end{array}$ \\
\hline Gavage volume & & $1 \mathrm{ml} / 100 \mathrm{~g} \mathrm{b.w}$. & $1 \mathrm{ml} / 100 \mathrm{~g}$ b.w. & $1 \mathrm{ml} / 100 \mathrm{~g}$ b.w. & $1 \mathrm{ml} / 100 \mathrm{~g}$ b.w. \\
\hline N. of mice/group & male mice & 11 & $11^{*}$ & 11 & $11^{* *}$ \\
\hline & female mice & 11 & $11^{*}$ & 11 & 11 \\
\hline & dams & 10 & 10 & 10 & 10 \\
\hline
\end{tabular}

**4 mice died due to gavage procedure; *1 mouse died due to gavage procedure

\section{Repeated dose oral toxicity study with BEA and ENNB}

The study protocol was the same for both BEA and ENNB.

Adult male and female CD-1 mice (10 male mice/group and 10 female mice/group) were used. After the 5-day acclimatizing period, male and female mice were orally administered with the dose levels of BEA and ENNB (see Table 2 and 3, respectively), by gavage. All the mice were treated daily for 42 days with a treatment schedule of 5 treatments/week (NanoReg, 2017 ; NTP, 19932). Animals were checked daily during the treatment period for their health status. Suffering and/or moribund mice were humanely sacrificed. Body weight gain and food consumption were recorded three times a week during the treatment period. A few days before sacrifice, $200 \mu$ blood were harvested from the periorbital venous sinus of 5 mice/group for repeated dose genotoxicity endpoints.

After 42 treatment days, virgin females and sires from treated and CTRL groups were anaesthetized with a gaseous solution of isofluorane, blood samples were collected by intracardiac puncture for biomarkers determination ( 5 mice per sex/group) and immunological parameters (5 mice per sex/group). Subsequently, animals were sacrificed by $\mathrm{CO}_{2}$ asphyxiation.

\section{Positive control for Comet assay and Micronucleus test}

CD-1 male and female mice were treated orally by gavage. The delivered volume was $10 \mathrm{ml} / \mathrm{kg}$ b.w. Three doses of $80 \mathrm{mg} / \mathrm{kg}$ b.w. MMS at 0,24 , and $45 \mathrm{~h}$ were administered and mice were sacrificed 48 $\mathrm{h}$ after the first administration. The positive CTRL was constituted by 5 males and 5 females for the Comet assay and by 5 males for the MN test. These treatment groups were considered positive CTRL for both BEA and ENNB experiments. In agreement with the 3R principle, this strategy was used to reduce the number of animals.

\footnotetext{
${ }^{1}$ NANoREG - NP-LA-2013-310584. Final Report (part 1) November 2016 / updated 2017/02/21.

${ }^{2}$ National Toxicology Program (NTP). Natl Toxicol Program Tech Rep Ser. 1993 Apr;414:1-284
} 


\section{Positive control for Pig-a assay}

Five CD-1 male mice treated orally by gavage with three daily-doses of $20 \mathrm{mg} / \mathrm{kg} \mathrm{b.w.} \mathrm{ENU} \mathrm{delivered}$ in a volume $10 \mathrm{ml} / \mathrm{kg}$ b.w. and sacrificed 30 days after treatment, constituted the positive CTRL for the Pig-a assay. These treatment groups were considered positive CTRL for both BEA and ENNB experiments. In agreement with the $3 \mathrm{R}$ principle, this strategy was used to reduce the number of animals.

\section{Reproduction/Developmental Toxicity Screening test with BEA and ENNB}

The study protocol was the same for both BEA and ENNB.

After two weeks of treatment, male mice from the repeated dose oral toxicity study (10/group) were mated with virgin female mice (10/group) for screening reproductive and developmental endpoints, on the basis of the OECD TG $422^{1}$.

One male and one female mice from treated and CTRL groups were housed (1:1 mating) in one cage until pregnancy occurred or two weeks elapsed. Each morning the females were examined for the presence of sperm or a vaginal plug. Day 0 of pregnancy was defined as the day of positive vaginal plug. Dams were treated throughout pregnancy including the day 4 post-partum before sacrifice, with a treatment schedule of 5 treatments/week (NanoReg, 2017²; NTP, 1993³). Each litter was examined as soon as possible after delivery to establish the number and sex of pups, stillbirths, live births, and the presence of gross abnormalities. Dams and pups were killed by $\mathrm{CO}_{2}$ asphyxiation at day 4 postpartum and carefully examined externally for gross abnormalities. Before sacrifice, dams were anaesthetized with a gaseous solution of isofluorane, blood samples were collected by intracardiac puncture for biomarker determination. After the mating period, sires continued the treatment regimen until the end of the experiment in order to enable the detection of the majority of effects on male fertility and spermatogenesis, and sacrificed.

\subsubsection{General toxicity}

The endpoints are the same for both BEA and ENNB.

\section{Male and female mice}

After the sacrifice, necropsy and gross pathology were performed on all the CTRL and treated mice. The following organs were excised and weighed: liver, spleen, kidneys, adrenals, brain, thyroid (weighed after fixation), mesenteric lymph nodes (MLN), thymus, heart, uterus, testis, epididymis and ovary. The duodenum (both sexes) and mammary glands (females only) were not weighed following excision, due to the difficulty in the standardization of the tissue portion trimmed at sacrifice.

\section{Dams}

After the sacrifice, necropsy and gross pathology were performed on all the CTRL and treated dams. The following organs were excised and weighed: liver, spleen, kidneys, adrenals, brain, thyroid (weighted after fixation), lymph nodes, thymus, heart, and ovary. The duodenum was not weighed following excision due to the difficulty in the standardization of the tissue portion trimmed at sacrifice. Uterus was not weighed (OECD TG $422^{1}$ ) and observed to check the number of implants and resorptions.

\section{Pups/Litters}

Each litter was examined as soon as possible after delivery to establish the number and sex of pups, stillbirths, live births, runts (pups that are significantly smaller than corresponding CTRL pups), and

\footnotetext{
${ }^{1}$ OECD, Test No. 422, 1996: Combined Repeated Dose Toxicity Study with the Reproduction/Developmental Toxicity Screening Test, OECD Publishing, Paris.

${ }^{2}$ NANOREG - NP-LA-2013-310584. Final Report (part 1) November 2016 / updated 2017/02/21.

${ }^{3}$ National Toxicology Program (NTP). Natl Toxicol Program Tech Rep Ser. 1993 Apr;414:1-284
} 
the presence of gross abnormalities by direct observation. Sex of pups was checked by observing the ano-genital distance which is in male mice approximately twice as long as that in female mice. Sex was checked again at post natal day (PND) 4 before sacrifice. At PND 4, pups were sexed, weighted and sacrificed by $\mathrm{CO}_{2}$ asphyxiation.

\subsubsection{Histopathological analysis}

After sampling, the following organs were fixed in $10 \%$ buffered formalin: liver, spleen, kidneys, duodenum, adrenals, brain, thyroid, lymph nodes, thymus, heart, mammary glands (females only), uterus, epididymis and ovary. Testes were fixed in Bouin's solution. Sperm count was performed on cauda of epididymis of 5 adult male mice (the results are reported in Appendix D and $\mathrm{E}$ ). Tissues were embedded in paraffin, cut into $5 \mu \mathrm{m}$ sections and stained with haematoxylin and eosin for light microscopy (Nikon Microphot FX) with different lenses. The histopathological alterations were described, wherever possible, according to distribution, severity and morphologic character. The scoring of the lesions was done semi quantitatively, using a 5-point grading scale (0 to 4), taking into consideration the severity of the changes based on the criteria explained by Shackelford et al, 2002, and summarized as follows:

- Grade 0: No change

- Grade $1(+1)$ : Minimal. This corresponds to a histologic change that may be barely noticeable to changes considered so minor, small, or infrequent as to warrant no more than the least assignable grade $(0-10 \%)$. For focal, multifocal or diffusely distributed lesions, this grade is used for processes where $<10 \%$ of the tissue is involved. For hyperplastic/hypoplastic/atrophic lesions, this grade is used when the affected structure or tissue has undergone $<10 \%$ increase or decrease in volume.

- Grade $2(+2)$ : Mild. This corresponds to a histologic change that is a noticeable but not prominent feature of the tissue. For focal, multifocal or diffusely distributed lesions, this grade is used for processes where $11-20 \%$ of the tissue is involved. For hyperplastic/hypoplastic/atrophic lesions, this grade is used when the affected structure or tissue has undergone between $11 \%$ and $20 \%$ increase or decrease in volume.

- Grade $3(+3)$ : Moderate. This corresponds to a histologic change that is a prominent feature of the tissue. For focal, multifocal or diffusely distributed lesions, this grade is used for processes where $21-40 \%$ of the tissue section is involved. For hyperplastic/hypoplastic/atrophic lesions, this grade is used when the affected structure or tissue has undergone between $21 \%$ and $40 \%$ increase or decrease in volume.

- Grade $4(+4)$ : Marked. This corresponds to a histologic change that is an overwhelming feature of the tissue. For focal, multifocal or diffusely distributed lesions, this grade is used for processes where $41-100 \%$ of the tissue section is involved. For hyperplastic/hypoplastic/atrophic lesions, this grade is used when the affected structure or tissue has undergone between $41 \%$ and $100 \%$ increase or decrease in volume.

Qualitative analysis of testis was performed as follow: at least 50 different tubules were observed and the scoring of lesions was done quantitatively, using percentages.

Quantitative histomorphometrical analyses were performed on thyroid, spleen, thymus, adrenals, uterus, ovary and testis of CTRL and treated mice according to Maranghi et al. (2009). Briefly, slides of selected tissues were examined by means of an image analysis system (Nis-Elements D) applied to an optical microscope (Nikon Microphot FX).

- Thyroid: using a 10x objective, follicular density, as ratio between number of follicles and a pre-determined thyroid area was measured.

- Spleen: in entire central splenic section, red pulp and white pulp areas were measured using a $2 x$ objective; ratio between red and white pulp areas was calculated.

- Thymus: cortex and medulla areas were measured using a $2 x$ objective; ratio between cortex and medulla areas was calculated. 
- Adrenal: cortex and medulla areas were measured using a $2 x$ objective; ratio between cortex and medulla areas was calculated in whole transversal section.

- Uterus: using a 10x objective, a cross-section was taken of the right uterine horn, $1 \mathrm{~cm}$ above the uterine bifurcation. The ratio between the area of endometrium and myometrium was measured as relative percentage of both uterine tissue components.

- Ovary: the classification of the different follicles was performed according to Fortune (2003). Using one of the largest sections in a central position of the ovary, primary and secondary follicles, corpora lutea, Graaf follicles, atretic follicles were counted in the whole ovarian section ( $4 \mathrm{x}$ lens). Ovaries area was measured and follicular density was calculated as ratio by total number of follicules and areas.

- Testis: Using a 20x lens, both the tubular diameters and the relative area of the seminiferus tubules and lumen were measured in 20 randomly selected tubules.

\subsubsection{Repeated dose study genotoxicity endpoints}

For the analysis of the in vivo genotoxicity endpoints within the repeated dose study, blood, liver, kidney, duodenum, ovary, testis and epididymis were processed to be analyzed by Comet assay. Blood was collected to be analyzed by Comet assay, Pig-a assay and MN assay. Blood was collected (2-4 h after the daily treatment) a few days before sacrifice; tissues were sampled $2-4 \mathrm{~h}$ after the last treatment. For Comet assay, the sampling time is a critical variable because it is determined by the period needed for the test chemicals to reach maximum concentration in the target tissue and for DNA strand breaks to be induced but before those breaks are removed, repaired or lead to cell death (OECD TG $489^{1}$ ). Taking into account this consideration and the time schedule of the treatments (5 days/week with the exclusion of Saturdays and Sundays) tissues and organs were never collected on Mondays in order to sample cells not later than 1 day after the penultimate dose.

\section{BEA}

Each dose group included 6 animals with the aim to collect data on a minimum of 5 . Alkaline Comet assay was performed on blood, liver, kidney, duodenum and testis/ovary cells of 5 males, 5 virgin females and 5 dams per experimental group [CTRL (olive oil), BEA $0.1,1$ or $10 \mathrm{mg} / \mathrm{kg} \mathrm{b.w.].} \mathrm{Sperm}$ from cauda epididymis were sampled, counted and stored at $-80{ }^{\circ} \mathrm{C}$ for successive neutral Comet assay. Pig-a assay and MN test were performed on blood of 5 males per experimental group.

\section{ENNB}

Each dose group included 6 animals with the aim to collect data on a minimum of 5 . Alkaline Comet assay was performed on blood, liver, kidney, duodenum and testis/ovary cells of 5 males, 5 virgin females and 5 dams per experimental group [CTRL (olive oil+DMSO6\%), ENNB $0.18,1.8$ or $18 \mathrm{mg} / \mathrm{kg}$ b.w.]. Sperm from cauda epididymis were sampled, counted and stored at $-80{ }^{\circ} \mathrm{C}$ for successive neutral Comet assay. Blood samples from all 6 animals per group were analyzed by Pig-a and MN assay. This decision was taken to fully exploit the available samples.

\section{Preparation of single cell suspensions}

A few days before sacrifice, $200 \mu \mathrm{l}$ of blood were harvested from the peri-orbital venous sinus of 6 mice/group/sex for repeated dose genotoxicity endpoints and collected into EDTA-coated microtainers (Becton Dickinson, CA, USA).

Blood aliquots were processed as follows: immediately upon collection, $30 \mu \mathrm{l}$ of each specimen were used to prepare slides for Comet assay, $60 \mu \mathrm{l}$ were transferred to tubes containing $100 \mu \mathrm{l}$ anticoagulant solution and were processed to be analysed by the Pig-a assay, and $60 \mu \mathrm{l}$ were

\footnotetext{
${ }^{1}$ OECD, Test No. 489, 2014: In Vivo Mammalian Alkaline Comet Assay, OECD Publishing, Paris. 
transferred to tubes containing $350 \mu \mathrm{l}$ anticoagulant solution, fixed with ultra-cold methanol and stored, in two aliquots, at $-80^{\circ} \mathrm{C}$ for the subsequent flow cytometric analysis of $\mathrm{MN}$ in erythrocytes.

Excised liver, kidney, duodenum, ovary and testis were immersed in mincing buffer (PBS containing $25 \mathrm{mM} \mathrm{Na} 2$ EDTA and DMSO10\%), epididymes were immersed in TNE buffer $(0.15 \mathrm{M} \mathrm{NaCl}, 0.01 \mathrm{M}$ Tris-HCl, $0.001 \mathrm{M} \mathrm{Na}_{2} \mathrm{EDTA}, \mathrm{pH} 7.4$ ) and kept on ice. Epididymes were cut in pieces in TNE buffer and sperm were released by pipetting, filtered and counted in a hemocytometer. Sperm suspensions were centrifuged, pellet was suspended in TNE buffer supplemented with $10 \%$ glycerol and stored at $-80^{\circ} \mathrm{C}$ until analysis by neutral Comet assay. Liver, kidney, ovary and testis were cut into small pieces and cells were mechanically isolated (Medimachine $\AA$, Becton Dickinson). Duodenum was rinsed with mincing buffer, cut longitudinally and scraped with a spatula. All cell suspensions except ovary were filtered through $50 \mu \mathrm{m}$ and centrifuged 8 minutes at 500 round per minute $(\mathrm{rpm})$ at $4^{\circ} \mathrm{C}$. Ovary cells were directly centrifuged 8 minutes at $800 \mathrm{rpm}$. Pellets were then resuspended in cold PBS.

\subsubsection{In vivo Comet assay}

Slides were prepared and scored by ENEA and ISS for males and females respectively, following the same protocol with slight changes, essentially related to staining and computerized image analysis systems that were laboratory specific because of a consolidated experience with each own equipment.

Thirty microliters of blood or cell suspension were mixed with $195 \mu \mathrm{l} 0.7 \%$ low melting point agarose (Bio-Rad Lab., Hercules, CA, USA) and $75 \mu \mathrm{l}$ of this suspension were seeded on slides pre-coated with $1 \%$ normal melting point agarose (Bio-Rad). Slides were covered with glass coverslips and kept on a cold plate until gel solidification. Two slides per tissue were produced for each animal. After coverslip removal, the slides were put into lysis solution $\left(2.5 \mathrm{M} \mathrm{NaCl}, 0.1 \mathrm{M} \mathrm{Na}_{2} \mathrm{EDTA}, 10 \mathrm{mM}\right.$ Tris pH 10, extemporarily added with DMSO10\% and $1 \%$ Triton X-100), and kept overnight at $4{ }^{\circ} \mathrm{C}$. The slides were placed in an electrophoresis tank and DNA was allowed to unwind at $4{ }^{\circ} \mathrm{C}$ for 25 min in fresh electrophoresis buffer $\left(0.3 \mathrm{M} \mathrm{NaOH}, 1 \mathrm{mM} \mathrm{Na}{ }_{2} \mathrm{EDTA}, \mathrm{pH}>13\right)$. Electrophoresis was carried out at $4{ }^{\circ} \mathrm{C}$ for $25 \mathrm{~min}, 27 \mathrm{~V}(0.8 \mathrm{~V} / \mathrm{cm}), 300 \mathrm{~mA}$. The slides were then washed for $5 \mathrm{~min}$ into a neutralizing solution (0.4 M Tris- $\mathrm{HCl}, \mathrm{pH} 7.5)$, fixed with ethanol for $5 \mathrm{~min}$ and dried for conservation. Coded slides from males were stained with ethidium bromide $(12 \mu \mathrm{g} / \mathrm{ml}$ in PBS) and examined with an Olympus fluorescence microscope by a computerized image analysis system (Delta Sistemi, Italy). Coded slides from females were stained with Gel RED Nucleic Acid Gel Stain used according to supplier instructions (Biotium) and examined with a Nikon fluorescence microscope by a computerized image analysis system (LUCIA Comet Assay ${ }^{\top \mathrm{M}}$, Laboratory Imaging, Prague). A total of 150 cells, from two different slides were scored for each tissue/animal. The percentage of total DNA in the Comet tail (tail intensity, $\% \mathrm{TI}$ ), tail length and Olive Tail Moment (OTM) were recorded. Tail intensity was used as the parameter to quantify DNA damage. Results are given as the \%TI mean value of the medians of the two slides for each tissue/animal according to OECD TG $489^{1}$. Hedgehogs, identified by visual inspection or when their tail intensity value was higher than $80 \%$, were separately recorded and their percentage was calculated for each tissue/animal. Hedgehogs, have been suggested as indicators of cytotoxicity, although their etiology is uncertain. It has been hypothesized that they can be caused by substance-related cytotoxicity, mechanical/enzyme-induced damage initiated during sample preparation and/or an extreme effect of test chemical genotoxicity (OECD TG $489^{1}$ ).

\section{Neutral Comet assay on sperm}

Frozen sperm aliquots were thawed at room temperature and immediately washed with PBS. After centrifugation (3000 rpm, $3 \mathrm{~min}$ ) pellets were resuspended in PBS to prepare slides as described for alkaline Comet assay. To allow the migration of the extremely compacted DNA of sperm, a decondensation step after lysis (30 min in $10 \mathrm{mM}$ dithiothreitol (DTT) (Sigma-Aldrich) in lysis solution) was employed. After this step, the slides were placed in an electrophoresis tank containing TBE buffer (2 $\mathrm{mM} \mathrm{Na} \mathrm{EDTA}_{2} 90 \mathrm{mM}$ Tris, $90 \mathrm{mM}$ boric acid; $\mathrm{pH} 8$ ) at $4{ }^{\circ} \mathrm{C}$ and left there for $20 \mathrm{~min}$.

\footnotetext{
${ }^{1}$ OECD, Test No. 489, 2014: In Vivo Mammalian Alkaline Comet Assay, OECD Publishing, Paris. 
Electrophoresis was carried out at $4{ }^{\circ} \mathrm{C}$ for 7 minutes, $27 \mathrm{~V}(0.8 \mathrm{~V} / \mathrm{cm})$ and $10 \mathrm{~mA}$. Slides were then dried, stained and analyzed as for alkaline Comet assay.

\subsubsection{Pig-a gene mutation assay}

Within $24 \mathrm{~h}$ after sampling, peripheral blood was processed according to the procedures described in the MutaFlowPLUS Kit ${ }^{\circledR}$ (Mouse Blood; Litron Laboratories, NY, USA) and as described by Dertinger et al. 2011. Most leukocytes and platelets were depleted by density-gradient centrifugation with Lympholyte-Mammal solution (Cedarlane, NC, USA) and the resulting erythrocyte-enriched samples were labelled with phycoerythrin (PE)-anti-CD24 and PE-anti-CD61 (from the MutaFlow kit), followed by labelling with anti-PE magnetic microbeads (Miltenyi Biotech, CA, USA). After washing, cells were mixed with $1 \mathrm{ml}$ kit buffer solution. A small fraction of the labelled cells and beads suspension was reserved as a 'pre-column' sample for subsequent flow cytometric analysis. The main part of the labelled cells and beads suspension was processed on an LS magnetic column (Miltenyi) mounted onto a quadroMACS magnet (Miltenyi). CD24-positive wild-type RBCs, and CD61-positive platelets were retained on the column and the flow through fraction, consisting of enriched CD24-deficient (Pig-a mutant) RBCs, was collected as a 'post-column' sample for subsequent flow cytometric analysis. Before the analyses, both the 'pre-' and 'post-column' samples were stained with a solution containing SYTO13 $\AA$ dye (from the MutaFlow kit), to allow the distinction between reticulocytes and mature erythrocytes, and CountBright ${ }^{\mathrm{TM}}$ absolute counting beads (Life Technologies, CA, USA).

An instrument calibration standard was prepared by creating a specimen consisting of $\sim 50 \%$ wildtype and $50 \%$ mutant-mimicking cells processed in the same way as the experimental samples but not incubated with antibodies. The flow cytometric analysis was performed on a FACSCalibur flow cytometer (Becton Dickinson) equipped with a $488 \mathrm{~nm}$ laser using the CellQuest software (Becton Dickinson). For each animal, the frequencies of Pig-a mutant RBCs and RETs were calculated from the cell equivalents analysed using the ratios of the CountBright beads to total RBCs and RETs in the 'precolumn' sample and the ratios of CountBright beads to enriched mutant total RBCs and mutant RETs in the 'post-column' sample. Additionally, the percentage of RETs, identified as SYTO13 ${ }^{\circledR}$ positive cells, was recorded, with reduced \%RETs indicating cytotoxicity.

\subsubsection{Micronucleus test}

Peripheral blood was processed according to the procedure described in the Mouse Blood MicroFlow Micronucleus Analysis Kit ${ }^{\circ}$ (Litron Laboratories). Prior to analysis, samples were washed and incubated with RNase, anti-CD71-FITC (anti-transferrin receptor antibody that labels RETs), and antiCD61-PE (anti-platelet antibody that labels platelets) for $30 \mathrm{~min}$ at $4^{\circ} \mathrm{C}$ followed by $30 \mathrm{~min}$ at room temperature. Immediately before analysis, the samples were re-suspended in an appropriate amount of kit-supplied DNA staining solution. The flow cytometer photomultiplier and compensation tubes were adjusted using a kit-supplied malaria-infested blood sample. This biological standard was used to set the optimal voltage for resolving parasitized (Micronucleus-like) RETs, and hence the position of the quadrant delineating normochromatic red blood cells (NCEs) and RETs with and without micronuclei. The samples were analyzed on a Becton Dickinson FACSCalibur flow cytometer using the CellQuest software. Twenty thousand RETs were analyzed per blood sample. Again, the \%RETs, identified as CD-71 positive cells, was recorded as an index for cytotoxicity. In each measure session, positive and negative biological standards provided with the kit were analyzed in order to verify the reliability of measurements and the values of \%RETs and \%MN-RETs obtained were always within the ranges recommended.

\subsubsection{Biomarker analysis}

\section{Hormones serum markers}

Selected endocrine/reproductive biomarkers, namely $17 \beta$ estradiol (E2), testosterone (T), tetraiodothyronine (T4) and thyroid stimulating hormone (TSH), as well as markers of liver and kidney function - alanine aminotransferase (ALT), aspartate aminotransferase (AST), creatinine (CRE) and 
blood urea nitrogen (BUN) - were measured in serum of treated and CTRL mice of both sexes and in dams, in order to evaluate functional effects on related systems. Blood samples collected prior to sacrifice, were left to coagulate at room temperature for $1 \mathrm{~h}$ and then centrifuged for $15 \mathrm{~min}$ at 2000 rpm twice in a cooled bench-top centrifuge (Microlite Microfuge, Thermo Electron Corporation). After centrifugation, the serum was stored at $-80^{\circ} \mathrm{C}$ until use. E2, T4 and TSH serum levels were measured by commercial ELISA kits from Cusabio (Cusabio Biotech Ltd., MD, USA) whereas T was measured by a commercial ELISA kit from BioVendor (Brno, Czech Republic). Each kit provided a standard solution of the hormone to assay and 5 to 6 dilutions were prepared to derive a standard curve and define the range of linearity of each test. For all the analyses the manufacturer's instructions were followed. Each sample was assessed in duplicate. At the end of the tests, absorbance was read at $450 \mathrm{~nm}$ on a VICTOR3 Multilabel microplate reader (Perkin Elmer, MA, USA). By the software GraphPad Prism 6.0 (GraphPad Software Inc., CA, USA) we derived the unknown hormone concentrations in samples by using the standard curve of each hormone. AST, ALT, CRE and BUN were measured by the automatic analyser Keylab Analyser (BPC Biosed s.r.l., Italy).

\section{Oxidative stress biomarkers in brain}

The effects of BEA and ENNB treatment on oxidative stress status in brain were evaluated by measurement of the amount of ROS and GSH. Brains of treated and CTRL male and female mice, as well as of dams, were excised at sacrifice, flash frozen in liquid nitrogen and stored at $-80^{\circ} \mathrm{C}$ until use. After thawing, each brain was separated in two aliquots and weighed.

To assay GSH levels, one aliquot of brain tissue was added with 10 fold the volume of a $5 \% 5$ sulfosalicylic acid solution to deproteinize the samples which were then homogenized by the Miccra D1 homogenizer (ART-moderne Labortechnik, Germany) on ice. Lysates were centrifuged at $3000 \mathrm{rpm}$ for $15 \mathrm{~min}$ at $4{ }^{\circ} \mathrm{C}$, then supernatants were collected. 1:5 dilution of each sample was assayed by the Glutathione Assay Kit (Sigma-Aldrich, Italy) containing also a standard solution of GSH which was serially diluted to derive a standard curve. Manufacturer's instruction was followed to prepare the kinetic mixture; at the end of incubation, absorbance was read at $405 \mathrm{~nm}$ on a VICTOR3 Multilabel microplate reader (Perkin Elmer) each minute for five minutes. Each sample was assayed in duplicate. The increase in absorbance was recorded to derive GSH amount developed in $\mu$ moles per minute by the standard curve designed with Graph Pad Prism 6.0.

To assay ROS amount, the other half of each brain tissue sample was added $400 \mu$ PBS and homogenized by the Miccra D-1 homogenizer (ART-moderne Labortechnik) on ice. Lysates were centrifuged at $3000 \mathrm{rpm}$ for $15 \mathrm{~min}$ at $4{ }^{\circ} \mathrm{C}$, then supernatants were collected. Total protein concentration in each sample was assessed by the Pierce BCA Protein Assay Kit (Thermo Fisher Scientific, MA, USA) reading absorbance at $560 \mathrm{~nm}$ on a NanoDrop (Thermo Fisher) instrument. 200 $\mu \mathrm{l}$ of each sample were added with $10 \mu \mathrm{l} 2^{\prime}, 7^{\prime}$-Dichlorofluorescin diacetate $500 \mu \mathrm{M}$, a cell-permeable non-fluorescent probe which becomes fluorescent upon oxidation. Following incubation for $30 \mathrm{~min}$ at room temperature, fluorescent intensity was recorded by excitation at $485 \mathrm{~nm}$ and emission at 535 $\mathrm{nm}$ by a VICTOR3 Multilabel microplate reader (Perkin Elmer). Each sample was assayed in duplicate. Recorded fluorescence signals were normalized to total protein content.

\subsubsection{Immunotoxicity endpoints in male and female mice}

\section{Antibody determination}

To assess serum antibody levels, blood was left to coagulate at room temperature for 1 hour and then centrifuged for $15 \mathrm{~min}$ at $2000 \mathrm{rpm}$ in a cooled bench-top centrifuge (Microlite Microfuge, Thermo Electron Corporation). After centrifugation, serum samples were snap frozen in liquid nitrogen and stored at $-80^{\circ} \mathrm{C}$ until analysis. Total serum IgG, IgA and IgM antibody isotypes were evaluated by commercially available kits, following the manufacturing instructions (Mouse IgG total Ready-SET-Go, Mouse IgA total Ready-SET-Go, Mouse IgM total Ready-SET-Go, Affimetrix, eBioscience). Individual serum samples were diluted 1:50000, 1:5000, 1:50000, for IgG, IgA, IgM determination, respectively, and tested in duplicate. 


\section{Blood counts}

Whole blood samples in EDTA, as anti-coagulant agent, were collected a few days before sacrifice, to perform blood count analysis within few hours by Simply Cell cell counter (BPC BioSed). The following blood cell populations were evaluated and expressed as number of cells/ $\mu$ l: white blood cells (WBC), lymphocytes (LYMPH), granulocytes (GRAN), less frequently occurring cells correlating to monocytes, eosinophils, basophils, blasts and other precursor white cells (MID), RBC, and platelets (PLT).

\section{Lymphoid organ collection}

At the end of treatments, the animals were sacrificed and spleen, thymus and MLN were collected. Absolute and relative weights of the three lymphoid organs were recorded. Spleens were used to obtain cells for determination of in vitro immunotoxicity endpoints.

\section{Spleen cell suspension and in vitro cultures}

Spleens were mechanically disrupted on a cell strainer. After RBC lysis, the final cell suspension was used both for $\mathrm{T}$ cell and macrophage analysis.

To assess cytokine production (interferon (IFN)y and interleukin (IL)-10), $2 \times 10^{6}$ cells/well were seeded in 24 well plates in presence or absence of anti-CD3 (pre-coated) and anti-CD28, as polyclonal stimuli. After $48 \mathrm{~h}$ the culture supernatants were collected and stored at $-20{ }^{\circ} \mathrm{C}$ until cytokine evaluation.

To study the innate compartment, spleen cells were suspended in high-glucose DMEM plus $10 \%$ FBS and $10 \times 10^{6}$ cells/well were cultured in 24 wells plates; after three hours of incubation at $37{ }^{\circ} \mathrm{C}$, nonadherent cells were removed. Adherent cells (macrophages) were incubated with or without LPS at the final concentration of $1 \mu \mathrm{g} / \mathrm{ml}$. After $48 \mathrm{~h}$ of incubation, supernatants were collected and NO levels were evaluated.

\section{NO determination}

NO production was evaluated in culture supernatants by Greiss reagent, adding in 96 wells plate an equal volume of individual supernatants or standards, or quality CTRLs. A double volume of Greiss reagent was added to all wells and the absorbance was read at $550 \mathrm{~nm}$. Each individual sample was tested in duplicate.

\section{Cytokine determination}

IFNy e IL-10 levels in culture supernatants were determined by commercially available kits (Affimetrix, eBioscience) according to manufacturing instructions. Each individual sample was tested in duplicate.

\section{FACS analysis}

To analyse the distribution of T lymphocyte subpopulations in the spleen, cytofluorimetric analysis was performed by staining $6 \times 10^{6}$ cells with the following fluorochrome conjugated antibodies: anti-mouse CD3-APC, anti-mouse CD4-PE, anti-mouse CD8-FITC, anti-mouse CD45R(B220)-PerCP-Cy5.5 (all by Affimetrix, eBioscience). Isotype CTRLs were used for setting purposes. All the samples were analyzed by FACSCanto cytofluorimeter (Becton Dickinson).

\subsection{Statistical analysis}

Data analysis was performed using Stata ver.14 (StataCorp, Lakeway Drive College Station, Texas, USA) and GraphPah PRISM 6 (GraphPad Software Inc., La Jolla, CA, USA). BEA treated groups were compared with olive oil CTRL groups and ENNB treated groups were compared with olive oil + DMSO6\% CTRL groups. For all the endpoints not related to genotoxicity, the statistical significance was assessed by the non parametric Kruskal-Wallis test, due to the not normal distribution of data, followed by the post-hoc Dunn's test comparison among treated groups and CTRLs, where appropriate. For genotoxicity data, One-way ANOVA was performed followed by the post-hoc 
Dunnett's test comparison among treated groups and CTRLs, where appropriate. For categorical histopathological data, Pearson's Chi Square test was performed. Significance level was set at $p<0.05$ for all the analyses.

\section{Results}

\subsection{Acute in vivo genotoxicity study}

\subsubsection{BEA - Clinical observation and toxicity effects}

No mortality, no clinical signs of toxicity and no significant reduction in body weight were underlined after 3 oral administrations (Table 4). Despite the presence of some lipid droplets in hepatocytes at the middle dose $(100 \mathrm{mg} / \mathrm{kg})$, no macroscopic effects on any organs were noticed in any group.

Table 4: $\quad$ Body weight gain at $24 \mathrm{~h}$ and $48 \mathrm{~h}$ of male mice after 3-day oral administration with different doses of BEA ( 0 - colza oil+DMSO5\%, 50, 100 and $200 \mathrm{mg} / \mathrm{kg} \mathrm{b.w.} \mathrm{per} \mathrm{day).} \mathrm{Results}$ are expressed as the mean $( \pm S D)$ of 5 animals.

\begin{tabular}{llcc}
\hline \multirow{2}{*}{ Treatment } & \multirow{2}{*}{ Dose (mg/kg b.w.) } & \multicolumn{2}{c}{ b.w. gain (mg) } \\
\cline { 3 - 4 } & & $\mathbf{2 4 h}$ & $\mathbf{4 8 h}$ \\
\hline CTRL & Colza oil + DMSO5\% & $-0.16 \pm 1.09$ & $0.8 \pm 0.61$ \\
BEA & $\mathbf{5 0}$ & $0.68 \pm 0.70$ & $0.8 \pm 0.70$ \\
& $\mathbf{1 0 0}$ & $-0.1 \pm 1.16$ & $1.16 \pm 0.43$ \\
& $\mathbf{2 0 0}$ & $0.3 \pm 0.73$ & $1.82 \pm 0.59$ \\
\hline
\end{tabular}

In order to evaluate if BEA induced systemic toxicity effect in mice after 3 oral gavages in $48 \mathrm{~h}$, we performed histopathological analysis of liver slides. We observed microvesicular steatosis in $3 / 5,3 / 5$, $0 / 5$ and $2 / 5$ animals for CTRL and 50, 100 and $200 \mathrm{mg} / \mathrm{kg} \mathrm{b.w.} \mathrm{groups} \mathrm{respectively} \mathrm{(Table} \mathrm{5).} \mathrm{The}$ presence of microvesicular steatosis seemed due to colza oil use as vehicle. BEA induced slight liver toxicity effect at all doses tested, with presence of mitotic cells and clarification, in particular at the middle dose. The presence of clarification, which may represent a stage of reversible cellular suffering, can mask the presence of lipid microvesicular steatosis. The absence of dose-effect relationship in liver toxicity parameters (clarification) suggested an absence of liver toxicity effect of BEA in mice implying that MTD was not reached. However, it should be noted that the highest dose tested largely exceeded (about $2 \times 10^{6}$ times greater) the highest acute human exposure estimates. 
Table 5: $\quad$ Incidence for each histopathological endpoint analyzed on liver of male mice after 3day oral administration with different doses of BEA (0 - colza oil+DMSO5\%, 50, 100 and 200 $\mathrm{mg} / \mathrm{kg}$ b.w. per day)

\begin{tabular}{|c|c|c|c|c|c|c|}
\hline Treatment & $\begin{array}{c}\text { Dose } \\
\text { (mg/kg b.w.) }\end{array}$ & Steatosis ${ }^{a}$ & Inflammation $^{\mathrm{a}}$ & Clarification $^{a}$ & Necrosis & Mitosis $^{b}$ \\
\hline \multirow[t]{5}{*}{ CTRL } & $\begin{array}{l}\text { Colza oil + } \\
\text { DMSO5\% }\end{array}$ & 1 (Microvesicular) & 0 & 0 & 0 & 0 \\
\hline & & 0 & 0 & 0 & 0 & 0 \\
\hline & & 1 (Microvesicular) & 0 & 0 & 0 & 0 \\
\hline & & 1 (Microvesicular) & 0 & 0 & 0 & 0 \\
\hline & & 0 & 0 & 0 & 0 & 0 \\
\hline \multirow[t]{15}{*}{ BEA } & 50 & 1 (Microvesicular) & 0 & 0 & 0 & 0 \\
\hline & & 0 & 1 (eosinophile) & 3 & 0 & 2 \\
\hline & & 1 (Microvesicular) & 0 & 0 & 0 & 4 \\
\hline & & 1 (Microvesicular) & 0 & 2 & 0 & 0 \\
\hline & & 0 & 0 & 2 & 0 & 0 \\
\hline & 100 & 0 & 0 & 0 & 0 & 0 \\
\hline & & 0 & 0 & 1 & 0 & 1 \\
\hline & & 0 & 0 & 0 & 0 & 3 \\
\hline & & 0 & 0 & 1 & 0 & 4 \\
\hline & & 0 & 0 & 2 & 0 & 6 \\
\hline & 200 & 1 (Microvesicular) & 0 & 0 & 0 & 0 \\
\hline & & 0 & 0 & 3 & 0 & 0 \\
\hline & & 0 & 0 & 2 & 0 & 1 \\
\hline & & 2 (Microvesicular) & 0 & 0 & 0 & 0 \\
\hline & & 0 & 0 & 0 & 0 & 0 \\
\hline
\end{tabular}

${ }^{\mathrm{a}}$ five grades between 1 and 5 were used - minimal (1) , slight (2), moderate (3), marked (4) or severe (5). ${ }^{\mathrm{b}}$ Number of mitotic cells $/ \mathrm{mm}^{2}$

\subsubsection{BEA - Alkaline Comet assay}

\section{First study}

Figures 3-4 and Table 6 present the results obtained by the Comet assay with BEA in the first experiment. DNA damage, expressed as \% of tail intensity, obtained for each animal in each organ/tissue is presented. The \% of hedgehogs is also presented. Hedgehogs are heavily damaged cells which could not be reliably analyzed by the software. Hedgehogs could be due to DNA damage, apoptosis or necrosis and their increase due to the test chemical should be investigated and interpreted with care $\left(\mathrm{OECD}\right.$ TG $\left.489^{1}\right)$. Results in the negative CTRL group show a low level of DNA migration in the different organs. The means of \%TI medians in the negative CTRL groups ranged from $0.08 \%$ (blood) up to $1.33 \%$ (colon) whereas in the positive group treated with $80 \mathrm{mg} / \mathrm{kg}$ b.w. MMS values ranged from $7.67 \%$ (bone marrow) up to $18.05 \%$ (kidney). The values of the MMS group were significantly increased in all tissues over those of the negative CTRL group. MMS also increased the frequency of hedgehogs. The results after BEA treatment at 50,100 and $200 \mathrm{mg} / \mathrm{kg}$ showed a low level of DNA migration (lower than 1\%) and frequency of hedgehogs. No significant differences with negative CTRL were detected.

\footnotetext{
${ }^{1}$ OECD, Test No. 489, 2014: In Vivo Mammalian Alkaline Comet Assay, OECD Publishing, Paris. 

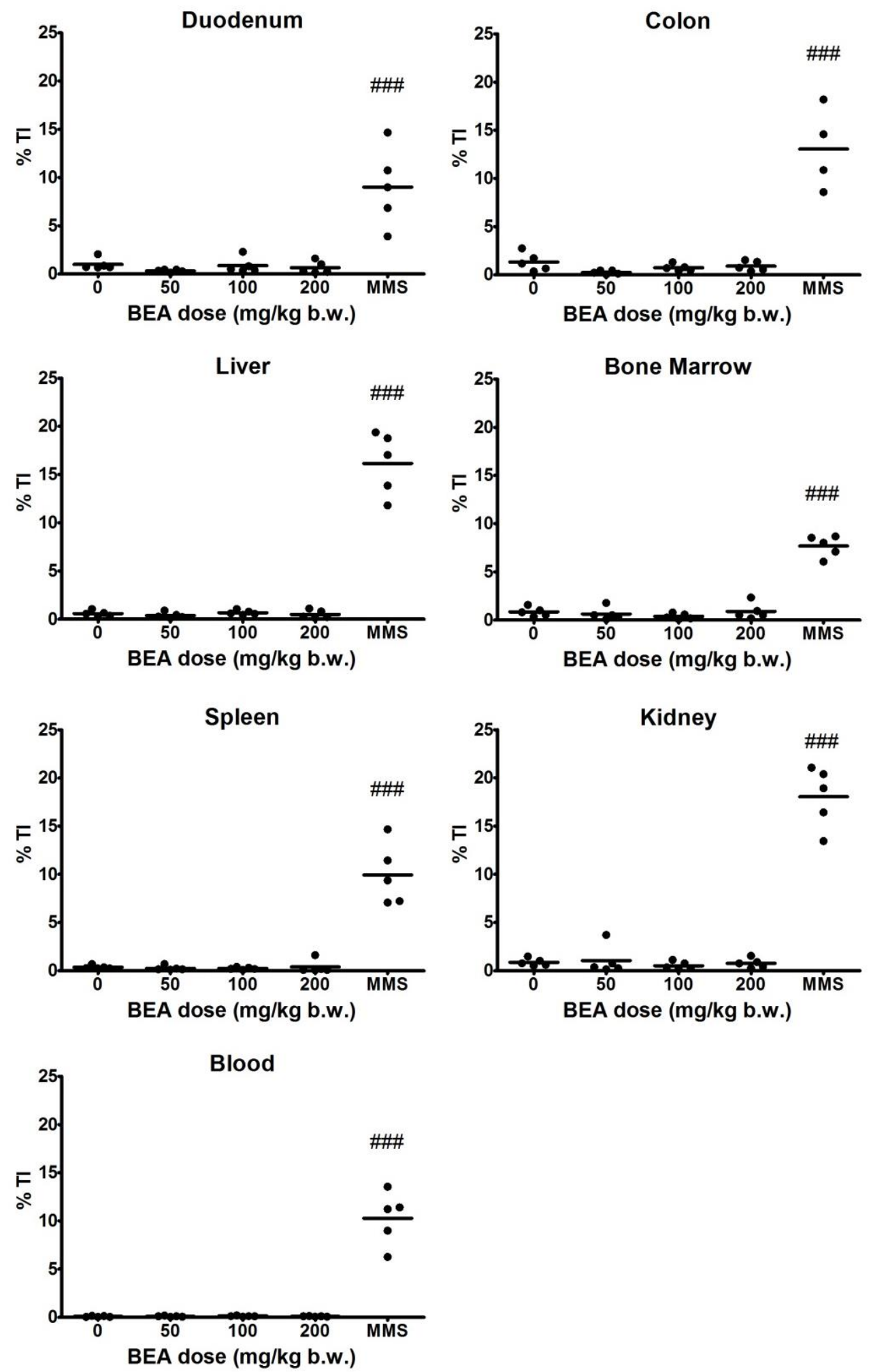

Figure 3: Individual results ( 5 animals/group) obtained in the Comet assay in different tissues after 3-day oral administration with different doses of BEA (0 - colza oil+DMSO5\%, 50, 100 and $200 \mathrm{mg} / \mathrm{kg}$ b.w. per day). DNA damage is expressed as \% of tail DNA intensity. MMS treatment was used for positive CTRL group. Lines indicate mean of medians of percentage tail intensity in each group. \#\#\# $\mathrm{p}<0.001$. 

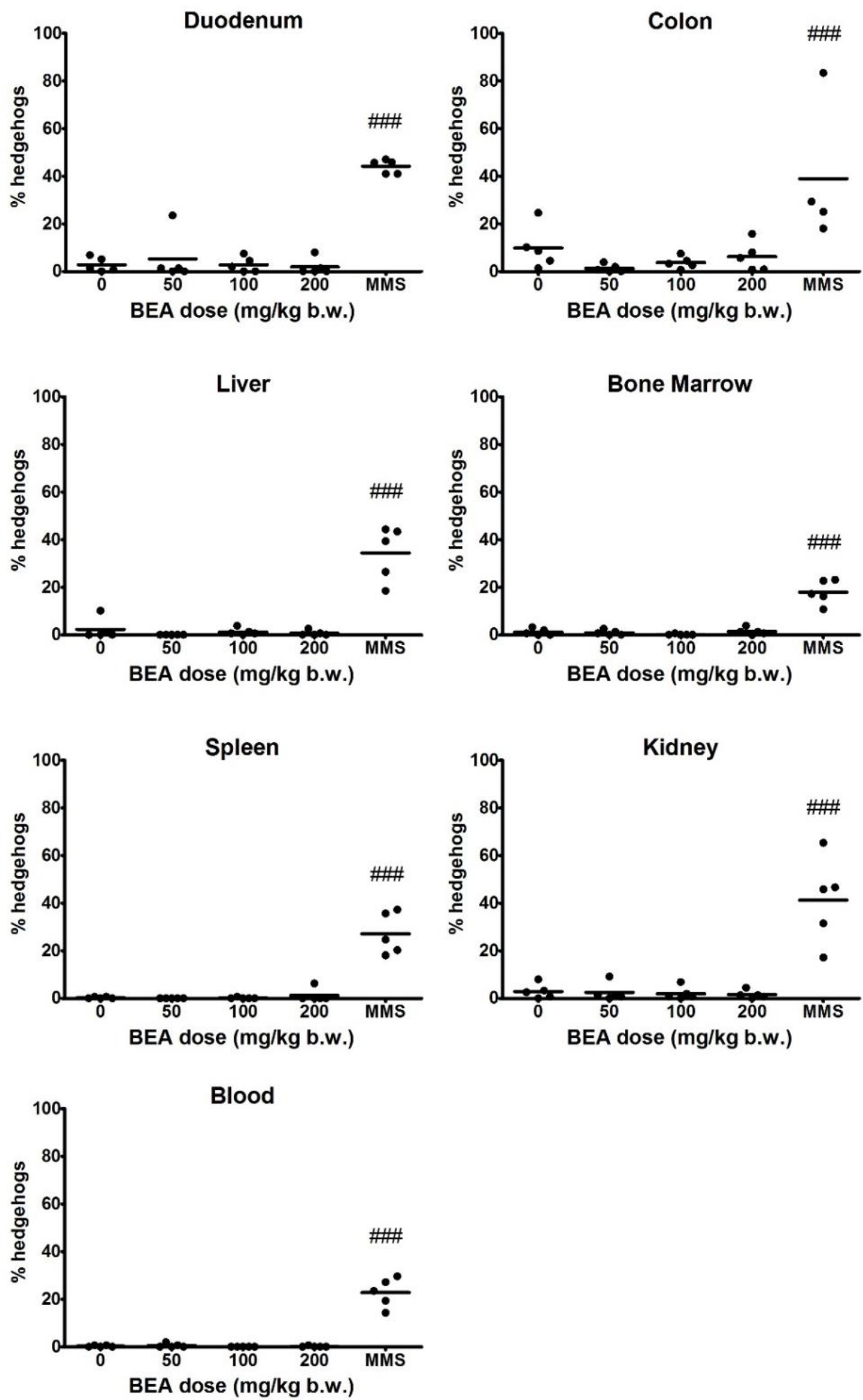

Figure 4: Individual results ( 5 animals/group) obtained in the Comet assay in different tissues after 3-day oral administration with different doses of BEA (0 - colza oil+DMSO5\%, 50, 100 and $200 \mathrm{mg} / \mathrm{kg}$ b.w. per day). MMS treatment was used for positive CTRL group. Lines indicate mean of $\%$ hedgehogs per groups. \#\#\# $p<0.001$. 
Table 6: Alkaline Comet assay in different organs/tissues of male mice after 3-day oral administration with different doses of BEA (0 - colza oil+DMSO5\%, 50, 100 and $200 \mathrm{mg} / \mathrm{kg}$ b.w. per day) or with MMS as positive CTRL. For each experimental group the mean of medians \% DNA in tail and \% hedgehogs are reported.

\begin{tabular}{|c|c|c|c|c|}
\hline Tissues/organs & Treatment & $\begin{array}{c}\text { Dose } \\
(\mathrm{mg} / \mathrm{kg} \mathrm{b.w./d})\end{array}$ & $\begin{array}{c}\% \text { TI } \\
\text { ( } \pm \text { SD) }\end{array}$ & $\begin{array}{c}\text { \% Hedgehogs } \\
( \pm \text { SD) }\end{array}$ \\
\hline \multirow{5}{*}{ Blood } & CTRL & Colza oil+ DMSO5\% & $0.08 \pm 0.05$ & $0.27 \pm 0.55$ \\
\hline & BEA & 50 & $0.09 \pm 0.05$ & $0.53 \pm 0.91$ \\
\hline & & 100 & $0.12 \pm 0.05$ & $0.00 \pm 0.00$ \\
\hline & & 200 & $0.09 \pm 0.03$ & $0.013 \pm 0.42$ \\
\hline & MMS & 80 & 10.28 土 2.76\#\#\# & 23.16 土 6.24\#\#\# \\
\hline \multirow{5}{*}{ Bone marrow } & CTRL & Colza oil+ DMSO5\% & $0.86 \pm 0.47$ & $1.19 \pm 2.67$ \\
\hline & BEA & 50 & $0.64 \pm 0.66$ & $0.92 \pm 1.44$ \\
\hline & & 100 & $0.37 \pm 0.29$ & $0.13 \pm 0.51$ \\
\hline & & 200 & $0.89 \pm 0.86$ & $1.45 \pm 2.45$ \\
\hline & MMS & 80 & $7.67 \pm 1.09 \# \# \#$ & $18.21 \pm 7.32 \# \# \#$ \\
\hline \multirow{5}{*}{ Liver } & CTRL & Colza oil+ DMSO5\% & $0.58 \pm 0.29$ & $2.47 \pm 4.40$ \\
\hline & BEA & 50 & $0.39 \pm 0.31$ & $0.00 \pm 0.00$ \\
\hline & & 100 & $0.68 \pm 0.24$ & $1.32 \pm 2.20$ \\
\hline & & 200 & $0.50 \pm 0.43$ & $0.67 \pm 1.94$ \\
\hline & MMS & 80 & $16.17 \pm 3.25 \# \# \#$ & $35.84 \pm 12.10 \# \# \#$ \\
\hline \multirow{5}{*}{ Kidney } & CTRL & Colza oil+ DMSO5\% & $0.88 \pm 0.38$ & $2.98 \pm 3.77$ \\
\hline & BEA & 50 & $1.05 \pm 1.51$ & $2.60 \pm 3.87$ \\
\hline & & 100 & $0.53 \pm 0.41$ & $2.09 \pm 4.07$ \\
\hline & & 200 & $0.77 \pm 050$ & $1.57 \pm 2.48$ \\
\hline & MMS & 80 & 18.05 土 3.13\#\#\# & $43.82 \pm 21.50 \# \# \#$ \\
\hline \multirow{5}{*}{ Spleen } & CTRL & Colza oil+ DMSO5\% & $0.36 \pm 0.19$ & $0.27 \pm 0.69$ \\
\hline & BEA & 50 & $0.26 \pm 0.24$ & $0.00 \pm 00$ \\
\hline & & 100 & $0.23 \pm 0.13$ & $0.13 \pm 0.51$ \\
\hline & & 200 & $0.41 \pm 0.68$ & $1.32 \pm 3.20$ \\
\hline & MMS & 80 & $9.95 \pm$ 3.19\#\#\# & 28.02 土 9.23\#\#\# \\
\hline \multirow{5}{*}{ Duodenum } & CTRL & Colza oil+ DMSO5\% & $0.99 \pm 0.59$ & $2.85 \pm 3.68$ \\
\hline & BEA & 50 & $0.32 \pm 0.14$ & $6.25 \pm 9.98$ \\
\hline & & 100 & $0.86 \pm 0.82$ & $2.85 \pm 4.51$ \\
\hline & & 200 & $0.61 \pm 0.58$ & $3.60 \pm 6.30$ \\
\hline & MMS & 80 & $9.02 \pm 4.06 \# \# \#$ & $44.20 \pm 4.88 \# \# \#$ \\
\hline \multirow{5}{*}{ Colon } & CTRL & Colza oil+ DMSO5\% & $1.33 \pm 0.95$ & $10.61 \pm 10.19$ \\
\hline & BEA & 50 & $0.25 \pm 0.18 \#$ & $1.32 \pm 2.33$ \\
\hline & & 100 & $0.73 \pm 0.35$ & $3.72 \pm 3.27$ \\
\hline & & 200 & $0.90 \pm 0.51$ & $6.99 \pm 8.16$ \\
\hline & MMS & 80 & $12.97 \pm 4.28 \# \# \#$ & $36.62 \pm 31.14 \# \# \#$ \\
\hline
\end{tabular}

$\# \# \# p<0.001$

\section{Second study}

In the first study, the electrophoresis condition (voltage $0.37 \mathrm{~V} / \mathrm{cm}$ ) applied was not optimal to reveal a low level of DNA damage. Even if the results of this study are to be considered valid since the positive CTRL values were always statistically increased over negative CTRL values, we decided to carry out a second experiment applying more sensitive conditions in order to detect small effects possibly missed in the first study. In this second experiment DNA damage was assessed, after 2 oral administrations of BEA, in two relevant organs, the liver, because it is the most active organ in 
metabolism of substances and also frequently a target for carcinogenicity and the bone marrow, to evaluate a systemic effect.

In the second study, the positive CTRL MMS significantly increased the DNA migration in bone marrow and liver, whereas we did not observe any effect of BEA in the two organs (Table 7 and Fig. 5). The frequency of hedgehogs induced by MMS in liver and bone marrow in the second experiment was lower than in the first experiment. This could be due to the increased voltage applied which determined an increase of DNA migration in cells with heavily damaged DNA resulting in comets too diffuse to be scored.

Table 7: Alkaline Comet assay in liver and bone marrow of male mice after 2 oral administrations with different doses of BEA (0 - colza oil+DMSO5\%, 50, 100 and $200 \mathrm{mg} / \mathrm{kg}$ b.w per day) or with MMS as positive CTRL. For each experimental group the mean of medians $\%$ DNA in tail and \% hedgehogs are reported.

\begin{tabular}{|c|c|c|c|c|}
\hline Tissues/organs & Treatment & $\begin{array}{c}\text { Dose } \\
(\mathrm{mg} / \mathrm{kg} \mathrm{b.w./d)}\end{array}$ & \%TI (土 SD) & $\begin{array}{c}\text { \% Hedgehogs } \\
\text { ( } \pm \text { SD) }\end{array}$ \\
\hline \multirow{5}{*}{ Liver } & CTRL & Colza oil+ DMSO5\% & $1.62 \pm 0.40$ & $1.80 \pm 1.10$ \\
\hline & BEA & 50 & $0.41 \pm 0.08$ & $1.60 \pm 1.67$ \\
\hline & & 100 & $1.64 \pm 1.02$ & $2.40 \pm 1.82$ \\
\hline & & 200 & $1.17 \pm 0.42$ & $2.20 \pm 1.64$ \\
\hline & MMS & 80 & $13.79 \pm 2.94 \# \# \#$ & $10.00 \pm 1.58 \# \# \#$ \\
\hline \multirow{5}{*}{ Bone marrow } & CTRL & Colza oil+ DMSO5\% & $0.67 \pm 0.18$ & $0.80 \pm 0.84$ \\
\hline & BEA & 50 & $0.93 \pm 0.60$ & $1.60 \pm 1.14$ \\
\hline & & 100 & $0.90 \pm 0.35$ & $1.40 \pm 1.67$ \\
\hline & & 200 & $1.01 \pm 0.66$ & $1.20 \pm 1.30$ \\
\hline & MMS & 80 & $2.99 \pm 1.05 \# \# \#$ & $4.20 \pm 1.48 \# \# \#$ \\
\hline
\end{tabular}

$\# \# \# p<0.001$ 

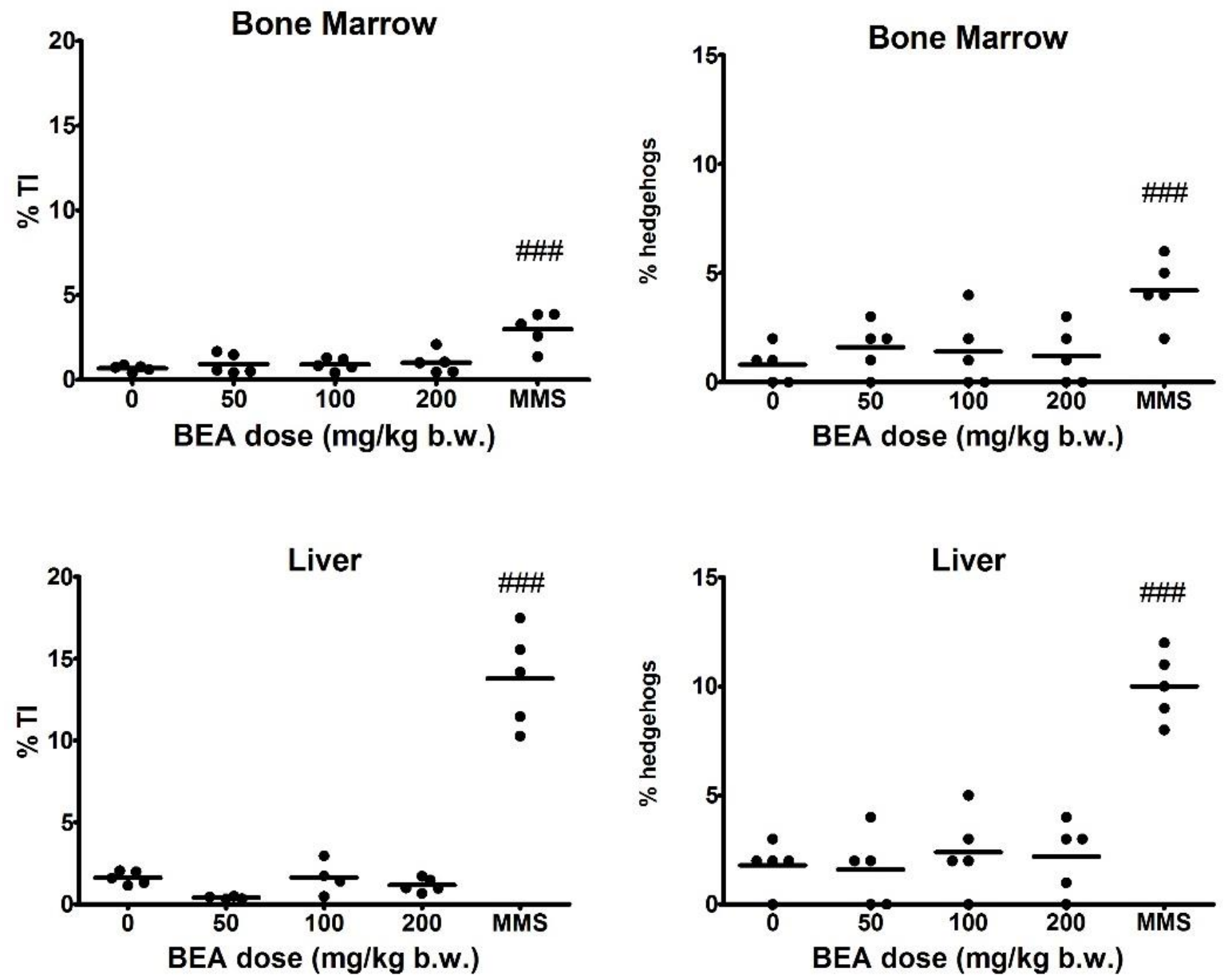

Figure 5: Individual results ( 5 animals/group) obtained in the Comet assay in different tissues after 2 oral treatments with different doses of BEA (0 - colza oil+DMSO5\%, 50, 100 and 200 $\mathrm{mg} / \mathrm{kg}$ b.w. per day). DNA damage is expressed as \% of tail DNA intensity; \% of hedgehogs is also shown. MMS treatment was used for positive CTRL group. Lines indicate mean of medians of percentage tail intensity and hedgehogs. \#\# $\mathrm{p}<0.001$.

\subsubsection{BEA - Micronucleus tests in colon}

The in vivo colon MN assay in mice provides information on the frequencies of micronucleated, mitotic and apoptotic cells. BEA did not increase micronucleated, mitotic or apoptotic cells formation in colon between 50 and $200 \mathrm{mg} / \mathrm{kg}$ b.w. (Fig. 6). The clastogen MMS did not increase MN formation in colon cells, but significantly increased apoptotic cells. We also integrated results obtained by the clastogenic compound dimethylhydrazine (DMH, Sigma) to demonstrate the capacity of this test to detect chromosome damage. DMH induced an increase of micronucleated and apoptotic cells in colon. 

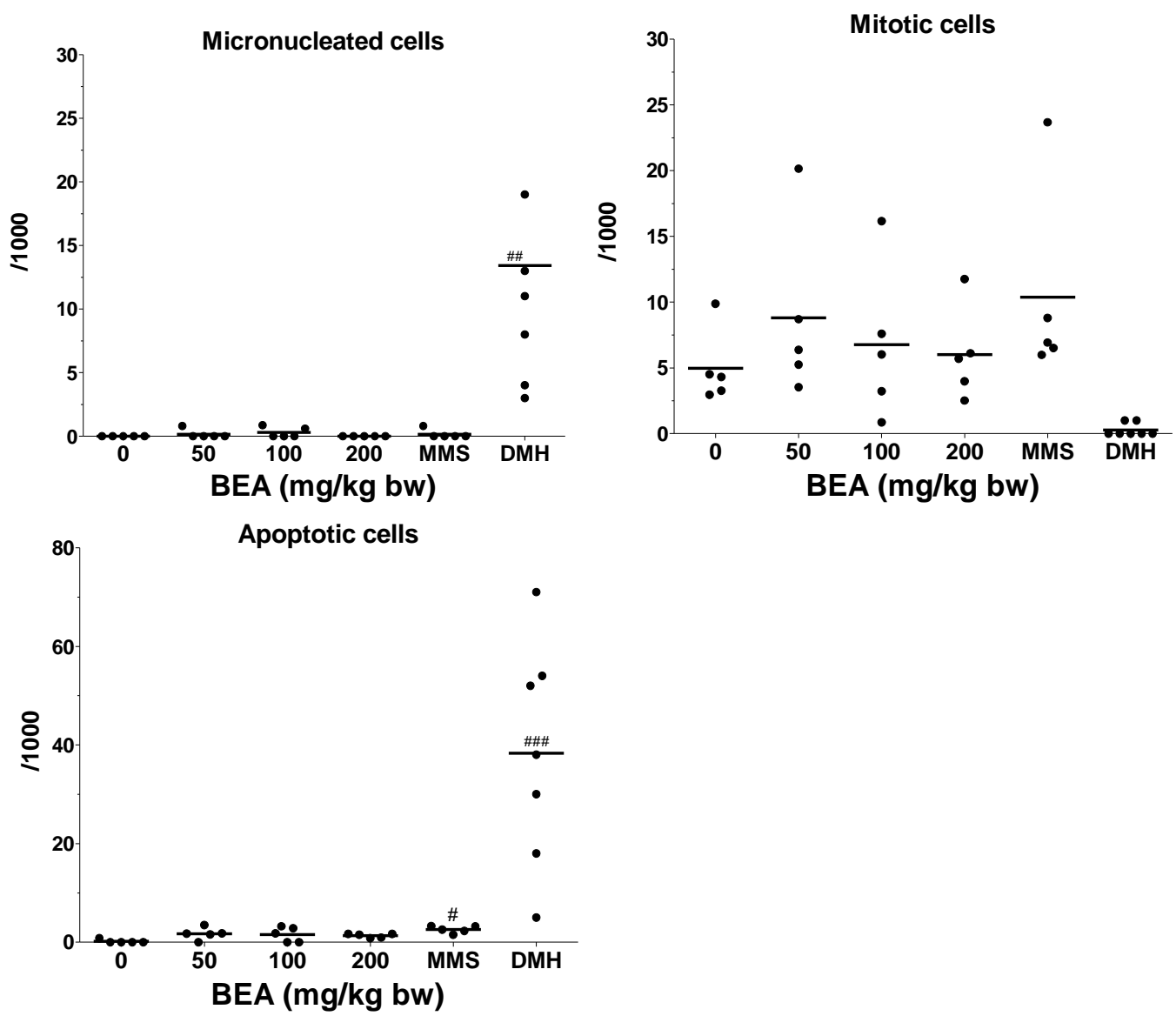

Figure 6: Individual results ( 5 animals/group) obtained in the colon Micronucleus assay after a 3-day oral administration treatment with different doses of BEA (0 - colza oil+DMSO5\%0, 50, 100 and $200 \mathrm{mg} / \mathrm{kg}$ b.w per day). Frequencies of micronucleated, mitotic and apoptotic epithelial colon cells are shown. MMS and DMH (7 animals) were used as positive CTRL. \# $\mathrm{p}<0.05$, \#\# $\mathrm{p}<0.01$, \#\#\# $\mathrm{p} 0.001$.

\subsubsection{BEA - Micronucleus test in bone marrow}

The positive CTRL MMS clearly increased the frequency of MN PCE. No significant induction of MN formation was observed in male mice treated with BEA (Table 8).

Table 8: Bone-marrow Micronucleus formation determined after 2 oral treatments of male mice with different doses of BEA ( 0 - colza oil+DMSO5\%, 50, 100 and $200 \mathrm{mg} / \mathrm{kg} \mathrm{b.w.} \mathrm{per} \mathrm{day)} \mathrm{or}$ with MMS as positive CTRL.

\begin{tabular}{cccc}
\hline Treatment & Dose $(\mathbf{m g} / \mathbf{k g})$ & $\begin{array}{c}\text { \%o MN PCE } \\
(\mathbf{m e a n} \pm \mathbf{S D})\end{array}$ & $\begin{array}{c}\text { PCE/ NCE } \\
(\mathbf{m e a n} \pm \mathbf{S D})\end{array}$ \\
\hline CTRL & Colza oil+ DMSO5\% & $1.48 \pm 0.02$ & $1.14 \pm 0.54$ \\
BEA & 50 & $1.18 \pm 0.28$ & $1.52 \pm 0.58$ \\
& 100 & $1.05 \pm 0.62$ & $1.90 \pm 0.36$ \\
& 200 & $0.87 \pm 0.43$ & $1.44 \pm 0.51$ \\
\hline MMS & 80 & $9.40 \pm 4.64 \# \# \#$ & $1.24 \pm 0.57$ \\
\hline$\# \# \#$ p & & &
\end{tabular}




\subsection{5. $\quad$ ENNB - Clinical observation and toxicity effects}

No mortality, no clinical signs of toxicity and no significant reduction in body weight (Table 9) were present after 2 oral administrations of ENNB. No macroscopic effects on any organs were noticed in any group.

Table 9: Body weight gain at $24 \mathrm{~h}$ in male mice administered with different doses of ENNB (0 colza oil+DMSO10\%, 50, 100 and $200 \mathrm{mg} / \mathrm{kg}$ per day). Results are expressed as mean \pm SD of 5 animals.

\begin{tabular}{llc}
\hline \multirow{2}{*}{ Treatment } & \multirow{2}{*}{ Dose (mg/kg b.w.) } & b.w. gain $(\mathbf{m g})$ \\
\cline { 3 - 3 } & & $\mathbf{2 4 h}$ \\
\hline CTRL & Colza oil+DMSO10\% & $-0.04 \pm 0.55$ \\
ENNB & $\mathbf{5 0}$ & $-0.16 \pm 0.43$ \\
& $\mathbf{1 0 0}$ & $0.36 \pm 1.36$ \\
& $\mathbf{2 0 0}$ & $0.8 \pm 0.78$ \\
\hline
\end{tabular}

In order to know if ENNB induced systemic toxicity effect in male mice after 2 oral gavage, we performed histopathological analysis of liver slides. We observed cytotoxic effect on liver predominantly in the middle dose group with centrilobular swelling and several mitotic cells at mid and high doses (Table 10). These results clearly indicated that ENNB induced cytotoxic effect in liver at 50, 100 and $200 \mathrm{mg} / \mathrm{kg}$ b.w. 
Table 10: Incidence for each histopathological effect on liver of male mice after 2-day oral administration with different doses of ENNB (0 - colza oil+DMSO10\%, 50, 100 and $200 \mathrm{mg} / \mathrm{kg}$ per day).

\begin{tabular}{|c|c|c|c|c|c|c|}
\hline Treatment & $\begin{array}{c}\text { Dose } \\
(\mathrm{mg} / \mathrm{kg} \mathrm{b.w./d})\end{array}$ & Steatosis $^{a}$ & Inflammation $^{a}$ & Clarification $^{a}$ & Necrosis & Mitosis \\
\hline \multirow[t]{5}{*}{ CTRL } & $\begin{array}{l}\text { Colza oil + } \\
\text { DMSO10\% }\end{array}$ & 1 (Microvesicular) & 0 & 0 & 0 & 1 \\
\hline & & 1 (Microvesicular) & + & 0 & 0 & 0 \\
\hline & & 1 (Microsteatosis) & 0 & 0 & 0 & 0 \\
\hline & & 0 & 0 & 0 & 0 & 0 \\
\hline & & 1 (microsteatosis) & 0 & 1 & 0 & 0 \\
\hline \multirow[t]{15}{*}{ ENNB } & 50 & $\begin{array}{c}1 \text { (Macro et } \\
\text { Microvesicular) }\end{array}$ & 0 & 0 & 0 & 2 \\
\hline & & 3 (Microvesicular) & 0 & 0 & 0 & 3 \\
\hline & & 3 (Microvesicular) & 0 & 0 & 0 & 2 \\
\hline & & 3 (Microvesicular) & 0 & 0 & 0 & 0 \\
\hline & & 3 (Microvesicular) & 0 & 1 & 0 & 0 \\
\hline & 100 & $\begin{array}{c}3 \text { (Macro et } \\
\text { Microvesicular) }\end{array}$ & 0 & 0 & 0 & $>10$ \\
\hline & & 2 (Microvesicular) & 0 & 0 & 0 & $>10$ \\
\hline & & 1 (Microvesicular) & 0 & 0 & 0 & 0 \\
\hline & & 1 (Microvesicular) & 1 & 0 & 0 & $>10$ \\
\hline & & 1 (Microvesicular) & 0 & 0 & 0 & 10 \\
\hline & 200 & 2 (Microvesicular) & 0 & 0 & 0 & $>10$ \\
\hline & & 2 (Microvesicular) & 0 & 0 & 0 & 10 \\
\hline & & 2 (Microvesicular) & 0 & 0 & 0 & 4 \\
\hline & & 2 (Microvesicular) & 0 & 0 & 0 & 6 \\
\hline & & 1 (Microvesicular) & 0 & 0 & 0 & 10 \\
\hline
\end{tabular}

${ }^{a}$ five grades between 1 and 5 were used - minimal (1), sligh (2), moderate (3), marked (4) or severe (5).

${ }^{\mathrm{b}}$ Number of mitotic cells $/ \mathrm{mm}^{2}$

\subsubsection{ENNB - Alkaline Comet assay}

\section{First study}

The Comet assay results with ENNB after 2-day oral exposures on mice are presented on Figures 7-8 and Table 11. For all organs, low level of DNA damage was detected in negative CTRL group. The mean of the medians of \%TI of the negative CTRL group ranged from $0.16 \%$ (blood) up to $1.44 \%$ (colon) whereas the positive group treated with $80 \mathrm{mg} / \mathrm{kg}$ b.w. MMS ranged from $8.67 \%$ (duodenum) up to $13.71 \%$ (kidney). The values of the MMS group were significantly increased in all tissues over those of the negative CTRL.

Overall, ENNB treatment did not significantly increase the DNA migration in any organ, except in the kidney exposed to the dose of $50 \mathrm{mg} / \mathrm{kg} \mathrm{b.w.} \mathrm{(Figure} \mathrm{7).}$ 

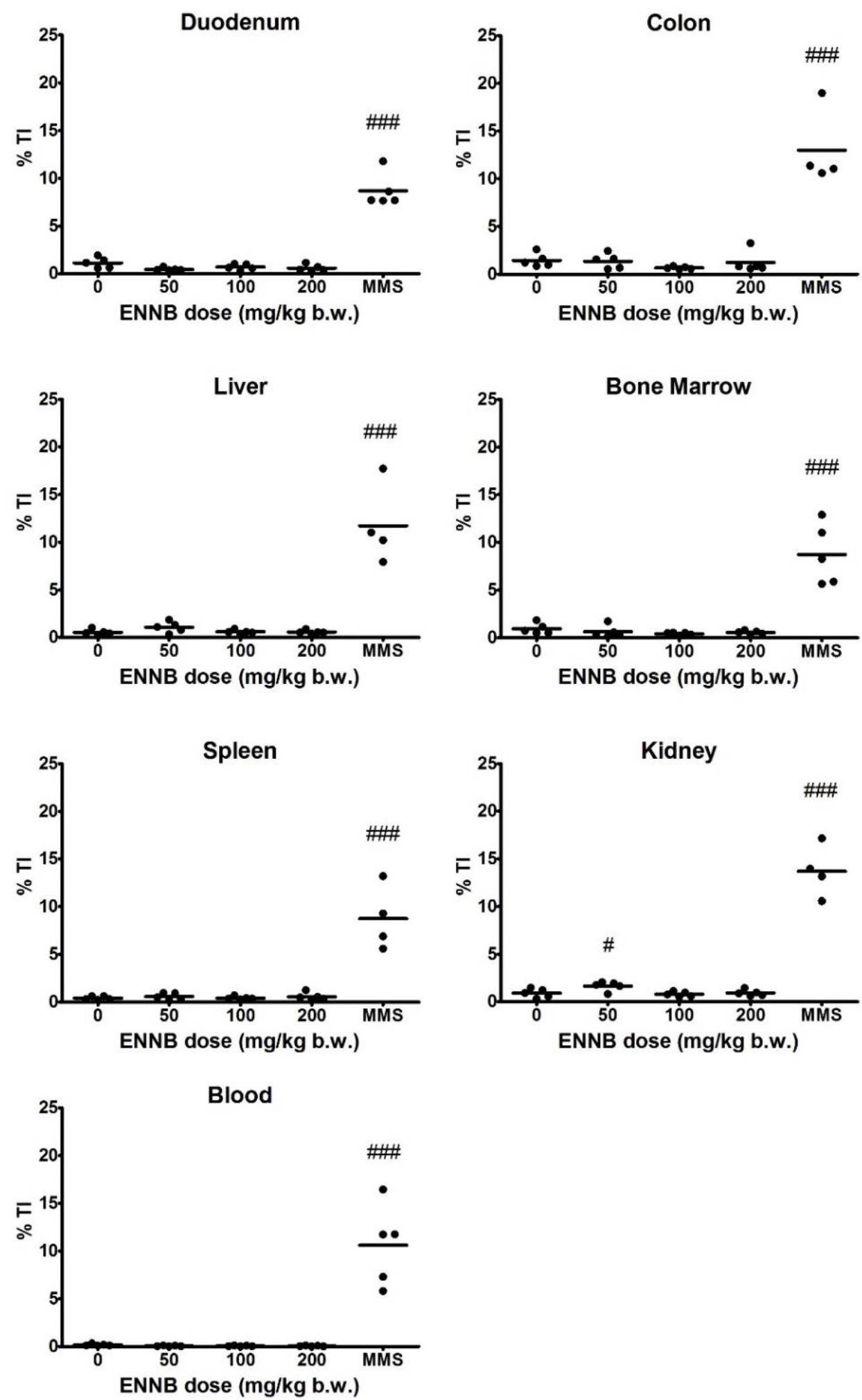

Figure 7: Individual results ( 5 animals/group) obtained in the Comet assay responses in different tissues of male mice after 2-day oral administration with different doses of ENNB (0 - colza oil+DMSO10\%, 50, 100 and $200 \mathrm{mg} / \mathrm{kg}$ per day). DNA damage is expressed as \% of tail DNA intensity. MMS treatment was used for positive CTRL group. Lines indicate mean of medians of percentage tail intensity in each group. \# $p<0.05$; \#\# $p<0.001$. 

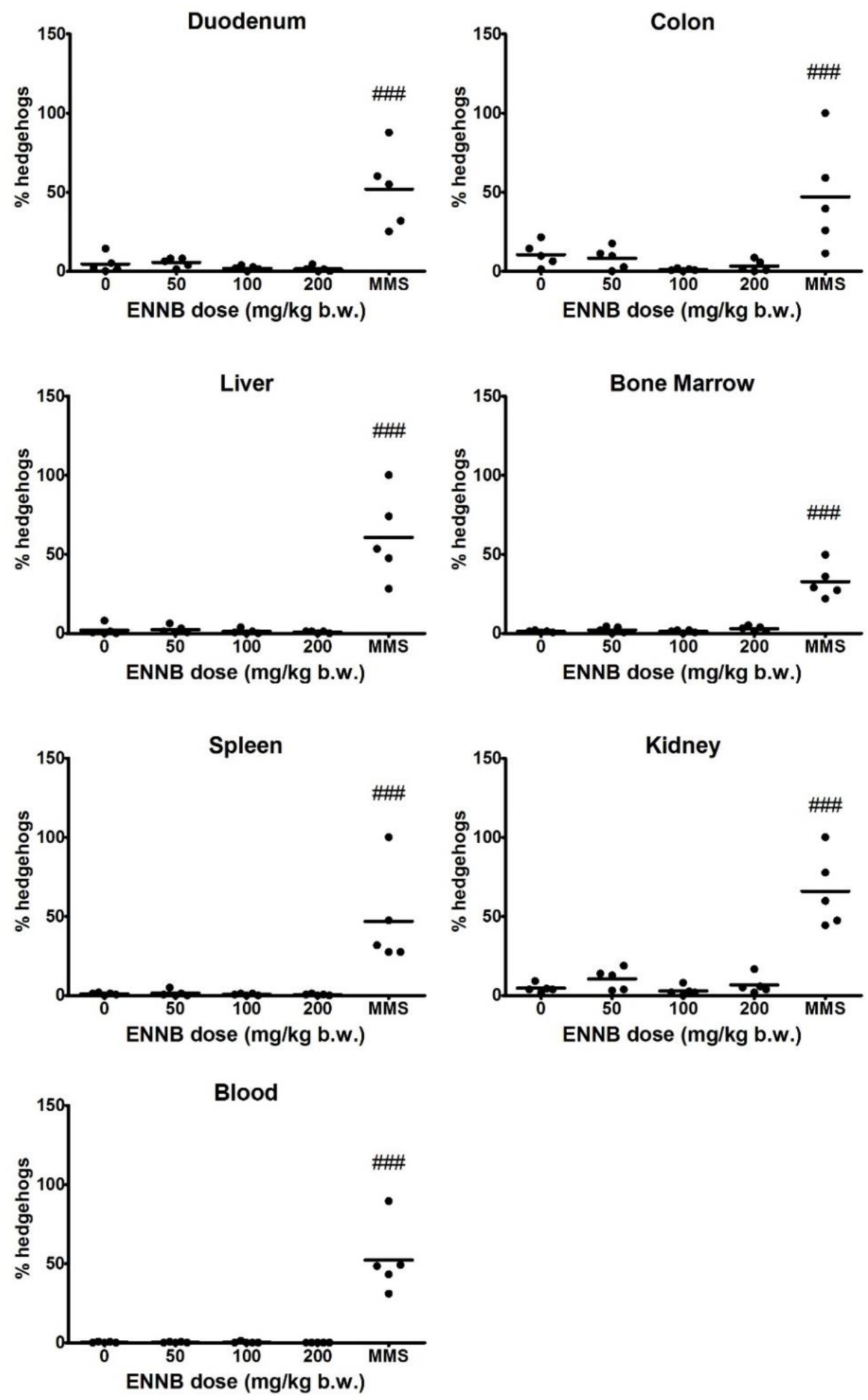

Figure 8: Individual results ( 5 animals/group) for $\%$ of hedgehogs cells obtained by the Comet assay in different tissues of male mice after 2-day oral administration with different doses of ENNB (0 - colza oil+DMSO10\%, 50, 100 and $200 \mathrm{mg} / \mathrm{kg}$ per day). MMS treatment was used for positive CTRL group. Lines indicate mean of $\%$ hedgehogs per groups. \#\#\# $p<$ 0.001 . 
Table 11: Alkaline Comet assay in different organs/tissues of male mice treated after 2-day oral administration with different doses of ENNB (0 - colza oil+DMSO10\%, 50, 100 and $200 \mathrm{mg} / \mathrm{kg}$ per day) or with MMS as positive CTRL. For each experimental group the mean of medians \% DNA in tail and \% hedgehogs are reported.

\begin{tabular}{|c|c|c|c|c|}
\hline Tissues/organs & Treatment & $\begin{array}{c}\text { Dose } \\
\text { (mg/kg b.w./d) }\end{array}$ & $\begin{array}{c}\% \text { TI } \\
( \pm \text { SD) }\end{array}$ & $\begin{array}{c}\text { \% Hedgehogs } \\
( \pm \text { SD) }\end{array}$ \\
\hline \multirow{5}{*}{ Blood } & CTRL & Colza oil+ DMSO10\% & $0.16 \pm 0.10$ & $0.27 \pm 0.56$ \\
\hline & ENNB & 50 & $0.06 \pm 0.04$ & $0.27 \pm 0.55$ \\
\hline & & 100 & $0.06 \pm 0.04 \#$ & $0.27 \pm 0.82$ \\
\hline & & 200 & $0.05 \pm 0.04 \#$ & $0.00 \pm 0.00$ \\
\hline & MMS & 80 & $9.97 \pm 4.20 \# \# \#$ & $50.2 \pm 22.37 \# \# \#$ \\
\hline \multirow{5}{*}{ Bone marrow } & CTRL & Colza oil+ DMSO10\% & $0.95 \pm 0.56$ & $1.19 \pm 1.23$ \\
\hline & ENNB & 50 & $0.62 \pm 0.63$ & $2.22 \pm 3.74$ \\
\hline & & 100 & $0.41 \pm 0.14$ & $1.19 \pm 1.88$ \\
\hline & & 200 & $0.56 \pm 0.18$ & $2.98 \pm 2.52$ \\
\hline & MMS & 80 & $8.73 \pm 3.18 \# \# \#$ & $34.27 \pm 10.45 \# \# \#$ \\
\hline \multirow{5}{*}{ Liver } & CTRL & Colza oil+ DMSO10\% & $0.52 \pm 0.32$ & $2.09 \pm 3.70$ \\
\hline & ENNB & 50 & $1.08 \pm 0.57$ & $2.47 \pm 3.17$ \\
\hline & & 100 & $0.59 \pm 0.19$ & $1.19 \pm 1.88$ \\
\hline & & 200 & $0.57 \pm 0.18$ & $0.79 \pm 1.23$ \\
\hline & MMS & 80 & $11.72 \pm 4.21 \# \# \#$ & $56.86 \pm 29.22 \# \# \#$ \\
\hline \multirow{5}{*}{ Kidney } & CTRL & Colza oil+ DMSO10\% & $0.90 \pm 0.47$ & $4.7 \pm 3.11$ \\
\hline & ENNB & 50 & $1.65 \pm 0.49 \#$ & $10.93 \pm 7.38$ \\
\hline & & 100 & $0.8 \pm 0.26$ & $2.98 \pm 3.01$ \\
\hline & & 200 & $0.92 \pm 0.32$ & $6.95 \pm 6.42$ \\
\hline & MMS & 80 & $13.71 \pm 2.72 \# \# \#$ & $50.08 \pm 24.55 \# \# \#$ \\
\hline \multirow{5}{*}{ Spleen } & CTRL & Colza oil+ DMSO10\% & $0.42 \pm 0.19$ & $1.06 \pm 1.89$ \\
\hline & ENNB & 50 & $0.58 \pm 0.36$ & $1.45 \pm 3.55$ \\
\hline & & 100 & $0.43 \pm 0.16$ & $0.66 \pm 1.20$ \\
\hline & & 200 & $0.56 \pm 0.41$ & $0.53 \pm 0.90$ \\
\hline & MMS & 80 & 8.74 土 3.35\#\#\# & $43.93 \pm 29.69 \# \# \#$ \\
\hline \multirow{5}{*}{ Duodenum } & CTRL & Colza oil+ DMSO10\% & $1.13 \pm 0.56$ & $4.82 \pm 6.31$ \\
\hline & ENNB & 50 & $0.45 \pm 0.15$ & $5.54 \pm 4.82$ \\
\hline & & 100 & $0.69 \pm 0.31$ & $1.96 \pm 2.36$ \\
\hline & & 200 & $0.58 \pm 0.34$ & $1.45 \pm 2.40$ \\
\hline & MMS & 80 & $8.67 \pm 1.77 \# \# \#$ & $54.65 \pm 25.38 \# \# \#$ \\
\hline \multirow{5}{*}{ Colon } & CTRL & Colza oil+ DMSO10\% & $1.44 \pm 0.70$ & $11.14 \pm 8.42$ \\
\hline & ENNB & 50 & $1.36 \pm 0.77$ & $8.65 \pm 7.07$ \\
\hline & & 100 & $0.65 \pm 0.14$ & $0.92 \pm 1.24$ \\
\hline & & 200 & $1.22 \pm 1.13$ & $3.35 \pm 3.68$ \\
\hline & MMS & 80 & $12.99 \pm 4.00 \# \# \#$ & $44.16 \pm 33.20 \# \# \#$ \\
\hline
\end{tabular}

$\# p<0.05 ; \# \#$; $<0.001$

In order to discriminate among necrosis, apoptosis and a possible genotoxic effect of ENNB in the kidney at the lowest dose, a histopathological study was performed followed by a specific quantification of DNA-double strand breaks by $\mathrm{YH} 2 \mathrm{AX}$ staining. No histopathological findings different from the CTRL were observed in the kidney of ENNB treated male mice. Moreover, the $\mathrm{yH} 2 \mathrm{AX}$ staining did not show any significant effect of the mycotoxin or MMS treatment compared to the solvent CTRL (Fig. 9). The slight positive result observed by Comet assay in kidney at the lowest ENNB dose (\%TI values of $1.65 \pm 0.49$ compared to $0.90 \pm 0.47$ of the negative $C T R L$ ) could therefore be explained by a small induction of single DNA strand breaks. 


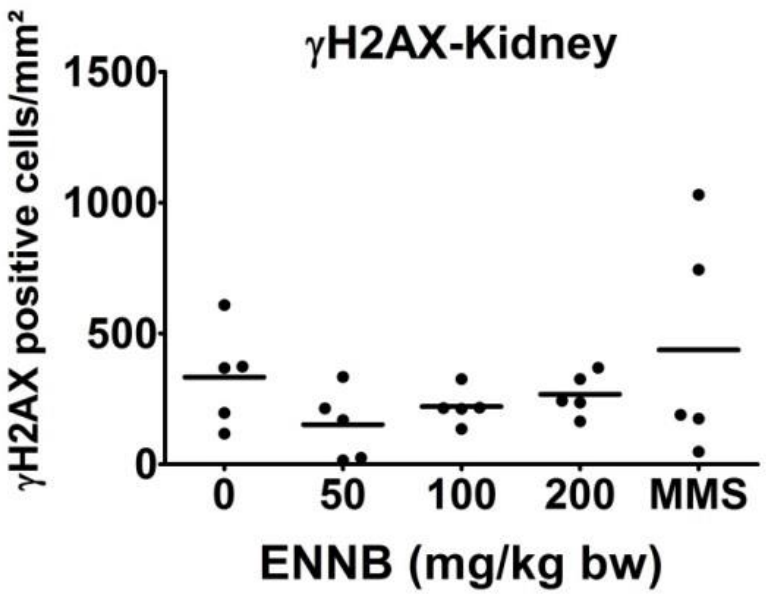

Figure 9: Individual results (5 animals/group) obtained in the $\mathrm{yH} 2 \mathrm{AX}$ staining of kidney slides obtained from male mice after 2-day oral administration with different doses of ENNB (0 colza oil+DMSO10\%, 50, 100 and $200 \mathrm{mg} / \mathrm{kg}$ per day). MMS treatment was used for positive CTRL group. Lines indicate group means of $\mathrm{yH} 2 \mathrm{AX}$-positive cell incidence.

\section{Second study}

In the first study, the electrophoresis condition (voltage $0.37 \mathrm{~V} / \mathrm{cm}$ ) applied was not optimal to reveal a low level of DNA damage. Even if the results of this study are to be considered valid since the positive CTRL values were always statistically increased over negative CTRL values, we decided to carry out a second experiment applying more sensitive conditions. In this second experiment DNA damage was assessed, after 2 oral administrations of ENNB, in two relevant organs, the liver, because it is the most active organ in metabolism of substances and also frequently a target for carcinogenicity and the bone marrow to evaluate a systemic effect.

In this second experiment a dose-dependent increase of DNA migration was observed in both organs, being statistically significant at 100 and $200 \mathrm{mg} / \mathrm{kg} \mathrm{b.w.} \mathrm{in} \mathrm{liver} \mathrm{and} \mathrm{at} 200 \mathrm{mg} / \mathrm{kg}$ in bone marrow (Table 12 and Fig.10). The positive results obtained in these two organs were considered to be sufficient to assume the mycotoxin as a genotoxic substance.

Table 12: Alkaline Comet assay in liver and bone marrow of mice after 2 oral administration with different doses of ENNB ( 0 - colza oil+DMSO10\%, 50, 100 and $200 \mathrm{mg} / \mathrm{kg}$ per day) or with MMS as positive CTRL. For each experimental group the mean of medians \% DNA in tail and \% hedgehogs are reported.

\begin{tabular}{|c|c|c|c|c|}
\hline Tissues/organs & Treatment & $\begin{array}{c}\text { Dose } \\
\text { (mg/kg b.w. day) }\end{array}$ & $\begin{array}{c}\% \text { TI } \\
( \pm \text { SD) }\end{array}$ & $\begin{array}{c}\text { \% Hedgehogs } \\
( \pm \text { SD) }\end{array}$ \\
\hline \multirow{5}{*}{ Liver } & CTRL & Colza oil+ DMSO10\% & $0.46 \pm 0.22$ & $2.60 \pm 0.89$ \\
\hline & ENNB & 50 & $1.76 \pm 1.00$ & $3.00 \pm 1.58$ \\
\hline & & 100 & $2.97 \pm 0.70 \#$ & $2.80 \pm 1.92$ \\
\hline & & 200 & $5.27 \pm 2.49 \# \# \#$ & $3.60 \pm 2.07$ \\
\hline & MMS & 80 & 13.79 土 2.94\#\#\# & 8.40 土 2.07\#\#\# \\
\hline \multirow{5}{*}{ Bone marrow } & CTRL & Colza oil+ DMSO10\% & $0.47 \pm 0.13$ & $0.80 \pm 0.84$ \\
\hline & ENNB & 50 & $1.16 \pm 0.54$ & $0.60 \pm 0.89$ \\
\hline & & 100 & $1.57 \pm 0.44$ & $0.80 \pm 0.84$ \\
\hline & & 200 & $2.60 \pm 1.34 \# \#$ & $0.60 \pm 0.89$ \\
\hline & MMS & 80 & $2.99 \pm 1.05 \# \#$ & $4.20 \pm 1.48 \# \#$ \\
\hline
\end{tabular}

$\# p<0.05 ; \# \# p<0.01 ; \# \# \# p<0.001$ 

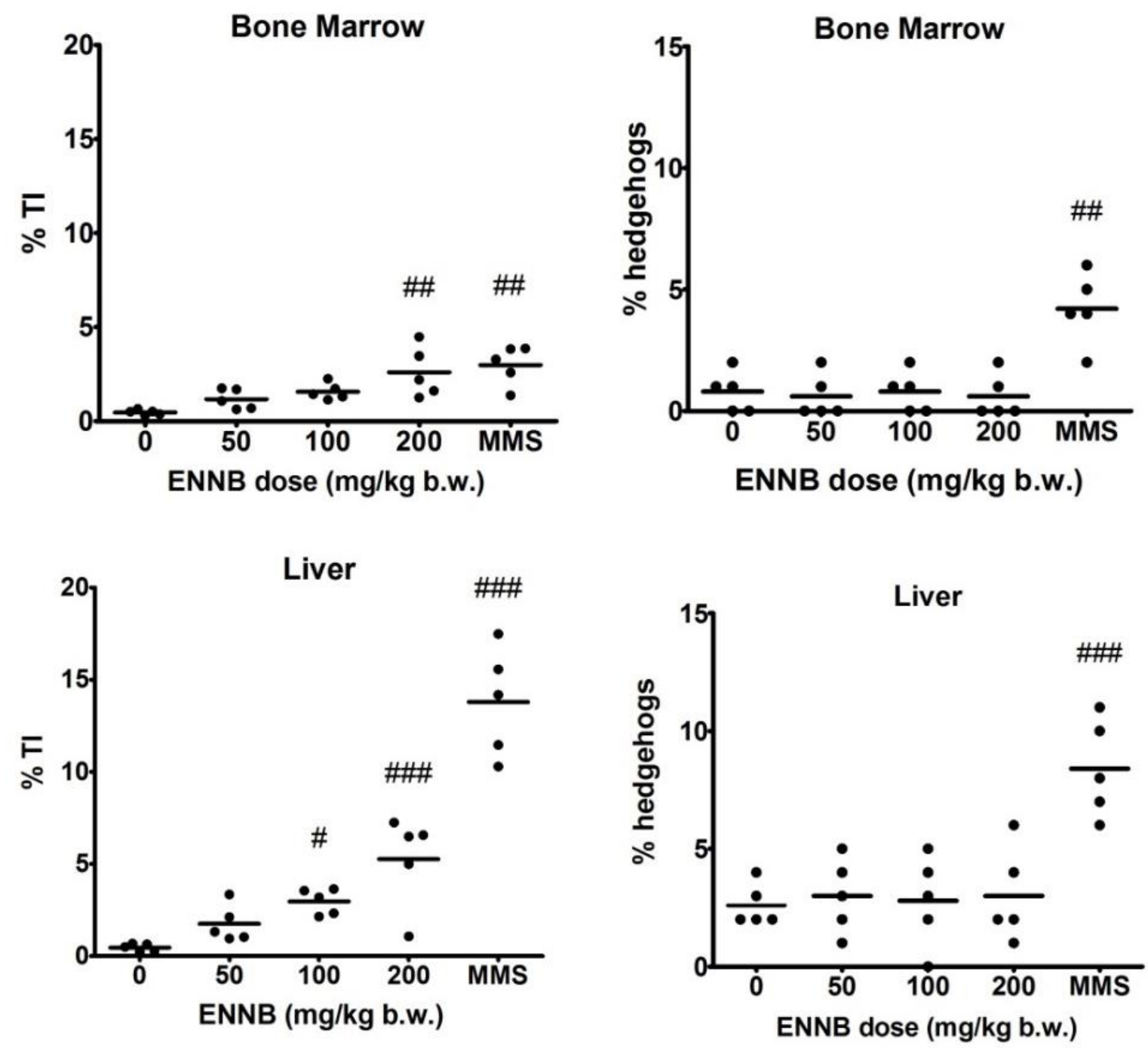

Figure 10: Individual results (5 animals/group) obtained by the Comet assay in different tissues of male mice after 2 oral treatments with different doses of ENNB (0 - colza oil+DMSO10\%, 50, 100 and $200 \mathrm{mg} / \mathrm{kg}$ per day). DNA damage is expressed as \% of tail DNA intensity; \% of hedgehogs is also shown. MMS treatment was used for positive CTRL group. Lines indicate mean of medians of percentage tail intensity and hedgehogs. \# $p<$ 0.05; \#\# $\mathrm{p}<0.01$; \#\# $\mathrm{p}<0.001$.

In order to discriminate between necrosis and/or apoptosis and a possible genotoxic effect of ENNB in the liver, a histopathological study was performed. Whereas microvesicular steatosis was observed in all mice due to colza oil, ENNB increased lobular inflammation without a clear dose-effect relationship. The marked effect of ENNB in the liver is the dose-related increases of mitotic cells without effect on necrosis or apoptosis. These results indicated that ENNB induced genotoxic effect in liver without induced apoptosis or necrosis (Table 13). 
Table 13: Histopathological analysis of liver after 2 oral treatments with ENNB

\begin{tabular}{|c|c|c|c|c|c|c|}
\hline Treatment & $\begin{array}{c}\text { Dose } \\
\text { (mg/kg.b.w./d) }\end{array}$ & Steatosis $^{\mathrm{a}}$ & $\begin{array}{c}\text { Steatosis } \\
\text { type }\end{array}$ & $\begin{array}{c}\text { Lobular } \\
\text { inflammation }\end{array}$ & $\begin{array}{c}\text { Necrosis } \\
\text { /apoptosis }\end{array}$ & Mitosis $^{c}$ \\
\hline \multirow[t]{5}{*}{ CTRL } & \multirow[t]{5}{*}{$\begin{array}{l}\text { Colza oil + } \\
\text { DMSO10\% }\end{array}$} & 3 & microvesicular & (1) & $=$ & 0.11 \\
\hline & & 3 & microvesicular & 0 & 0 & 0.05 \\
\hline & & 3 & microvesicular & 0 & 0 & 0.13 \\
\hline & & 3 & microvesicular & 1 & 0 & 2.57 \\
\hline & & 3 & microvesicular & 0 & 0 & 0.14 \\
\hline \multirow[t]{15}{*}{ ENNB } & \multirow[t]{5}{*}{50} & 3 & microvesicular & 0 & 0 & 0.38 \\
\hline & & 3 & microvesicular & 1 & 0 & 0.31 \\
\hline & & 2 & microvesicular & 1 & 0 & 0.3 \\
\hline & & 0 & - & 0 & 0 & 0.14 \\
\hline & & 1 & microvesicular & 3 & 0 & 3.54 \\
\hline & \multirow[t]{5}{*}{100} & 3 & microvesicular & 2 & 0 & 0.55 \\
\hline & & 3 & microvesicular & 2 & 0 & 3.26 \\
\hline & & 3 & microvesicular & 1 & 0 & 2.79 \\
\hline & & 0 & - & 0 & 0 & 0.04 \\
\hline & & 3 & microvesicular & 0 & 0 & 0.35 \\
\hline & \multirow[t]{5}{*}{200} & 1 & microvesicular & 1 & 0 & 4.76 \\
\hline & & 1 & microvesicular & 1 & 0 & 5.00 \\
\hline & & 1 & microvesicular & 2 & 0 & 3.54 \\
\hline & & 0 & - & 1 & 0 & 9.38 \\
\hline & & 0 & - & 1 & 0 & 3.97 \\
\hline
\end{tabular}

\subsubsection{ENNB - Micronucleus tests in colon}

ENNB did not increase micronucleated, mitotic or apoptotic cells in colon after 2 oral administrations between 50 and $200 \mathrm{mg} / \mathrm{kg}$ b.w. (Fig. 11). The clastogen MMS did not increase MN formation in colon cells, but significantly increased apoptotic cells. DMH clearly increased micronucleated and apoptotic cells in colon. 

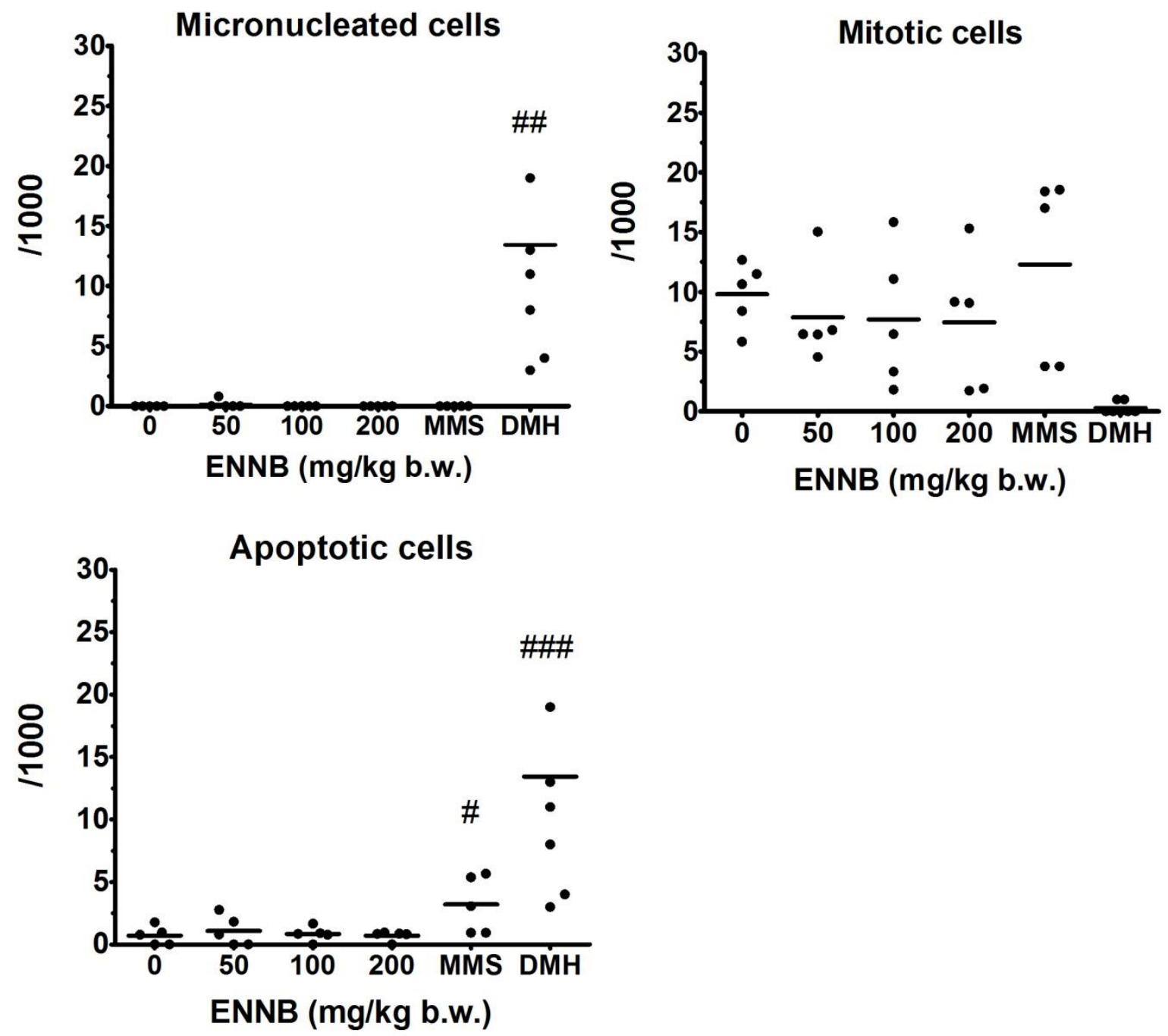

Figure 11: Individual results (5 animals/group) obtained in the colon Micronucleus assay after 2 oral treatments of male mice with different doses of ENNB (0 - colza oil+DMSO10\%, 50, 100 and $200 \mathrm{mg} / \mathrm{kg}$ per day). Frequencies of micronucleated, mitotic and apoptotic epithelial colon cells are reported. MMS and DMH (6 animals) were used as positive CTRL. \# $\mathrm{p}<0.05$; \# $\mathrm{p}<0.01$; \#\# $\mathrm{p}<0.001$.

\subsection{8. $\quad$ ENNB - Micronucleus test in bone marrow}

The positive CTRL MMS clearly increased the frequency of MN PCE (Table14). No significant induction of MN was observed in male mice treated with ENNB (Table 14). 
Table 14: Bone-marrow Micronucleus formation determined in male mice after 2 oral treatments with different doses of ENNB (0 - colza oil+DMSO10\%, 50, 100 and $200 \mathrm{mg} / \mathrm{kg}$ per day) or MMS as positive CTRL.

\begin{tabular}{cccc}
\hline Treatment & $\begin{array}{c}\text { Dose } \\
\text { (mg/kg b.w./d) }\end{array}$ & $\begin{array}{c}\text { \%o MN PCE } \\
\text { (mean } \pm \text { SD) }\end{array}$ & $\begin{array}{c}\text { PCE/NCE } \\
\text { (mean } \pm \text { SD) }\end{array}$ \\
\hline CTRL & Colza oil+ DMSO10\% & $0.59 \pm 0.64$ & $1.10 \pm 0.19$ \\
ENNB & 50 & $0.20 \pm 0.27$ & $0.94 \pm 0.13$ \\
& 100 & $0.59 \pm 0.53$ & $0.90 \pm 0.42$ \\
& 200 & $0.30 \pm 0.27$ & $0.93 \pm 0.19$ \\
\hline MMS & 80 & $9.40 \pm 4.64 \# \# \#$ & $1.24 \pm 0.57$ \\
\hline \#\# p $<0.001$ & & &
\end{tabular}

\subsection{In vitro genotoxicity studies with BEA and ENNB}

To further investigate the genotoxicity and determine the mode of action of the toxins, a series of in vitro experiments, requiring a smaller amount of toxins with respect to in vivo testing, was performed. In particular, the $\mathrm{MN}$ test and the multiparametric $\mathrm{\gamma H} 2 \mathrm{AX}$ and $\mathrm{pH} 3$ test were applied. The $\mathrm{MN}$ test, detecting both aneugens and clastogens, provides a comprehensive basis for the assessment of chromosome damaging potential (OECD $\left.\mathrm{N}^{\circ} 487^{1}\right)$. The phosphorylation of histone $\mathrm{H} 2 \mathrm{AX}$ in the serine 139 (named $\mathrm{yH} 2 \mathrm{AX}$ ) visualizes DNA double strand break repair foci allowing the identification of clastogenic compounds (Rogakou et al., 1998). The phosphorylation of histone $\mathrm{H} 3$ at serine 10 (named $\mathrm{pH} 3$ ) identifies mitotic cells that increase after treatment with aneugenic compounds (Bryce et al., 2017; Khoury et al., 2016). The integration of these tests allows the assessment of the genotoxic potential of the tested toxin and to investigate its mode of action.

\subsubsection{In vitro Micronucleus test in human TK6 cells}

In the in vitro $M N$ test we found that BEA and ENNB failed to induce MN formation whatever the treatment conditions, with or without S9 mix. BEA was less toxic in the presence of S9 mix with a cytostasis of $16.7 \%$ and $100 \%$ with and without S9 mix, respectively, at $10 \mu \mathrm{M}$. On the contrary, the presence of metabolic S9 mix did not affect the cytotoxicity of ENNB in TK6 cells. After a 24h treatment, BEA induced high cytotoxicity, with a cytostasis of $80 \%$ at $5 \mu \mathrm{M}$. ENNB was also highly toxic after $24 \mathrm{~h}$ treatment with cytostasis over $80 \%$ from the concentration of $2.5 \mu \mathrm{M}$. The positive CTRLS MMS without S9 and B[a]P with S9 significantly increased MN formation (Table 15). Both positive controls induced micronuclei while no effect was observed after BEA or ENNB exposure.

\footnotetext{
${ }^{1}$ OECD, Test No. 487, 2010: In Vitro Mammalian Cell Micronucleus Test, OECD Publishing, Paris. 
Table 15: In vitro Micronucleus assays in TK6 cells after 3 hours exposure to BEA and ENNB without and with S9 mix and after 24 hours exposure without S9 mix.

\begin{tabular}{|c|c|c|c|c|c|}
\hline S9 & $\begin{array}{c}\text { Time of } \\
\text { exposure }(h)\end{array}$ & Compound & $\mu \mathrm{M}$ & MN (\%) & $\begin{array}{c}\text { Cytostasis } \\
\text { (100-RPD) } \\
(\%)\end{array}$ \\
\hline \multirow[t]{12}{*}{ Without } & 3 & BEA & 0 & 2.5 & 0 \\
\hline & & & 0.625 & 2.5 & 36.7 \\
\hline & & & 1.25 & 2.5 & 20.3 \\
\hline & & & 2.5 & 2 & 34.9 \\
\hline & & & 5 & 5 & 41.2 \\
\hline & & & 10 & TOX & 100 \\
\hline & & ENNB & 0.625 & 1 & 14.0 \\
\hline & & & 1.25 & 1.5 & 7.3 \\
\hline & & & 2.5 & 1 & 30.4 \\
\hline & & & 5 & 2.5 & 19.5 \\
\hline & & & 10 & 1 & 19.8 \\
\hline & & MMS & 45 & 14.5\#\#\# & 37.1 \\
\hline \multirow[t]{12}{*}{ With } & 3 & BEA & 0 & 2 & 0 \\
\hline & & & 0.625 & 2 & 8.2 \\
\hline & & & 1.25 & 1.5 & 0 \\
\hline & & & 2.5 & 1.0 & 0 \\
\hline & & & 5 & 2.49 & 31.9 \\
\hline & & & 10 & 3.49 & 16.7 \\
\hline & & ENNB & 0.625 & 2.5 & 0 \\
\hline & & & 1.25 & 4.99 & 0 \\
\hline & & & 2.5 & 4.49 & 2.6 \\
\hline & & & 5 & 3.5 & 12.6 \\
\hline & & & 10 & 2.5 & 26.7 \\
\hline & & $B[a] P$ & 8 & 76.77\#\#\# & 24.0 \\
\hline \multirow[t]{12}{*}{ Without } & 24 & BEA & 0 & 2.0 & 0 \\
\hline & & & 0.312 & 1.5 & 6.1 \\
\hline & & & 0.625 & 3.99 & 11.6 \\
\hline & & & 1.25 & 3.0 & 27.2 \\
\hline & & & 2.5 & 2.0 & 79.4 \\
\hline & & & 5 & 2.5 & 82.1 \\
\hline & & ENNB & 0.625 & 7.29 & 3.2 \\
\hline & & & 1.25 & 1.5 & 5.3 \\
\hline & & & 2.5 & 5.49 & 88.0 \\
\hline & & & 5 & 3.5 & 94.2 \\
\hline & & & 10 & 3.0 & 106.3 \\
\hline & & MMS & 45 & 12.99\#\# & 20.9 \\
\hline
\end{tabular}

$\# \# p<0.01 ; \# \# \# p<0.001$. RPD = Relative population doubling.

\subsubsection{In vitro Micronucleus test in human undifferentiated HepaRG cells}

The cytokinesis-blocked MN assay on HepaRG cells showed that BEA induced a dose-dependent increase in the frequency of BiNucleated MicroNucleated (BNMN) cells after $4 \mathrm{~h}$ of treatment, with $5.21 \%$ of BNMN at $6.3 \mu \mathrm{M}$ of BEA compared to $2.87 \%$ for negative CTRL. At the same time, BEA decreased cell proliferation by increasing cytostasis to $30 \%$ at $6.3 \mu \mathrm{M}$ (Table 16). ENNB failed to increase both the frequency of BNMN and cytostasis between 0.8 and $25 \mu \mathrm{M}$ after $4 \mathrm{~h}$ of treatment. The positive CTRL, MMS, clearly increased the frequency of BNMN (Table 16). 
Table 16: In vitro Micronucleus assays in human HepaRG cells after $4 \mathrm{~h}$ exposure to BEA and ENNB without S9 mix

\begin{tabular}{lccccc}
\hline Treatment & $\boldsymbol{\mu M}$ & $\mathbf{B N}$ & BNMN & \%BNMN & Cytostasis \\
\hline BEA & 0 & 1045 & 30 & 2.87 & 0 \\
& 0.8 & 1001 & 26 & 2.60 & 14 \\
& 1.6 & 1004 & 37 & 3.69 & 0 \\
& 3.1 & 1011 & 38 & 3.76 & 10 \\
& 6.3 & 1037 & 54 & $5.21 \# \#$ & 30 \\
\hline ENNB & 12.5 & Toxic & - & - & - \\
& 0 & 1045 & 30 & 2.87 & 20 \\
& 0.8 & 1087 & 40 & 3.7 & 11 \\
& 1.6 & 1059 & 23 & 2.2 & 0 \\
& 3.1 & 1000 & 38 & 3.8 & 0 \\
& 6.3 & 1000 & 37 & 3.7 & 1 \\
& 12.5 & 1017 & 29 & 2.9 & 44 \\
\hline & 25.0 & 1111 & 32 & 2.9 & \\
\hline
\end{tabular}

\#\# p <0.01; \#\#\#\# p < 0.0001. MMS was used as positive CTRL $(60 \mu \mathrm{g} / \mathrm{ml})$.

\subsubsection{Results of the multiparametric genotoxicity tests with BEA and ENNB on proliferative HepaRG cells}

An in vitro multiplexed high-content screening assay ( $\mathrm{\gamma H} 2 \mathrm{AX}$ and $\mathrm{pH} 3$ ) to distinguish genotoxicants from nongenotoxicants was used to study the genotoxicity potential of BEA and ENNB in vitro on HepaRG cells (Bernacki et al., 2016).

The clastogenic compound MMS highly increased $\mathrm{yH} 2 \mathrm{AX}$ but not histone $\mathrm{H} 3$ phosphorylation, indicating DNA damage induction; on the other hand, the aneugenic compound Colchicine (COL), highly increased $\mathrm{pH} 3$ positive cells indicating mitotic blockage (Figs. 12 and 13).

BEA decreased cells number compared to CTRL, starting from the concentration of $0.39 \mu \mathrm{M}$, and being around $25 \%$ at $0.78 \mu \mathrm{M}$. An increase of $\mathrm{yH} 2 \mathrm{AX}$ and $\mathrm{pH} 3$ were observed with BEA but only at high cytotoxic concentrations over $0.39 \mu \mathrm{M}$.

ENNB showed high effect on cellular proliferation at the lowest concentration tested, with a parallel induction of $\mathrm{yH} 2 \mathrm{AX}$ that remains at the same level until $0.78 \mu \mathrm{M}$ and increased highly at higher concentrations. The cytotoxicity of ENNB did not further increase with the dose until $1.56 \mu \mathrm{M}$ while it increased at higher concentrations (Fig. 12). We also observed a decrease of pH3 positive cells induced by ENNB (Figs. 12 and 13). 

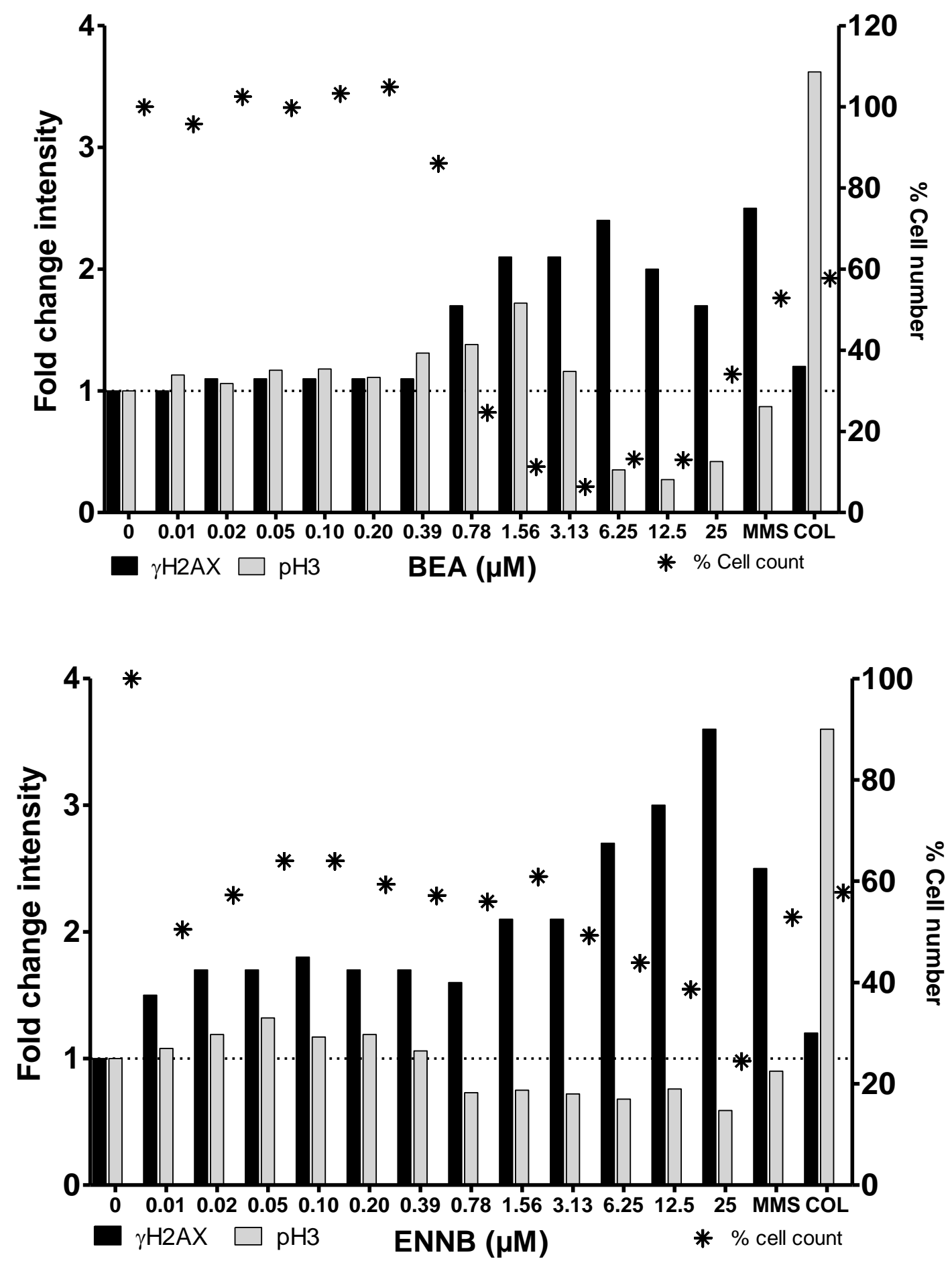

Figure 12: $\quad \mathrm{yH} 2 \mathrm{AX}$ and $\mathrm{pH} 3$ response expressed as fold change with respect to CTRL value (dashed line) (left axe) and percentage of cells with respect to CTRL (right axe) in HepaRG cells after 24h-exposure to BEA (up) or ENNB (bottom). MMS was used as $\mathrm{YH} 2 \mathrm{AX}$ positive CTRL, and COL was used as PH3 positive CTRL. 


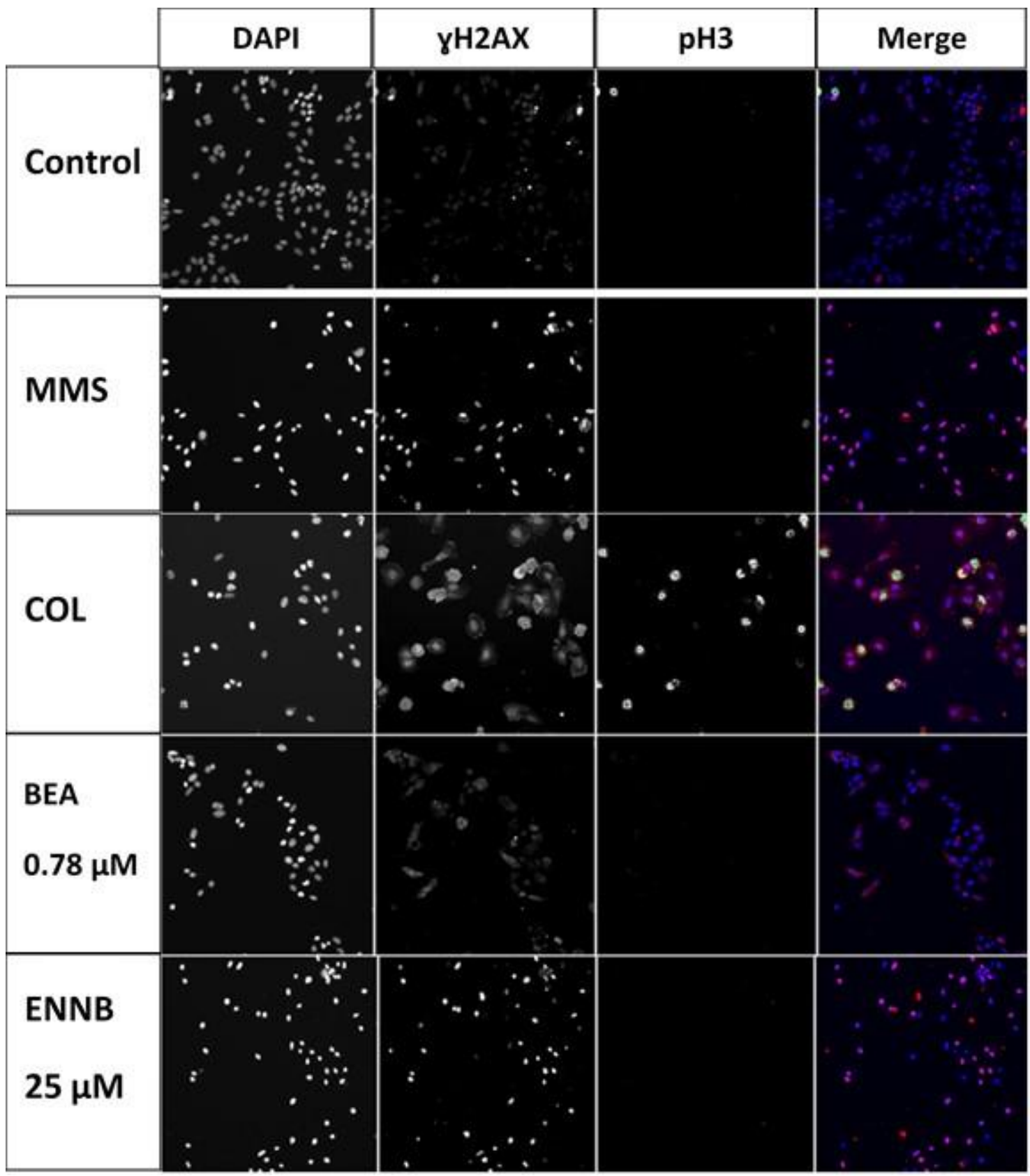

Figure 13: Representative images of HepaRG cells treated for $24 \mathrm{~h}$ with cytotoxic doses of micotoxins. Cells were labeled with DAPI and antibodies against $\mathrm{yH} 2 \mathrm{AX}$ and $\mathrm{pH} 3$ and captured with an Arrayscan VTi. In the right column, the merge of the three fluorescences are reported: Blue (DAPI), red $(\mathrm{\gamma H} 2 \mathrm{AX})$ and green $(\mathrm{pH} 3)$. MMS and $\mathrm{COL}$ were used as positive CTRL for $\mathrm{YH} 2 \mathrm{AX}$ and $\mathrm{pH} 3$ respectively.

\subsection{Repeated dose oral toxicity study of BEA and ENNB}

For the repeated-dose study, BEA has been dissolved in olive oil and DMSO1.5\% (see Solubility Protocol). The BEA experiment has been paired with a CTRL group treated with olive oil only (negative CTRL). Concerns have arisen on the potential toxic effects of DMSO that could have had an impact on BEA results. Indeed, potential DMSO effect would have been evident in all dose groups. Since this was not the case, this issue should not be critical for result interpretation, in particular for effects driving the study NOAEL definition. 


\subsubsection{BEA - General toxicity}

A total of 8 mice died during the course of the study from the following groups/sex (M or F): CTRL olive oil: 1 male and 2 females; BEA0.1: 1 male and one female; BEA1: 2 males and one female. The death was due to gavage procedure and the histopathological evaluation was not performed.

\section{Body Weight}

The body weight of female and male mice treated with BEA at the three different doses increased during the treatment period with no significant alteration induced by BEA (Fig. 14A).
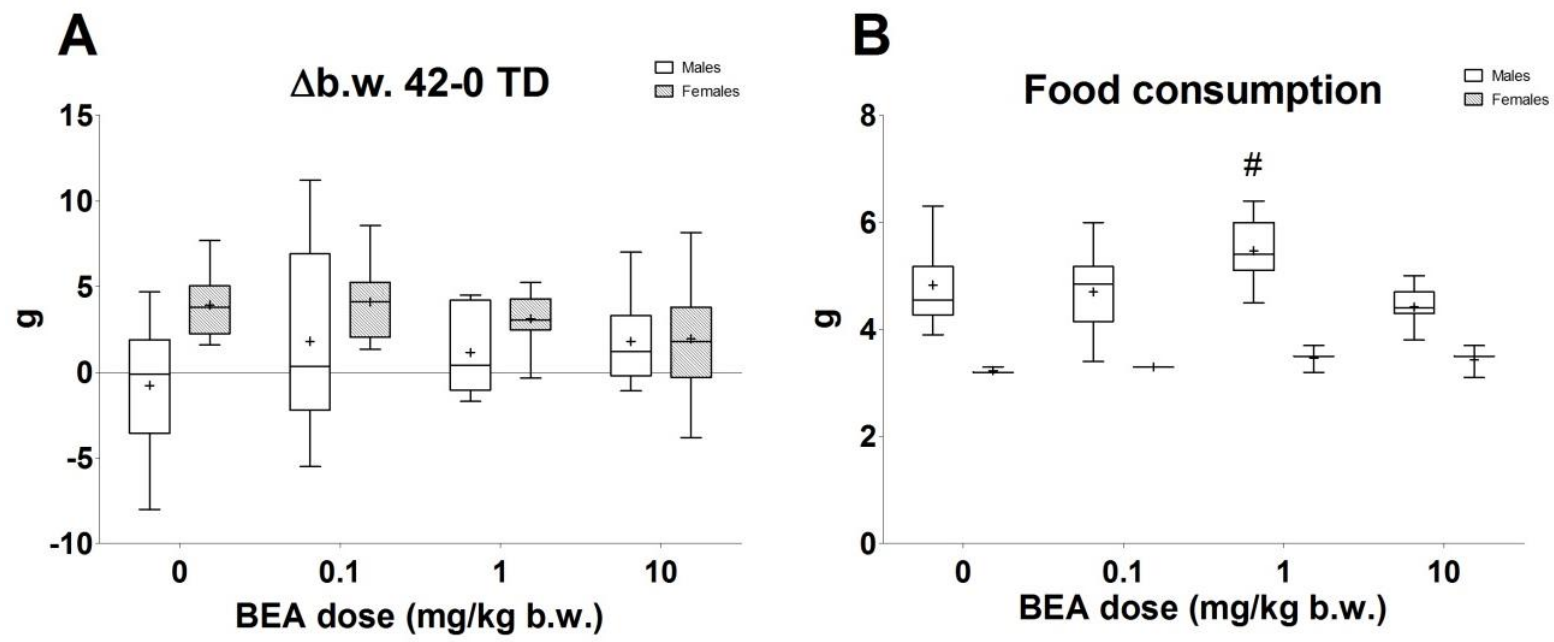

Figure 14: Mean difference in body weight $(A)$ between the end of the treatment period (treatment day TD42) and the beginning of the treatment (TD0) and mean food consumption (B) during the treatment period of male and female mice orally treated for 42 days with different doses of BEA ( 0 - olive oil; $0.1,1$ and $10 \mathrm{mg} / \mathrm{kg}$ per day). Levels are reported as interquartile box plots, with whiskers delimiting the 10th and 90th percentiles. "+" indicates the mean value for each experimental group; \# $p<0.05$.

\section{Food consumption}

The average food consumption of female mice was not significantly different among groups. Conversely, significantly higher food consumption in male mice treated with BEA1 was observed (Fig. 14B).

\section{Organ weight}

Absolute and relative organ weights were summarized in Table 17.

Relative liver weight was significantly decreased by BEA10 treatment in males (Fig. 15B).

Spleen weight was not affected either as absolute or relative value, both in female and male mice (Fig. 15C-D).

Absolute and relative MLN weights were significantly decreased in males treated with BEA1 (Fig. 15EF). No alterations were seen in female mice. 
Table 17: Absolute and relative organ weights of male and female mice orally treated for 42 days with different doses of BEA $(0-$ olive oil; $0.1,1$ and 10 $\mathrm{mg} / \mathrm{kg}$ per day).

\begin{tabular}{|c|c|c|c|c|c|c|c|c|c|}
\hline \multirow[b]{2}{*}{ ORGAN } & & \multicolumn{4}{|c|}{ Male mice } & \multicolumn{4}{|c|}{ Female mice } \\
\hline & & Olive oil & BEA0.1 & BEA1 & BEA10 & Olive oil & BEA0.1 & BEA1 & BEA10 \\
\hline \multirow{4}{*}{ LIVER } & & mean $\pm S D$ & mean $\pm S D$ & mean $\pm S D$ & mean $\pm S D$ & mean $\pm S D$ & mean $\pm S D$ & mean $\pm S D$ & mean $\pm S D$ \\
\hline & $N$ of mice examined & 10 & 10 & 9 & 11 & 9 & 10 & 10 & 11 \\
\hline & Absolute weight (g) & $2.06 \pm 0.28$ & $1.94 \pm 0.23$ & $1.79 \pm 0.33$ & $1.76 \pm 0.26$ & $1.44 \pm 0.19$ & $1.25 \pm 0.21$ & $1.32 \pm 0.24$ & $1.55 \pm 0.16$ \\
\hline & Relative weight $(x$ 100) & $5,38 \pm 0,67$ & $4,76 \pm 0,47$ & $4,76 \pm 0,62$ & $4,50 \pm 0,74 \#$ & $4.52 \pm 0.50$ & $4.14 \pm 0.68$ & $4.59 \pm 0.49$ & $5.00 \pm 0.60$ \\
\hline \multirow[t]{3}{*}{ SPLEEN } & $N$ of mice examined & 10 & 10 & 9 & 11 & 9 & 10 & 10 & 11 \\
\hline & Absolute weight (g) & $0,13 \pm 0,05$ & $0,10 \pm 0,02$ & $0,09 \pm 0,03$ & $0,10 \pm 0,02$ & $0,14 \pm 0,04$ & $0,12 \pm 0,02$ & $0,12 \pm 0,06$ & $0,12 \pm 0,02$ \\
\hline & Relative weight $(x$ 100) & $0,34 \pm 0,16$ & $0,25 \pm 0,05$ & $0,25 \pm 0,08$ & $0,25 \pm 0,04$ & $0,43 \pm 0,13$ & $0,40 \pm 0,08$ & $0,44 \pm 0,23$ & $0,38 \pm 0,07$ \\
\hline \multirow[t]{3}{*}{ MLN } & $N$ of mice examined & 10 & 10 & 9 & 11 & 9 & 10 & 10 & 11 \\
\hline & Absolute weight (g) & $0,03 \pm 0,02$ & $0,02 \pm 0,01$ & $0,01 \pm 0,01 \# \#$ & $0,03 \pm 0,03$ & $0,02 \pm 0,01$ & $0,03 \pm 0,02$ & $0,01 \pm 0,01$ & $0,02 \pm 0,01$ \\
\hline & Relative weight $(x$ 100) & $0,08 \pm 0,04$ & $0,05 \pm 0,04$ & $0,03 \pm 0,02 \# \#$ & $0,07 \pm 0,07$ & $0,07 \pm 0,02$ & $0,10 \pm 0,07$ & $0,04 \pm 0,02$ & $0,06 \pm 0,03$ \\
\hline \multirow[t]{3}{*}{ KIDNEYS } & $N$ of mice examined & 10 & 10 & 9 & 11 & 9 & 10 & 10 & 11 \\
\hline & Absolute weight (g) & $0,68 \pm 0,05$ & $0,62 \pm 0,06$ & $0,55 \pm 0,16 \# \#$ & $0,58 \pm 0,06 \# \#$ & $0,41 \pm 0,04$ & $0,37 \pm 0,04$ & $0,38 \pm 0,05$ & $0,38 \pm 0,02$ \\
\hline & Relative weight $(x$ 100) & $1.78 \pm 0.27$ & $1.51 \pm 0.11 \#$ & $1.46 \pm 0.41$ & $1.48 \pm 0.18 \# \#$ & $1.28 \pm 0.13$ & $1.23 \pm 0.10$ & $1.33 \pm 0.16$ & $1.22 \pm 0.10$ \\
\hline \multirow[t]{3}{*}{ ADRENALS } & $N$ of mice examined & 10 & 10 & 9 & 11 & 9 & 10 & 10 & 11 \\
\hline & Absolute weight (g) & $0,02 \pm 0,01$ & $0,03 \pm 0,01$ & $0,01 \pm 0,01$ & $0,02 \pm 0,01$ & $0,02 \pm 0,01$ & $0,02 \pm 0,01$ & $0,01 \pm 0,01$ & $0,02 \pm 0,01$ \\
\hline & Relative weight $(x$ 100) & $0,06 \pm 0,02$ & $0,07 \pm 0,02$ & $0,04 \pm 0,02$ & $0,06 \pm 0,02$ & $0,05 \pm 0,02$ & $0,05 \pm 0,02$ & $0,05 \pm 0,01$ & $0,06 \pm 0,02$ \\
\hline \multirow[t]{3}{*}{ THYMUS } & $N$ of mice examined & 9 & 10 & 10 & 11 & 9 & 10 & 10 & 11 \\
\hline & Absolute weight (g) & $0,06 \pm 0,02$ & $0,07 \pm 0,03$ & $0,03 \pm 0,02$ & $0,05 \pm 0,01$ & $0,05 \pm 0,01$ & $0,06 \pm 0,02$ & $0,03 \pm 0,01$ & $0,04 \pm 0,01$ \\
\hline & Relative weight $(x$ 100) & $0,15 \pm 0,06$ & $0,16 \pm 0,08$ & $0,09 \pm 0,04 \#$ & $0,12 \pm 0,02$ & $0,14 \pm 0,04$ & $0,20 \pm 0,06$ & $0,11 \pm 0,03$ & $0,14 \pm 0,05$ \\
\hline \multirow[t]{3}{*}{ HEART } & $N$ of mice examined & 9 & 10 & 9 & 11 & 9 & 10 & 10 & 11 \\
\hline & Absolute weight (g) & $0,28 \pm 0,09$ & $0,23 \pm 0,03$ & $0,22 \pm 0,03$ & $0,21 \pm 0,02 \#$ & $0,19 \pm 0,02$ & $0,18 \pm 0,02$ & $0,18 \pm 0,02$ & $0,18 \pm 0,02$ \\
\hline & Relative weight $(x$ 100) & $0,75 \pm 0,32$ & $0,56 \pm 0,04$ & $0,59 \pm 0,04$ & $0,53 \pm 0,04 \# \# \#$ & $0,58 \pm 0,06$ & $0,60 \pm 0,09$ & $0,63 \pm 0,08$ & $0,60 \pm 0,09$ \\
\hline \multirow[t]{3}{*}{ THYROID } & $N$ of mice examined & 10 & 9 & 7 & 11 & 9 & 9 & 8 & 10 \\
\hline & Absolute weight (g) & $0,003 \pm 0,002$ & $0,003 \pm 0,001$ & $0,003 \pm 0,002$ & $0,003 \pm 0,002$ & $0,007 \pm 0,002$ & $0,006 \pm 0,003$ & $0,004 \pm 0,001 \#$ & $0,006 \pm 0,002$ \\
\hline & Relative weight (x 100) & $0,008 \pm 0,005$ & $0,008 \pm 0,004$ & $0,007 \pm 0,004$ & $0,008 \pm 0,004$ & $0,022 \pm 0,007$ & $0,021 \pm 0,009$ & $0,016 \pm 0,005$ & $0,022 \pm 0,006$ \\
\hline \multirow[t]{3}{*}{ BRAIN } & $N$ of mice examined & 10 & 10 & 9 & 11 & 9 & 10 & 10 & 11 \\
\hline & Absolute weight (g) & $0,44 \pm 0,05$ & $0,43 \pm 0,04$ & $0,42 \pm 0,03$ & $0,42 \pm 0,04$ & $0,43 \pm 0,03$ & $0,45 \pm 0,03$ & $0,43 \pm 0,04$ & $0,43 \pm 0,05$ \\
\hline & Relative weight (x 100) & $1.16 \pm 0.18$ & $1.06 \pm 0.11$ & $1.12 \pm 0.11$ & $1.08 \pm 0.11$ & $1.37 \pm 0.16$ & $1.48 \pm 0.17$ & $1.53 \pm 0.20$ & $1.37 \pm 0.17$ \\
\hline
\end{tabular}

$\# p<0.05 ; \# \#<0.01 ; \# \# \# p<0.001$ 
Table 17 (continued): Absolute and relative organ weights of male and female mice orally treated for 42 days with different doses of BEA ( 0 - olive oil; $0.1,1$ and $10 \mathrm{mg} / \mathrm{kg}$ per day).

\begin{tabular}{|c|c|c|c|c|c|c|c|c|c|}
\hline \multirow[b]{2}{*}{ ORGAN } & & \multicolumn{4}{|c|}{ Male mice } & \multicolumn{4}{|c|}{ Female mice } \\
\hline & & Olive oil & BEA0.1 & Olive oil & BEA0.1 & Olive oil & BEA0.1 & Olive oil & BEA0.1 \\
\hline OVARIES & $\begin{array}{r}N \text { of mice examined } \\
\text { Absolute weight }(\mathrm{g}) \\
\text { Relative weight }\end{array}$ & & & & & $\begin{array}{c}9 \\
0.12 \pm 0.17 \\
0.37 \pm 0.56\end{array}$ & $\begin{array}{c}10 \\
0.07 \pm 0.02 \\
0.23 \pm 0.06\end{array}$ & $\begin{array}{c}10 \\
0.07 \pm 0.02 \\
0.24 \pm 0.09\end{array}$ & $\begin{array}{c}10 \\
0.06 \pm 0.02 \\
0.17 \pm 0.05\end{array}$ \\
\hline UTERUS & $\begin{array}{r}N \text { of mice examined } \\
\text { Absolute weight }(\mathrm{g}) \\
\text { Relative weight }(\mathrm{x} 100) \\
\end{array}$ & & & & & $\begin{array}{c}9 \\
0.25 \pm 0.09 \\
0.79 \pm 0.28\end{array}$ & $\begin{array}{c}10 \\
0.23 \pm 0.10 \\
0.76 \pm 0.31\end{array}$ & $\begin{array}{c}10 \\
0.20 \pm 0.06 \\
0.70 \pm 0.20\end{array}$ & $\begin{array}{c}11 \\
0.19 \pm 0.04 \\
0.60 \pm 0.13\end{array}$ \\
\hline TESTES & $\begin{array}{r}N \text { of mice examined } \\
\text { Absolute weight }(\mathrm{g}) \\
\text { Relative weight }(\times 100)\end{array}$ & $\begin{array}{c}10 \\
0.26 \pm 0.02 \\
0.67 \pm 0.06\end{array}$ & $\begin{array}{c}10 \\
0.26 \pm 0.05 \\
0.65 \pm 0.13\end{array}$ & $\begin{array}{c}9 \\
0.26 \pm 0.05 \\
0.70 \pm 0.15\end{array}$ & $\begin{array}{c}11 \\
0.27 \pm 0.03 \\
0.69 \pm 0.05\end{array}$ & & & & \\
\hline EPIDIDYMUS & $\begin{array}{r}N \text { of mice examined } \\
\text { Absolute weight }(\mathrm{g}) \\
\text { Relative weight }(\mathrm{x} 100)\end{array}$ & $\begin{array}{c}10 \\
0.19 \pm 0.05 \\
0.51 \pm 0.12\end{array}$ & $\begin{array}{c}10 \\
0.23 \pm 0.08 \\
0.56 \pm 0.20\end{array}$ & $\begin{array}{c}9 \\
0.24 \pm 0.02 \\
0.64 \pm 0.05\end{array}$ & $\begin{array}{c}11 \\
0.24 \pm 0.05 \\
0.61 \pm 0.10\end{array}$ & & & & \\
\hline
\end{tabular}

$\# p<0.05 ; \# \#<0.01 ; \# \#$ \# $<0.001$ 

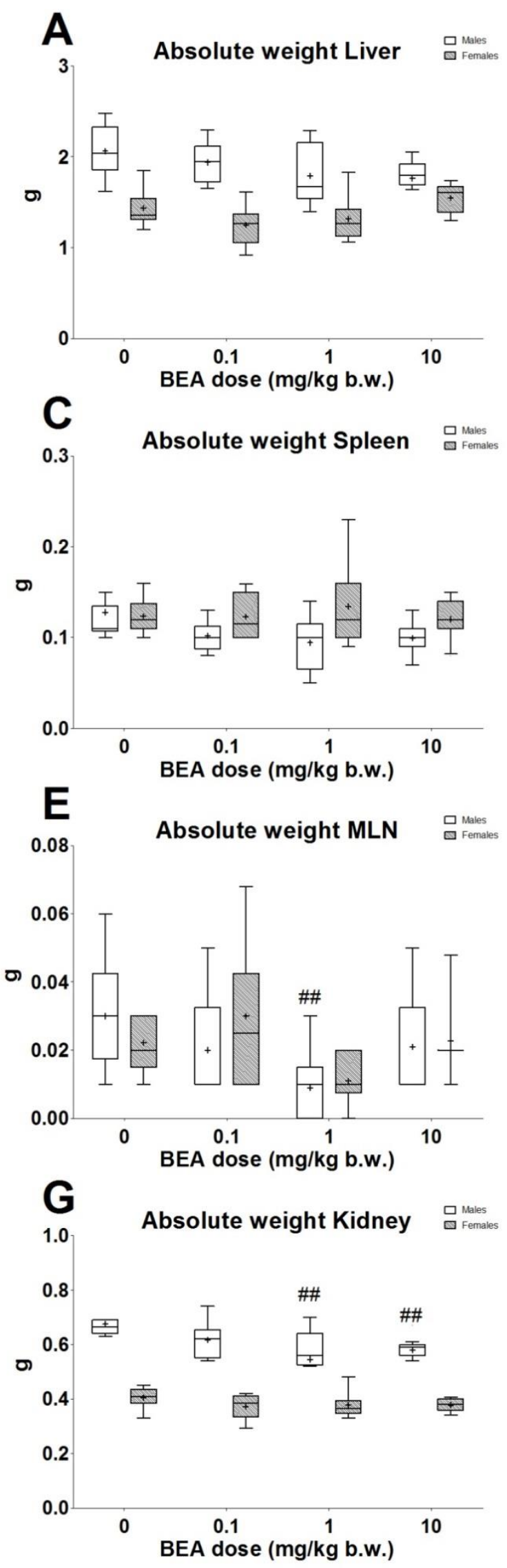
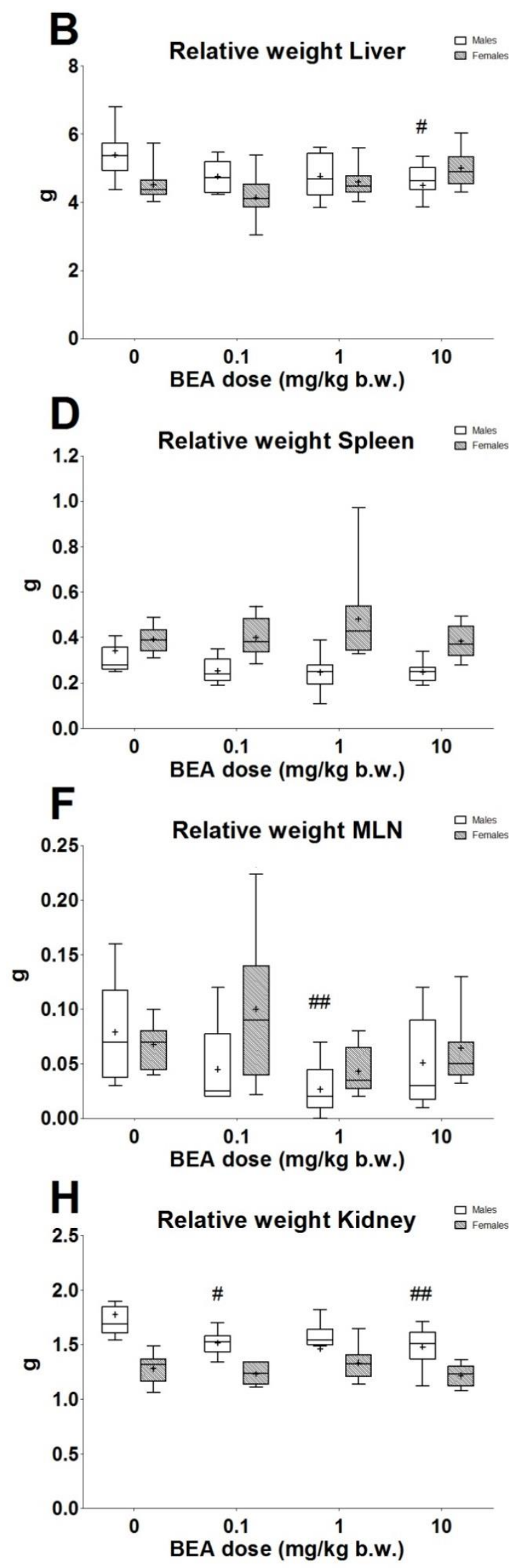

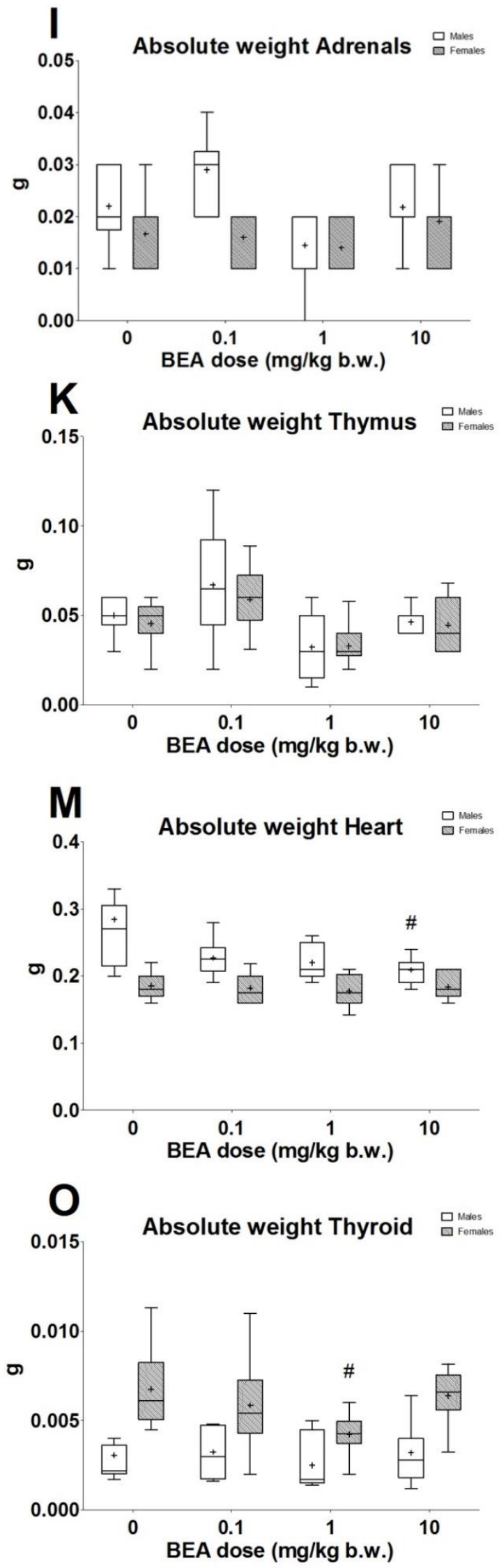
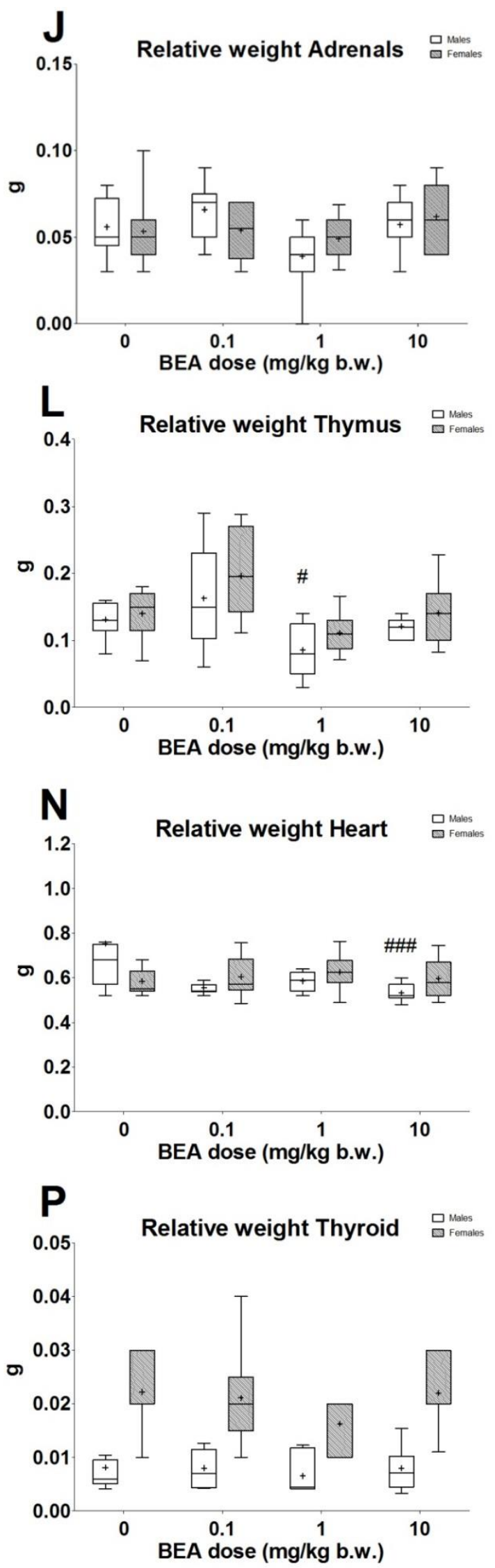

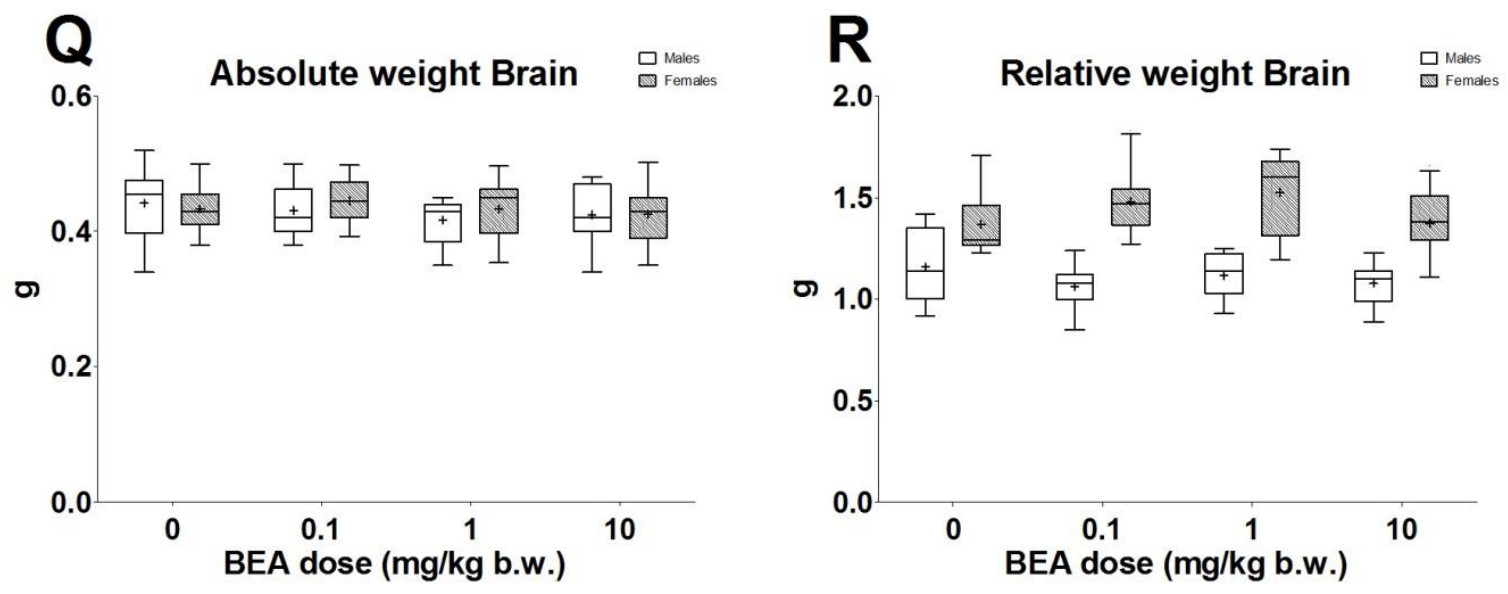

Figure 15: Absolute and relative organ weights of male (white box) and female (striped box) mice treated for 42 days orally with different doses of BEA ( 0 - olive oil; $0.1,1$ and 10 $\mathrm{mg} / \mathrm{kg}$ per day). Levels are reported as interquartile box plots, with whiskers delimiting the 10th and 90th percentiles. " + " indicates the mean value for each experimental group; \# $\mathrm{p}$ $<0.05$; \#\# $\mathrm{p}<0.01$; \#\# $\mathrm{p}<0.001$.

Absolute kidney weight was significantly decreased in males at BEA1 and BEA10 (Fig. 15G) whereas relative weight was decreased in male mice at BEA0.1 and BEA10 (Fig. 15H). In females, kidney weight was unaffected by BEA treatment.

Adrenals weight was unaffected by BEA treatment in all groups (Fig. 15I-J).

Significant decrease in thymus relative weight of BEA1-treated male mice was observed (Fig. 15L). No effect was recorded in female mice.

Heart weight was decreased both as absolute and relative value in BEA10 treated male mice (Fig. $15 \mathrm{M}-\mathrm{N})$. No effect was observed in female mice.

Absolute thyroid weight was decreased by BEA1 in female mice (Fig. 150). No effect was recorded in males.

Brain weight was unaffected in both female and male mice (Fig. 15Q-R).

As regards reproductive organs, testis and epididymis weight were unaffected in male mice by BEA treatment at all doses (Fig. 16A-D). Similarly, in females, uterus and ovary weight was unaffected by BEA treatment (Fig. 16E-H). 

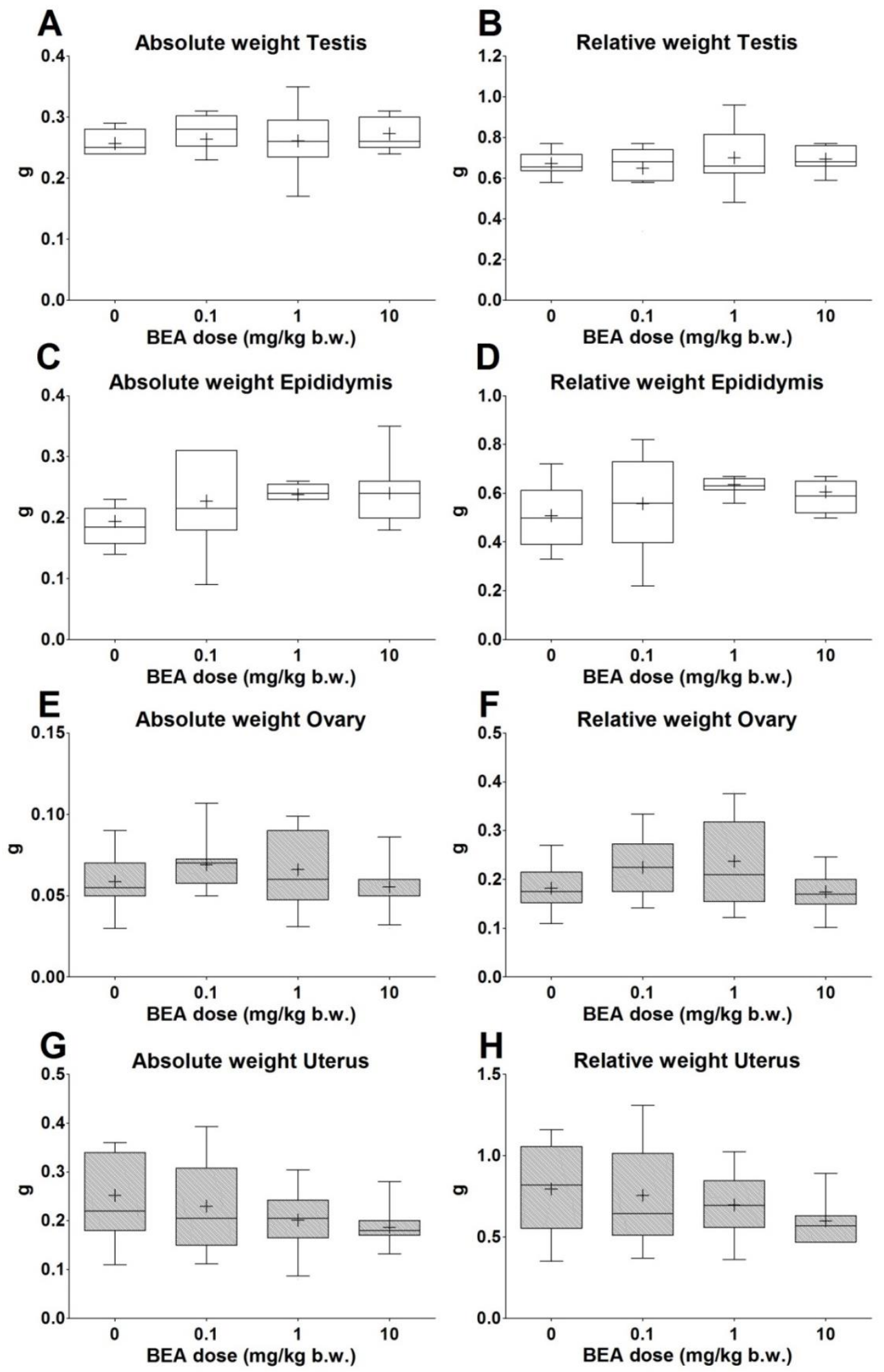

Figure 16: Absolute and relative weight of reproductive organs of male (white box) and female (striped box) mice orally treated for 42 days with different doses of BEA ( 0 - olive oil; 0.1 , 1 and $10 \mathrm{mg} / \mathrm{kg}$ per day). Levels are reported as interquartile box plots, with whiskers delimiting the 10th and 90th percentiles. "+" indicates the mean value for each experimental group. 


\subsubsection{BEA - Histopathological endpoints}

The histopathological data are summarized in Table 18. 
Table 18: Incidence of each histopathological endpoint analyzed in male and female mice orally treated for 42 days with different doses of BEA (0 olive oil; $0.1,1$ and $10 \mathrm{mg} / \mathrm{kg}$ per day).

\begin{tabular}{|c|c|c|c|c|c|c|c|c|}
\hline \multirow{2}{*}{ ORGAN/Effect } & \multicolumn{4}{|c|}{ Males } & \multicolumn{4}{|c|}{ Females } \\
\hline & Olive oil & BEA0.1 & BEA1 & BEA10 & Olive oil & BEA0.1 & BEA1 & BEA10 \\
\hline THYROID & 7 & 7 & 5 & 7 & 6 & 7 & 7 & 6 \\
\hline 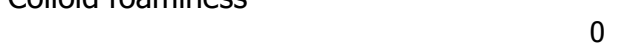 & 3 & 2 & 3 & 6 & 2 & 3 & 6 & 4 \\
\hline 1 & 3 & 5 & 2 & & 4 & 4 & 1 & 2 \\
\hline 2 & & & & 1 & & & & \\
\hline 3 & 1 & & & & & & & \\
\hline Total Finding Incidence & 4 & 5 & 2 & 1 & 4 & 4 & 1 & 2 \\
\hline Necrotic cells exfoliated into the colloid & & & & & & & & \\
\hline 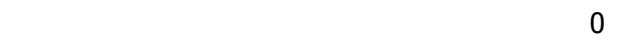 & 6 & 5 & 4 & 7 & 6 & 7 & 6 & 5 \\
\hline 1 & 1 & 2 & 1 & & & & 1 & 1 \\
\hline \begin{tabular}{ll} 
Total Finding Incidence \\
\cline { 2 - 3 }
\end{tabular} & 1 & 2 & 1 & 0 & 0 & 0 & 1 & 1 \\
\hline Colloid reduction/absence in follicles & & & & & & & & \\
\hline ( & 7 & 7 & 1 & & 5 & 4 & 3 & 1 \\
\hline 1 & & & 2 & & 1 & 2 & 2 & 4 \\
\hline 2 & & & 1 & 2 & & 1 & 2 & \\
\hline 3 & & & 1 & 3 & & & & 1 \\
\hline 4 & & & & 2 & & & & \\
\hline Total Finding Incidence & 0 & 0 & 4\# & 7\#\# & 1 & 3 & 4 & 5 \\
\hline Pyknotic nuclei & & & & & & & & \\
\hline r & 7 & 7 & 2 & & 6 & 7 & 4 & 1 \\
\hline 1 & & & 2 & 3 & & & 2 & 3 \\
\hline 2 & & & 1 & 3 & & & 1 & 2 \\
\hline 4 & & & & 1 & & & & \\
\hline \begin{tabular}{ll} 
Total Finding Incidence \\
\cline { 2 - 2 }
\end{tabular} & 0 & 0 & 3 & 7\#\# & 0 & 0 & 3 & $5 \#$ \\
\hline Follicles degeneration & & & & & & & & \\
\hline-5 & 7 & 7 & 5 & 3 & 6 & 7 & 5 & 4 \\
\hline 1 & & & & 1 & & & 1 & 2 \\
\hline 2 & & & & 1 & & & 1 & \\
\hline 4 & & & & 2 & & & & \\
\hline Total Finding Incidence & 0 & 0 & 0 & 4 & 0 & 0 & 2 & 2 \\
\hline
\end{tabular}

Grade 0: No change; Grade 1: Minimal; Grade 2: Mild; Grade 3: Moderate; Grade 4: Marked. \#p < 0.05; \#\# p $<0.01$. 
Table 18 (continued): Incidence of each histopathological endpoint analyzed in male and female mice orally treated for 42 days with different doses of BEA ( 0 - olive oil; $0.1,1$ and $10 \mathrm{mg} / \mathrm{kg}$ per day).

\begin{tabular}{|c|c|c|c|c|c|c|c|c|}
\hline & \multicolumn{4}{|c|}{ Males } & \multicolumn{4}{|c|}{ Females } \\
\hline ORGAN/Effect & Olive oil & BEA0.1 & BEA1 & BEA10 & Olive oil & BEA0.1 & BEA1 & BEA10 \\
\hline $\begin{array}{ll}\text { DUODENUM } & N \text { of mice examined } \\
\text { Enterocytes vacuolation } & \end{array}$ & 7 & 7 & 6 & 7 & 7 & 7 & 6 & 7 \\
\hline $\begin{array}{r}0 \\
1 \\
2 \\
3 \\
\text { Total Finding Incidence }\end{array}$ & $\begin{array}{l}3 \\
3 \\
1 \\
4\end{array}$ & $\begin{array}{l}3 \\
3 \\
1 \\
4\end{array}$ & $\begin{array}{l}3 \\
1 \\
2 \\
3\end{array}$ & $\begin{array}{l}5 \\
1 \\
1 \\
2\end{array}$ & 7 & $\begin{array}{l}5 \\
1 \\
1 \\
2\end{array}$ & $\begin{array}{c}2 \\
1 \\
3 \\
4 \#\end{array}$ & $\begin{array}{l}2 \\
2 \\
1 \\
2 \\
5\end{array}$ \\
\hline \begin{tabular}{lr} 
HEART & $N$ of mice examined \\
Inflammatory cell foci & \\
& \\
& \\
& Total Finding Incidence \\
\cline { 2 - 2 }
\end{tabular} & $\begin{array}{l}7 \\
6 \\
1 \\
1\end{array}$ & $\begin{array}{l}7 \\
7 \\
0\end{array}$ & $\begin{array}{l}6 \\
6 \\
0\end{array}$ & $\begin{array}{l}7 \\
7 \\
0\end{array}$ & $\begin{array}{l}7 \\
7 \\
0\end{array}$ & $\begin{array}{l}7 \\
7 \\
0\end{array}$ & $\begin{array}{l}6 \\
6 \\
0\end{array}$ & $\begin{array}{l}7 \\
7 \\
0\end{array}$ \\
\hline $\begin{array}{r}\text { Chronic inflammation } \\
\\
1 \\
2 \\
3 \\
\\
\text { Total Finding Incidence }\end{array}$ & 7 & $\begin{array}{l}6 \\
1 \\
1\end{array}$ & 6 & 7 & $\begin{array}{l}6 \\
1\end{array}$ & 7 & $\begin{array}{l}5 \\
1 \\
1\end{array}$ & 7 \\
\hline
\end{tabular}

Grade 0: No change; Grade 1: Minimal; Grade 2: Mild; Grade 3: Moderate; Grade 4: Marked. \#p < 0.05 
Table 18 (continued): Incidence of each histopathological endpoint analyzed in male and female mice orally treated for 42 days with different doses of BEA (0 - olive oil; $0.1,1$ and $10 \mathrm{mg} / \mathrm{kg}$ per day).

\begin{tabular}{|c|c|c|c|c|c|c|c|c|}
\hline & \multicolumn{4}{|c|}{ Males } & \multicolumn{4}{|c|}{ Females } \\
\hline ORGAN/Effect & Olive oil & BEA0.1 & BEA1 & BEA10 & Olive oil & BEA0.1 & BEA1 & BEA10 \\
\hline$N$ of mice examined & 7 & 7 & 6 & 7 & 7 & 7 & 7 & 7 \\
\hline $\begin{array}{r}0 \\
1 \\
2 \\
\text { Total Finding Incidence } \\
\end{array}$ & $\begin{array}{l}2 \\
3 \\
2 \\
5\end{array}$ & $\begin{array}{l}4 \\
2 \\
1 \\
3\end{array}$ & $\begin{array}{l}1 \\
4 \\
1 \\
5\end{array}$ & $\begin{array}{l}5 \\
1 \\
1 \\
2\end{array}$ & $\begin{array}{l}3 \\
3 \\
1 \\
4\end{array}$ & $\begin{array}{l}6 \\
1 \\
1\end{array}$ & $\begin{array}{l}2 \\
2 \\
3 \\
5\end{array}$ & $\begin{array}{l}1 \\
2 \\
4 \\
6\end{array}$ \\
\hline $\begin{array}{r}\text { Hepatocytic vacuolation } \\
0 \\
1 \\
2 \\
3 \\
\text { Total Finding Incidence }\end{array}$ & 7 & 7 & $\begin{array}{l}5 \\
1\end{array}$ & $\begin{array}{l}6 \\
1\end{array}$ & $\begin{array}{l}5 \\
1 \\
1 \\
2\end{array}$ & $\begin{array}{l}5 \\
2 \\
2\end{array}$ & 7 & $\begin{array}{l}5 \\
1 \\
1 \\
2\end{array}$ \\
\hline $\begin{array}{rr}\text { Inflammatory cell foci } & 0 \\
& 1 \\
& \text { Total Finding Incidence } \\
\end{array}$ & $\begin{array}{l}7 \\
0 \\
\end{array}$ & $\begin{array}{l}7 \\
0 \\
\end{array}$ & $\begin{array}{l}6 \\
0 \\
\end{array}$ & $\begin{array}{l}5 \\
2 \\
2 \\
\end{array}$ & $\begin{array}{l}5 \\
2 \\
2 \\
\end{array}$ & $\begin{array}{l}6 \\
1 \\
1 \\
\end{array}$ & $\begin{array}{l}6 \\
1 \\
1 \\
\end{array}$ & $\begin{array}{l}5 \\
2 \\
2 \\
\end{array}$ \\
\hline $\begin{array}{lr}\text { SPLEEN } & N \text { of mice examined } \\
\text { Extramedullary haematopoiesis } & 0 \\
& 1 \\
& \\
& \text { Total Finding Incidence }\end{array}$ & $\begin{array}{l}4 \\
1 \\
5\end{array}$ & $\begin{array}{l}1 \\
4 \\
5\end{array}$ & $\begin{array}{l}2 \\
2 \\
4\end{array}$ & $\begin{array}{l}3 \\
3 \\
6\end{array}$ & $\begin{array}{l}4 \\
4\end{array}$ & $\begin{array}{l}1 \\
4 \\
5\end{array}$ & $\begin{array}{l}5 \\
1 \\
2 \\
2 \\
4\end{array}$ & $\begin{array}{l}2 \\
3 \\
5\end{array}$ \\
\hline
\end{tabular}

Grade 0: No change; Grade 1: Minimal; Grade 2: Mild; Grade 3: Moderate; Grade 4: Marked. 
Table 18 (continued): Incidence of each histopathological endpoint analyzed in male and female mice orally treated for 42 days with different doses of BEA (0 - olive oil; $0.1,1$ and $10 \mathrm{mg} / \mathrm{kg}$ per day).

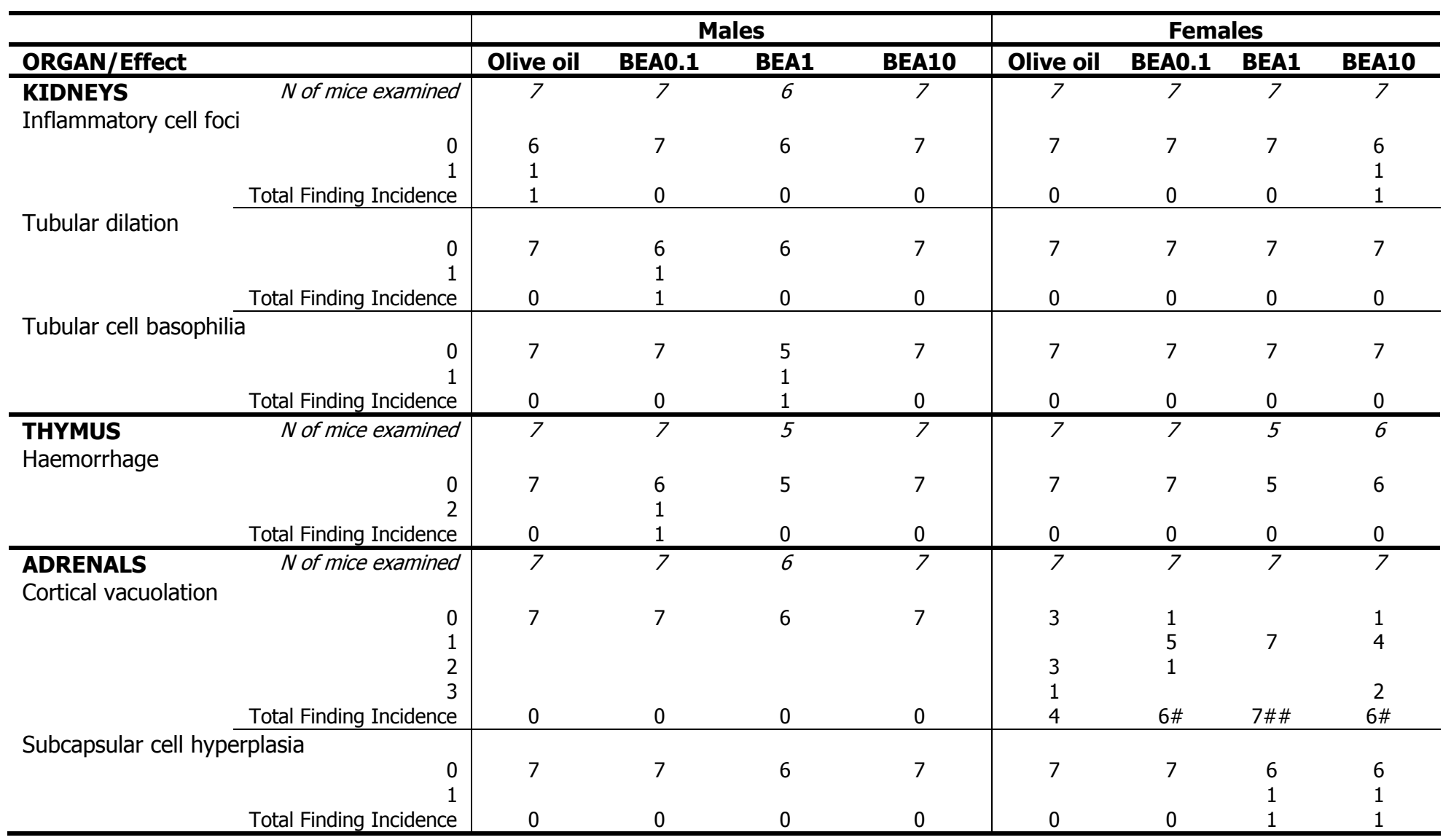

Grade 0: No change; Grade 1: Minimal; Grade 2: Mild; Grade 3: Moderate; Grade 4: Marked. \#p < 0.05; \#\# p $<0.01$. 
Table 18(continued): Incidence of each histopathological endpoint analyzed in male and female mice orally treated for 42 days with different doses of BEA (0 - olive oil; $0.1,1$ and $10 \mathrm{mg} / \mathrm{kg}$ per day).

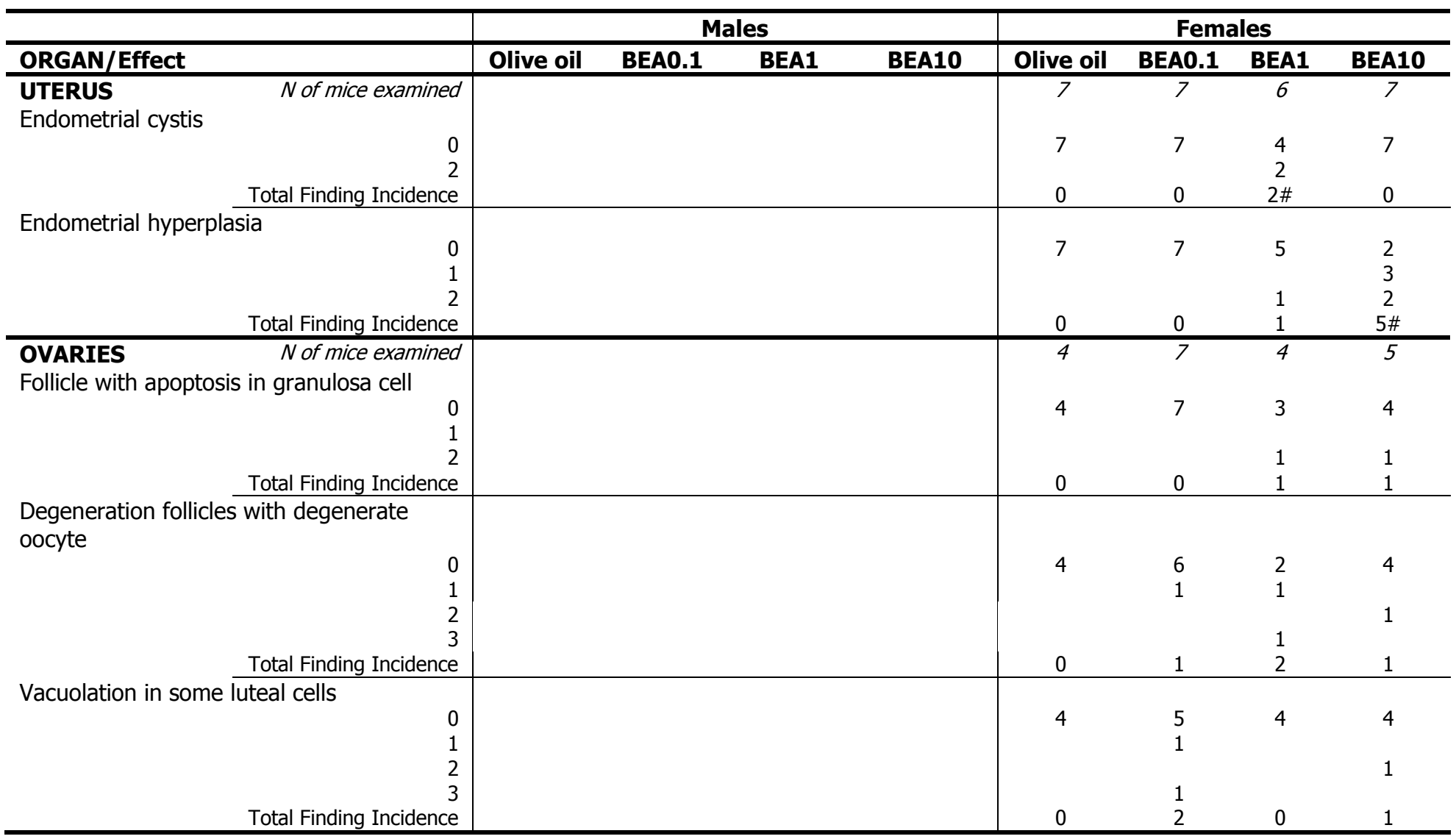

Grade 0: No change; Grade 1: Minimal; Grade 2: Mild; Grade 3: Moderate; Grade 4: Marked. \#p < 0.05. 
Table 18(continued): Incidence of each histopathological endpoint analyzed in male and female mice orally treated for 42 days with different doses of BEA (0 - olive oil; $0.1,1$ and $10 \mathrm{mg} / \mathrm{kg}$ per day).

\begin{tabular}{|c|c|c|c|c|c|c|c|c|c|}
\hline \multirow{2}{*}{ ORGAN/Effect } & & \multicolumn{4}{|c|}{ Males } & \multicolumn{4}{|c|}{ Females } \\
\hline & & Olive oil & BEA0.1 & BEA1 & BEA10 & Olive oil & BEA0.1 & BEA1 & BEA10 \\
\hline \multirow{3}{*}{$\begin{array}{l}\text { EPIDIDYMIS } \\
\text { Cellular debris }\end{array}$} & $N$ of mice examined & 7 & 8 & 7 & 7 & & & & \\
\hline & 1 & 1 & 4 & 1 & 1 & & & & \\
\hline & Total Finding Incidence & 1 & 4 & 1 & 1 & & & & \\
\hline \multicolumn{6}{|c|}{ Caput: epithelial vacuolation } & & & & \\
\hline & 2 & & & 2 & & & & & \\
\hline & Total Finding Incidence & 1 & 0 & 2 & 3 & & & & \\
\hline \multicolumn{6}{|c|}{ Tubules degeneration } & & & & \\
\hline & 0 & 7 & 8 & 5 & 4 & & & & \\
\hline & 1 & & & 1 & 3 & & & & \\
\hline & 2 & & & 1 & & & & & \\
\hline & Total Finding Incidence & 0 & 0 & 2 & 3 & & & & \\
\hline & $\%$ Atrophic tubules & $0.0 \pm 0.0$ & $0.0 \pm 0.0$ & $0.0 \pm 0.0$ & $22.9 \pm 9.7 \#$ & & & & \\
\hline & $\%$ Tubules with vacuoles & $0.0 \pm 0.0$ & $7.1 \pm 3.6$ & $1.7 \pm 1.7$ & $7.9 \pm 4.1$ & & & & \\
\hline
\end{tabular}

*Testis: 50 different tubules/mice were observed and the scoring of lesions was done quantitatively, using percentages. 
Table 18 (continued): Incidence of each histopathological endpoint analyzed in male and female mice orally treated for 42 days with different doses of BEA (0 - olive oil; $0.1,1$ and $10 \mathrm{mg} / \mathrm{kg}$ per day).

\begin{tabular}{|c|c|c|c|c|c|c|c|c|c|}
\hline & & \multicolumn{4}{|c|}{ Males } & \multicolumn{4}{|c|}{ Females } \\
\hline \multicolumn{2}{|l|}{ ORGAN/Effect } & Olive oil & BEA0.1 & BEA1 & BEA10 & Olive oil & BEA0.1 & BEA1 & BEA10 \\
\hline \multirow{3}{*}{$\begin{array}{l}\text { BRAIN } \\
\text { Lymphatic } \\
\text { infiltration }\end{array}$} & $N$ of mice examined & 10 & 10 & 7 & 11 & 9 & 9 & 10 & 11 \\
\hline & $\begin{array}{r}0 \\
1 \\
2 \\
4\end{array}$ & 10 & $\begin{array}{l}9 \\
1\end{array}$ & 7 & 11 & $\begin{array}{l}8 \\
1\end{array}$ & $\begin{array}{l}8 \\
1\end{array}$ & $\begin{array}{l}6 \\
3 \\
1\end{array}$ & $\begin{array}{l}6 \\
2 \\
3\end{array}$ \\
\hline & Total Finding Incidence & 0 & 1 & 0 & 0 & 1 & 1 & 4 & 5 \\
\hline \multirow[t]{4}{*}{ Spongiosis } & 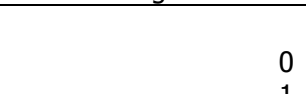 & 5 & 5 & 5 & 4 & 2 & 6 & 3 & 4 \\
\hline & $\begin{array}{l}1 \\
2\end{array}$ & 4 & 5 & 2 & $\begin{array}{l}1 \\
6\end{array}$ & 4 & 3 & 4 & 5 \\
\hline & $\begin{array}{l}3 \\
4\end{array}$ & 1 & & & & 3 & & 3 & $\begin{array}{l}1 \\
1\end{array}$ \\
\hline & Total Finding Incidence & 5 & 5 & 2 & 7 & 7 & 3 & 7 & 7 \\
\hline
\end{tabular}

Grade 0: No change; Grade 1: Minimal; Grade 2: Mild; Grade 3: Moderate; Grade 4: Marked. 
No histopathological alterations were present in MLN (for both sexes) and mammary gland in all the mice analyzed (data not shown).

Histopathological analysis of thyroid showed reduced colloid in males at BEA1 and BEA10 (Table 18). Pyknotic nuclei were increased in both male and female mice at BEA10 (Table 18). Histomorphometrical analysis showed no alteration of the thyroid follicles number and density as well as of the thyroid area (Fig. 17A-C).
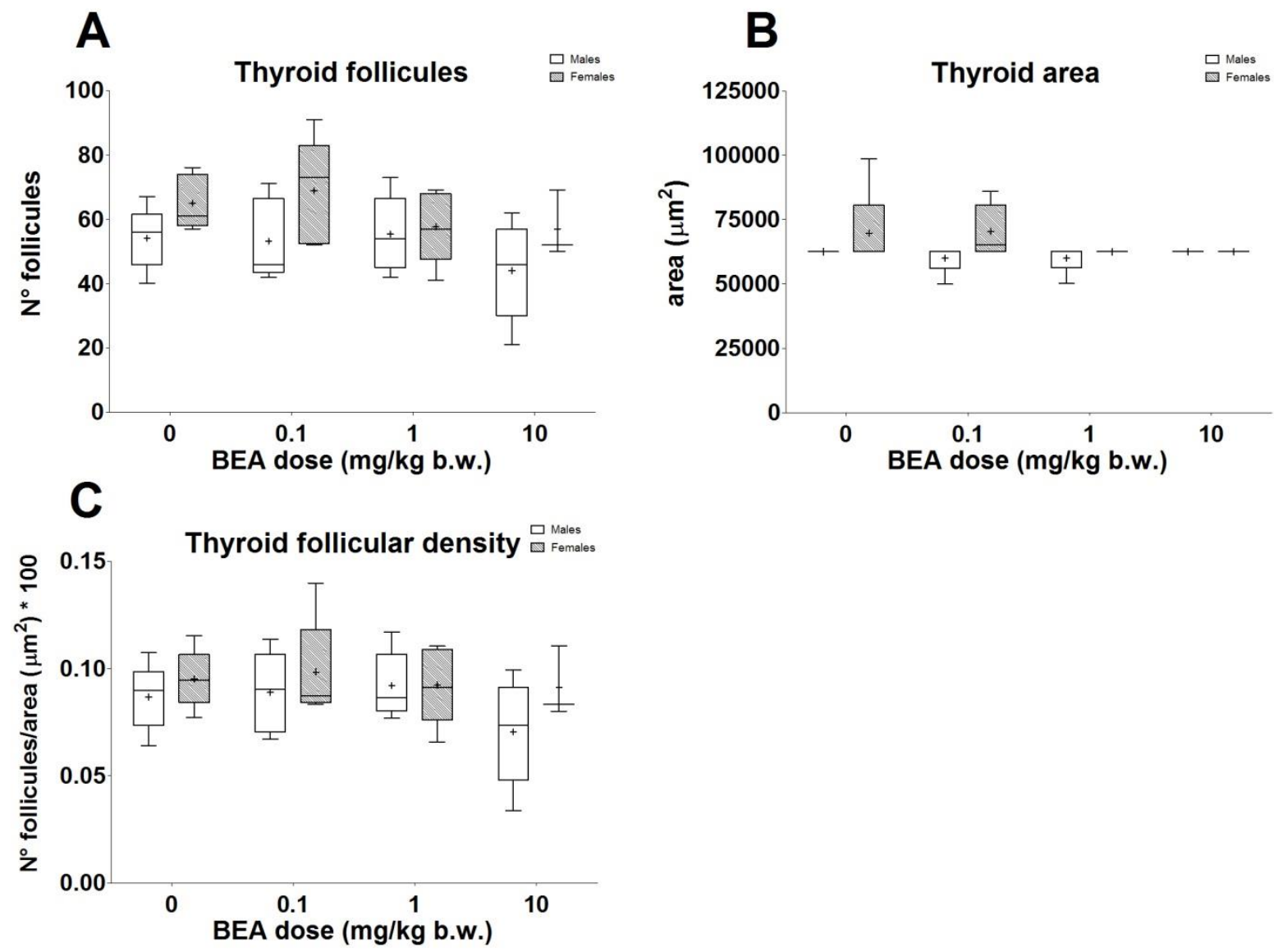

Figure 17: Histomorphometrical measurement of number of follicles (A) and thyroid area (B); calculation follicular density (C) of thyroid in male (white box) and female (striped box) mice orally treated for 42 days with different doses of BEA ( 0 - olive oil; $0.1,1$ and 10 $\mathrm{mg} / \mathrm{kg}$ per day). Levels are reported as interquartile box plots, with whiskers delimiting the 10 th and 90th percentiles. " + " indicates the mean value for each experimental group.

Histopathological analysis of the duodenum showed enterocytes vacuolization observed in BEA1 females (Table 18), the correlation with the treatment is unclear. No significant effects were observed in male mice.

No histopathological effects were observed in heart and liver of male and female mice (Table 18).

In spleen, no histopathological effects were observed in male and female mice (Table 18). Red pulp area was increased in male mice at BEA0.1 (Fig. 18A) and the white pulp area increased at BEA0.1 and BEA10 dose levels without a clear dose-response curve (Fig. 18B); the correlation with the treatment is unclear. No effect on the red/white ratio was recorded (Fig 18C). 
A

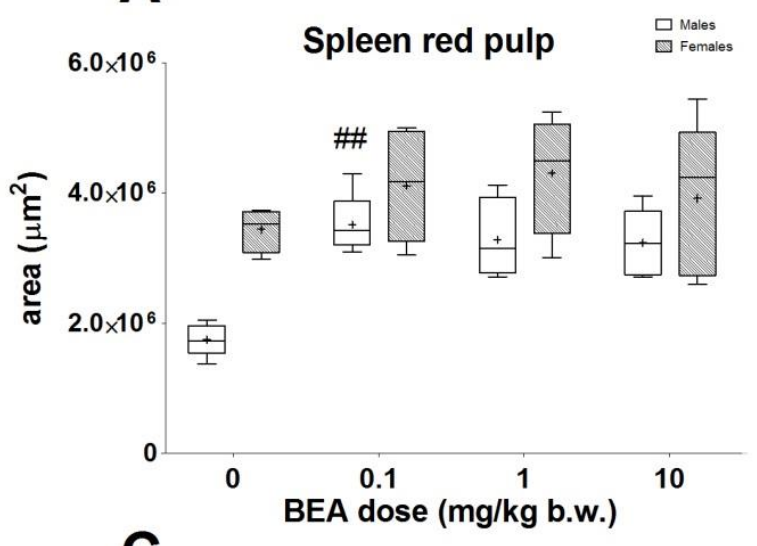

C

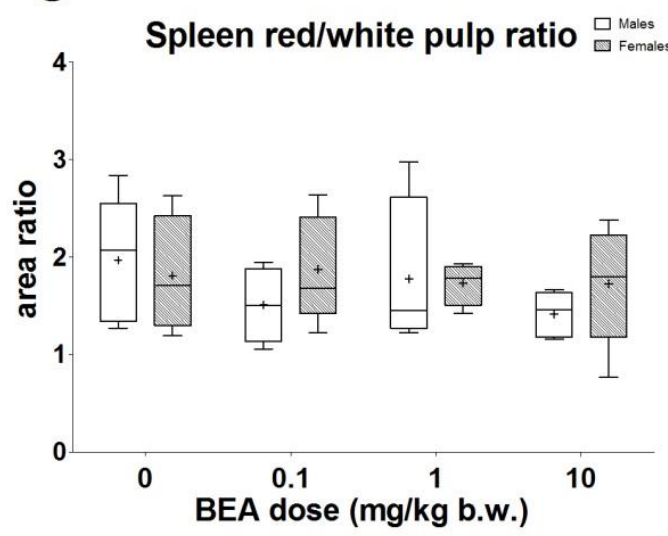

B

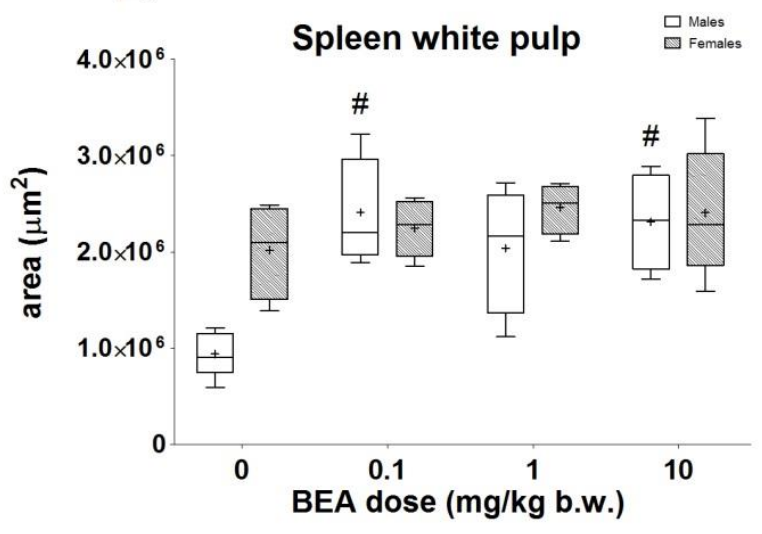

Figure 18: Histomorphometrical measurement of spleen red pulp area $(A)$ and white pulp area (B); calculation ratio of the red to white areas (C) of spleen in male (white box) and female (striped box) mice orally treated for 42 days with different doses of BEA ( 0 - olive oil; $0.1,1$ and $10 \mathrm{mg} / \mathrm{kg}$ per day). Levels are reported as interquartile box plots, with whiskers delimiting the 10th and 90th percentiles. " + " indicates the mean value for each experimental group. \# $\mathrm{p}<0.05 ; \# \# \mathrm{p}<0.01$.

In kidney, no histopathological effects were observed in male and female mice (Table 18).No histopathological effect was recorded on thymus in male and female mice (Table 18). Moreover, histomorphometrical analysis of cortex and medulla area did not show any alteration (Fig. 19A-C). 

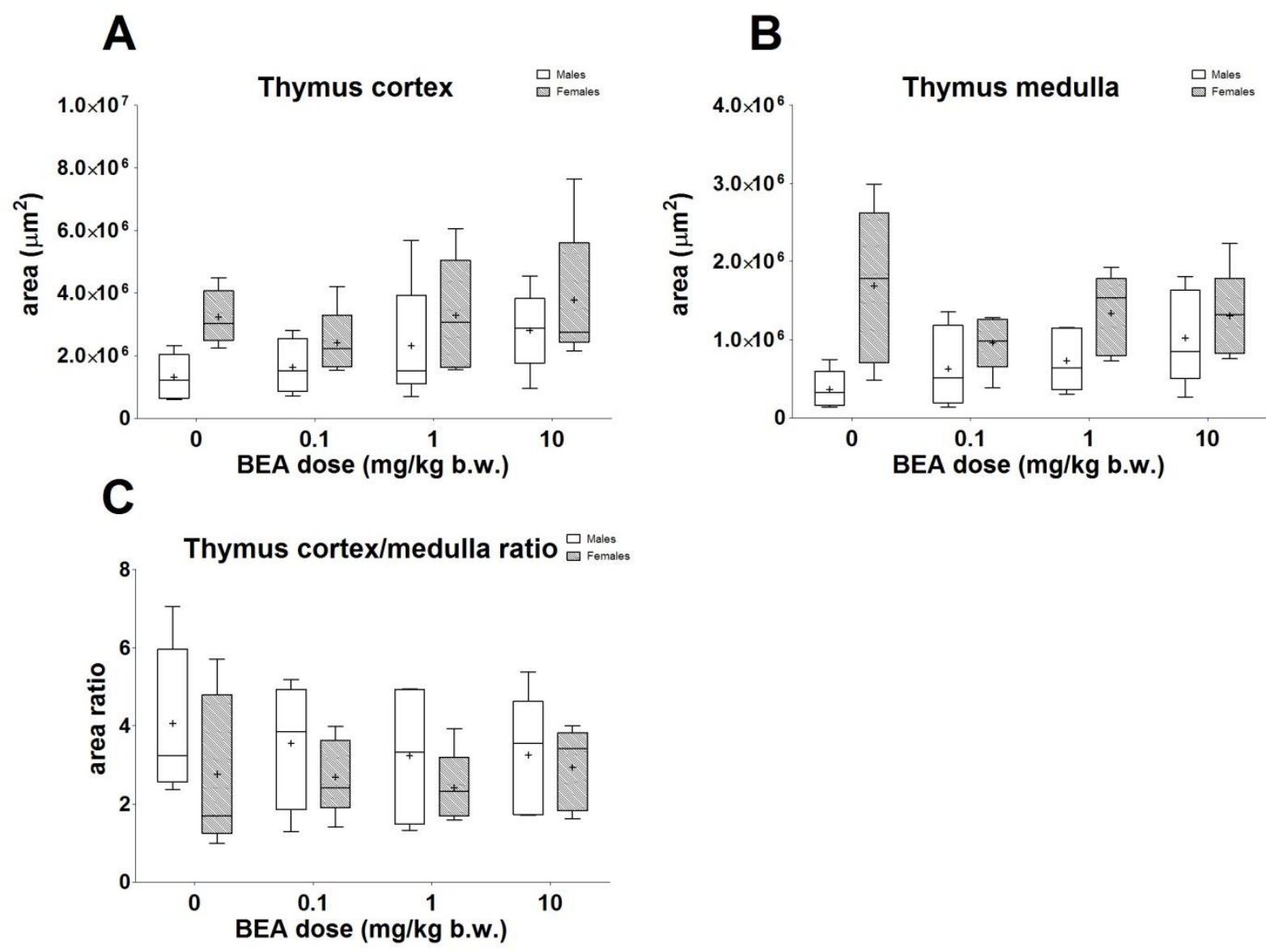

Figure 19: Histomorphometrical measurement of cortex (A) and medulla (B) thymus areas; calculation ratio of the cortex to medulla areas (C) of thymus in male (white box) and female (striped box) mice orally treated for 42 days with different doses of BEA ( 0 - olive oil; $0.1,1$ and $10 \mathrm{mg} / \mathrm{kg}$ per day). Levels are reported as interquartile box plots, with whiskers delimiting the 10 th and 90th percentiles. " + " indicates the mean value for each experimental group.

Adrenal cortical vacuolization was present in female mice at all dose levels (Table 18) whereas no effect was recorded in male mice. No effect was observed in the cortex and medulla areas in both sexes (Fig. 20A-C). 
A
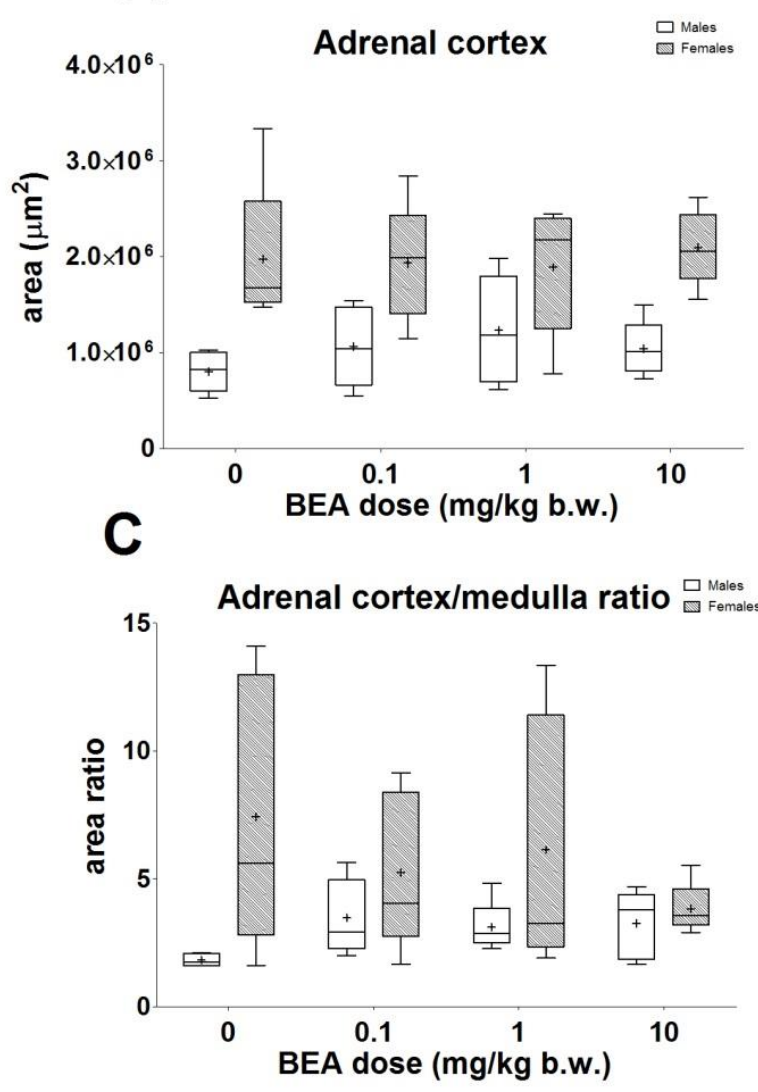

B

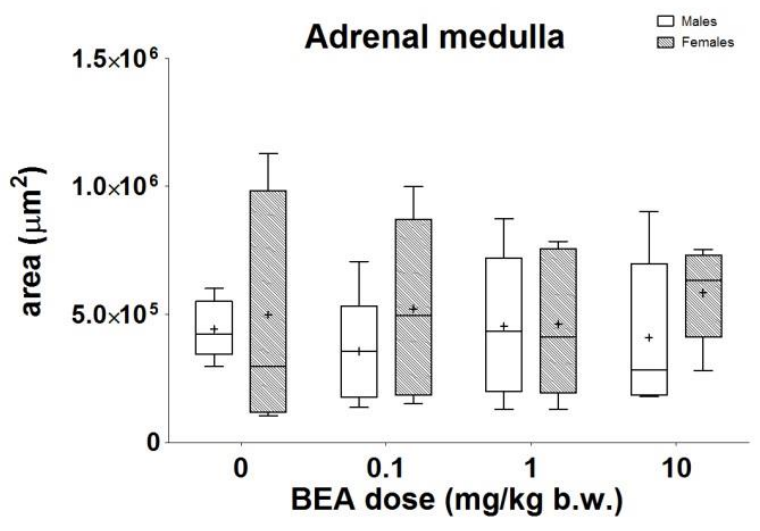

Figure 20: Histomorphometrical measurement of cortex (A) and medulla (B) adrenal areas; calculation ratio of the cortex to medulla adrenal areas (C) of adrenals in male (white box) and female (striped box) mice orally treated for 42 days with different doses of BEA ( 0 olive oil; $0.1,1$ and $10 \mathrm{mg} / \mathrm{kg}$ per day). Levels are reported as interquartile box plots, with whiskers delimiting the 10 th and 90 th percentiles. " + " indicates the mean value for each experimental group.

In brain, no histopathological effect was observed in both male and female mice (Table 18).

In females, the presence of endometrial cysts in uteri was significantly higher at BEA1, the correlation with the treatment is unclear; endometrial hyperplasia was increased at BEA10 (Table 18). Moreover, the myometrial area decreased at BEA0.1 (Fig. 21B). The areas of other uterus components were unaffected (Fig. 21A-C). 
A

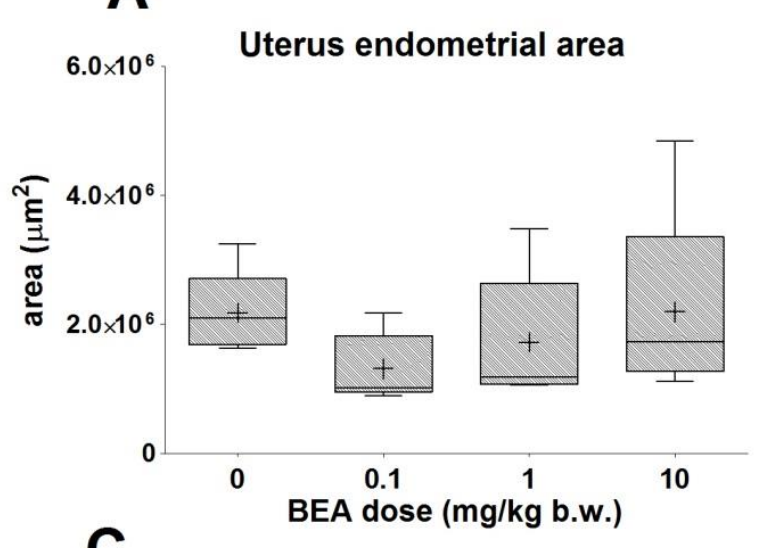

C

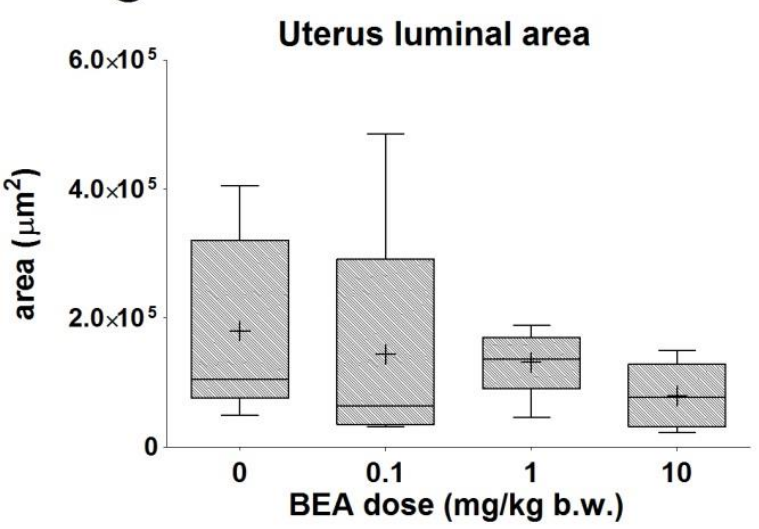

B

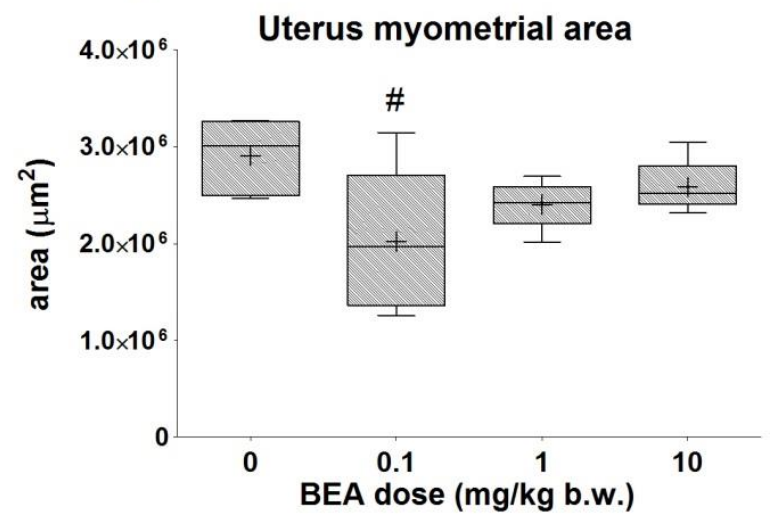

Figure 21: Histomorphometrical measurement of endometrial $(A)$ myometrial $(B)$ and luminal $(C)$ uterus areas in female mice orally treated for 42 days with different doses of BEA ( 0 olive oil; $0.1,1$ and $10 \mathrm{mg} / \mathrm{kg}$ per day). Levels are reported as interquartile box plots, with whiskers delimiting the 10th and 90th percentiles. " + " indicates the mean value for each experimental group. \# $\mathrm{p}<0.05$.

Histopathological and histomorphometrical analyses of ovary did not show any alterations (Table 18 and Fig. 22A-C). 


\section{A}
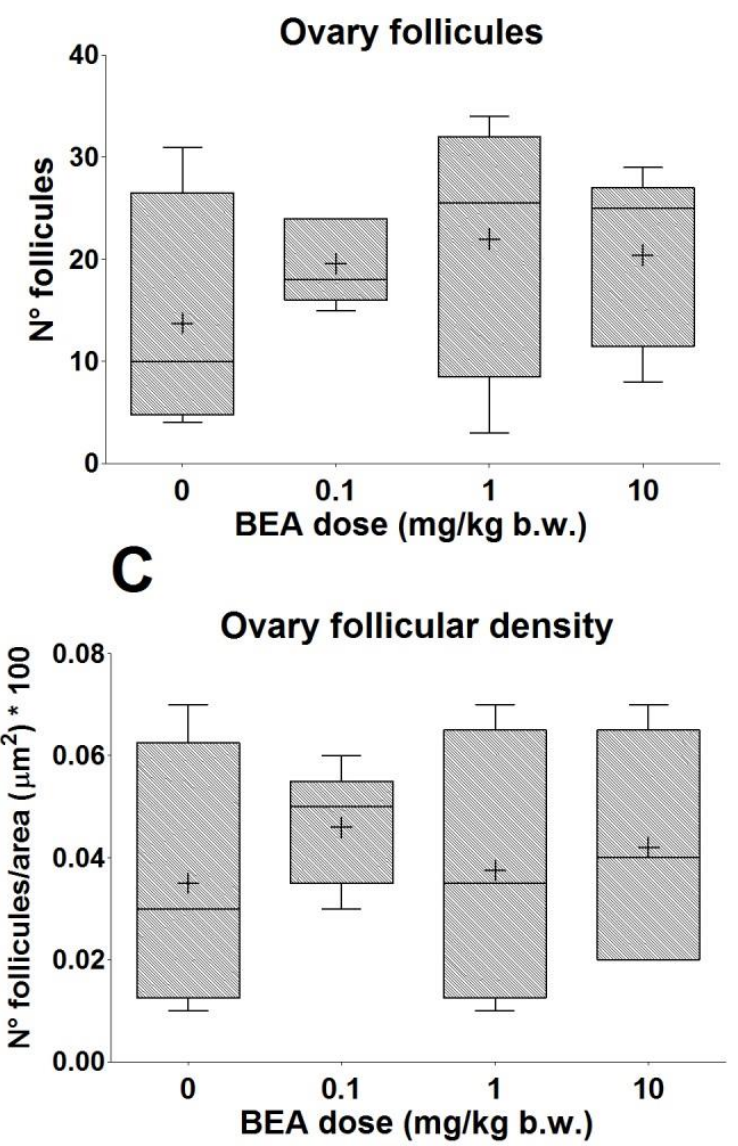

B

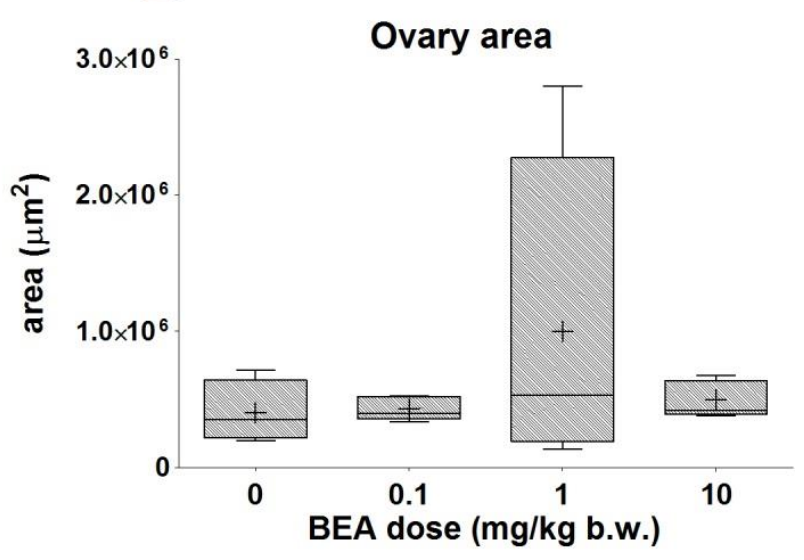

Figure 22: Histomorphometrical measurement of follicular number (A) and ovary area (B); calculation follicular density $(C)$ of ovaries in female mice orally treated for 42 days with different doses of BEA ( 0 - olive oil; $0.1,1$ and $10 \mathrm{mg} / \mathrm{kg}$ per day). Levels are reported as interquartile box plots, with whiskers delimiting the 10th and 90th percentiles. "+" indicates the mean value for each experimental group.

In testes, the percentage of unaffected tubules was significantly decreased at BEA10 whereas the percentage of atrophic and with germ cell disorganization tubules were significantly increased at BEA10 (Table 18). Histomorphometrical measurement of testis areas did not show any significant alteration (Fig. 23). 

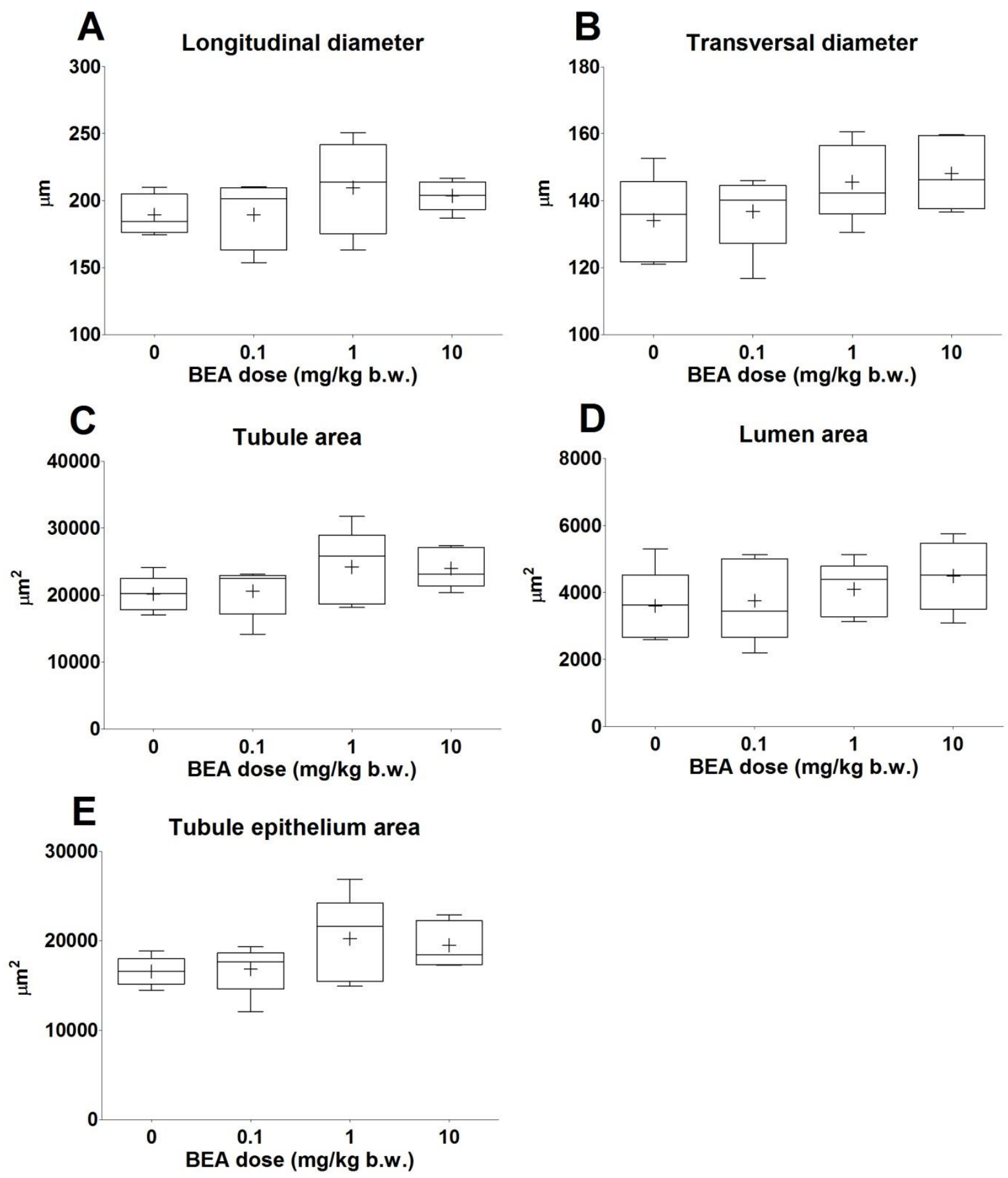

Figure 23: Histomorphometrical measurement of longitudinal (A) and transversal (B) testis diameter, testis tubule (C) lumen (D) and epithelium (E) areas in male mice orally treated for 42 days with different doses of BEA ( 0 - olive oil; $0.1,1$ and $10 \mathrm{mg} / \mathrm{kg}$ per day). Levels are reported as interquartile box plots, with whiskers delimiting the 10th and 90th percentiles. " + " indicates the mean value for each experimental group.

\subsubsection{BEA - Biomarker endpoints}

It should be noted that the effects recorded on biomarkers were statistically different from the paired CTRL group without a clear dose-response relationship. 
In general, considering the available numerosity and the mice inter-individual variability, it is not surprising to observe wide standard deviations for some of the endpoints analyzed. Since data distribution was not Gaussian, a non parametric analysis based on ranks has been performed which is most conservative and avoid to obtain false positives, especially for reduced numerosity of groups. The analysis has been repeated excluding the single data more divergent and the significance remained (data not shown), confirming that tests based on ranks assure a sound statistics when numerosity is not elevated. Indeed, since the biological relevance of isolated effects is not clear, they are not considered in the NOAEL definition.

\section{Liver and kidney serum biomarkers}

AST and ALT liver biomarkers were unaffected by BEA treatment in both sexes (Fig. 24A-B). Different effects were observed for kidney biomarkers: creatinine (CRE) was significantly increased in BEA1treated males, the correlation with the treatment is unclear. Blood urea nitrogen (BUN) was significantly increased in BEA10-treated females (Fig. 24C-D).

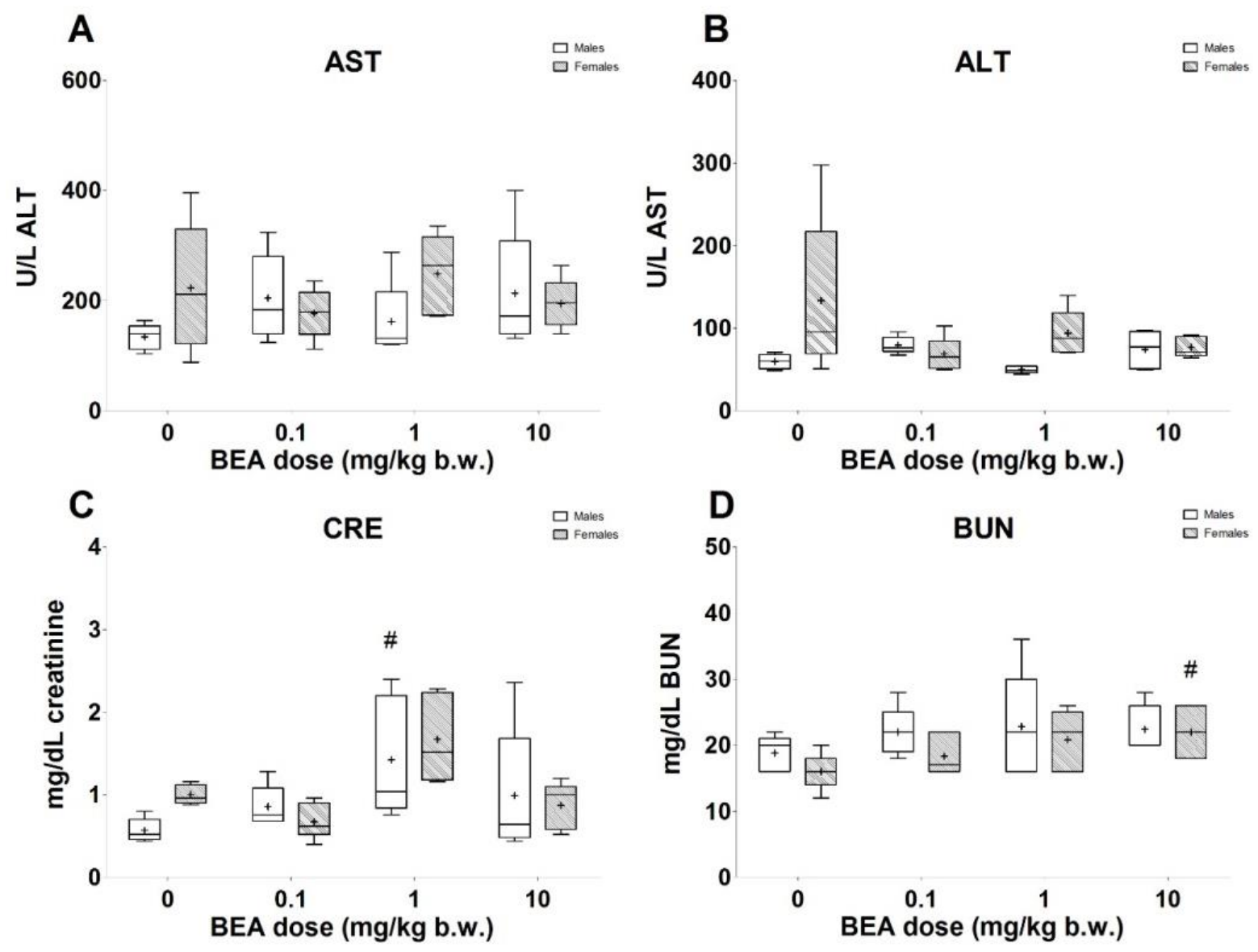

Figure 24: Serum levels of the liver biomarkers aspartate aminotransferase $(A)$ and alanine aminotransferase (B), and of the kidney biomarkers creatinine (C) and blood urea nitrogen (D) in male (white box) and female (striped box) mice orally treated for 42 days with different doses of BEA ( 0 - olive oil; $0.1,1$ and $10 \mathrm{mg} / \mathrm{kg}$ per day). Levels are reported as interquartile box plots, with whiskers delimiting the 10th and 90th percentiles. " + " indicates the mean value for each experimental group. $\# p<0.05$. 


\section{Hormone serum levels}

No treatment-related alterations were present in E2 serum level for both male and female mice (Fig. 25A). In male mice, $T$ levels significantly decreased at BEA10; a significant decrease was observed also in BEA1-treated females (Fig. 25B).

T4 serum levels were significantly increased in male mice at BEA1 and BEA10 (Fig. 25C), whereas TSH was unaffected by BEA treatment (Fig. 25D).
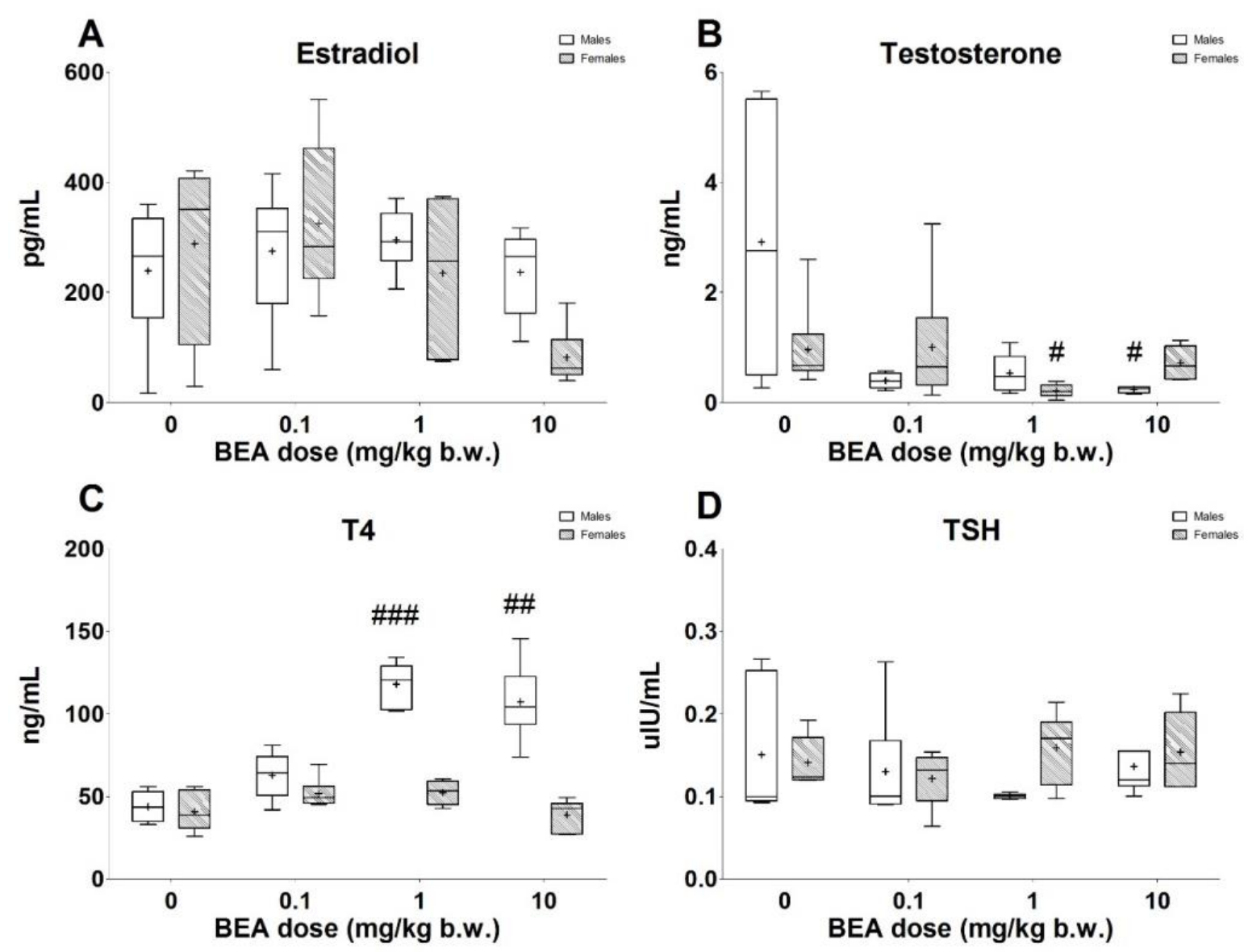

Figure 25: $\quad$ Serum levels of the hormones estradiol (A), testosterone (B), T4 (C) and TSH (D) in male (white box) and female (striped box) mice orally treated for 42 days with different doses of BEA ( 0 - olive oil; $0.1,1$ and $10 \mathrm{mg} / \mathrm{kg}$ per day). Levels are reported as interquartile box plots, with whiskers delimiting the 10th and 90th percentiles. "+" indicates the mean value for each experimental group. \# $p<0.05$; \# $p<0.01$; \#\# $p$ $<0.001$. 


\section{Oxidative stress markers in brain}

The amount of ROS in brain tissues were significantly decreased in male mice at BEA0.1 (Fig. 26A), the correlation with the treatment is unclear. Apparently, at the lowest dose level BEA exerted antioxidant effects. No effect was recorded on GSH brain level in both sexes (Fig. 26B).
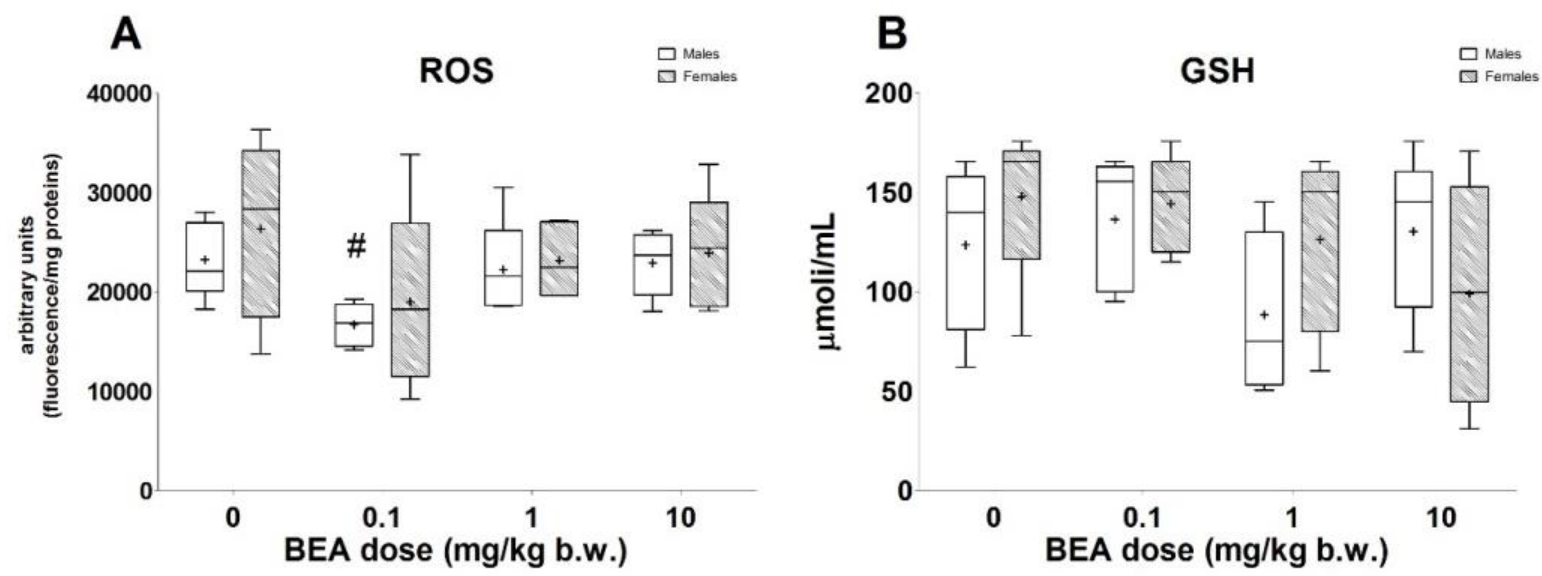

Figure 26: $\quad$ Brain levels of ROS (A) and GSH (B) in male (white box) and female (striped box) mice orally treated for 42 days with different doses of BEA ( 0 - olive oil; $0.1,1$ and 10 $\mathrm{mg} / \mathrm{kg}$ per day). Levels are reported as interquartile box plots, with whiskers delimiting the 10th and 90th percentiles. " + " indicates the mean value for each experimental group. \# $p$ $<0.05$. 


\subsection{4. $\quad$ BEA - Immunotoxicity endpoints}

\section{Lymphoid organ weights}

The results concerning lymphoid organs (spleen, thymus, MLN) were analyzed and reported together with all the organ weights in the "General Toxicity" section (Fig. 15).

\section{Total serum antibodies}

No significant changes were induced by BEA treatment in the antibodies levels in both sexes (Fig. 27A-C).

\section{A}

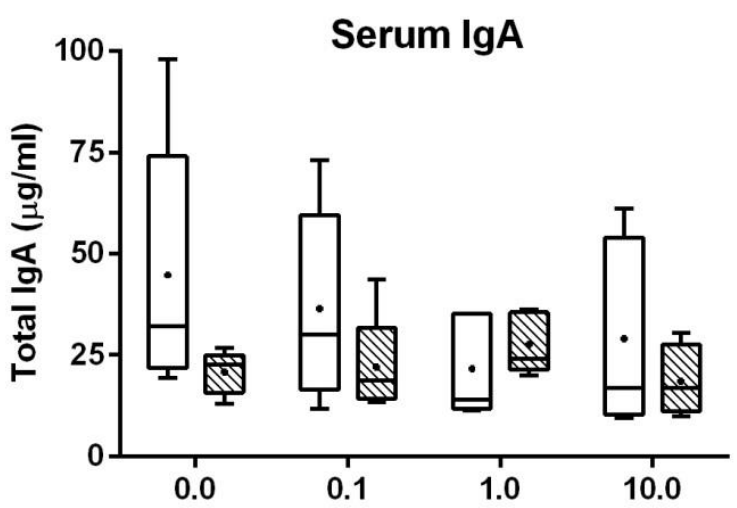

C

BEA dose (mg/kg b.w.)

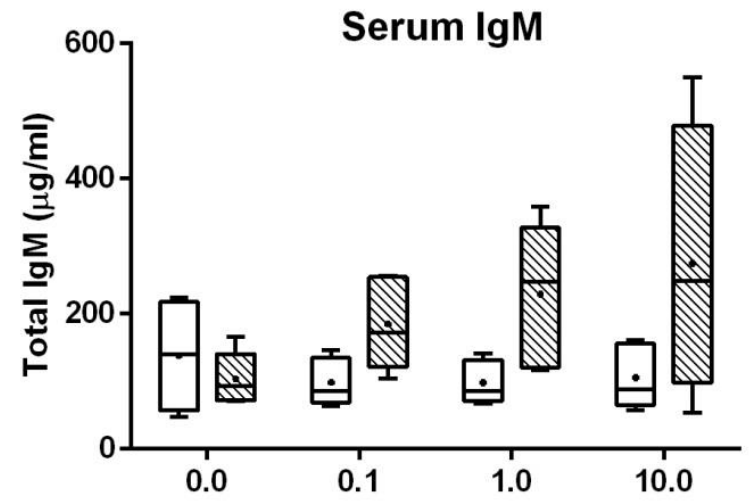

BEA dose (mg/kg b.w.)

Figure 27: Serum antibody levels: total IgA (A), IgG (B) and IgM (C) in male (white box) and female (striped box) mice treated for 42 days orally with different doses of BEA ( 0 - olive oil; $0.1,1$ and $10 \mathrm{mg} / \mathrm{kg}$ per day). Levels are reported as interquartile box plots, with whiskers delimiting the 10 th and 90th percentiles. " + " indicates the mean value for each experimental group. 


\section{Blood count}

The only effect induced by BEA treatment on blood cell populations was observed in the granulocytes (GRAN), which were significantly reduced in male mice at BEA1.0 (Fig. 28). 
$\mathbf{A}$
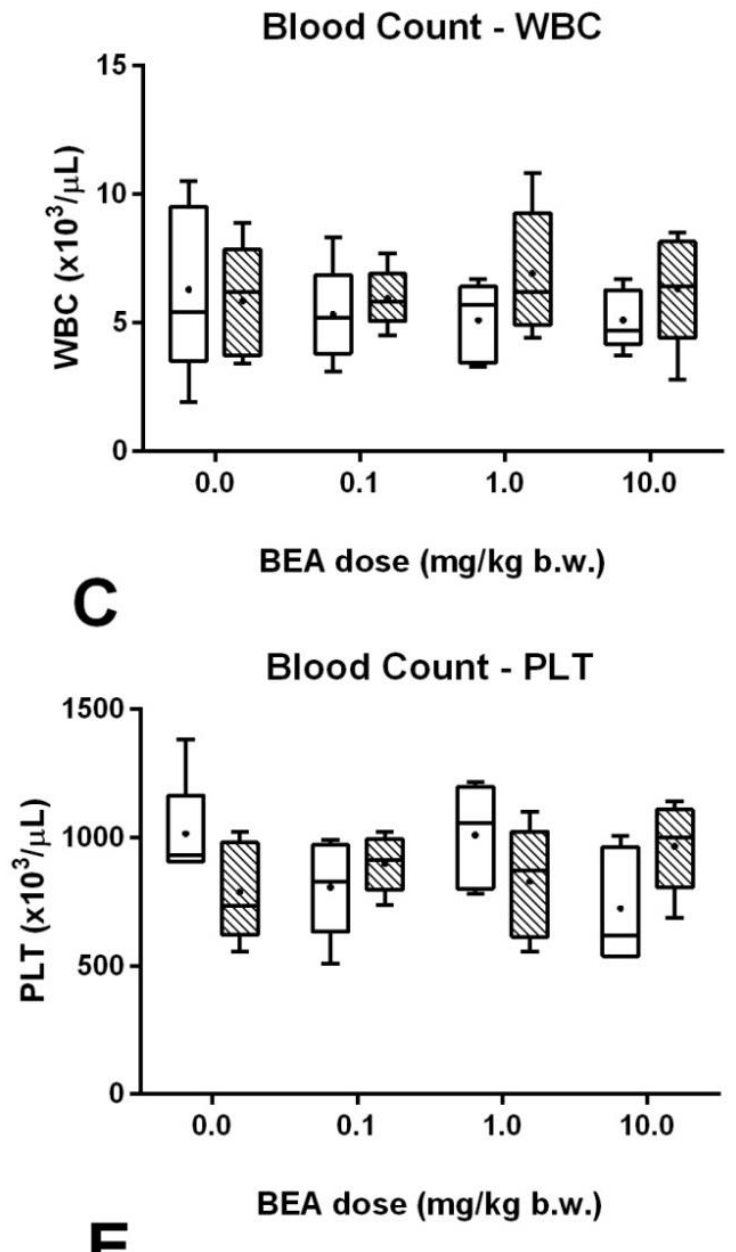

$\mathbf{E}$

Blood Count - LYMPH

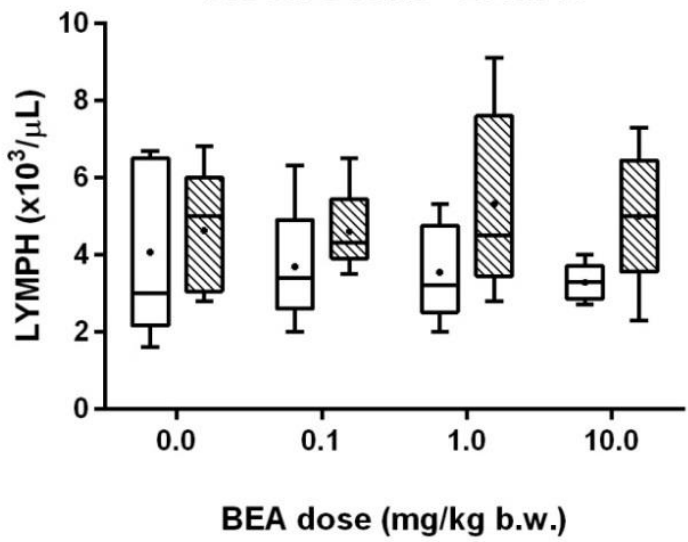

B
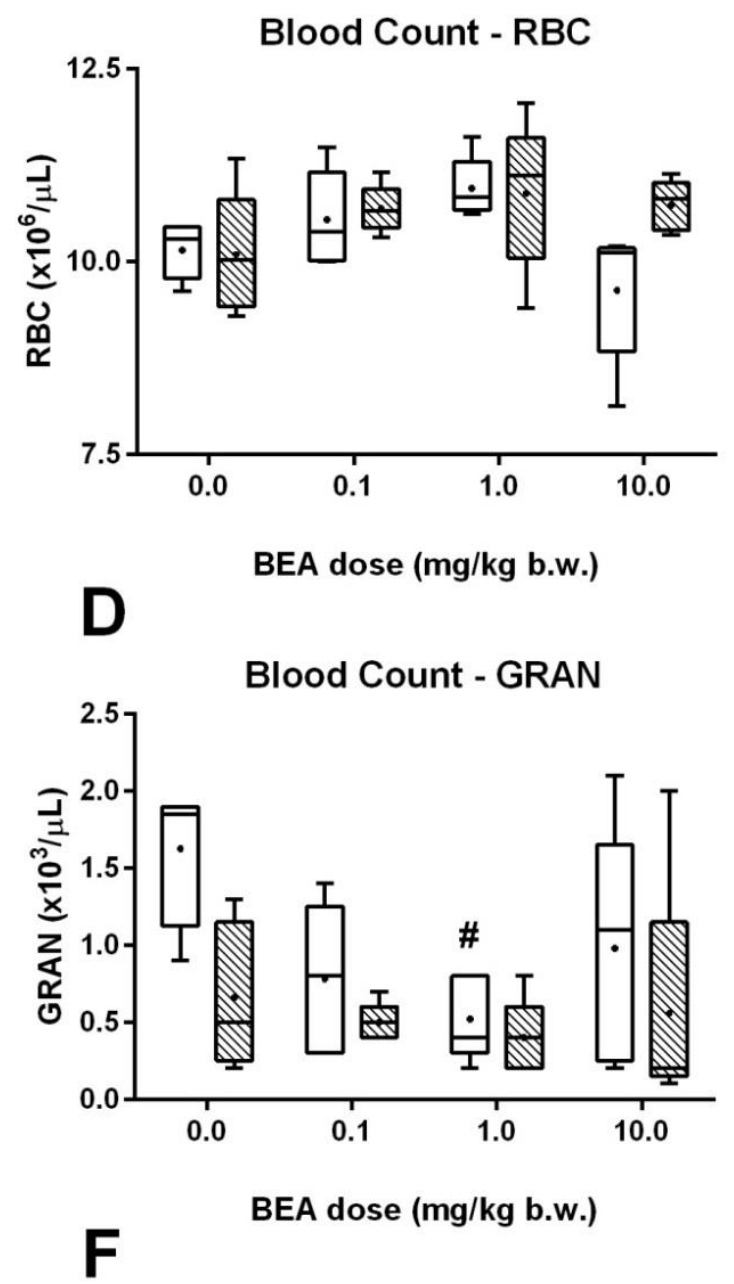

Blood Count - MID

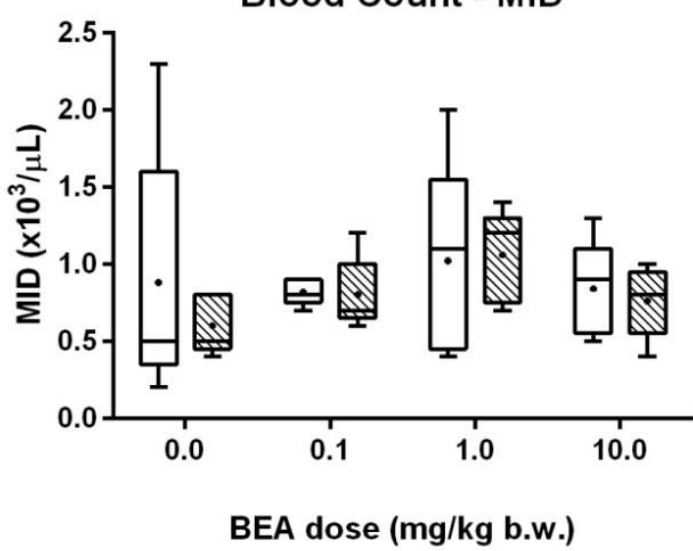

Figure 28: Blood count evaluation: White Blood Cells (WBC, A), Red Blood Cells (RBC, B) and Platelets (PLT, C) levels; among WBC, Granulocytes (GRAN, D), Lymphocytes (LYMPH, E) and rare precursors of white cells (MID, F) in male (white box) and female (striped box) mice orally treated for 42 days with different doses of BEA ( 0 - olive oil; $0.1,1$ and 10 $\mathrm{mg} / \mathrm{kg}$ per day). Levels are reported as interquartile box plots, with whiskers delimiting the 10th and 90th percentiles. " + " indicates the mean value for each experimental group. 


\section{NO production (spleen)}

No spontaneous nitric oxide (NO) production was observed in the absence of LPS stimulation. BEA10 induced opposite effects in female and male mice, significantly reducing or increasing NO levels, respectively (Fig. 29 and Table 19). The higher response to the inflammatory stimulus (LPS) by spleen macrophages in CTRL female versus male mice can be associated to the pro-inflammatory effects of oestrogens through their actions on the innate immune system.

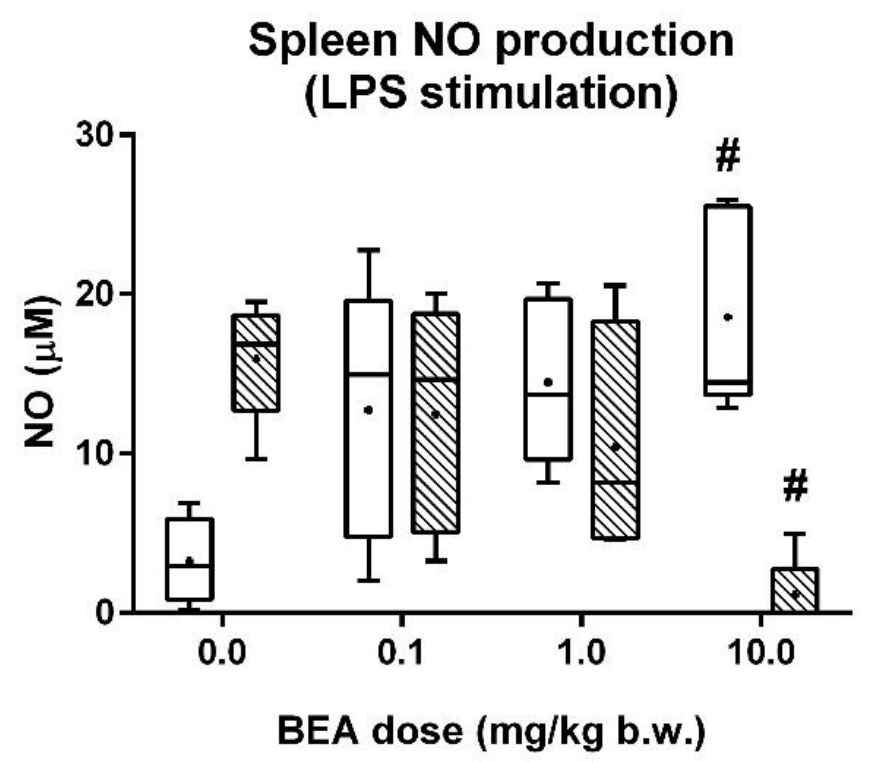

Figure 29: Nitric Oxide (NO) production after in vitro LPS stimulation of adherent spleen cells from male (white box) and female (striped box) mice treated for 42 days orally with different doses of BEA ( 0 - olive oil; $0.1,1$ and $10 \mathrm{mg} / \mathrm{kg}$ per day). NO concentrations in culture supernatants are reported as interquartile box plots, with whiskers delimiting the 10th and 90th percentiles. " + " indicates the mean value for each experimental group. \# $p$ $<0.05$. 
Table 19: Nitric Oxide (NO) production after in vitro LPS stimulation of adherent spleen cells from male and female mice orally treated for 42 days with different doses of BEA $(0.1,1$ and $10 \mathrm{mg} / \mathrm{kg}$ per day).

\begin{tabular}{|c|c|c|c|c|}
\hline & Olive oil & BEA0.1 & BEA1 & BEA10 \\
\hline \multirow[t]{5}{*}{$M A L E$} & 6.88 & 14.93 & 8.19 & 14.47 \\
\hline & 2.94 & 22.77 & 11.04 & 25.12 \\
\hline & 0.14 & 16.41 & 20.67 & 25.92 \\
\hline & 2.94 & 2.01 & 18.70 & 12.87 \\
\hline & & 7.51 & 13.67 & 14.47 \\
\hline$M E A N \pm S D$ & $3.32 \pm 3.39$ & $12.73 \pm 8.08$ & $14.45 \pm 5.20$ & $18.57 \pm 6.38 \#$ \\
\hline \multirow[t]{5}{*}{ FEMALE } & 16.87 & 6.84 & 20.53 & 0.00 \\
\hline & 9.67 & 20.00 & 11.55 & 0.00 \\
\hline & 17.82 & 14.65 & 4.81 & 0.00 \\
\hline & 15.73 & 17.55 & 4.63 & 0.56 \\
\hline & 19.52 & 3.26 & & 4.97 \\
\hline$M E A N \pm S D$ & $15.92 \pm 3.76$ & $12.46 \pm 7.14$ & $10.38 \pm 7.45$ & $1.11 \pm 2.17 \#$ \\
\hline
\end{tabular}

Values are reported as $\mu \mathrm{M}$ concentration of $\mathrm{NO}$ in culture supernatants. \# $\mathrm{p}<0.05$

\section{Cytokine production (spleen)}

No spontaneous cytokine production was observed in the absence of stimulation. After BEA treatment, no significant differences in both cytokine levels were observed in male mice, whereas in female mice treatment with the BEA10 and BEA0.1 induced significant increase of IFNY and IL-10 production, respectively (Fig. 30).
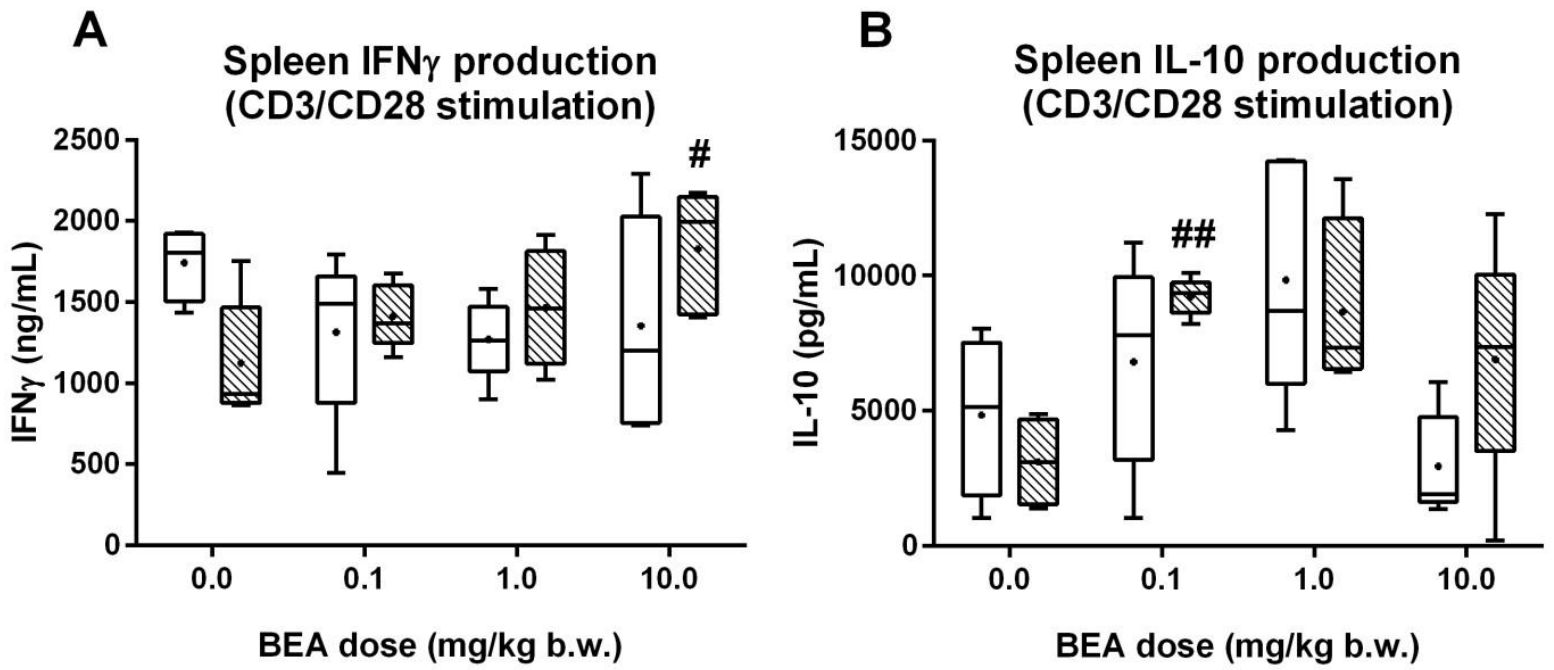

Figure 30: Interferon- $\mathrm{Y}$ (IFNY, A) and Interleukin-10 (IL-10, B) production after in vitro CD3/CD28 stimulation of spleen cells from male (white box) and female (striped box) mice orally treated for 42 days with different doses of BEA ( 0 - olive oil; $0.1,1$ and $10 \mathrm{mg} / \mathrm{kg}$ per day). Cytokine concentrations in culture supernatants are as interquartile box plots, with whiskers delimiting the 10th and 90th percentiles. "+" indicates the mean value for each experimental group. \# $\mathrm{p}<0.05 ; \# \# \mathrm{p}<0.01$. 


\section{T lymphocyte (sub) populations (spleen)}

BEA treatment induced only in female mice significant increase in the number and frequency of CD3+ lymphocytes (whole T lymphocytes), and in the number of double positive CD3+/CD4+ lymphocytes (helper T lymphocytes), at BEA0.1 (Fig. 31). 
A

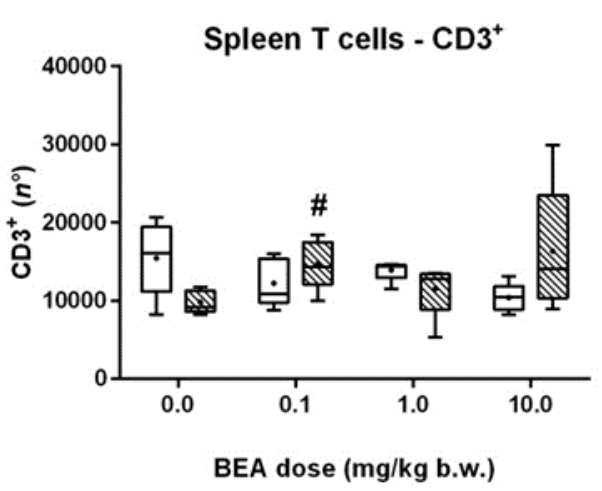

C
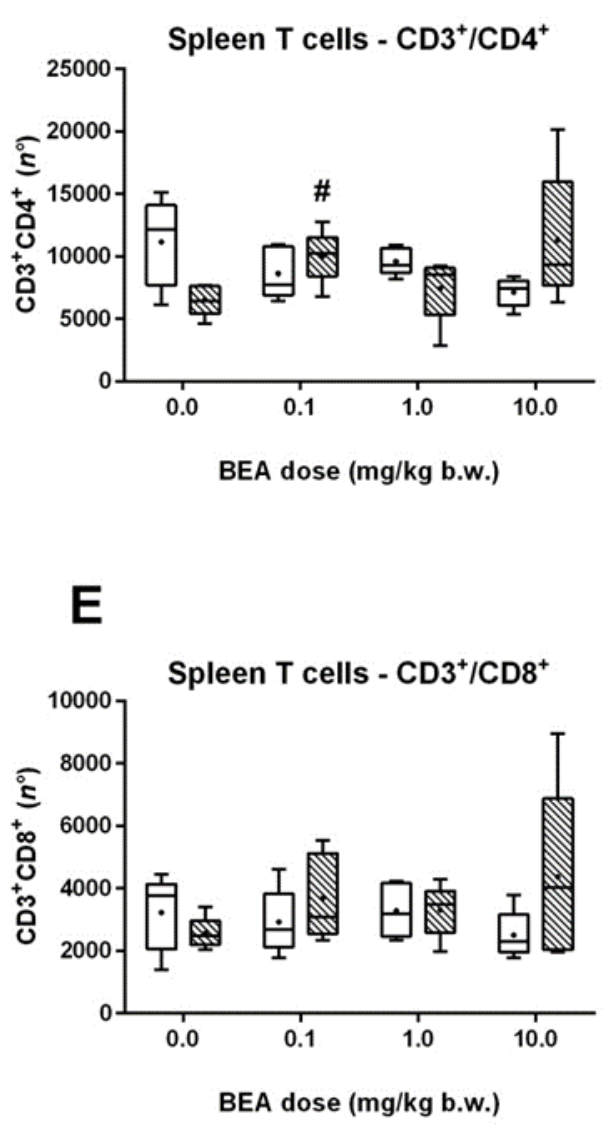

B

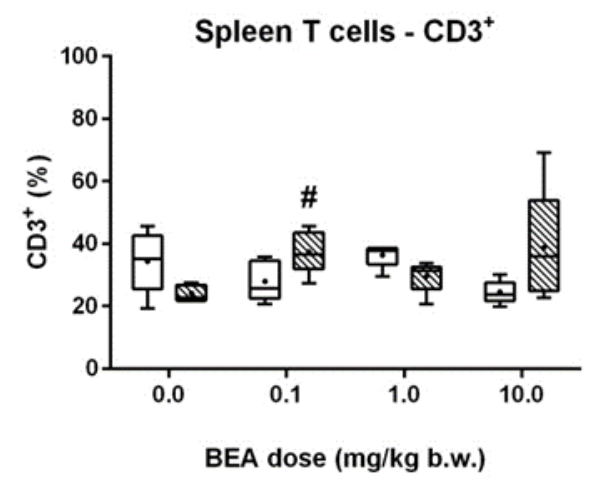

D

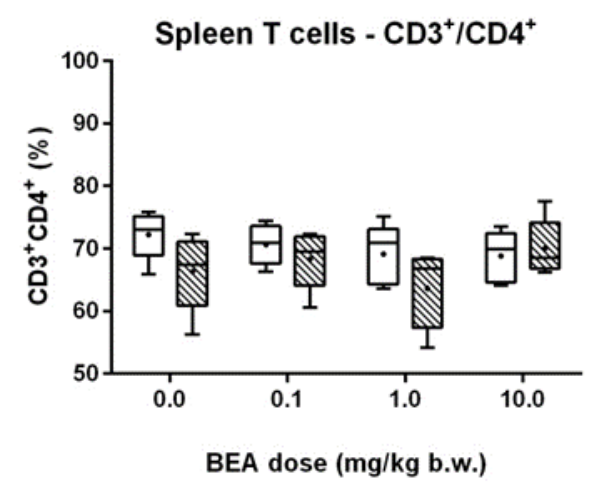

$\mathbf{F}$

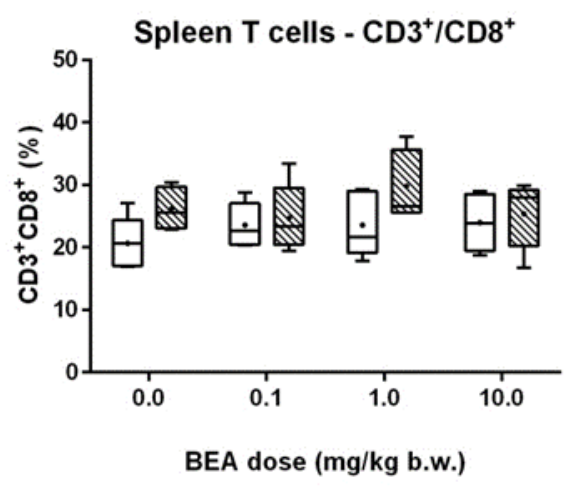

Figure 31: T cell subpopulations in spleens from male (white box) and female (striped box) mice orally treated for 42 days with different doses of BEA ( 0 - olive oil; $0.1,1$ and $10 \mathrm{mg} / \mathrm{kg}$ per day), evaluated by cytofluorimetric phenotype analysis. Number and percentage of CD3-positive T cells ( $\left.n^{\circ} \mathrm{CD} 3+, A ; \% C D 3+, B\right), C D 3 / C D 4-d o u b l e ~ p o s i t i v e ~ T$ cells $\left(n^{\circ}\right.$ $\mathrm{CD} 3+/ \mathrm{CD} 4+, \mathrm{C}$; \%CD3+/CD4+, D), CD3/CD8-double positive $T$ cells $\left(\mathrm{n}^{\circ} \mathrm{CD} 3+/ \mathrm{CD} 8+, \mathrm{E}\right.$; $\% \mathrm{CD} 3+/ \mathrm{CD} 8+, \mathrm{F})$, are reported as interquartile box plots, with whiskers delimiting the 10th and 90th percentiles. " + " indicates the mean value for each experimental group. \# $p$ $<0.05$. 


\subsection{5. $\quad$ BEA - Genotoxicity endpoints}

\section{Comet assay}

The complete set of parameters collected on all organs analyzed by alkaline Comet in male mice are reported in Table 20. In the same Table also results obtained in the negative and positive CTRL groups are reported. MMS treatment, used as a positive CTRL, induced a significant increase of all parameters of DNA damage over those observed in the CTRL group in all analyzed organs.

Table 20: Alkaline Comet assay in different organs/tissues of male mice after repeated oral treatments with different doses of BEA ( 0 - olive oil; $0.1,1$ and $10 \mathrm{mg} / \mathrm{kg}$ per day) or MMS as positive CTRL. For each experimental group the mean of median and standard error of the values obtained from each animal for tail length (Tail-L), tail intensity (\%TI) and Olive Tail Moment (OTM) are reported. The mean \% hedgehogs for each group is shown.

\begin{tabular}{|c|c|c|c|c|c|}
\hline Tissue/organ & Treatment & $\begin{array}{c}\text { Tail-L } \\
\text { mean } \pm \text { SE }\end{array}$ & $\begin{array}{c}\% \text { TI } \\
\text { mean } \pm \text { SE }\end{array}$ & $\begin{array}{c}\mathrm{OTM} \\
\text { mean } \pm \mathrm{SE}\end{array}$ & $\begin{array}{c}\% \text { hedgehogs } \\
\text { mean } \pm \text { SE }\end{array}$ \\
\hline \multirow[t]{5}{*}{ Blood } & olive oil & $0.370 \pm 0.118$ & $0.172 \pm 0.018$ & $0.008 \pm 0.003$ & $0.105 \pm 0.105$ \\
\hline & BEAO.1 & $0.678 \pm 0.225$ & $0.206 \pm 0.032$ & $0.021 \pm 0.011$ & $0.118 \pm 0.118$ \\
\hline & BEA1 & $0.339 \pm 0.166$ & $0.162 \pm 0.012$ & $0.010 \pm 0.006$ & $0.235 \pm 0.144$ \\
\hline & BEA10 & $0.210 \pm 0.048$ & $0.144 \pm 0.006$ & $0.005 \pm 0.001$ & $0.228 \pm 0.140$ \\
\hline & MMS & $18.270 \pm 1.809 \# \#$ & $15.189 \pm 3.127 \# \#$ & $8.474 \pm 2.069 \# \#$ & $41.014 \pm 5.543 \# \# \#$ \\
\hline \multirow[t]{5}{*}{ Liver } & olive oil & $0.549 \pm 0.150$ & $0.103 \pm 0.009$ & $0.010 \pm 0.003$ & $3.159 \pm 1.339$ \\
\hline & BEA0.1 & $1.403 \pm 0.352$ & $0.194 \pm 0.031$ & $0.046 \pm 0.017$ & $4.848 \pm 1.241$ \\
\hline & BEA1 & $0.758 \pm 0.385$ & $0.126 \pm 0.041$ & $0.021 \pm 0.013$ & $2.276 \pm 0.919$ \\
\hline & BEA10 & $0.887 \pm 0.629$ & $0.138 \pm 0.079$ & $0.036 \pm 0.034$ & $2.173 \pm 0.572$ \\
\hline & MMS & $32.250 \pm 3.523 \# \# \#$ & $25.765 \pm 5.808 \# \#$ & $21.939 \pm 5.429 \# \#$ & $3.411 \pm 0.536$ \\
\hline \multirow[t]{5}{*}{ Kidney } & olive oil & $1.193 \pm 0.220$ & $0.244 \pm 0.019$ & $0.049 \pm 0.010$ & $2.829 \pm 0.800$ \\
\hline & BEA0.1 & $1.629 \pm 0.253$ & $0.314 \pm 0.043$ & $0.076 \pm 0.023$ & $3.081 \pm 0.710$ \\
\hline & BEA1 & $2.983 \pm 0.667 \#$ & $0.682 \pm 0.182 \#$ & $0.216 \pm 0.069 \#$ & $4.089 \pm 0.495$ \\
\hline & BEA10 & $1.065 \pm 0.069$ & $0.173 \pm 0.019$ & $0.032 \pm 0.004$ & $2.877 \pm 0.858$ \\
\hline & MMS & $29.977 \pm 5.229 \# \# \#$ & $28.259 \pm 7.202 \# \#$ & $19.014 \pm 5.834 \# \#$ & $3.780 \pm 1.785$ \\
\hline \multirow[t]{5}{*}{ Duodenum } & olive oil & $0.549 \pm 0.181$ & $0.109 \pm 0.016$ & $0.011 \pm 0.004$ & $5.807 \pm 0.330$ \\
\hline & BEA0.1 & $0.339 \pm 0.181$ & $0.128 \pm 0.016$ & $0.009 \pm 0.004$ & $9.088 \pm 1.585$ \\
\hline & BEA1 & $1.387 \pm 0.188 \#$ & $0.337 \pm 0.065 \# \#$ & $0.058 \pm 0.013 \#$ & $8.513 \pm 1.177$ \\
\hline & BEA10 & $0.807 \pm 0.209$ & $0.172 \pm 0.026$ & $0.033 \pm 0.015$ & $6.756 \pm 1.313$ \\
\hline & MMS & $22.543 \pm 4.649 \# \# \#$ & $14.599 \pm 4.312 \# \#$ & $9.154 \pm 3.273 \#$ & $9.963 \pm 1.477 \#$ \\
\hline \multirow[t]{5}{*}{ Testis } & olive oil & $0.162 \pm 0.044$ & $0.220 \pm 0.029$ & $0.001 \pm 0.000$ & $1.102 \pm 0.556$ \\
\hline & BEA0.1 & $0.242 \pm 0.114$ & $0.182 \pm 0.019$ & $0.007 \pm 0.003$ & $0.727 \pm 0.120$ \\
\hline & BEA1 & $0.468 \pm 0.170$ & $0.149 \pm 0.014$ & $0.010 \pm 0.005$ & $2.703 \pm 0.501$ \\
\hline & BEA10 & $0.129 \pm 0.060$ & $0.169 \pm 0.011$ & $0.002 \pm 0.001$ & $1.877 \pm 0.603$ \\
\hline & MMS & $8.949 \pm 1.737 \# \# \#$ & $5.478 \pm 1.754 \# \#$ & $2.316 \pm 0.761 \# \#$ & $1.542 \pm 0.491$ \\
\hline
\end{tabular}

$\# p<0.05 ; \# \# p<0.01 ; \# \# \# p<0.001$

Figure 32 summarizes the Comet assay results collected in the same organs, expressed by tail intensity values (left). In the same figure, the percentage of hedgehogs is also reported (right). Treatment with BEA did not significantly increase the \%TI in blood leukocytes, liver and testis cells, whereas a statistically significant increase was detected for kidney and duodenum cells from mice 
treated with the intermediate BEA dose (BEA1; $1 \mathrm{mg} / \mathrm{kg}$ b.w.). In kidney, at this dose, the single animal \%TI values were more heterogeneous than in the other groups, however, values of 4 out of 5 BEA1-treated animals were higher than the highest value measured in CTRL animals. Since the heterogeneity was not observed in other organs of the same animals the effects in kidney and duodenum appear related to the treatment and not to an experimental bug. The percentage of hedgehogs was rather low in all the organs analyzed, and also in duodenum, the tissue with the highest values, this percentage remained below $15 \%$. Treatment with BEA did not induce an increase of these highly damaged cells. 
A
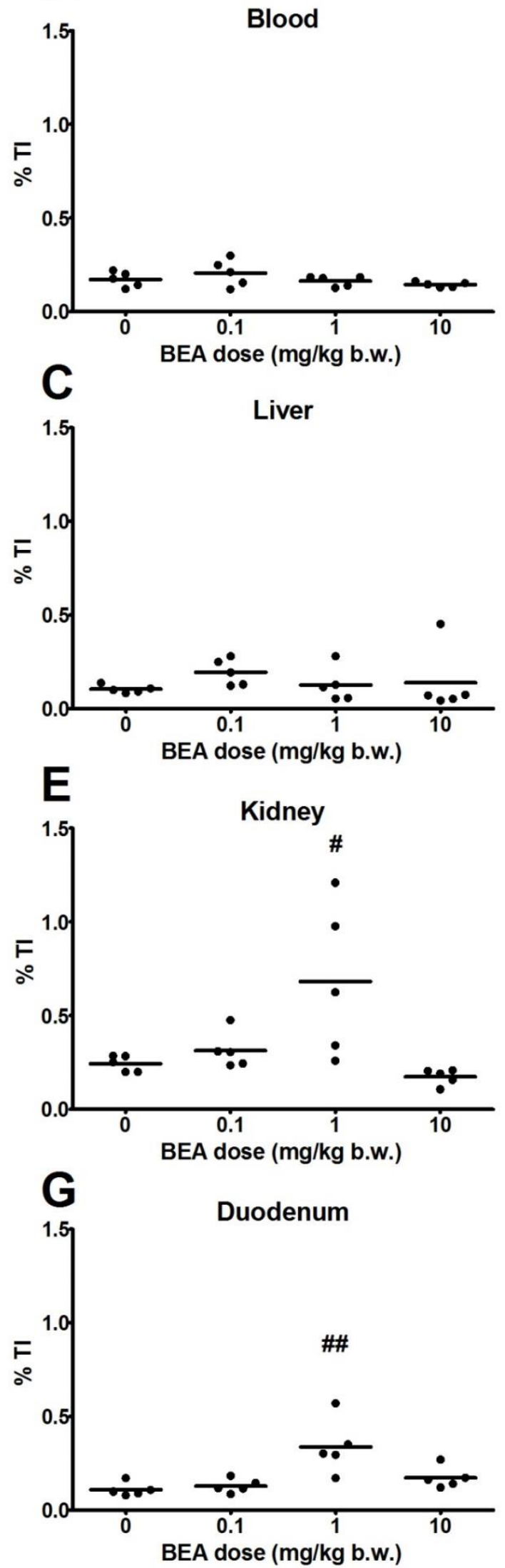

B

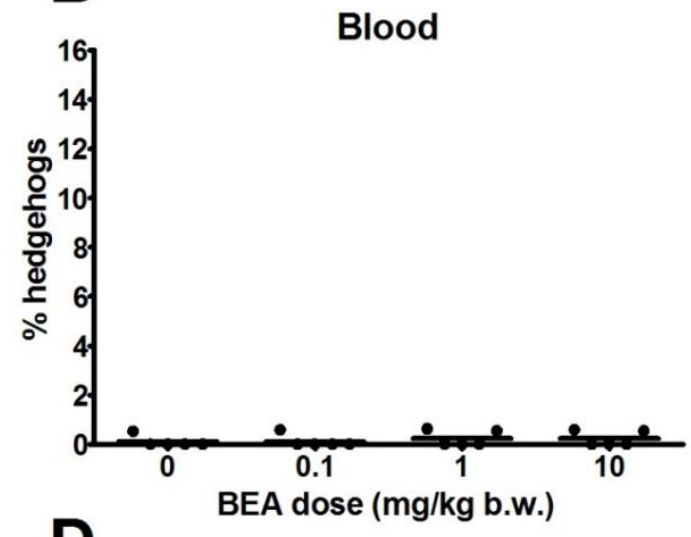

Liver
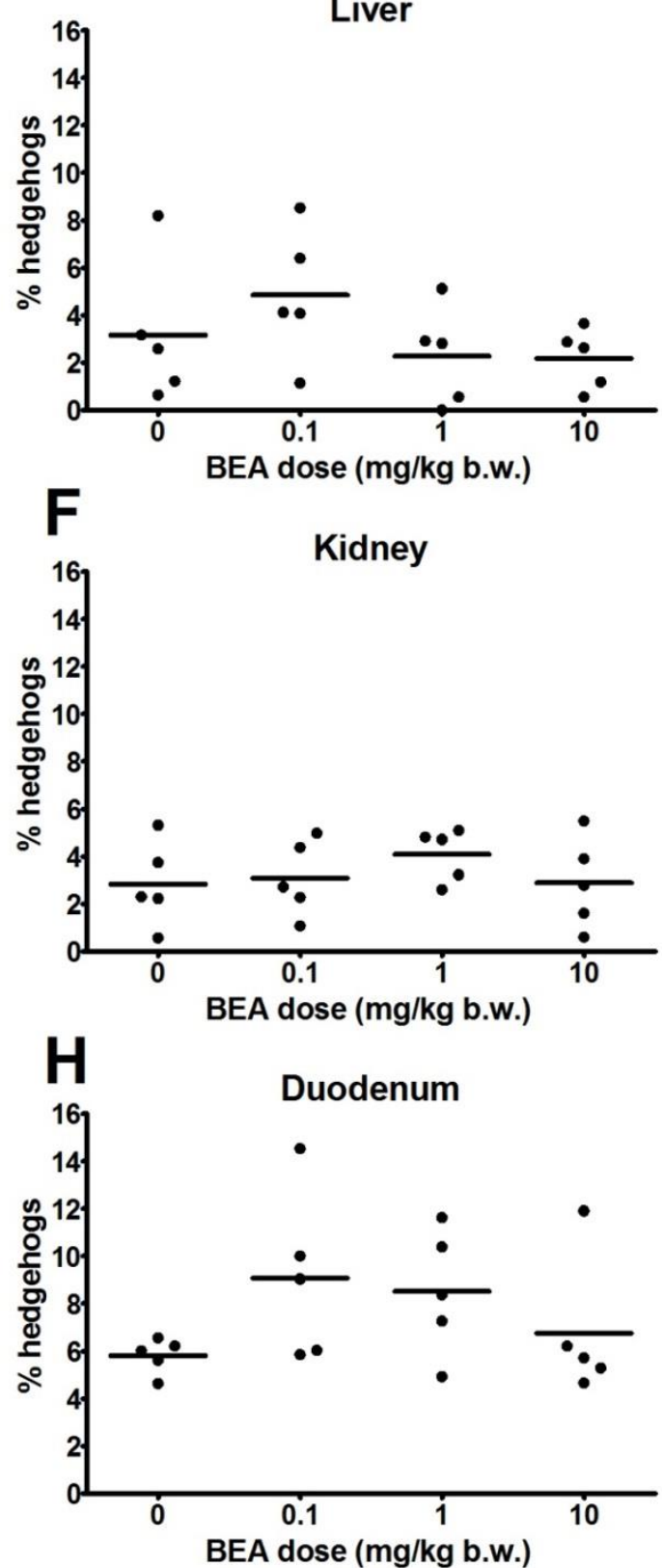

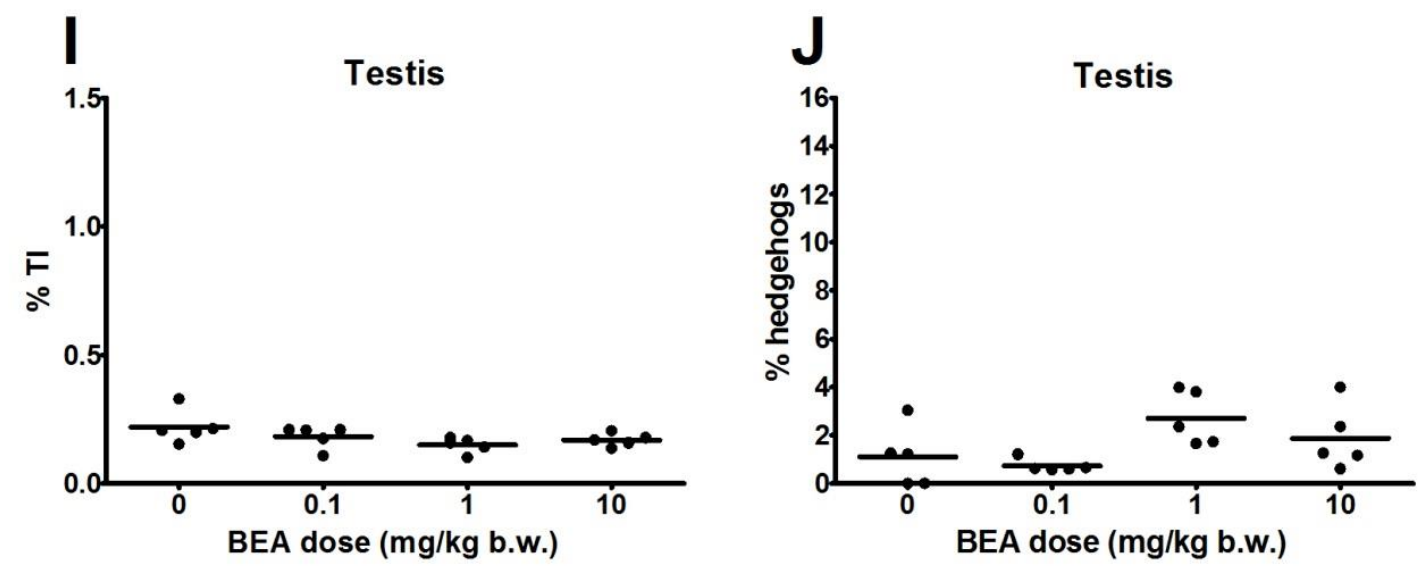

Figure 32: $\quad$ Alkaline Comet assay in different organs/tissues of male mice after repeated oral treatments with different doses of BEA ( 0 - olive oil; $0.1,1$ and $10 \mathrm{mg} / \mathrm{kg}$ per day). In A, C, E, G and I dots represent the median of tail intensity obtained for each animal and lines represent the mean of tail intensity for each group. In $B, D, F, H$ and $J$ dots represent the percentage of hedgehogs obtained for each mouse and lines are the mean for each group. \# $\mathrm{p}<0.05 ; \#$; $<0.01$.

Epididymal spermatozoa were analyzed by neutral Comet assay and results are reported in Figure 33. BEA treatment did not induce any increase of \%TI.

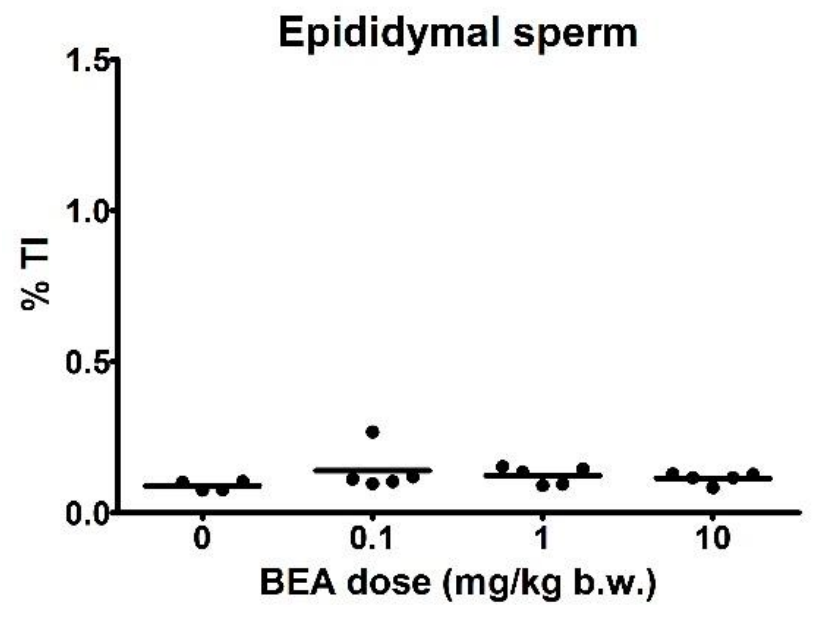

Figure 33: Neutral Comet assay in epididymal spermatozoa of male mice after repeated oral treatments with different doses of BEA ( 0 - olive oil; $0.1,1$ and $10 \mathrm{mg} / \mathrm{kg}$ per day). Dots represent the median of tail intensity obtained for each animal and lines represent the mean of tail intensity for each group.

Results obtained in female mice are shown in Table 21 and Figure 34. In all observed parameters there is not a statistically significant difference except for tail length which was increased by treatment with BEA10 $\mathrm{mg} / \mathrm{kg}$ in ovary. MMS treatment used as a positive CTRL, induced a significant increase of tail length, \%TI and OTM over CTRL values in blood, liver, kidney and duodenum; in ovary MMS induced an increase of Comet parameters, however, due to the high variability among animals, a significant increase was only achieved for tail length values. MMS treatment induced a significant increase of \% hedgehogs only in blood. 
Table 21: Alkaline Comet assay in different organs/tissues of female mice after repeated oral treatments with different doses of BEA ( 0 - olive oil; $0.1,1$ and $10 \mathrm{mg} / \mathrm{kg}$ per day) or MMS as positive CTRL. For each experimental group the mean of median and standard error of the values obtained from each animal for tail length (Tail-L), tail intensity (\%TI) and Olive Tail Moment (OTM) are reported. The mean \% hedgehogs for each group is shown.

\begin{tabular}{|c|c|c|c|c|c|}
\hline Tissue/organ & Treatment & $\begin{array}{c}\text { Tail-L } \\
\text { mean } \pm \text { SE }\end{array}$ & $\begin{array}{c}\% \text { \%TI } \\
\text { mean } \pm \text { SE }\end{array}$ & $\begin{array}{c}\text { OTM } \\
\text { mean } \pm \text { SE }\end{array}$ & $\begin{array}{c}\% \text { hedgehogs } \\
\text { mean } \pm \text { SE }\end{array}$ \\
\hline \multirow[t]{5}{*}{ Blood } & olive oil & $0.006 \pm 0.006$ & $0.057 \pm 0.006$ & $0.008 \pm 0.001$ & $0.000 \pm 0.000$ \\
\hline & BEA0.1 & $0.698 \pm 0.410$ & $0.216 \pm 0.097$ & $0.022 \pm 0.009$ & $0.000 \pm 0.000$ \\
\hline & BEA1 & $0.256 \pm 0.200$ & $0.089 \pm 0.028$ & $0.011 \pm 0.002$ & $0.000 \pm 0.000$ \\
\hline & BEA10 & $0.574 \pm 0.078$ & $0.147 \pm 0.013$ & $0.016 \pm 0.002$ & $0.000 \pm 0.000$ \\
\hline & MMS & $21.232 \pm 3.947 \# \# \#$ & $25.732 \pm 7.387 \# \#$ & $4.480 \pm 1.471 \#$ & $11.740 \pm 4.785 \#$ \\
\hline \multirow[t]{5}{*}{ Liver } & olive oil & $0.767 \pm 0.204$ & $0.163 \pm 0.025$ & $0.015 \pm 0.003$ & $2.133 \pm 0.904$ \\
\hline & BEA0.1 & $1.426 \pm 0.602$ & $0.346 \pm 0.177$ & $0.038 \pm 0.021$ & $1.336 \pm 0.666$ \\
\hline & BEA1 & $0.652 \pm 0.096$ & $0.131 \pm 0.0190$ & $0.015 \pm 0.002$ & $2.670 \pm 0.942$ \\
\hline & BEA10 & $0.337 \pm 0.059$ & $0.092 \pm 0.013$ & $0.010 \pm 0.001$ & $1.735 \pm 0.500$ \\
\hline & MMS & $27.558 \pm 9.128 \# \# \#$ & $39.026 \pm 10.276 \# \#$ & $8.408 \pm 2.650 \#$ & $13.732 \pm 7.505$ \\
\hline \multirow[t]{5}{*}{ Kidney } & olive oil & $1.023 \pm 0.196$ & $0.281 \pm 0.039$ & $0.027 \pm 0.004$ & $1.733 \pm 0.340$ \\
\hline & BEA0.1 & $1.559 \pm 0.629$ & $0.521 \pm 0.245$ & $0.054 \pm 0.027$ & $0.933 \pm 0.340$ \\
\hline & BEA1 & $0.350 \pm 0.133$ & $0.134 \pm 0.016$ & $0.012 \pm 0.001$ & $0.400 \pm 0.267$ \\
\hline & BEA10 & $0.325 \pm 0.044$ & $0.100 \pm 0.002$ & $0.010 \pm 0.000$ & $2.133 \pm 0.929$ \\
\hline & MMS & $22.625 \pm 2.442 \# \# \#$ & $28.825 \pm 7.332 \# \#$ & $5.464 \pm 1.627 \#$ & $3.100 \pm 1.089$ \\
\hline \multirow[t]{5}{*}{ Duodenum } & olive oil & $1.234 \pm 0.252$ & $0.700 \pm 0.145$ & $0.064 \pm 0.012$ & $3.739 \pm 1.165$ \\
\hline & BEA0.1 & $0.665 \pm 0.117$ & $0.609 \pm 0.096$ & $0.055 \pm 0.011$ & $2.002 \pm 0.559$ \\
\hline & BEA1 & $1.116 \pm 0.284$ & $1.358 \pm 0.571$ & $0.123 \pm 0.054$ & $2.811 \pm 1.222$ \\
\hline & BEA10 & $0.618 \pm 0.113$ & $0.406 \pm 0.076$ & $0.035 \pm 0.006$ & $4.002 \pm 0.943$ \\
\hline & MMS & $18.778 \pm 3.304 \# \# \#$ & $25.610 \pm 5.657 \# \#$ & $4.470 \pm 1.263 \# \#$ & $7.394 \pm 5.262$ \\
\hline \multirow[t]{5}{*}{ Ovary } & olive oil & $0.233 \pm 0.075$ & $0.231 \pm 0.043$ & $0.018 \pm 0.003$ & $0.950 \pm 0.594$ \\
\hline & BEA0.1 & $0.420 \pm 0.183$ & $0.199 \pm 0.025$ & $0.019 \pm 0.003$ & $2.667 \pm 0.596$ \\
\hline & BEA1 & $0.513 \pm 0.126$ & $0.189 \pm 0.038$ & $0.019 \pm 0.004$ & $1.733 \pm 0.909$ \\
\hline & BEA10 & $0.908 \pm 0.251 \#$ & $0.321 \pm 0.054$ & $0.029 \pm 0.007$ & $3.385 \pm 1.065$ \\
\hline & MMS & $18.044 \pm 7.455 \#$ & $15.799 \pm 7.706$ & $3.320 \pm 2.177$ & $1.000 \pm 0.652$ \\
\hline
\end{tabular}

$\# p<0.05 ; \# \# p<0.01 ; \# \# \# p<0.001$. 

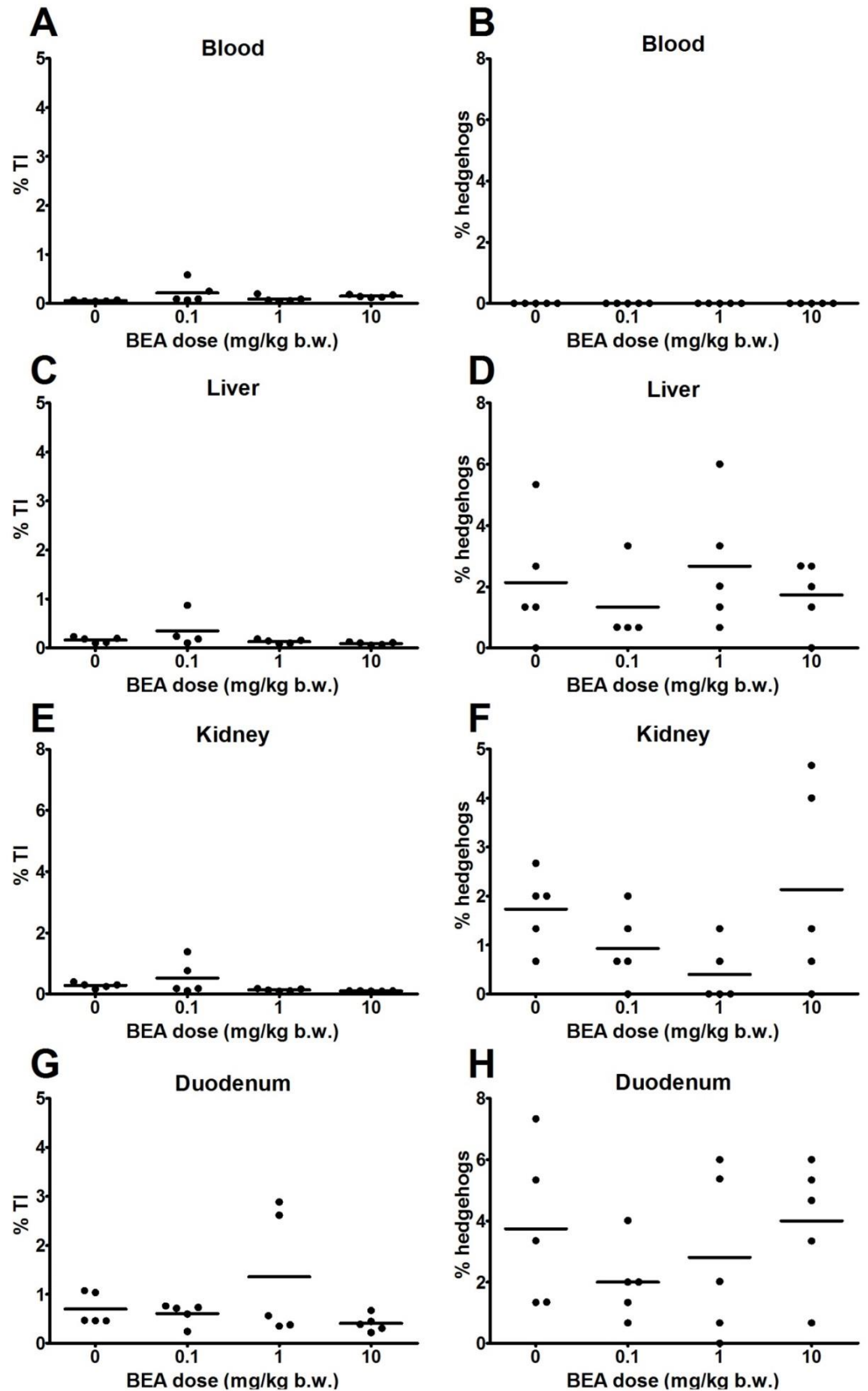

The present document has been produced and adopted by the bodies identified above as authors. In accordance with Article 36 of Regulation (EC) No $178 / 2002$, this task has been carried out exclusively by the authors in the context of a grant agreement between the European Food Safety Authority and the authors. The present document is published complying with the transparency principle to which the Authority is subject. It cannot be considered as an output adopted by the Authority. The European Food Safety Authority reserves its rights, view and position as regards the issues addressed and the conclusions reached in the present document, without prejudice to the rights of the authors. 

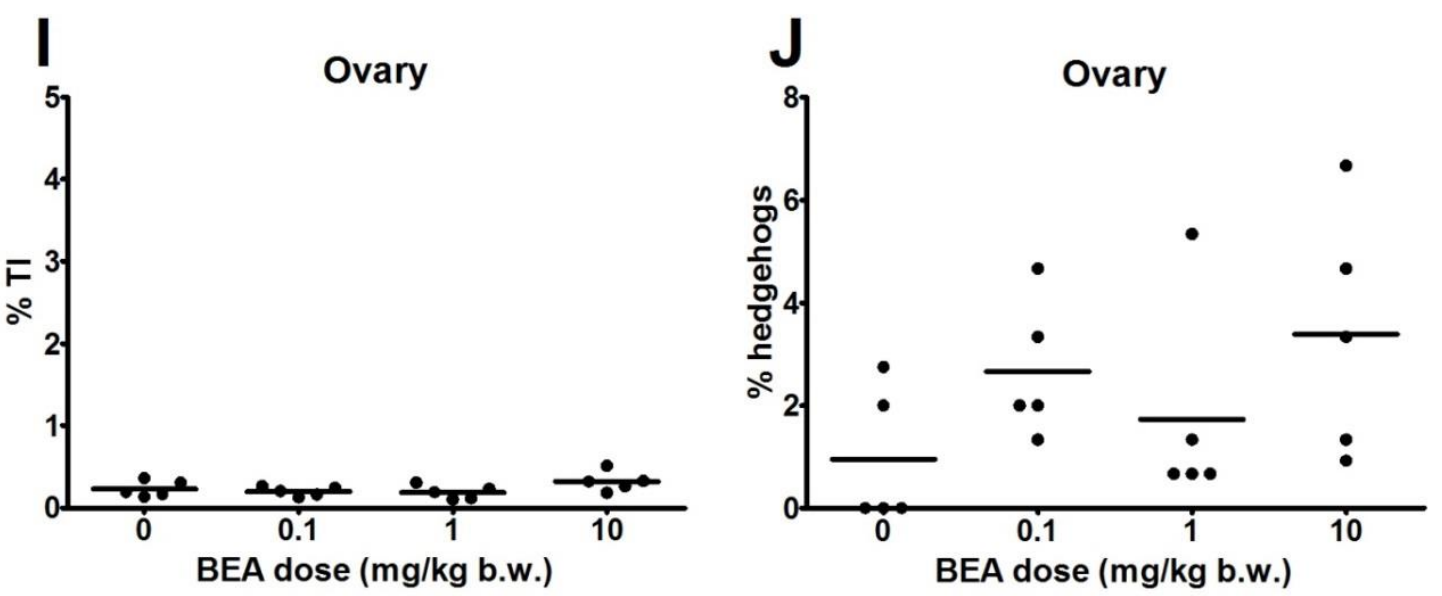

Figure 34: $\quad$ Alkaline Comet assay in different organs/tissues of female mice after repeated oral treatments with different doses of BEA ( 0 - olive oil; $0.1,1$ and $10 \mathrm{mg} / \mathrm{kg}$ per day). In A, $C, E, G$ and I dots represent the median of tail intensity obtained for each animal and lines represent the mean of tail intensity for each group. In $\mathrm{B}, \mathrm{D}, \mathrm{F}, \mathrm{H}$ and $\mathrm{J}$ dots represent the percentage of hedgehogs obtained for each mouse and lines are the mean for each group.

Considering these results, the compound cannot be considered clearly positive since not all the criteria listed in OECD TG $489^{1}$ are fulfilled. In particular, there is no dose-related increase. Moreover the increase of \%TI in kidney and duodenum after $1 \mathrm{mg} / \mathrm{kg}$ BEA was only observed in males and not in females and dams (see also paragraph 3.4.4). As far as the effects of $1 \mathrm{mg} / \mathrm{kg}$ BEA on kidney of male mice is concerned, the reduction of the organ weight (Fig. 15; Table 17) and the creatinine increase (Fig. 24) suggest a slight toxic effect of the compound which may also be involved in the increase in DNA migration.

\section{Pig-a assay}

The complete set of parameters obtained in male mice are reported in Table 22. In the same table also results of the negative and positive CTRL groups are reported. ENU treatment, used as a positive CTRL, induced a significant increase of both mutant RETs and RBCs. No increase of mutant cells and no induction of toxicity were observed after exposure to BEA as also shown in Figure 35 . These results indicate that BEA does not induce gene mutations in bone marrow precursors of red blood cells.

\footnotetext{
${ }^{1}$ OECD, Test No. 489, 2014: In Vivo Mammalian Alkaline Comet Assay, OECD Publishing, Paris.
} 
Table 22: Pig-a assays in male mice after repeated oral treatments with different doses of BEA ( 0 - olive oil; $0.1,1$ and $10 \mathrm{mg} / \mathrm{kg}$ per day).

\begin{tabular}{lccc}
\hline Treatment & $\begin{array}{c}\text { \%RETs } \\
\text { mean } \pm \text { SE }\end{array}$ & $\begin{array}{c}\text { Mutant RBCs per } \\
\mathbf{1 0}^{6} \text { Total RBCs } \\
\text { mean } \pm \text { SE }\end{array}$ & $\begin{array}{c}\text { Mutant RETs per 10 } \\
\text { Total RETs } \\
\text { mean } \pm \text { SE }\end{array}$ \\
\hline olive oil & $2.920 \pm 0.250$ & $1.000 \pm 0.800$ & $0.400 \pm 0.100$ \\
BEA0.1 & $3.260 \pm 0.300$ & $0.500 \pm 0.170$ & $0.800 \pm 0.460$ \\
BEA1 & $2.700 \pm 0.160$ & $0.160 \pm 0.050$ & $0.460 \pm 0.340$ \\
BEA10 & $2.960 \pm 0.140$ & $0.220 \pm 0.080$ & $0.680 \pm 0.552$ \\
ENU & $2.700 \pm 0.227$ & $15.950 \pm 1.184 \# \# \#$ & $79.300 \pm 13.839 \# \# \#$ \\
\hline
\end{tabular}

\#\#\# $\mathrm{p}<0.001 . ;{ }^{\mathrm{a}}$ One ENU treated animal died before blood collection
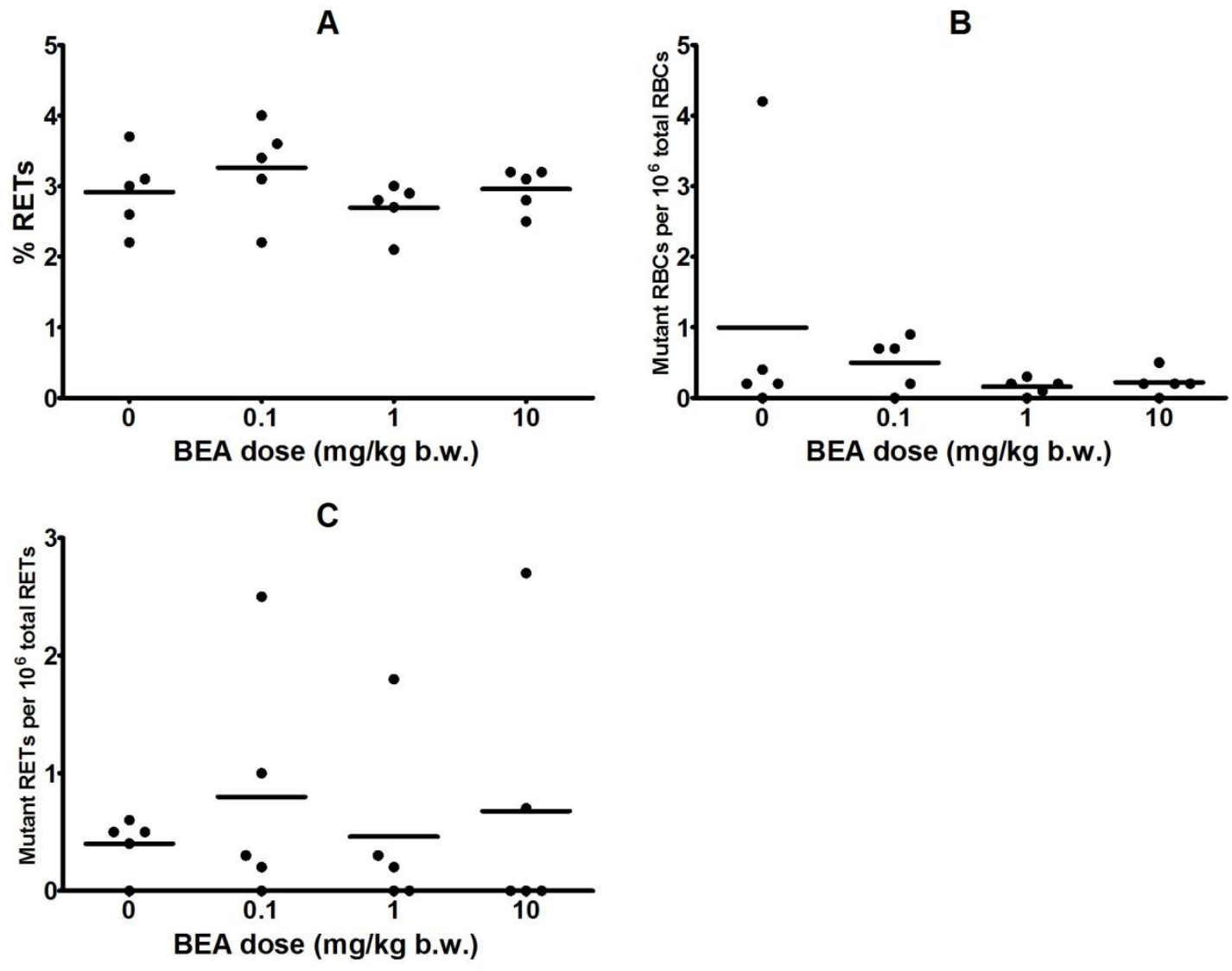

Figure 35: Pig-a assay in red blood cells of male mice after repeated oral treatments with different doses of BEA ( 0 - olive oil; $0.1,1$ and $10 \mathrm{mg} / \mathrm{kg}$ per day). Dots represent the percentage of RETs (A), the frequency of mutant RBCs (B) and the frequency of mutant RETs (C) obtained for each animal and lines represent the mean for each group.

\section{Micronucleus test}

The complete set of parameters obtained in male mice are reported in Table 23. In the same table also results of the negative and positive CTRL groups are reported. MMS treatment, used as a positive 
CTRL, induced a significant increase of micronucleated RETs and a decrease of the \% of reticulocytes. No increase of micronucleated cells and no induction of toxicity were observed after exposure to BEA as also shown in Figure 36. These results indicate that BEA was not clastogenic or aneugenic in bone marrow precursors of red blood cells and was not cytotoxic in the same cells.

Table 23: $\quad M N$ assays in male mice after repeated oral treatments with different doses of BEA ( 0 - olive oil; $0.1,1$ and $10 \mathrm{mg} / \mathrm{kg}$ per day).

\begin{tabular}{lcc}
\hline \multicolumn{1}{c}{ Treatment } & \%RETs & \%MN-RETs \\
\hline olive oil & $1.542 \pm 0.126$ & $0.126 \pm 0.025$ \\
BEA0.1 & $1.594 \pm 0.143$ & $0.144 \pm 0.027$ \\
BEA1 & $1.702 \pm 0.212$ & $0.158 \pm 0.026$ \\
BEA10 & $1.770 \pm 0.151$ & $0.186 \pm 0.031$ \\
MMS & $0.984 \pm 0.106 \#$ & $2.390 \pm 0.686 \#$ \\
\hline
\end{tabular}

$\# p<0.05$
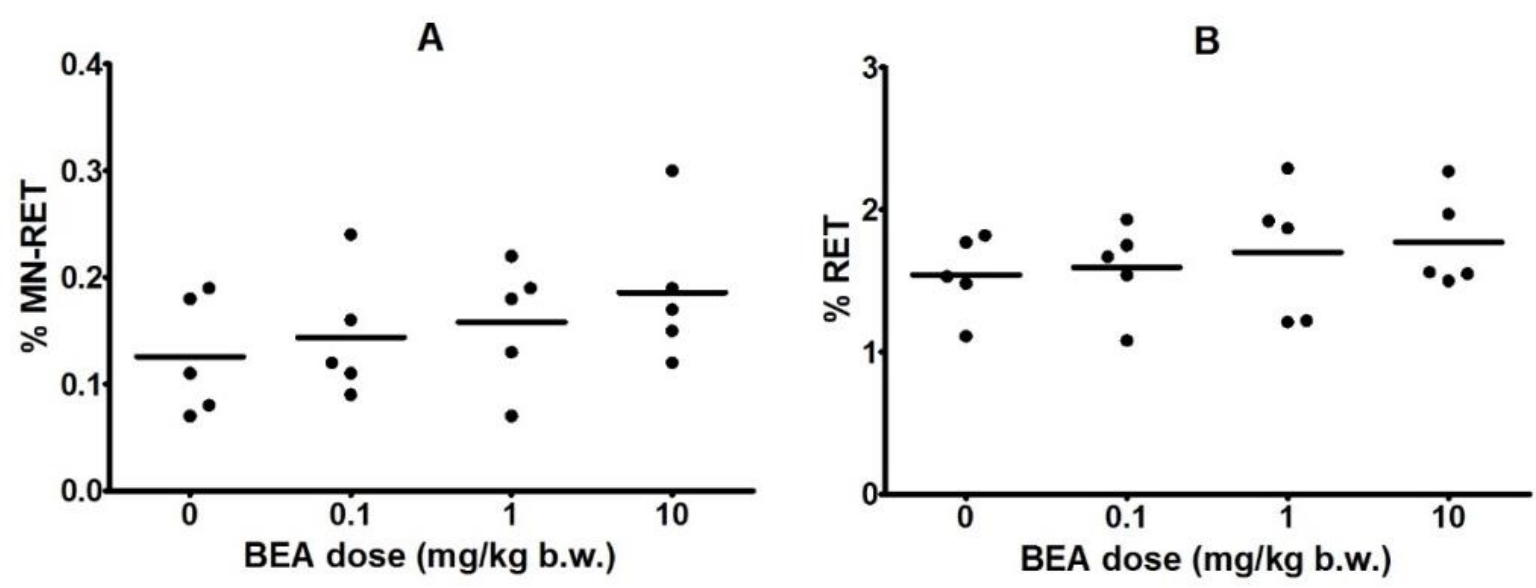

Figure 36: Micronucleus assay in reticulocytes of male mice after repeated oral treatments with different doses of BEA ( 0 - olive oil; $0.1,1$ and $10 \mathrm{mg} / \mathrm{kg}$ per day). Dots represent the percentage of micronucleated reticulocytes $(A)$ and percentage of RETs (B) obtained for each animal and lines represent the mean for each group. 


\subsection{6. $\quad$ ENNB - General toxicity}

\section{General toxicity}

A total of 6 mice died during the course of the study and were from the following groups/sex ( $M$ or $F$ ): ENNB0.18: 1 male and 1 female; ENNB18: 4 males. The cause of death was due to gavage procedure and the histopathological evaluation was not performed.

\section{Body weight}

The body weight of female mice at the end of the treatment period was significantly lower in ENNB0.18 and ENNB18 groups in comparison to CTRL (Fig. 37A), whereas the weight of male mice was significantly lower in the ENNB18 group.

\section{Food consumption}

The food consumption significantly increased in male mice of the ENNB18 group (Fig. 37B). No modification was recorded in female mice.
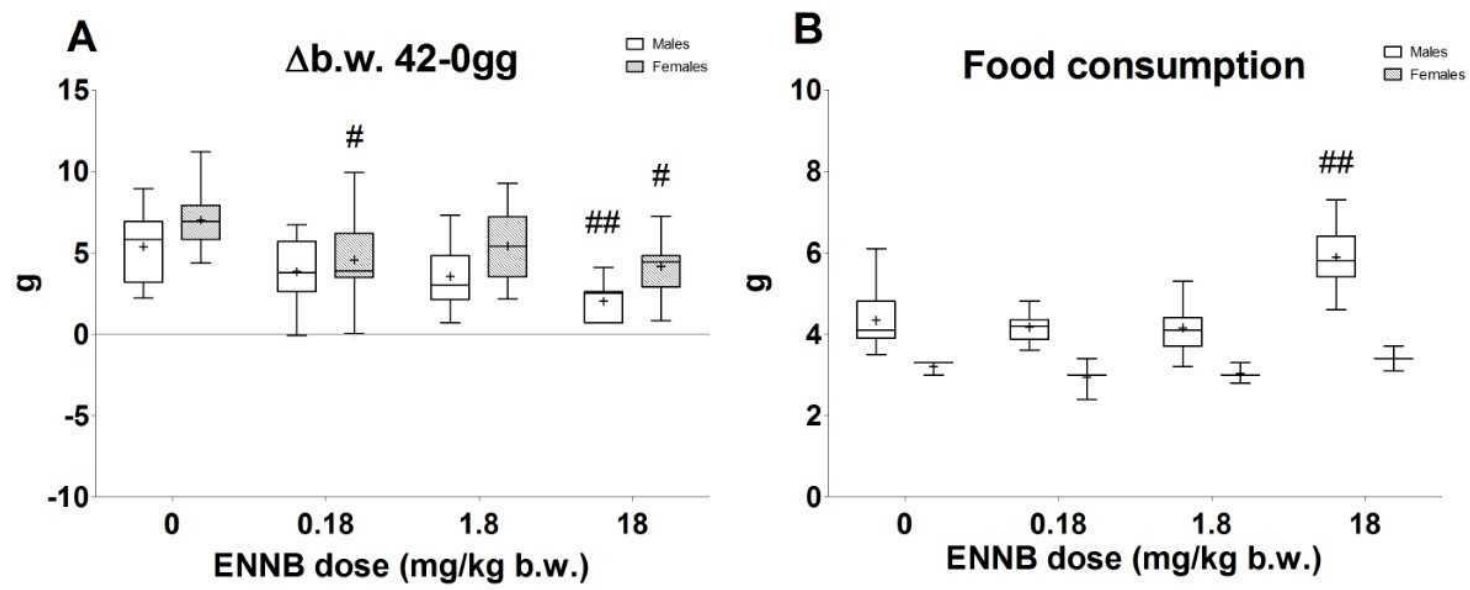

Figure 37: Mean difference in body weight (A) between the end (treatment day, TD42) and the beginning of the treatment (TD0) and mean food consumption (B) during the treatment period of male and female mice orally treated for 42 days with different doses of ENNB ( 0 - olive oil+DMSO6\%; $0.18,1.8$ and $18 \mathrm{mg} / \mathrm{kg}$ per day). Levels are reported as interquartile box plots, with whiskers delimiting the 10th and 90th percentiles. " + " indicates the mean value for each experimental group; $\# \mathrm{p}<0.05$.

\section{Organ weight}

Absolute and relative organ weights were summarized in Table 24.

The following organ weights were significantly changed in comparison to CTRL olive oil + DMSO6\%:

Liver absolute weight was decreased in female at ENNB0.18 and in males at ENNB18 (Fig. 38A).

Spleen relative weight was increased in males at ENNB18 (Fig. 38D).

Kidney absolute weight was decreased in females at ENNB1.8 and 18 (Fig. 38G).

Thymus absolute weight was decreased in females at ENNB0.18 and ENNB18, whereas relative weight was decreased only at ENNB0.18 (Fig. 38K-L).

Brain absolute weight was increased in males at ENNB0.18, relative weight was increased in males of all treatment groups and in females at ENNB18 (Fig. 38Q-R). 
Table 24: Absolute and relative organ weights of male and female mice orally treated for 42 days with different doses of ENNB ( 0 - olive oil+DMSO6\%; $0.18,1.8$ and $18 \mathrm{mg} / \mathrm{kg}$ per day).

\begin{tabular}{|c|c|c|c|c|c|c|c|c|c|}
\hline \multirow[b]{2}{*}{ ORGAN } & & \multicolumn{4}{|c|}{ Males } & \multicolumn{4}{|c|}{ Females } \\
\hline & & Olive oil+DMSO6\% & ENNB0.18 & ENNB1.8 & ENNB18 & Olive oil+DMSO6\% & ENNB0.18 & ENNB1.8 & ENNB18 \\
\hline LIVER & $\begin{array}{r}N \text { of mice examined } \\
\text { Absolute weight }(\mathrm{g}) \\
\text { Relative weight }(\mathrm{x} 100)\end{array}$ & $\begin{array}{c}\text { mean } \pm \text { SD } \\
11 \\
1.95 \pm 0.28 \\
4.81 \pm 0.54\end{array}$ & $\begin{array}{c}\text { mean } \pm S D \\
10 \\
1.71 \pm 0.23 \\
4.61 \pm 0.69\end{array}$ & $\begin{array}{c}\text { mean } \pm \text { SD } \\
11 \\
1.75 \pm 0.19 \\
4.86 \pm 0.55\end{array}$ & $\begin{array}{c}\text { mean } \pm \text { SD } \\
7 \\
1.63 \pm 0.16 \# \\
4.61 \pm 0.50\end{array}$ & $\begin{array}{c}\text { mean } \pm \text { SD } \\
11 \\
1.58 \pm 0.19 \\
4.82 \pm 0.72\end{array}$ & $\begin{array}{c}\text { mean } \pm \text { SD } \\
10 \\
1.32 \pm 0.27 \# \\
4.43 \pm 0.14\end{array}$ & $\begin{array}{c}\text { mean } \pm S D \\
11 \\
1.42 \pm 0.23 \\
4.62 \pm 0.54\end{array}$ & $\begin{array}{c}\text { mean } \pm \text { SD } \\
11 \\
1.38 \pm 0.24 \\
4.84 \pm 0.80\end{array}$ \\
\hline SPLEEN & $\begin{array}{r}N \text { of mice examined } \\
\text { Absolute weight }(\mathrm{g}) \\
\text { Relative weight }(x 100)\end{array}$ & $\begin{array}{c}11 \\
0.10 \pm 0.01 \\
0.25 \pm 0.02\end{array}$ & $\begin{array}{c}10 \\
0.10 \pm 0.02 \\
0.15 \pm 0.12\end{array}$ & $\begin{array}{c}11 \\
0.16 \pm 0.22 \\
0.43 \pm 0.58\end{array}$ & $\begin{array}{c}7 \\
0.16 \pm 0.07 \\
0.45 \pm 0.20 \#\end{array}$ & $\begin{array}{c}11 \\
0.16 \pm 0.03 \\
0.48 \pm 0.09\end{array}$ & $\begin{array}{c}10 \\
0.13 \pm 0.02 \\
0.44 \pm 0.07\end{array}$ & $\begin{array}{c}11 \\
0.14 \pm 0.02 \\
0.45 \pm 0.07\end{array}$ & $\begin{array}{c}11 \\
0.14 \pm 0.04 \\
0.49 \pm 0.14\end{array}$ \\
\hline MLN & $\begin{array}{r}N \text { of mice examined } \\
\text { Absolute weight }(\mathrm{g}) \\
\text { Relative weight }(\mathrm{x} 100)\end{array}$ & $\begin{array}{c}10 \\
0.03 \pm 0.01 \\
0.08 \pm 0.03\end{array}$ & $\begin{array}{c}10 \\
0.02 \pm 0.01 \\
0.06 \pm 0.03\end{array}$ & $\begin{array}{c}11 \\
0.03 \pm 0.01 \\
0.07 \pm 0.03\end{array}$ & $\begin{array}{c}7 \\
0.02 \pm 0.01 \\
0.06 \pm 0.04\end{array}$ & $\begin{array}{c}11 \\
0.02 \pm 0.01 \\
0.08 \pm 0.02\end{array}$ & $\begin{array}{c}9 \\
0.02 \pm 0.01 \\
0.06 \pm 0.03\end{array}$ & $\begin{array}{c}11 \\
0.04 \pm 0.05 \\
0.13 \pm 0.16\end{array}$ & $\begin{array}{c}10 \\
0.04 \pm 0.05 \\
0.14 \pm 0.17\end{array}$ \\
\hline KIDNEYS & $\begin{array}{r}N \text { of mice examined } \\
\text { Absolute weight }(\mathrm{g}) \\
\text { Relative weight }(\mathrm{x} 100)\end{array}$ & $\begin{array}{c}11 \\
0.55 \pm 0.05 \\
1.36 \pm 0.14\end{array}$ & $\begin{array}{c}10 \\
0.58 \pm 0.08 \\
1.55 \pm 0.17\end{array}$ & $\begin{array}{c}11 \\
0.55 \pm 0.07 \\
1.53 \pm 0.16\end{array}$ & $\begin{array}{c}7 \\
0.55 \pm 0.06 \\
1.57 \pm 0.26\end{array}$ & $\begin{array}{c}11 \\
0.42 \pm 0.07 \\
1.28 \pm 0.24\end{array}$ & $\begin{array}{c}10 \\
0.39 \pm 0.05 \\
1.31 \pm 0.25\end{array}$ & $\begin{array}{c}11 \\
0.36 \pm 0.05 \# \\
1.17 \pm 0.11\end{array}$ & $\begin{array}{c}11 \\
0.30 \pm 0.13 \# \\
1.06 \pm 0.48\end{array}$ \\
\hline ADRENALS & $\begin{array}{r}N \text { of mice examined } \\
\text { Absolute weight }(\mathrm{g}) \\
\text { Relative weight }(\mathrm{x} 100)\end{array}$ & $\begin{array}{c}11 \\
0.02 \pm 0.01 \\
0.05 \pm 0.02\end{array}$ & $\begin{array}{c}10 \\
0.02 \pm 0.01 \\
0.05 \pm 0.01\end{array}$ & $\begin{array}{c}11 \\
0.02 \pm 0.00 \\
0.05 \pm 0.01\end{array}$ & $\begin{array}{c}7 \\
0.02 \pm 0.01 \\
0.05 \pm 0.02\end{array}$ & $\begin{array}{c}9 \\
0.02 \pm 0.00 \\
0.06 \pm 0.01\end{array}$ & $\begin{array}{c}10 \\
0.02 \pm 0.01 \\
0.05 \pm 0.03\end{array}$ & $\begin{array}{c}10 \\
0.02 \pm 0.00 \\
0.06 \pm 0.01\end{array}$ & $\begin{array}{c}9 \\
0.03 \pm 0.07 \\
0.12 \pm 0.21\end{array}$ \\
\hline THYMUS & $\begin{array}{r}N \text { of mice examined } \\
\text { Absolute weight }(\mathrm{g}) \\
\text { Relative weight }(\mathrm{x} 100)\end{array}$ & $\begin{array}{c}11 \\
0.12 \pm 0.24 \\
0.33 \pm 0.69\end{array}$ & $\begin{array}{c}10 \\
0.05 \pm 0.02 \\
0.13 \pm 0.04\end{array}$ & $\begin{array}{c}11 \\
0.04 \pm 0.01 \\
0.12 \pm 0.04\end{array}$ & $\begin{array}{c}7 \\
0.05 \pm 0.03 \\
0.13 \pm 0.07\end{array}$ & $\begin{array}{c}11 \\
0.07 \pm 0.01 \\
0.21 \pm 0.04\end{array}$ & $\begin{array}{c}10 \\
0.05 \pm 0.01 \# \# \\
0.16 \pm 0.04 \#\end{array}$ & $\begin{array}{c}9 \\
0.06 \pm 0.01 \\
0.19 \pm 0.05\end{array}$ & $\begin{array}{c}10 \\
0.05 \pm 0.02 \# \\
0.19 \pm 0.05\end{array}$ \\
\hline HEART & $\begin{array}{r}N \text { of mice examined } \\
\text { Absolute weight }(\mathrm{g}) \\
\text { Relative weight }(x 100)\end{array}$ & $\begin{array}{c}11 \\
0.24 \pm 0.05 \\
0.58 \pm 0.10\end{array}$ & $\begin{array}{c}10 \\
0.21 \pm 0.02 \\
0.56 \pm 0.06\end{array}$ & $\begin{array}{c}11 \\
0.21 \pm 0.03 \\
0.57 \pm 0.07\end{array}$ & $\begin{array}{c}7 \\
0.22 \pm 0.02 \\
0.57 \pm 0.11\end{array}$ & $\begin{array}{c}11 \\
0.19 \pm 0.04 \\
0.58 \pm 0.13\end{array}$ & $\begin{array}{c}10 \\
0.19 \pm 0.03 \\
0.65 \pm 0.13\end{array}$ & $\begin{array}{c}11 \\
0.18 \pm 0.03 \\
0.57 \pm 0.07\end{array}$ & $\begin{array}{c}11 \\
0.16 \pm 0.03 \\
0.57 \pm 0.11\end{array}$ \\
\hline THYROID & $\begin{array}{r}N \text { of mice examined } \\
\text { Absolute weight }(\mathrm{g}) \\
\text { Relative weight }(\mathrm{x} 100)\end{array}$ & $\begin{array}{c}7 \\
0.006 \pm 0.001 \\
0.014 \pm 0.003\end{array}$ & $\begin{array}{c}7 \\
0.004 \pm 0.001 \\
0.011 \pm 0.004\end{array}$ & $\begin{array}{c}6 \\
0.006 \pm 0.002 \\
0.016 \pm 0.004\end{array}$ & $\begin{array}{c}7 \\
0.005 \pm 0.001 \\
0.013 \pm 0.003\end{array}$ & $\begin{array}{c}6 \\
0.006 \pm 0.001 \\
0.017 \pm 0.003\end{array}$ & $\begin{array}{c}7 \\
0.006 \pm 0.002 \\
0.019 \pm 0.007\end{array}$ & $\begin{array}{c}6 \\
0.006 \pm 0.002 \\
0.018 \pm 0.006\end{array}$ & $\begin{array}{c}6 \\
0.006 \pm 0.001 \\
0.020 \pm 0.004\end{array}$ \\
\hline BRAIN & $\begin{array}{r}N \text { of mice examined } \\
\text { Absolute weight }(\mathrm{g}) \\
\text { Relative weight }(\times 100)\end{array}$ & $\begin{array}{c}11 \\
0.41 \pm 0.04 \\
1.01 \pm 0.08\end{array}$ & $\begin{array}{c}10 \\
0.47 \pm 0.11 \# \\
1.27 \pm 0.32 \# \#\end{array}$ & $\begin{array}{c}11 \\
0.43 \pm 0.02 \\
1.18 \pm 0.07 \# \# \#\end{array}$ & $\begin{array}{c}7 \\
0.41 \pm 0.04 \\
1.14 \pm 0.08 \#\end{array}$ & $\begin{array}{c}11 \\
0.43 \pm 0.03 \\
1.29 \pm 0.11\end{array}$ & $\begin{array}{c}10 \\
0.38 \pm 0.06 \\
1.27 \pm 0.22\end{array}$ & $\begin{array}{c}11 \\
0.42 \pm 0.07 \\
1.36 \pm 0.12\end{array}$ & $\begin{array}{c}11 \\
0.43 \pm 0.04 \\
1.52 \pm 0.21 \# \#\end{array}$ \\
\hline
\end{tabular}


Table 24 (continued): Absolute and relative organ weights of male and female mice orally treated for 42 days with different doses of ENNB ( 0 - olive oil+DMSO6\%; 0.18, 1.8 and $18 \mathrm{mg} / \mathrm{kg}$ per day).

\begin{tabular}{|c|c|c|c|c|c|c|c|c|c|}
\hline \multirow[b]{2}{*}{ ORGAN } & & \multicolumn{4}{|c|}{ Males } & \multicolumn{4}{|c|}{ Females } \\
\hline & & Olive oil+DMSO6\% & ENNB0.18 & ENNB1.8 & ENNB18 & Olive oil+DMSO6\% & ENNB0.18 & ENNB1.8 & ENNB18 \\
\hline OVARIES & $\begin{array}{r}N \text { of mice examined } \\
\text { Absolute weight }(\mathrm{g}) \\
\text { Relative weight }\end{array}$ & & & & & $\begin{array}{c}11 \\
0.07 \pm 0.02 \\
0.20 \pm 0.06\end{array}$ & $\begin{array}{c}10 \\
0.09 \pm 0.02 \\
0.28 \pm 0.07\end{array}$ & $\begin{array}{c}11 \\
0.09 \pm 0.03 \\
0.29 \pm 0.08\end{array}$ & $\begin{array}{c}11 \\
0.04 \pm 0.01 \\
0.16 \pm 0.05\end{array}$ \\
\hline UTERUS & $\begin{array}{r}N \text { of mice examined } \\
\text { Absolute weight }(\mathrm{g}) \\
\text { Relative weight }(\mathrm{x} 100) \\
\end{array}$ & & & & & $\begin{array}{c}11 \\
0.24 \pm 0.06 \\
0.72 \pm 0.17\end{array}$ & $\begin{array}{c}10 \\
0.27 \pm 0.10 \\
0.89 \pm 0.34\end{array}$ & $\begin{array}{c}11 \\
0.20 \pm 0.08 \\
0.63 \pm 0.23\end{array}$ & $\begin{array}{c}11 \\
0.19 \pm 0.09 \\
0.67 \pm 0.32\end{array}$ \\
\hline TESTIES & $\begin{array}{r}N \text { of mice examined } \\
\text { Absolute weight }(\mathrm{g}) \\
\text { Relative weight }(\mathrm{x} 100)\end{array}$ & $\begin{array}{c}11 \\
0.28 \pm 0.03 \\
0.70 \pm 0.03\end{array}$ & $\begin{array}{c}10 \\
0.26 \pm 0.04 \\
0.70 \pm 0.09\end{array}$ & $\begin{array}{c}11 \\
0.26 \pm 0.02 \\
0.73 \pm 0.08\end{array}$ & $\begin{array}{c}7 \\
0.28 \pm 0.03 \\
0.78 \pm 0.09\end{array}$ & & & & \\
\hline EPIDIDIMUS & $\begin{array}{r}N \text { of mice examined } \\
\text { Absolute weight }(\mathrm{g}) \\
\text { Relative weight }(x 100)\end{array}$ & $\begin{array}{c}11 \\
0.19 \pm 0.05 \\
0.48 \pm 0.12\end{array}$ & $\begin{array}{c}10 \\
0.18 \pm 0.02 \\
0.47 \pm 0.06\end{array}$ & $\begin{array}{c}11 \\
0.16 \pm 0.03 \\
0.45 \pm 0.07\end{array}$ & $\begin{array}{c}7 \\
0.19 \pm 0.03 \\
0.54 \pm 0.15\end{array}$ & & & & \\
\hline
\end{tabular}



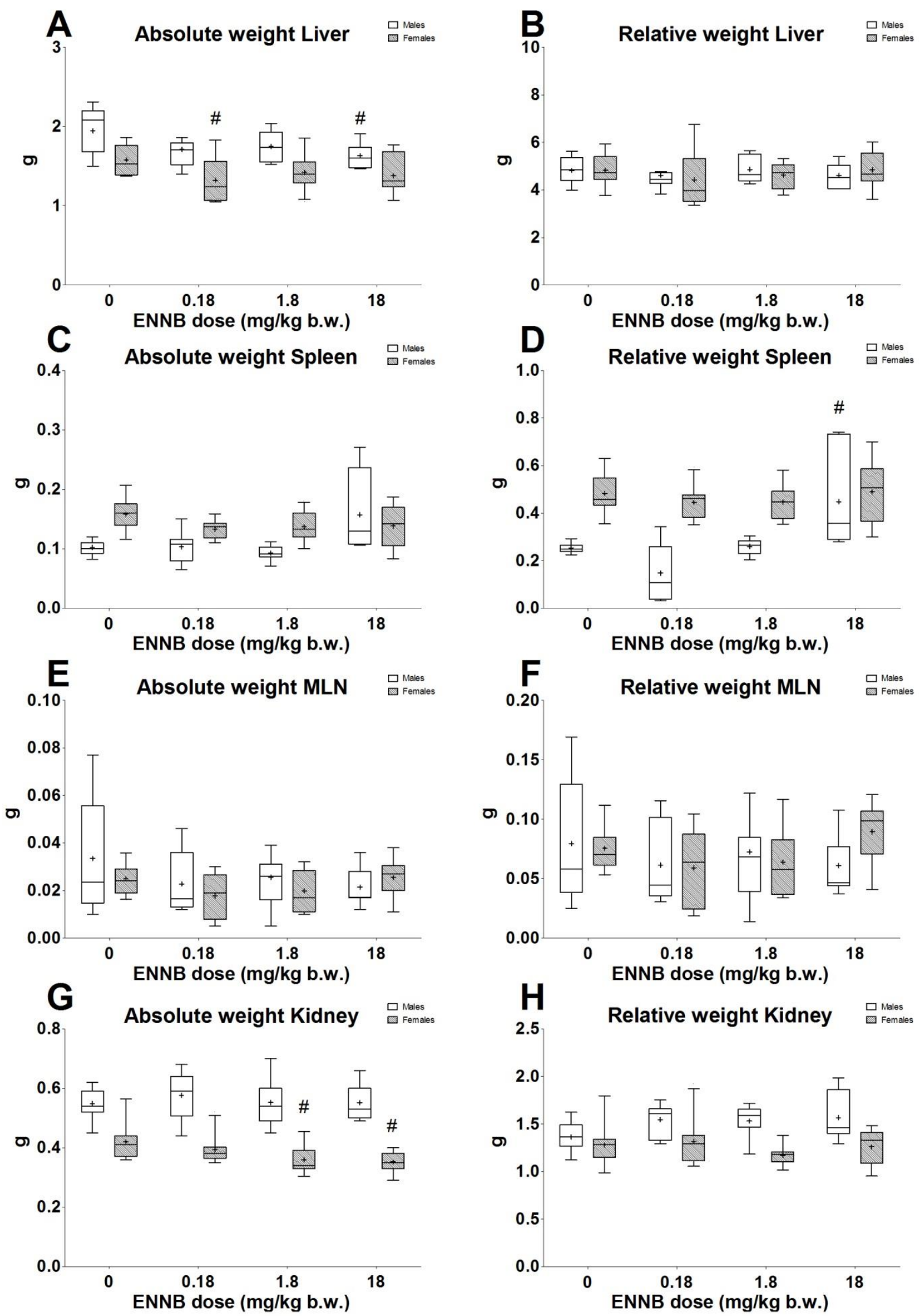

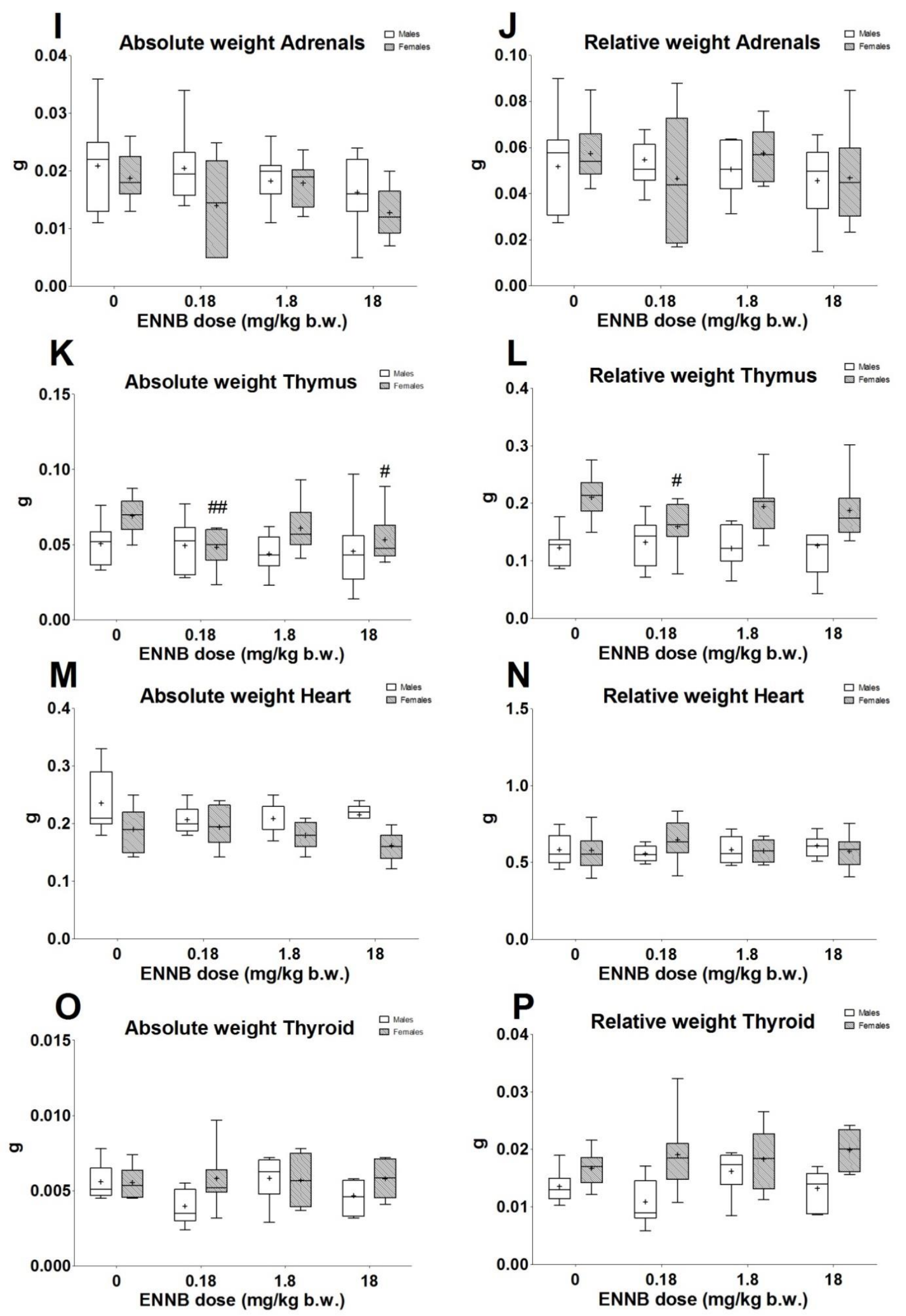

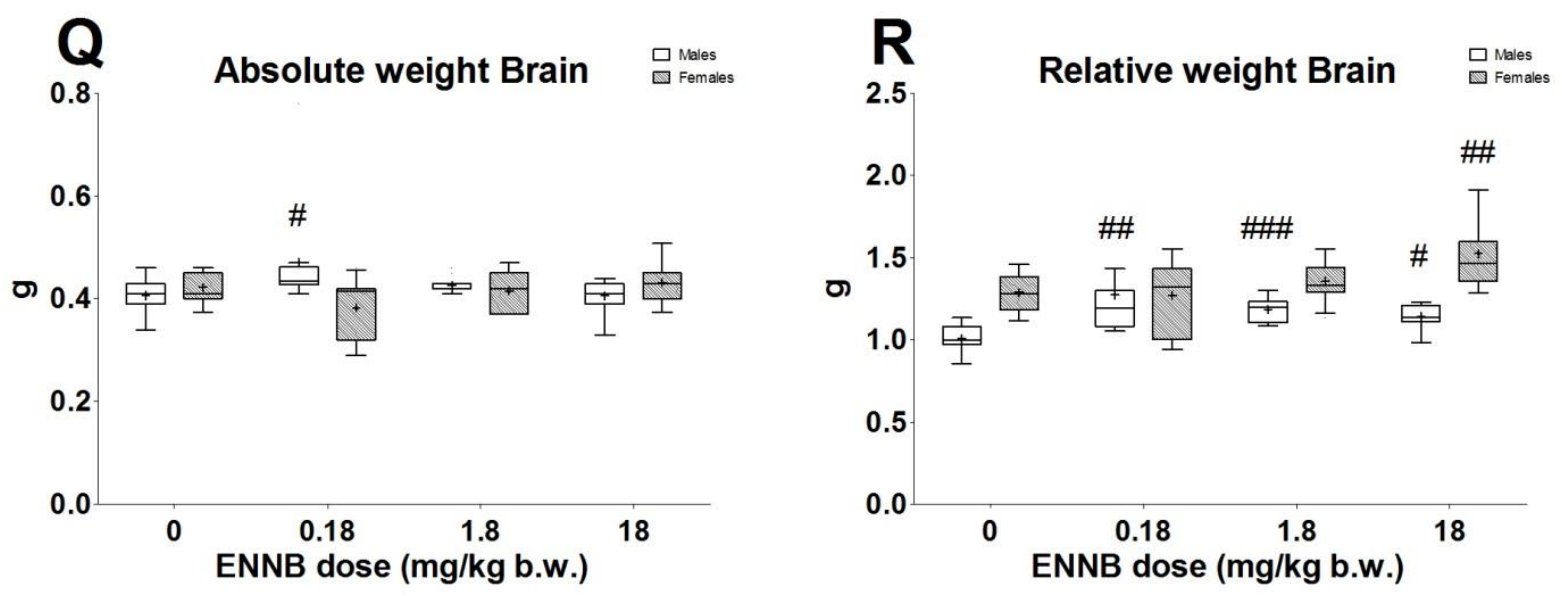

Figure 38: Absolute and relative organ weights of male (white box) and female (striped box) mice orally treated for 42 days with different doses of ENNB ( 0 - olive oil+DMSO6\%; 0.18 , 1.8 and $18 \mathrm{mg} / \mathrm{kg}$ per day). Levels are reported as interquartile box plots, with whiskers delimiting the 10th and 90th percentiles. "+" indicates the mean value for each experimental group; \# $\mathrm{p}<0.05$; \# $\mathrm{p}<0.01$; \#\# $\mathrm{p}<0.001$.

No significant effect was observed on reproductive organ weight in both sexes (Fig. 39). 

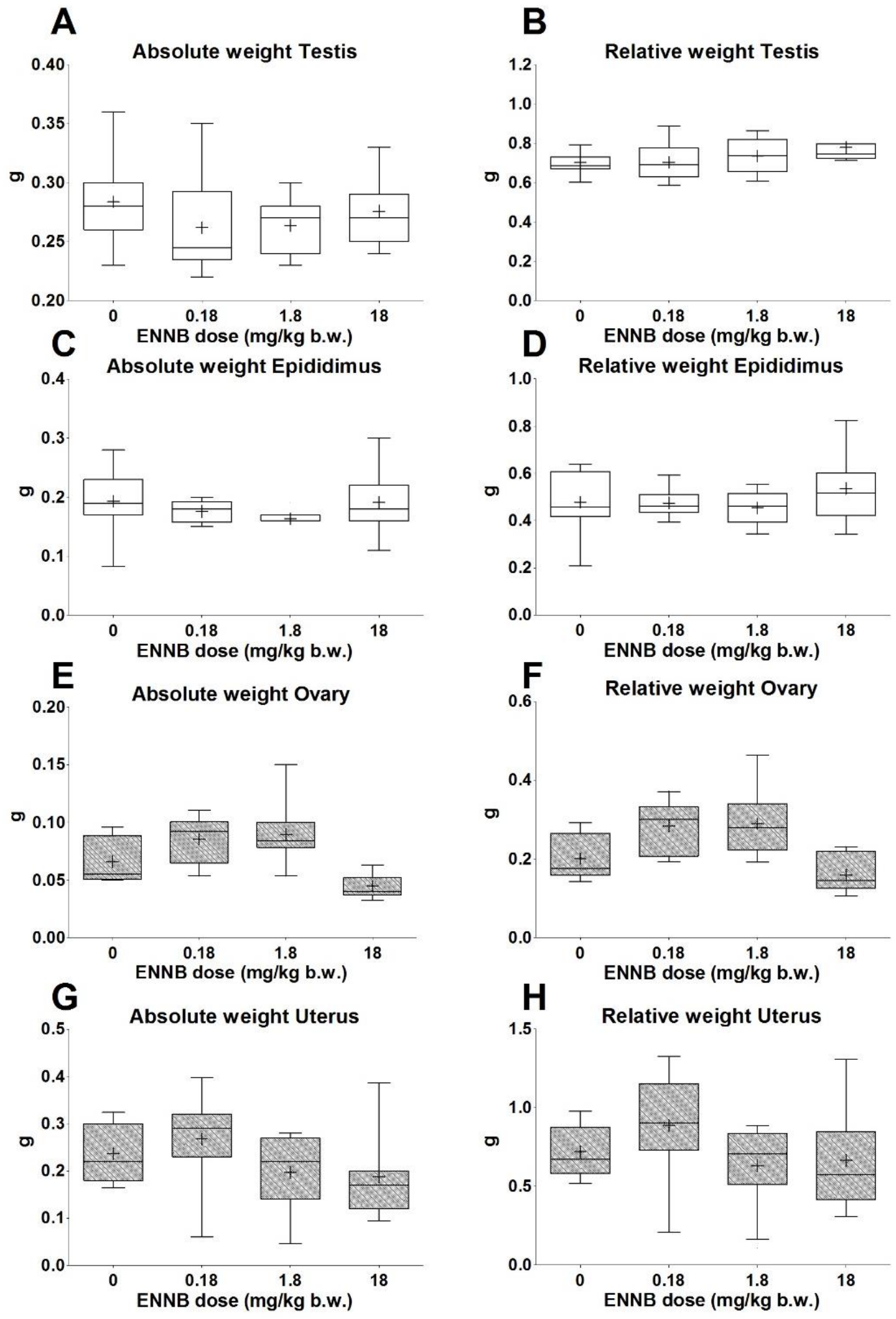

Figure 39: Absolute and relative weight of reproductive organs of male (white box) and female (striped box) mice orally treated for 42 days with different doses of ENNB ( 0 - olive oil+DMSO6\%; $0.18,1.8$ and $18 \mathrm{mg} / \mathrm{kg}$ per day). Levels are reported as interquartile box plots, with whiskers delimiting the 10th and 90th percentiles. " + " indicates the mean value for each experimental group. 


\subsubsection{ENNB - Histopathological endpoints}

The histopathological data are summarized in Table 25.

No histopathological alterations were present in mammary gland for all mice analyzed (data not shown). 
Table 25: Incidence of each histopathological endpoint analyzed in male and female mice orally treated for 42 days with different doses of ENNB (0 olive oil+DMSO6\%; 0.18, 1.8 and $18 \mathrm{mg} / \mathrm{kg}$ per day).

\begin{tabular}{|c|c|c|c|c|c|c|c|c|c|}
\hline \multirow[b]{2}{*}{ ORGAN/Effect } & & \multicolumn{4}{|c|}{ Males } & \multicolumn{4}{|c|}{ Females } \\
\hline & & $\begin{array}{c}\text { Olive } \\
\text { oil+DMSO } \\
6 \% \\
\end{array}$ & ENNB0.18 & ENNB1.8 & ENNB18 & $\begin{array}{c}\text { Olive } \\
\text { oil+DMSO } \\
6 \% \\
\end{array}$ & ENNB0.18 & ENNB1.8 & ENNB18 \\
\hline \multirow[t]{6}{*}{$\begin{array}{l}\text { THYROID } \\
\text { Colloid foaminess }\end{array}$} & $N$ of mice examined & 6 & 6 & 6 & 6 & 6 & 7 & 5 & 6 \\
\hline & 0 & 4 & 2 & 2 & 2 & 3 & 2 & 1 & 5 \\
\hline & 1 & 1 & 4 & 3 & 1 & 3 & 3 & 4 & 1 \\
\hline & 2 & 1 & & 1 & 2 & & 2 & & \\
\hline & 3 & & & & 1 & & & & \\
\hline & Total Finding Incidence & 2 & 4 & 4 & 4 & 3 & 5 & 4 & 1 \\
\hline \multicolumn{2}{|c|}{ Necrotic cells exfoliated into the colloid } & & & & & & & & \\
\hline & (2) & 5 & 6 & 5 & 6 & 6 & 6 & 5 & 4 \\
\hline & 1 & 1 & & & & & 1 & & 2 \\
\hline & 2 & & & 1 & & & & & \\
\hline & Total Finding Incidence & 1 & 0 & 1 & 0 & 0 & 1 & 0 & 2 \\
\hline \multicolumn{2}{|c|}{ Colloid reduction/absence in follicles } & & & & & & & & \\
\hline & 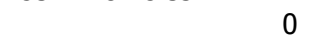 & 6 & 6 & 6 & 6 & 6 & 6 & 5 & 3 \\
\hline & 1 & & & & & & 1 & & 3 \\
\hline & Total Finding Incidence & 0 & 0 & 0 & 0 & 0 & 1 & 0 & 3 \\
\hline \multirow[t]{4}{*}{ Pyknotic nuclei } & & & & & & & & & \\
\hline & 0 & 6 & 5 & 6 & 6 & 6 & 7 & 5 & 6 \\
\hline & 1 & & 1 & & & & & & \\
\hline & Total Finding Incidence & 0 & 1 & 0 & 0 & 0 & 0 & 0 & 0 \\
\hline \multirow[t]{4}{*}{ Follicles degenerati } & & & & & & & & & \\
\hline & $\begin{array}{l}0 \\
1\end{array}$ & 6 & 6 & 5 & 6 & 6 & 7 & 5 & $\begin{array}{l}5 \\
1\end{array}$ \\
\hline & 3 & & & 1 & & & & & \\
\hline & Total Finding Incidence & 0 & 0 & 1 & 0 & 0 & 0 & 0 & 1 \\
\hline
\end{tabular}

Grade 0: No change; Grade 1: Minimal; Grade 2: Mild; Grade 3: Moderate; Grade 4: Marked. 
Table 25 (continued): Incidence of each histopathological endpoint analyzed in male and female mice orally treated for 42 days with different doses of ENNB ( 0 - olive oil+DMSO6\%; $0.18,1.8$ and $18 \mathrm{mg} / \mathrm{kg}$ per day).

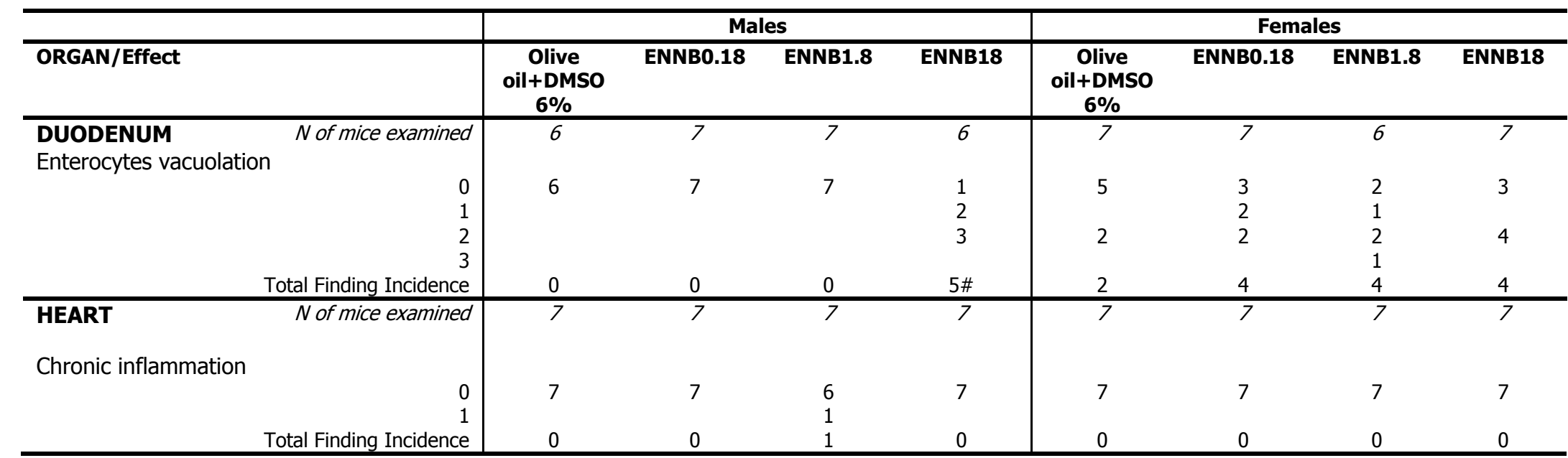

Grade 0: No change; Grade 1: Minimal; Grade 2: Mild; Grade 3: Moderate; Grade 4: Marked. \#p < 0.05 . 
Table 25(continued): Incidence of each histopathological endpoint analyzed in male and female mice orally treated for 42 days with different doses of ENNB ( 0 - olive oil+DMSO6\%; 0.18, 1.8 and $18 \mathrm{mg} / \mathrm{kg}$ per day).

\begin{tabular}{|c|c|c|c|c|c|c|c|c|}
\hline \multirow[b]{2}{*}{ ORGAN/Effect } & \multicolumn{4}{|c|}{ Males } & \multicolumn{4}{|c|}{ Females } \\
\hline & $\begin{array}{c}\text { Olive } \\
\text { oil+DMSO } \\
6 \% \\
\end{array}$ & ENNB0.18 & ENNB1.8 & ENNB18 & $\begin{array}{c}\text { Olive } \\
\text { oil+DMSO } \\
6 \% \\
\end{array}$ & ENNB0.18 & ENNB1.8 & ENNB18 \\
\hline N of mice examined & 7 & 7 & 7 & 7 & 7 & 7 & 7 & 7 \\
\hline $\begin{array}{r}0 \\
1 \\
2 \\
\text { Total Finding Incidence } \\
\end{array}$ & $\begin{array}{l}3 \\
2 \\
2 \\
4\end{array}$ & $\begin{array}{l}5 \\
1 \\
1 \\
2\end{array}$ & $\begin{array}{l}5 \\
1 \\
1 \\
2\end{array}$ & $\begin{array}{l}6 \\
1 \\
1\end{array}$ & $\begin{array}{l}3 \\
4 \\
4\end{array}$ & $\begin{array}{l}6 \\
1 \\
1\end{array}$ & $\begin{array}{l}2 \\
4 \\
1 \\
5\end{array}$ & $\begin{array}{l}4 \\
1 \\
2 \\
3\end{array}$ \\
\hline $\begin{array}{r}\text { Hepatocytic hypertrophy } \\
\quad \begin{array}{r}2 \\
\text { Total Finding Incidence }\end{array}\end{array}$ & $\begin{array}{l}7 \\
0 \\
\end{array}$ & $\begin{array}{l}7 \\
0\end{array}$ & $\begin{array}{l}5 \\
2 \\
2\end{array}$ & $\begin{array}{l}7 \\
0\end{array}$ & $\begin{array}{l}7 \\
0\end{array}$ & $\begin{array}{l}7 \\
0\end{array}$ & $\begin{array}{l}7 \\
0\end{array}$ & $\begin{array}{l}7 \\
0\end{array}$ \\
\hline $\begin{array}{l}\text { Hepatocytic vacuolation } \\
\\
\\
\text { Total Finding Incidence } \\
2 \\
\end{array}$ & $\begin{array}{l}6 \\
1 \\
1\end{array}$ & $\begin{array}{l}7 \\
0\end{array}$ & $\begin{array}{l}7 \\
0 \\
\end{array}$ & $\begin{array}{l}7 \\
0 \\
\end{array}$ & $\begin{array}{l}7 \\
0 \\
\end{array}$ & $\begin{array}{l}7 \\
0\end{array}$ & $\begin{array}{l}5 \\
2 \\
2\end{array}$ & $\begin{array}{l}6 \\
1 \\
1\end{array}$ \\
\hline $\begin{array}{rr}\text { Inflammatory cell foci } & 0 \\
& 1 \\
& \text { Total Finding Incidence }\end{array}$ & $\begin{array}{l}6 \\
1 \\
1\end{array}$ & $\begin{array}{l}6 \\
1 \\
1\end{array}$ & $\begin{array}{l}6 \\
1 \\
1\end{array}$ & $\begin{array}{l}6 \\
1 \\
1\end{array}$ & $\begin{array}{l}6 \\
1 \\
1\end{array}$ & $\begin{array}{l}3 \\
4 \\
4\end{array}$ & $\begin{array}{l}7 \\
0\end{array}$ & $\begin{array}{l}6 \\
1 \\
1\end{array}$ \\
\hline $\begin{array}{lr}\text { SPLEEN } & N \text { of mice examined } \\
\text { Extramedullary haematopoiesis } & 0 \\
& 1 \\
& 3 \\
& \text { Total Finding Incidence }\end{array}$ & $\begin{array}{l}4 \\
2 \\
6\end{array}$ & $\begin{array}{l}3 \\
1 \\
1 \\
5\end{array}$ & $\begin{array}{l}5 \\
1 \\
6\end{array}$ & $\begin{array}{l}1 \\
2 \\
2 \\
5\end{array}$ & $\begin{array}{l}1 \\
4 \\
5\end{array}$ & $\begin{array}{l}3 \\
1 \\
4\end{array}$ & $\begin{array}{c}6 \\
1 \\
5 \\
5 \#\end{array}$ & $\begin{array}{l}5 \\
\\
1 \\
2 \\
2 \\
5\end{array}$ \\
\hline
\end{tabular}

Grade 0: No change; Grade 1: Minimal; Grade 2: Mild; Grade 3: Moderate; Grade 4: Marked. \#p < 0.05. 
Table 25 (continued): Incidence of each histopathological endpoint analyzed in male and female mice orally treated for 42 days with different doses of ENNB ( 0 - olive oil+DMSO6\%; 0.18, 1.8 and $18 \mathrm{mg} / \mathrm{kg}$ per day).

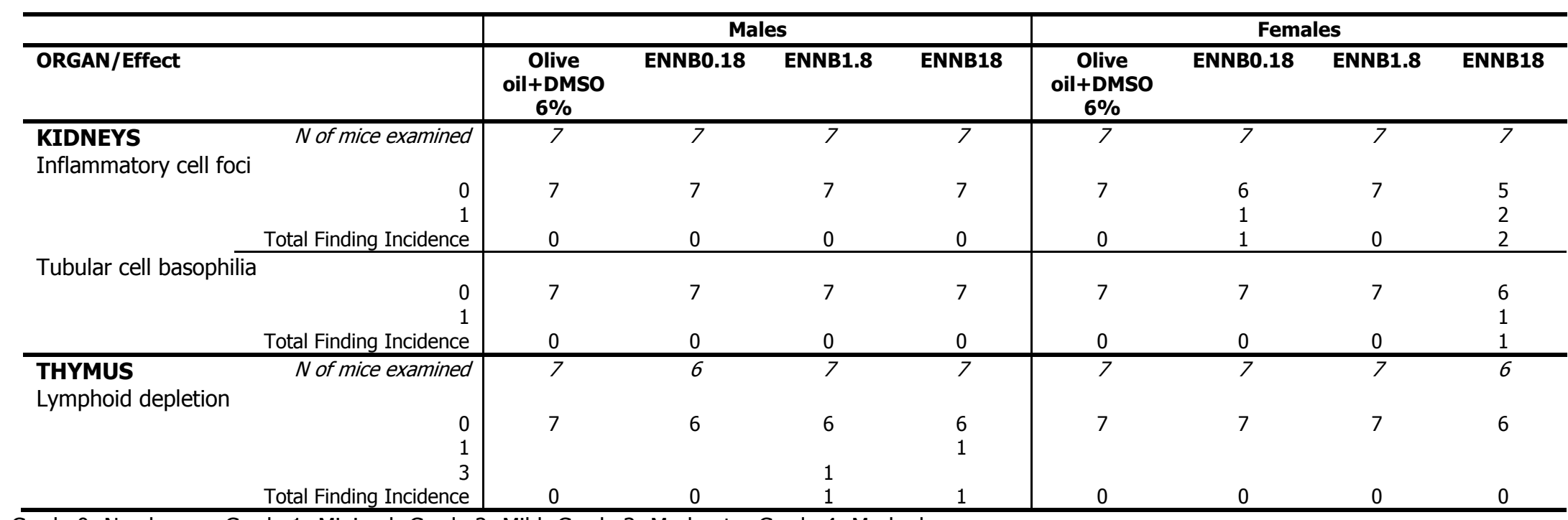


Table 25 (continued): Incidence of each histopathological endpoint analyzed in male and female mice orally treated for 42 days with different doses of ENNB ( 0 - olive oil+DMSO6\%; 0.18, 1.8 and $18 \mathrm{mg} / \mathrm{kg}$ per day).

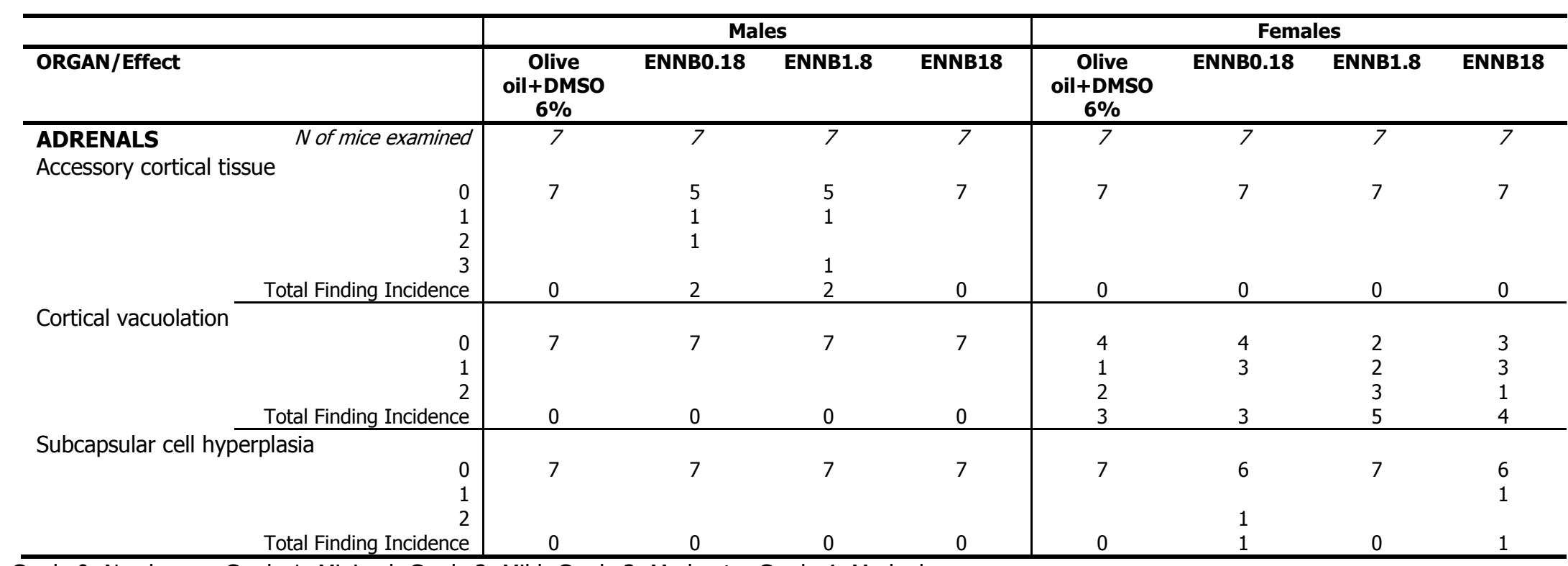


Table 25 (continued): Incidence of each histopathological endpoint analyzed in male and female mice orally treated for 42 days with different doses of ENNB ( 0 - olive oil+DMSO6\%; $0.18,1.8$ and $18 \mathrm{mg} / \mathrm{kg}$ per day).

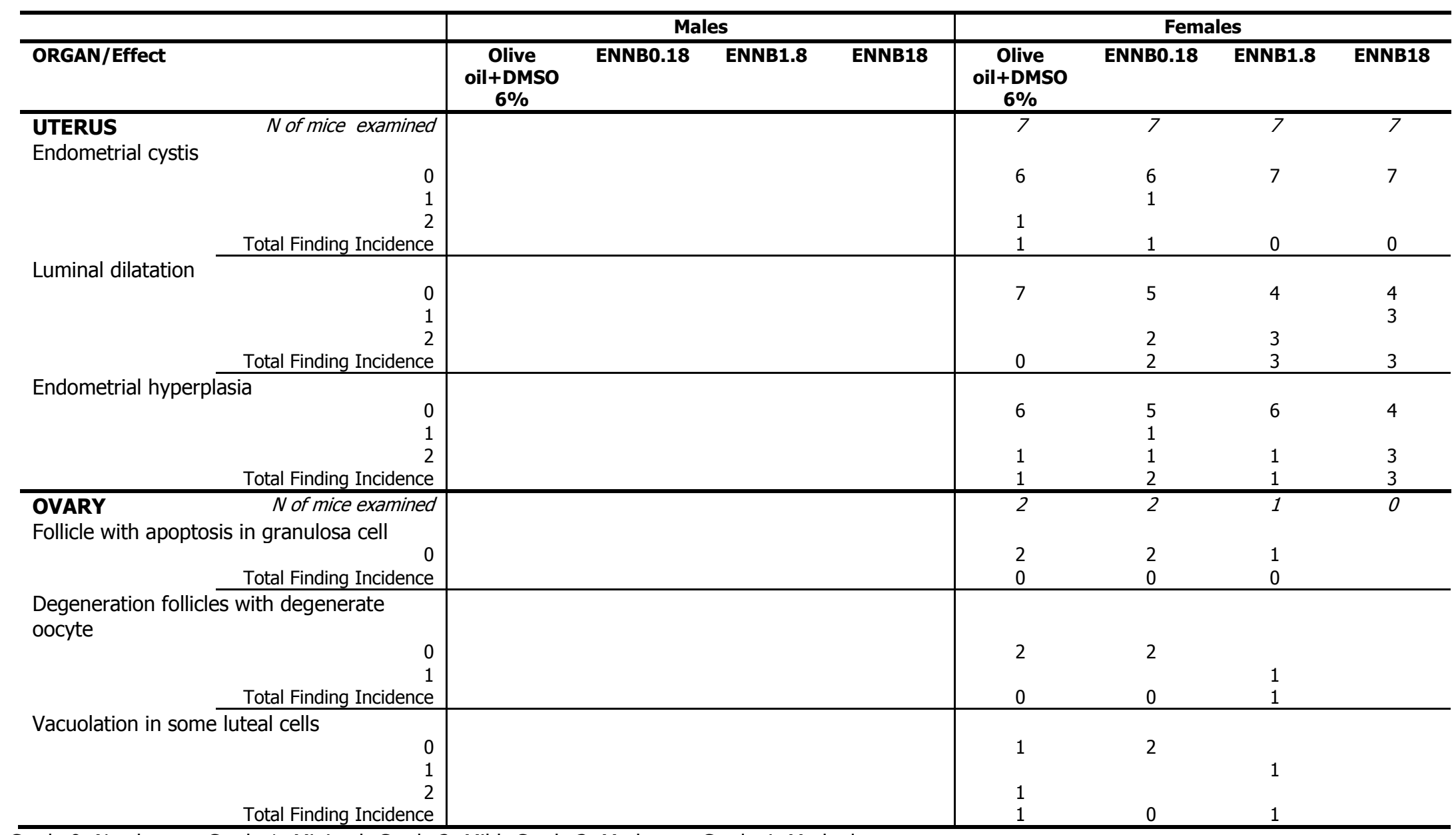

Grade 0: No change; Grade 1: Minimal; Grade 2: Mild; Grade 3: Moderate; Grade 4: Marked 
Table 25 (continued): Incidence of each histopathological endpoint analyzed in male and female mice orally treated for 42 days with different doses of ENNB (0 - olive oil+DMSO6\%; 0.18, 1.8 and $18 \mathrm{mg} / \mathrm{kg}$ per day).

\begin{tabular}{|c|c|c|c|c|c|c|c|c|}
\hline \multirow[b]{2}{*}{ ORGAN/Effect } & \multicolumn{4}{|c|}{ Males } & \multicolumn{4}{|c|}{ Females } \\
\hline & $\begin{array}{c}\text { Olive } \\
\text { oil+DMSO } \\
6 \% \\
\end{array}$ & ENNB0.18 & ENNB1.8 & ENNB18 & $\begin{array}{c}\text { Olive } \\
\text { oil+DMSO } \\
6 \% \\
\end{array}$ & ENNB0.18 & ENNB1.8 & ENNB18 \\
\hline N of mice examined & 7 & 7 & 7 & 7 & & & & \\
\hline $\begin{array}{r}0 \\
1\end{array}$ & $\begin{array}{l}5 \\
2\end{array}$ & 7 & $\begin{array}{l}5 \\
2\end{array}$ & 7 & & & & \\
\hline \begin{tabular}{cc} 
Total Finding Incidence \\
\cline { 2 - 3 }
\end{tabular} & 2 & 0 & 2 & 0 & & & & \\
\hline \multicolumn{9}{|l|}{ Caput: epithelial vacuolation } \\
\hline 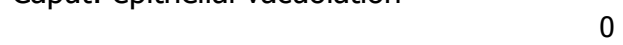 & 7 & 6 & 6 & 7 & & & & \\
\hline 1 & & 1 & 1 & & & & & \\
\hline \begin{tabular}{cc} 
Total Finding Incidence \\
\cline { 2 - 2 }
\end{tabular} & 0 & 1 & 1 & 0 & & & & \\
\hline \multicolumn{9}{|l|}{ Tubules degeneration } \\
\hline 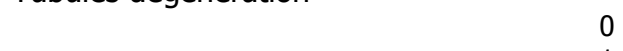 & 7 & 7 & 6 & 7 & & & & \\
\hline 1 & & & 1 & & & & & \\
\hline Total Finding Incidence & 0 & 0 & 1 & 0 & & & & \\
\hline$N$ of mice examined & 6 & 7 & 7 & 7 & & & & \\
\hline & mean \pm SE & mean $\pm S E$ & mean $\pm S E$ & mean $\pm S E$ & & & & \\
\hline$\%$ Unaffected tubules & $95.8 \pm 4.9$ & $97.9 \pm 3.9$ & $88.7 \pm 11.6$ & $87.1 \pm 16.0$ & & & & \\
\hline$\%$ Tubules with germ cell disorganization & $0.0 \pm 0.0$ & $0.0 \pm 0.0$ & $0.0 \pm 0.0$ & $2.9 \pm 4.9$ & & & & \\
\hline$\%$ Atrophic tubules & $0.8 \pm 2.0$ & $0.0 \pm 0.0$ & $0.0 \pm 0.0$ & $0.7 \pm 1.9$ & & & & \\
\hline$\%$ Tubules with vacuoles & $3.0 \pm 3.5$ & $0.7 \pm 1.9$ & $7.1 \pm 9.5$ & $4.3 \pm 5.4$ & & & & \\
\hline
\end{tabular}

Grade 0: No change; Grade 1: Minimal; Grade 2: Mild; Grade 3: Moderate; Grade 4: Marked.

*Testis: 50 different tubules/mice were observed and the scoring of lesions was done quantitatively, using percentages. 
Table 25 (continued): Incidence of each histopathological endpoint analyzed in male and female mice orally treated for 42 days with different doses of ENNB ( 0 - olive oil+DMSO6\%; $0.18,1.8$ and $18 \mathrm{mg} / \mathrm{kg}$ per day).

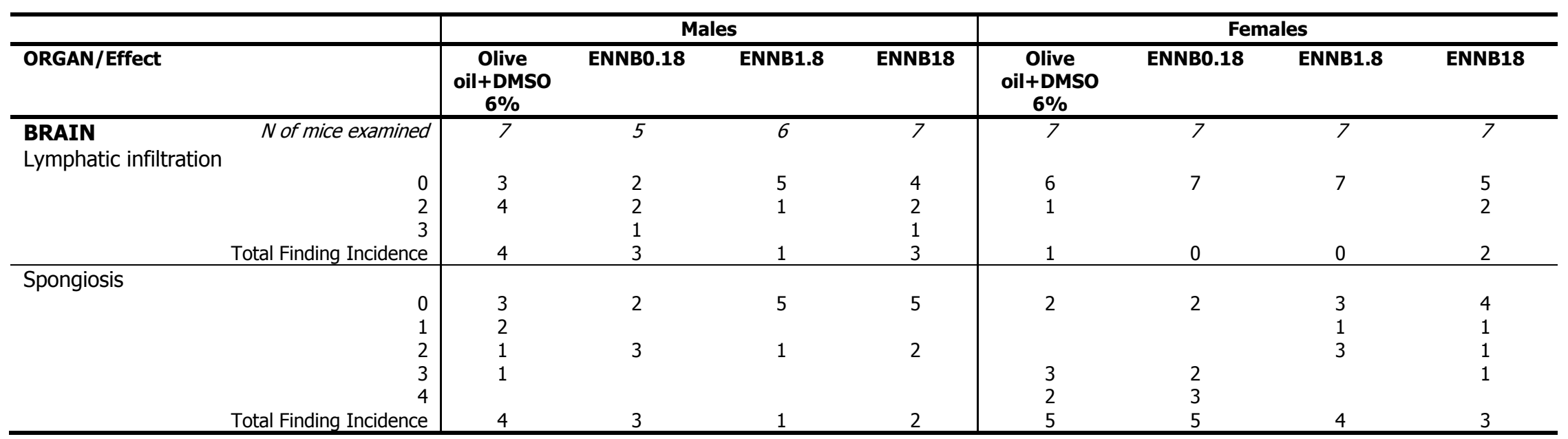

Grade 0: No change; Grade 1: Minimal; Grade 2: Mild; Grade 3: Moderate; Grade 4: Marked. 
No histopathological alterations were observed on thyroid in both sexes (Table 25). The only thyroid endpoint affected by ENNB treatment was the histomorphometrical measurement of number of thyroid follicles which was significantly increased by ENNB18 in male mice (Fig. 40A).

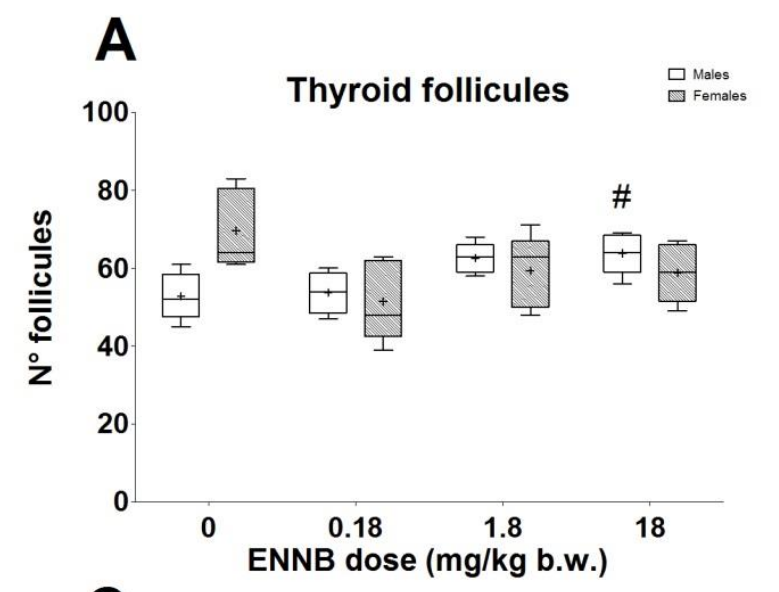

\section{B}
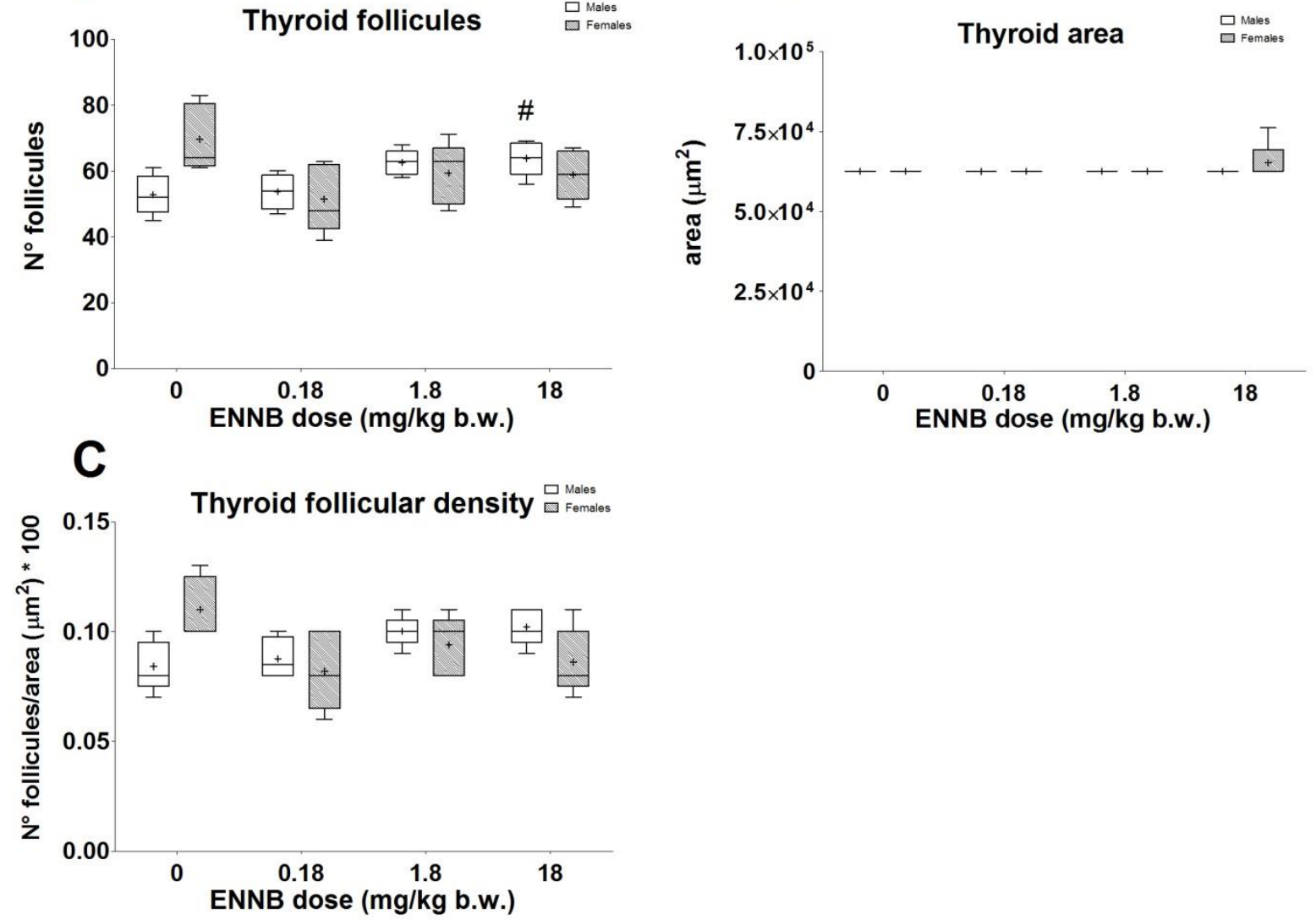

Figure 40: Histomorphometrical analysis of number of follicles (A), thyroid area (B), follicular density (C) in male (white box) and female (striped box) mice orally treated for 42 days with different doses of ENNB (0 - olive oil+DMSO6\%; 0.18, 1.8 and $18 \mathrm{mg} / \mathrm{kg}$ per day). Levels are reported as interquartile box plots, with whiskers delimiting the 10th and 90th percentiles. "+" indicates the mean value for each experimental group; \# $p<0.05$.

In the duodenum, enterocyte vacuolization was noted in high dose males, when compared with CTRL+DMSO6\% (Table 25). No effect was recorded in duodenum of female mice (Table 25).

No histopathological effects on heart and liver were observed in male and female mice (Table 25).

In spleen, no histopathological effect was observed in male, in female increased severity of extramedullary haematopoiesis (EMH) at BEA1.8 only (Table 25). Histomorphometrical analysis showed a significant increase in red and white pulp of female mice in the ENNB18 group; moreover white pulp was increased also by ENNB1.8 (Fig. 41A-B), the correlation with the treatment is unclear. The red/white spleen pulp ratio was not affected by ENNB treatment (Fig. 41C). 

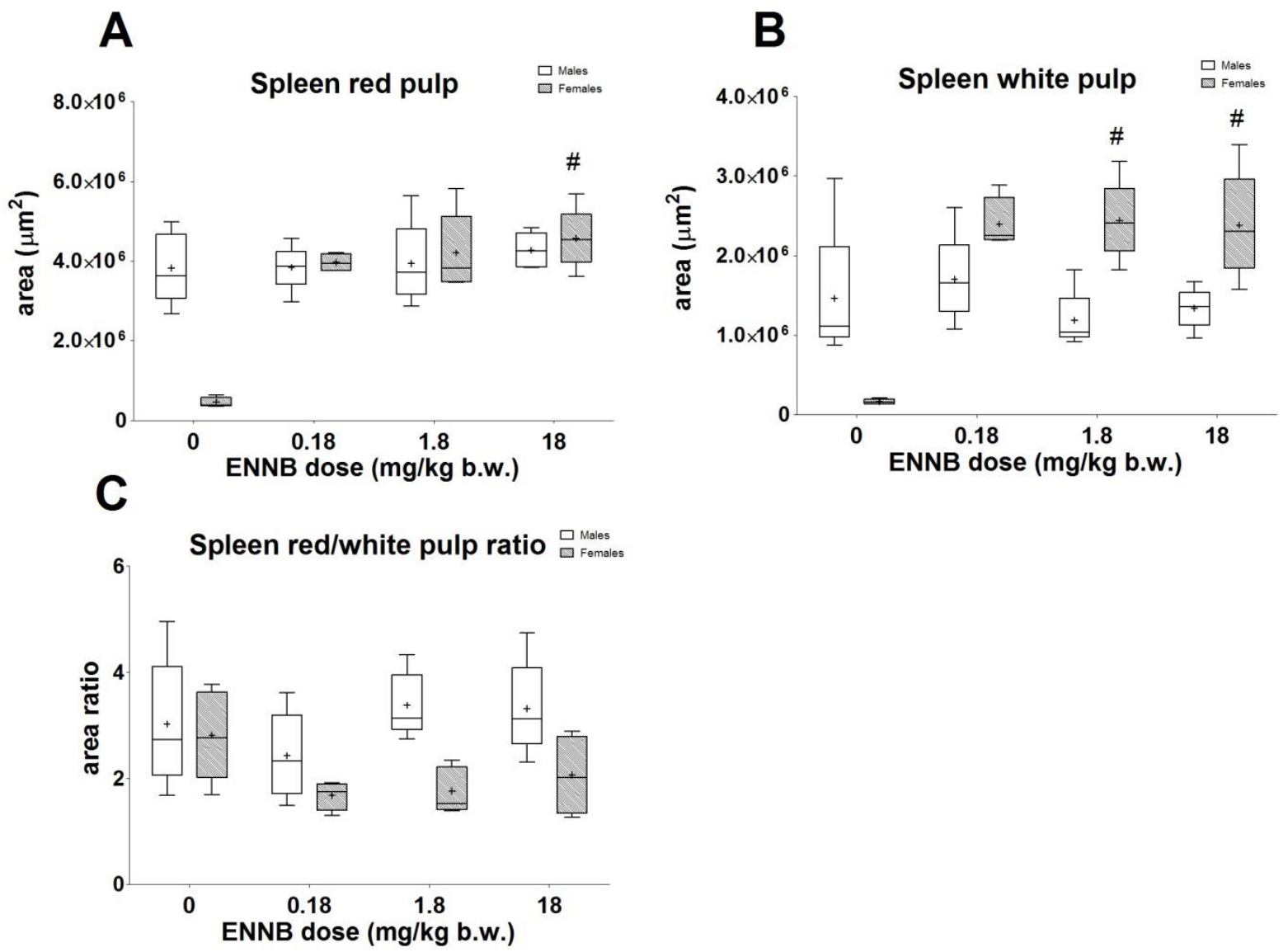

Figure 41: $\quad$ Histomorphometrical analysis of spleen red pulp area $(A)$ and white pulp area (B); ratio of the red to white areas $(\mathrm{C})$ in male (white box) and female (striped box) mice orally treated for 42 days with different doses of ENNB (0 - olive oil+DMSO6\%; 0.18, 1.8 and 18 $\mathrm{mg} / \mathrm{kg}$ per day). Levels are reported as interquartile box plots, with whiskers delimiting the 10 th and 90th percentiles. " + " indicates the mean value for each experimental group. \# $\mathrm{p}$ $<0.05$.

No histopathological effects were recorded in kidney of male and female mice (Table 25).

In thymus, no histopathological effect was observed (Table 25); however, histomorphometrical examination showed a significant increase of cortex area in female mice treated with ENNB1.8 and ENNB18; thymus medulla area was increased in female mice by ENNB18 (Fig. 42A-B). No effect was observed on thymus cortex/medulla ratio (Fig. 42C). 
A
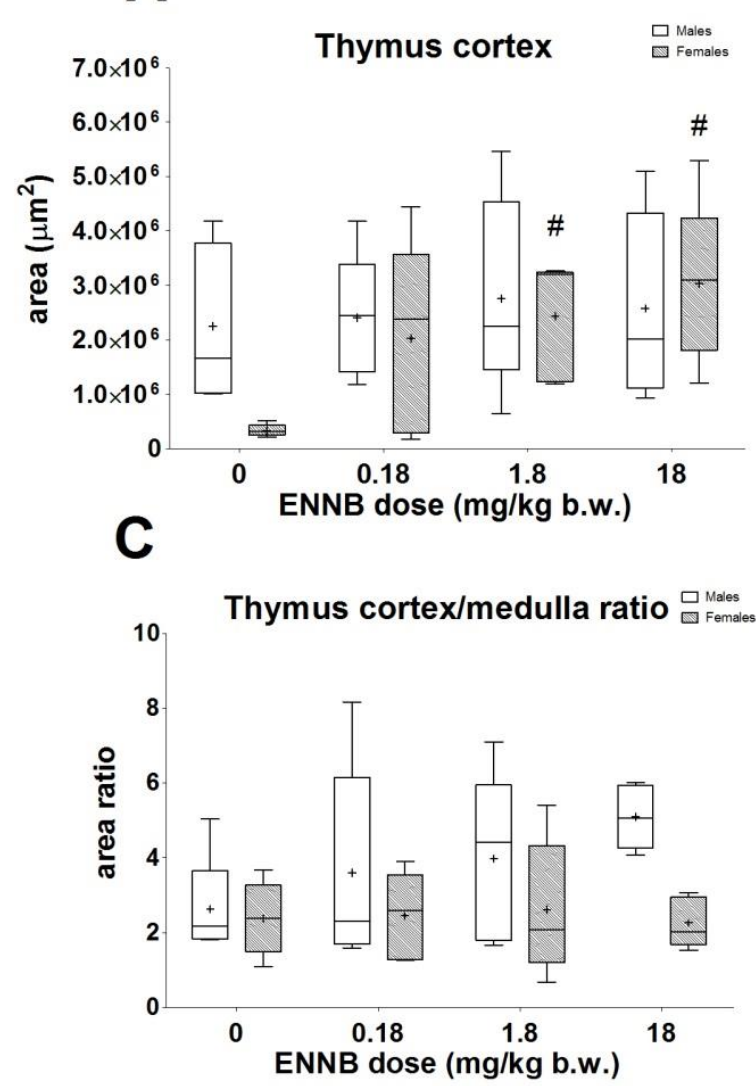

B

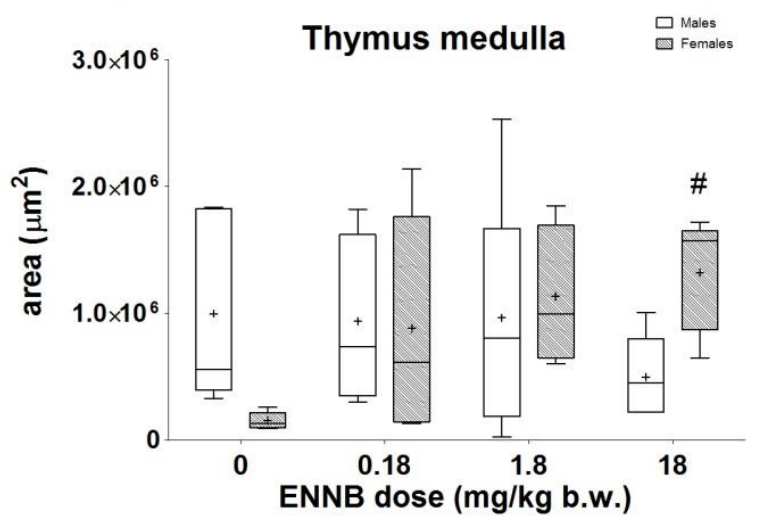

Figure 42: Histomorphometrical analysis of cortex (A) and medulla (B) thymus areas; ratio of the cortex to medulla areas (C) in male (white box) and female (striped box) mice orally treated for 42 days with different doses of ENNB (0 - olive oil+DMSO6\%; 0.18, 1.8 and 18 $\mathrm{mg} / \mathrm{kg}$ per day). Levels are reported as interquartile box plots, with whiskers delimiting the 10 th and 90th percentiles. " + " indicates the mean value for each experimental group. \# $\mathrm{p}$ $<0.05$.

No histopathological effects were observed in male and female adrenals (Table 25). However, both adrenal cortex and medulla areas were significantly increased by ENNB18 in female mice (Fig. 43A-B). The adrenal cortex/medulla ratio was unaffected by ENNB treatment (Fig. 43C). 
A
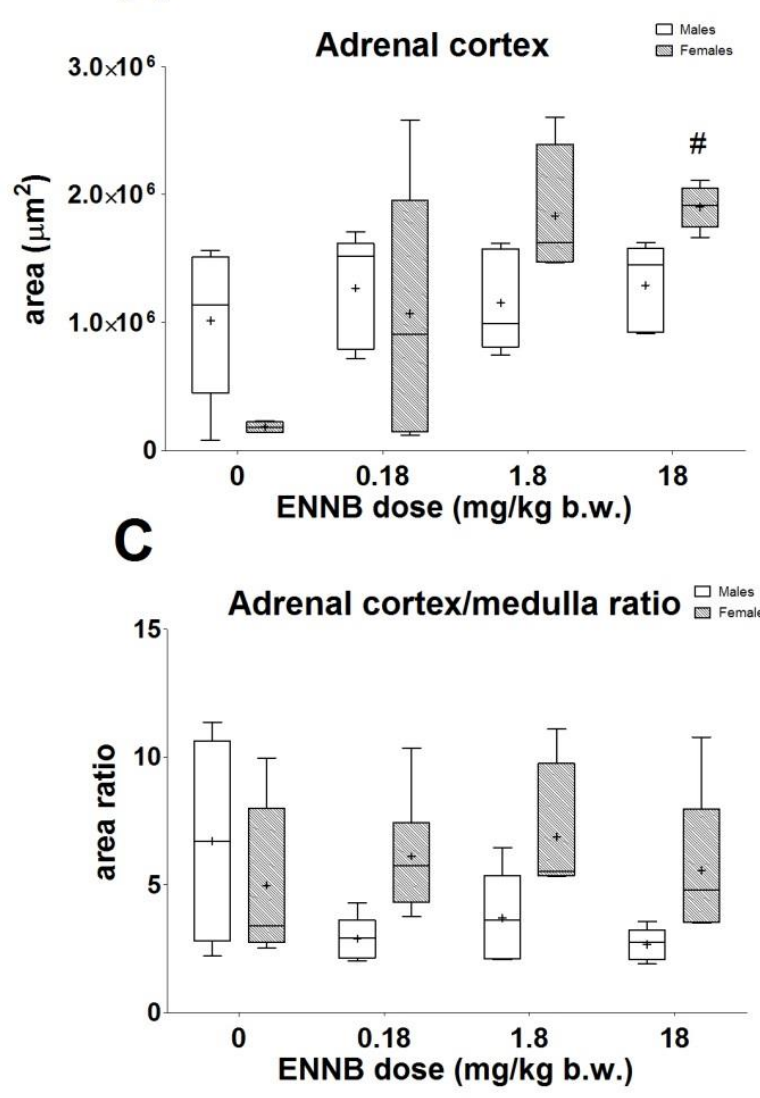

B

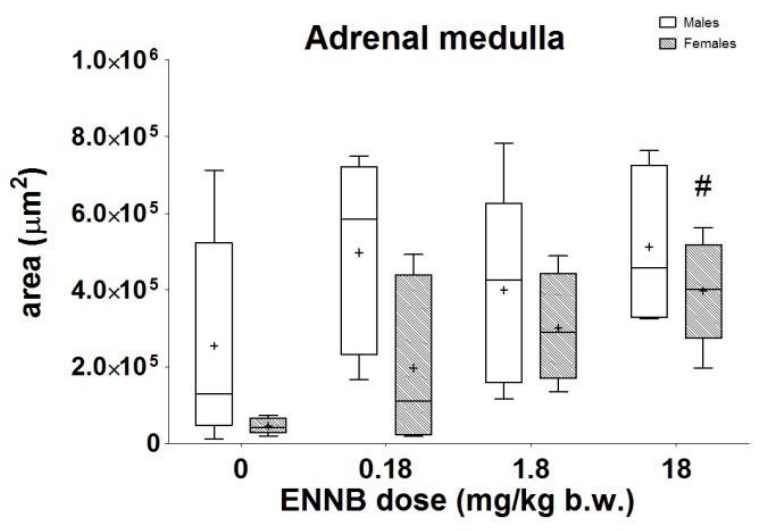

Figure 43: Histomorphometrical analysis of cortex (A) and medulla (B) adrenal areas; ratio of the cortex to medulla adrenal areas (C) in male (white box) and female (striped box) mice orally treated for 42 days with different doses of ENNB (0 - olive oil+DMSO6\%; $0.18,1.8$ and $18 \mathrm{mg} / \mathrm{kg}$ per day). Levels are reported as interquartile box plots, with whiskers delimiting the 10th and 90th percentiles. " + " indicates the mean value for each experimental group. \# $\mathrm{p}<0.05$.

In brain, no histopathological effect was observed in male and female mice (Table 25).

No histopathological effects were observed in female uteri (Table 25), whereas histomorphometrical measurement evidenced an increase in uterus endometrial and myometrial areas induced by ENNB1.8 and ENNB18 (Fig. 44A-B); moreover, luminal area was increased by ENNB1.8, the correlation with the treatment is unclear (Fig. 44C). 

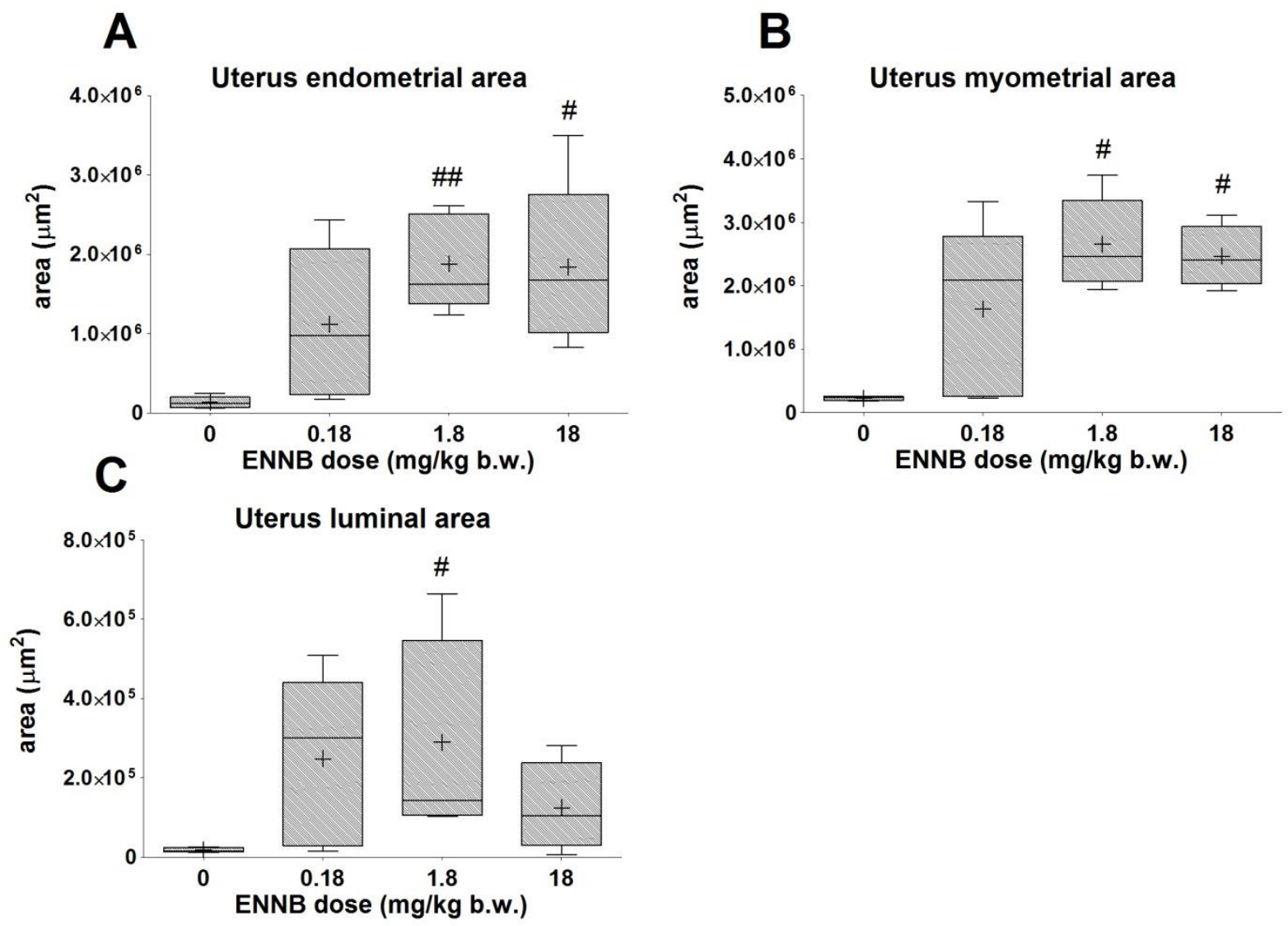

Figure 44: Histomorphometrical analysis of endometrial (A) myometrial (B) and luminal (C) uterus areas in female mice orally treated for 42 days with different doses of ENNB ( 0 olive oil+DMSO6\%; 0.18, 1.8 and $18 \mathrm{mg} / \mathrm{kg}$ per day). Levels are reported as interquartile box plots, with whiskers delimiting the 10th and 90th percentiles. "+" indicates the mean value for each experimental group. \# $p<0.05 ; \#$; $<0.01$.

Some ovaries were missed during tissue slide preparation. Thus, histopathological analysis was performed made only on the available samples. No histopathological alterations were observed (Table 25). No histomorphometrical analysis were performed.

Histomorphometrical measurement of testis areas did not evidence any significant alteration following ENNB treatment (Fig. 45A-E). 


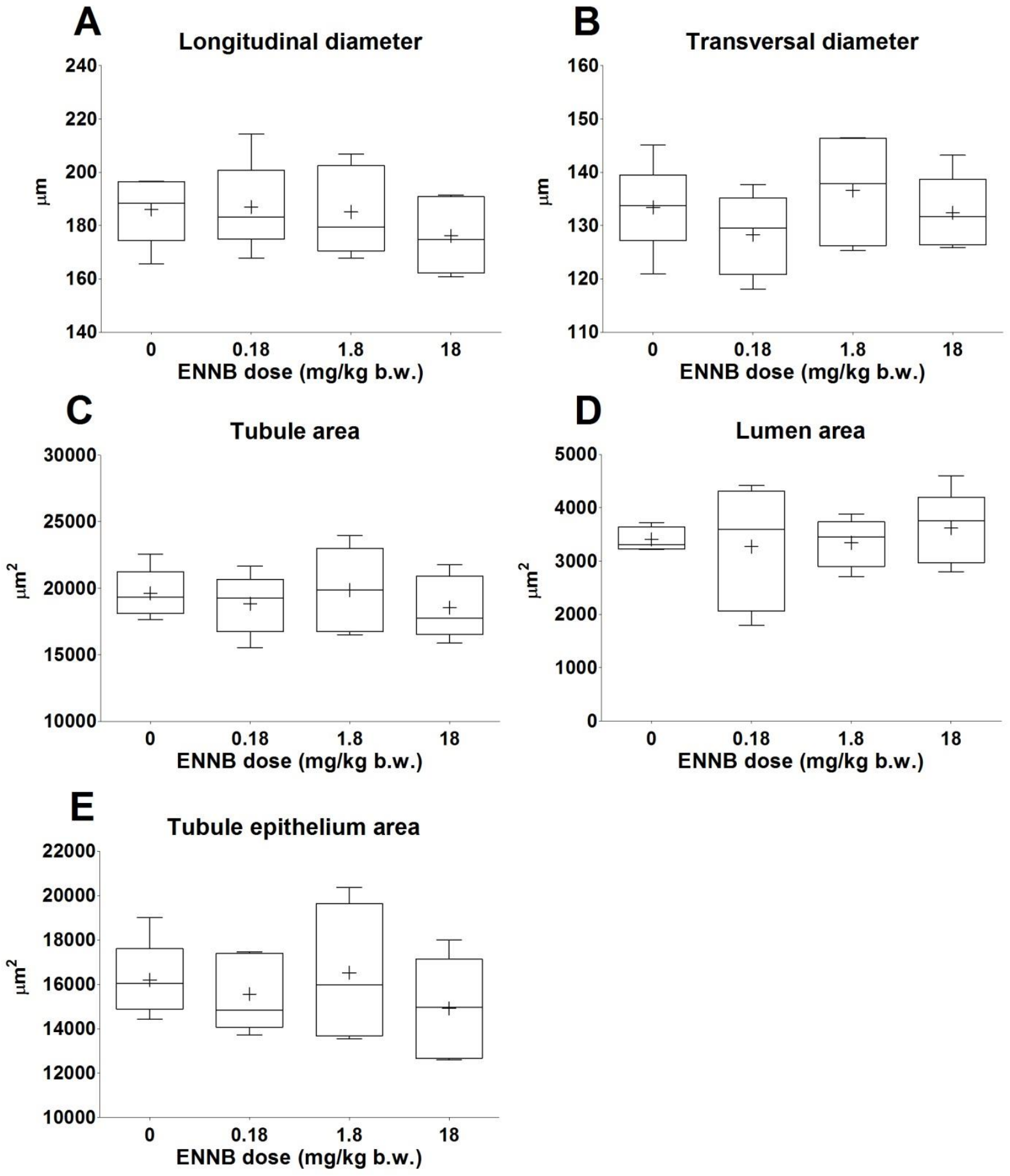

Figure 45: Histomorphometrical analysis of longitudinal $(A)$ and transversal $(B)$ testis diameter, testis tubule (C), lumen (D) and epithelium (E) areas in male mice orally treated for 42 days with different doses of ENNB ( 0 - olive oil+DMSO6\%; $0.18,1.8$ and $18 \mathrm{mg} / \mathrm{kg}$ per day). Levels are reported as interquartile box plots, with whiskers delimiting the 10th and 90th percentiles. " + " indicates the mean value for each experimental group. 


\subsection{8. $\quad$ ENNB - Biomarker endpoints}

\section{Liver and kidney serum biomarkers}

Biomarkers of liver function were not altered by ENNB treatment both in male and female mice (Fig. $46 \mathrm{~A}-\mathrm{B}$ ). On the other hand, CRE was significantly increased in males at ENNB0.18, the correlation with the treatment is unclear (Fig. 46C). No alterations were seen in female mice.
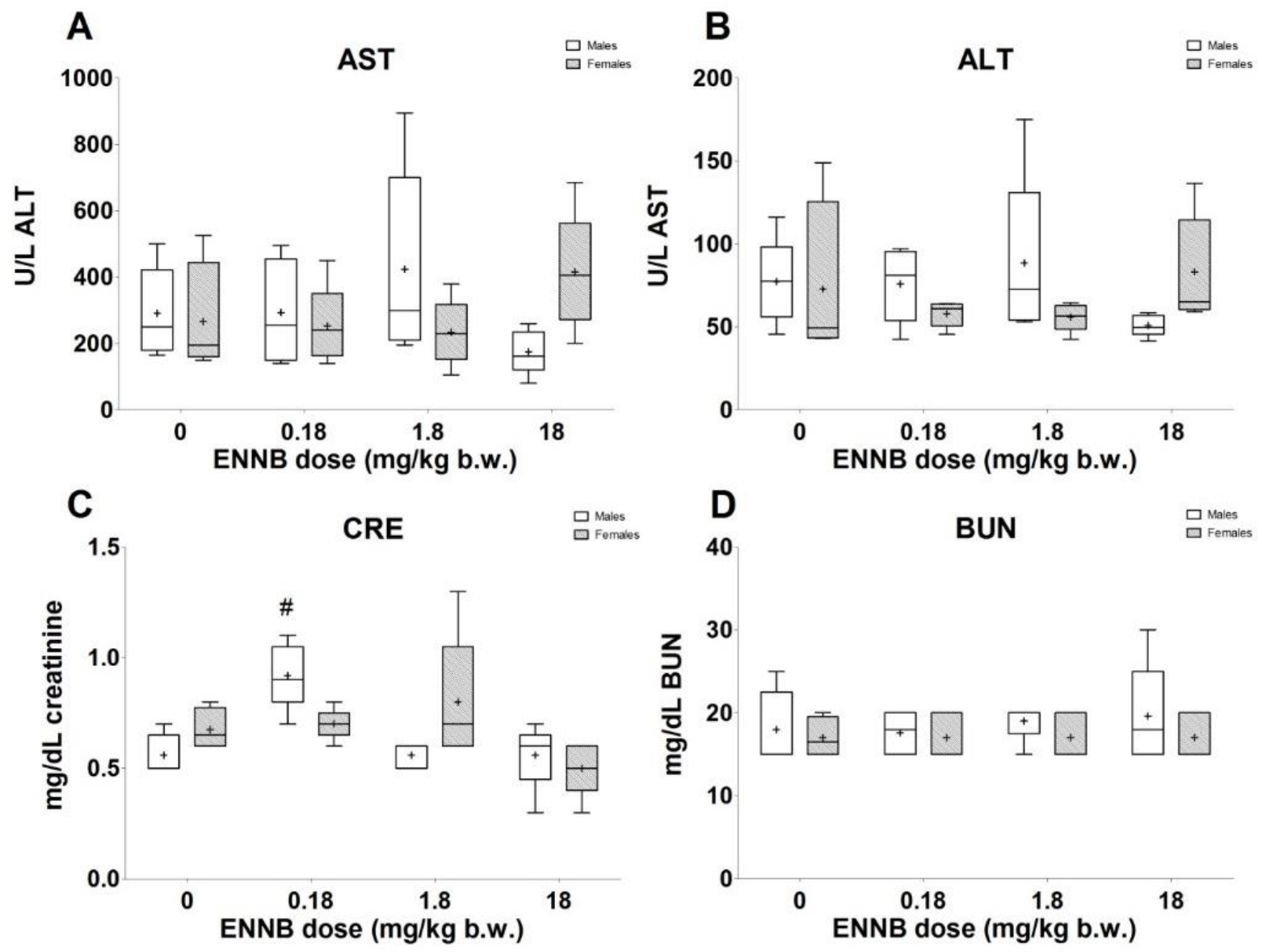

Figure 46: Serum levels of the liver biomarkers aspartate aminotransferase $(A)$ and alanine aminotransferase (B), and of the kidney biomarkers creatinine (C) and blood urea nitrogen (D) in male (white box) and female (striped box) mice orally treated for 42 days with different doses of ENNB ( 0 - olive oil+DMSO6\%; $0.18,1.8$ and $18 \mathrm{mg} / \mathrm{kg}$ per day). Levels are reported as interquartile box plots, with whiskers delimiting the 10th and 90th percentiles. " + " indicates the mean value for each experimental group. \# $p<0.05$.

\section{Hormone serum levels}

E2 was significantly decreased in male mice at ENNB18. No effects were seen in females (Fig. 47A).

Testosterone serum levels were not affected by ENNB treatment both in male and in female mice (Fig. 47B).

T4 serum levels were significantly decreased in females and significantly increased in males by ENNB18 (Fig. 47C).

No effect was recorded for TSH serum levels for both sexes (Fig. 47D). 

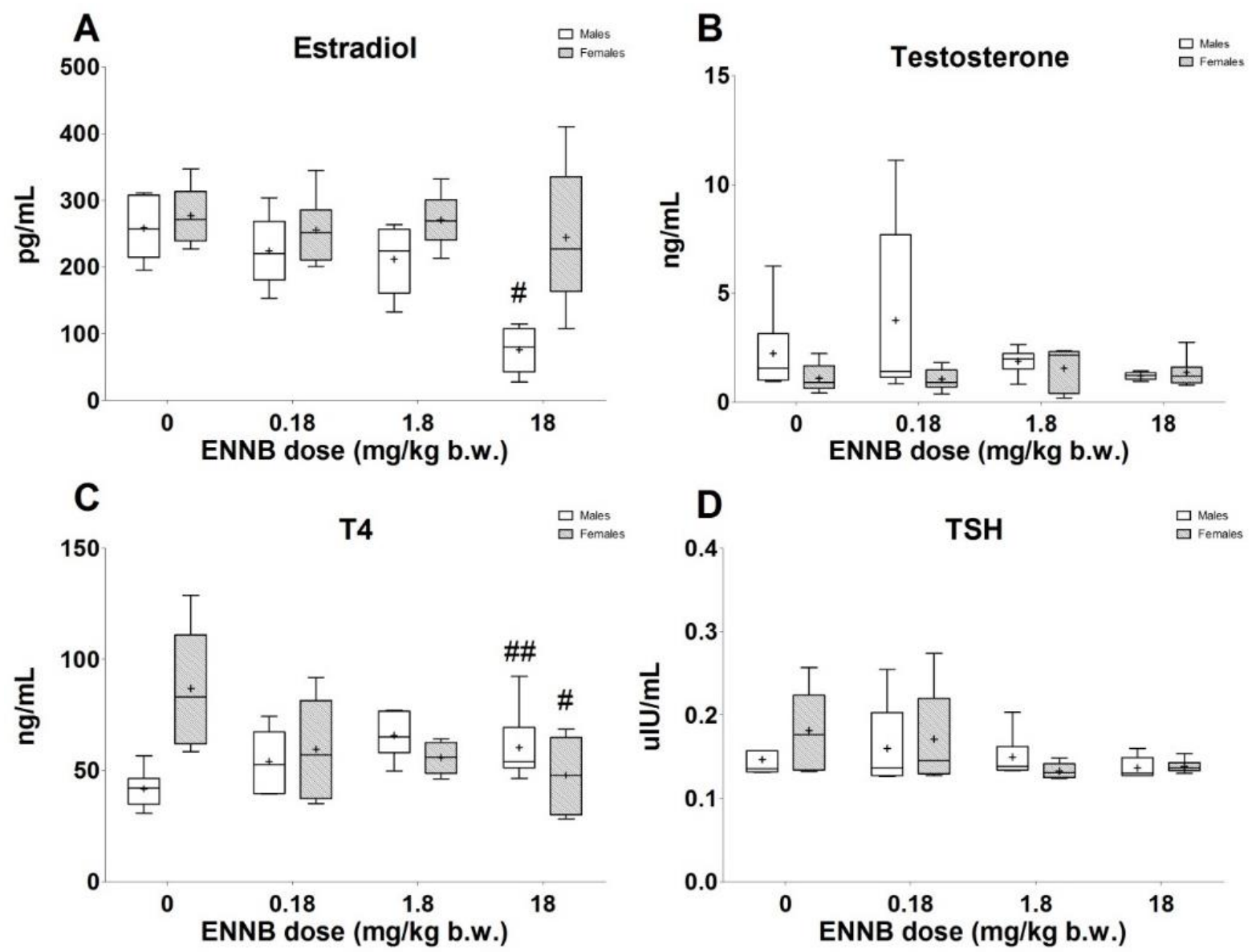

Figure 47: $\quad$ Serum levels of estradiol (A), testosterone (B), T4 (C) and TSH (D) in male (white box) and female (striped box) mice orally treated for 42 days with different doses of ENNB (0 - olive oil+DMSO6\%; $0.18,1.8$ and $18 \mathrm{mg} / \mathrm{kg}$ per day). Levels are reported as interquartile box plots, with whiskers delimiting the 10th and 90th percentiles. " + " indicates the mean value for each experimental group. \# $p<0.05 ; \# \#<<0.01$.

\section{Oxidative stress markers in brain}

The amount of ROS in brain was significantly increased in males at ENNB18 (Fig. 48A). Similarly, GSH level in brain was significantly increased in male and female mice at ENNB18 (Fig. 48B). 

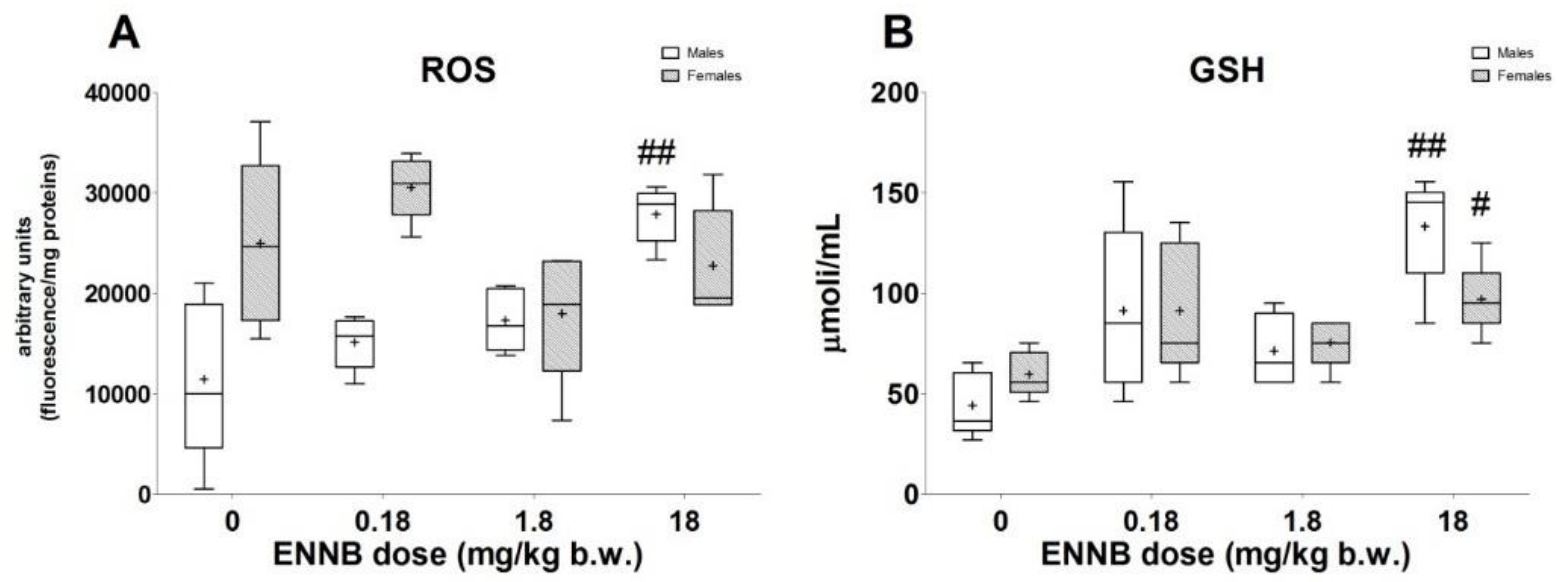

Figure 48: $\quad$ Brain levels of ROS (A) and GSH (B) in male (white box) and female (striped box) mice orally treated for 42 days with different doses of ENNB ( 0 - olive oil+DMSO6\%; 0.18 , 1.8 and $18 \mathrm{mg} / \mathrm{kg}$ per day). Levels are reported as interquartile box plots, with whiskers delimiting the 10th and 90th percentiles. "+" indicates the mean value for each experimental group. \# $\mathrm{p}<0.05 ; \# \# \mathrm{p}<0.01$.

\subsection{9. $\quad$ ENNB - Immunotoxicity endpoints}

\section{Lymphoid organ weights}

The results concerning lymphoid organs (spleen, thymus, mesenteric lymph node) were analyzed and reported together with all the organ weights in the "General Toxicity" section (Fig. 38; Table 24).

\section{Total serum antibodies}

ENNB treatment significantly affected antibodies levels only in female mice by increasing IgG at ENNB1.8 and ENN18 dose, and IgA at ENNB1.8 dose (Fig. 49). 
A

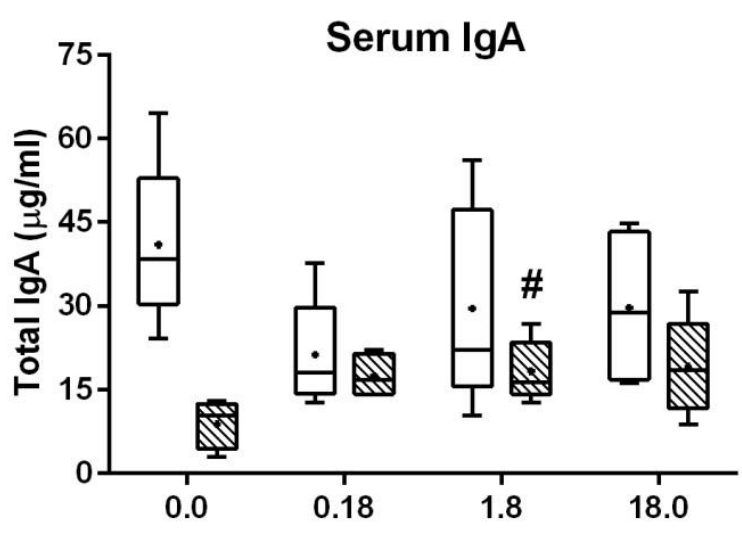

C ENNB dose (mg/kg b.w.)

\section{B}

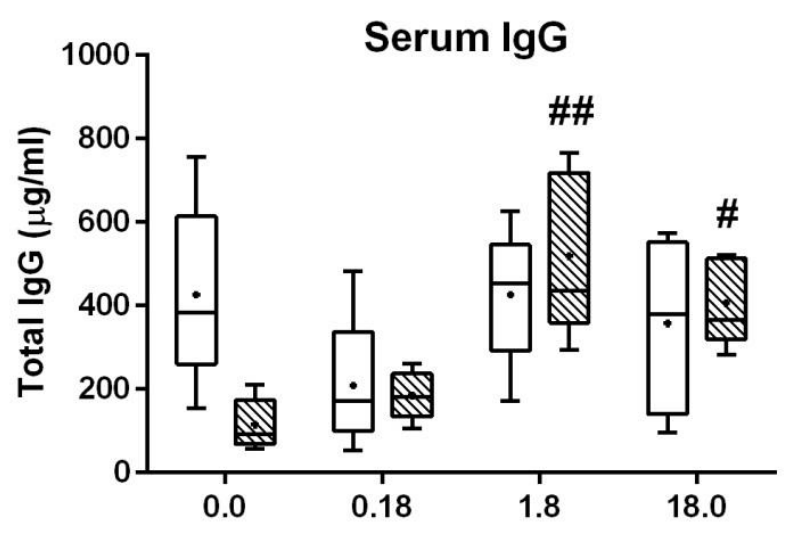

ENNB dose (mg/kg b.w.)

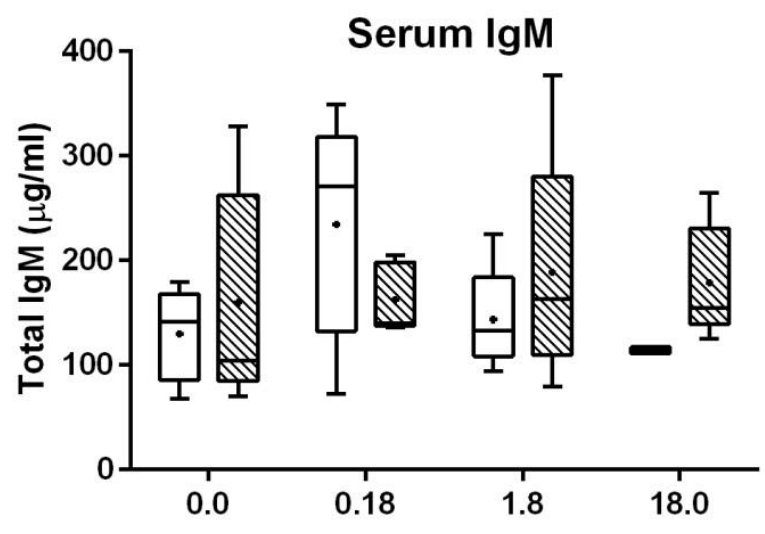

ENNB dose (mg/kg b.w.)

Figure 49: $\quad$ Serum antibody levels: total $\operatorname{IgA}(A), \operatorname{IgG}(B)$ and $\operatorname{Ig} M(C)$ in male (white box) and female (striped box) mice treated for 42 days orally with different doses of ENNB ( 0 - olive oil+DMSO6\%; 0.18, 1.8 and $18 \mathrm{mg} / \mathrm{kg}$ per day). Levels are reported as interquartile box plots, with whiskers delimiting the 10th and 90th percentiles. " + " indicates the mean value for each experimental group. \# $\mathrm{p}<0.05 ; \#$; $<0.01$.

\section{Blood count}

Statistically significant reductions were recorded for LYMPH and MID cells in both sexes after ENNB0.18 treatment (LYMPH) ENN18 (MID) (Fig. 50E-F). 
A

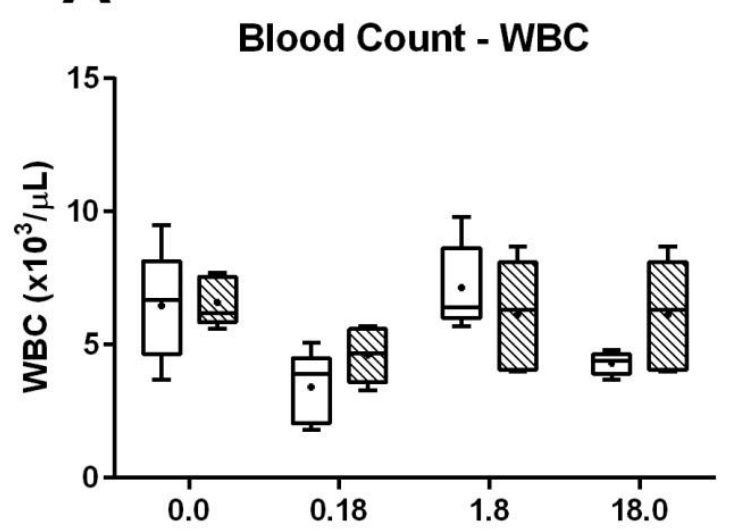

C ENNB dose (mg/kg b.w.)

Blood Count - PLT

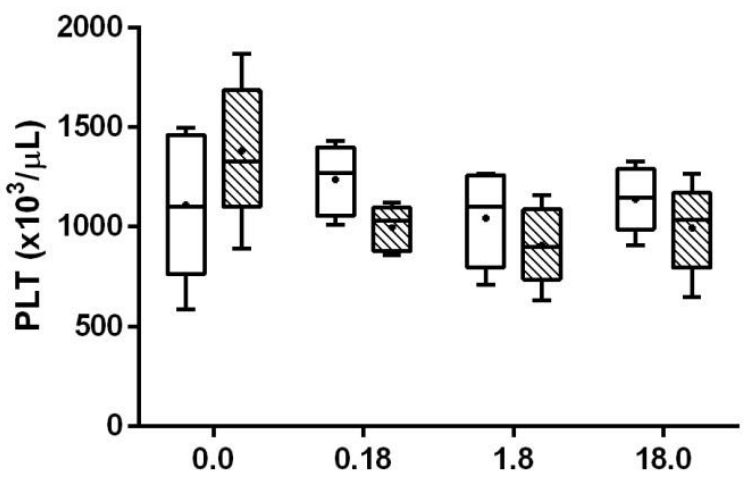

E ENNB dose (mg/kg b.w.)

Blood Count - LYMPH

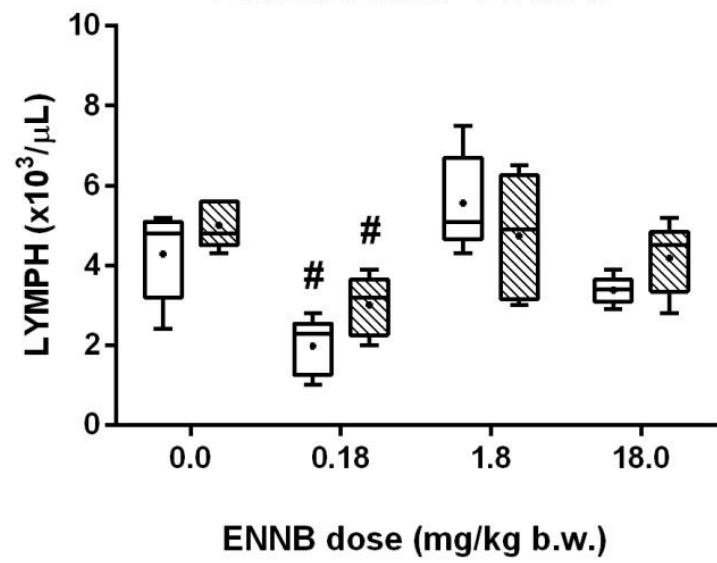

B

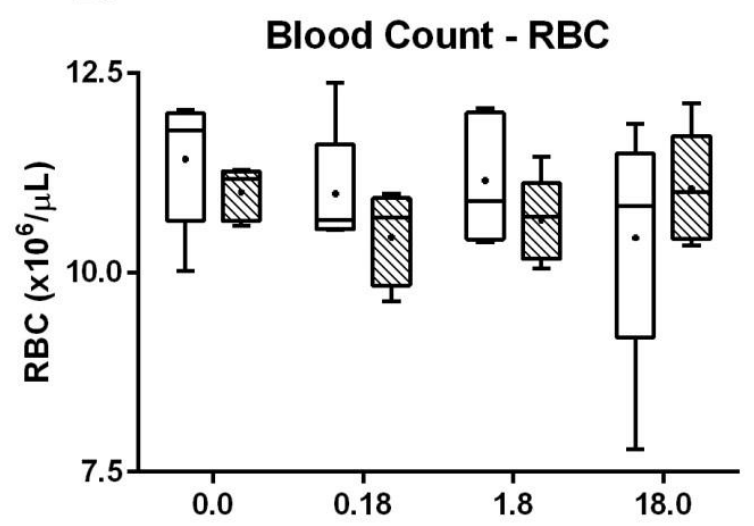

D ENNB dose (mg/kg b.w.)

Blood Count - GRAN

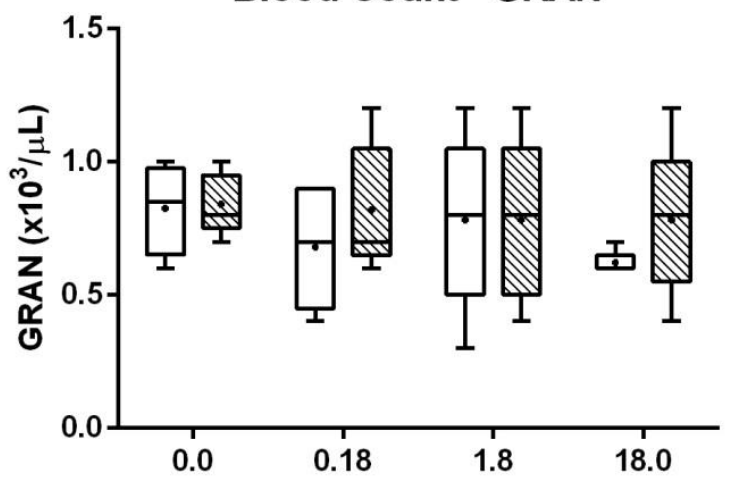

ENNB dose (mg/kg b.w.)

Blood Count - MID

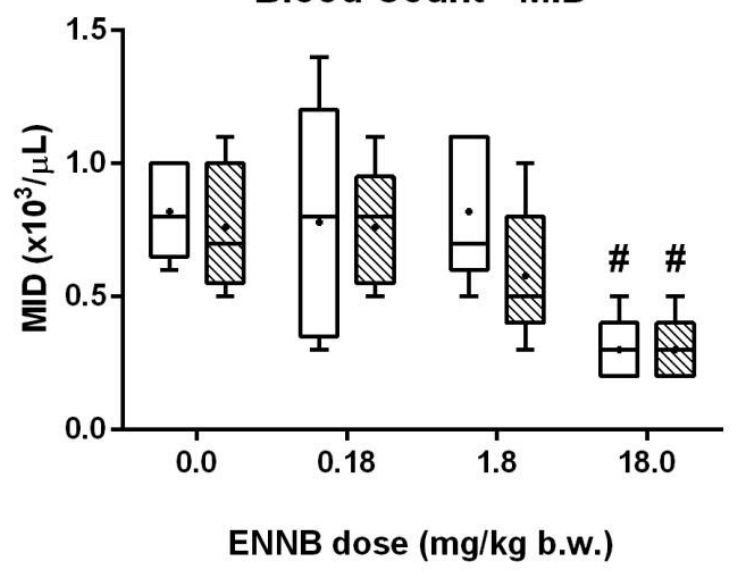

Figure 50: $\quad$ Blood count evaluation: WBC (A), RBC (B) and PLT (C) levels; among WBC, GRAN, (D), LYMPH (E) and rare MID (F) in male (white box) and female (striped box) mice orally treated for 42 days with different doses of ENNB ( 0 - olive oil+DMSO6\%; $0.18,1.8$ and 18 $\mathrm{mg} / \mathrm{kg}$ per day). Levels are reported as interquartile box plots, with whiskers delimiting the 10th and 90th percentiles. " + " indicates the mean value for each experimental group. \# $p$ $<0.05$. 


\section{NO production (spleen)}

No spontaneous NO production was observed in the absence of LPS stimulation. ENNB treatment did not induce significant effects in male mice, whereas both ENN1.8 and ENN18 significantly increased NO production in female mice (Fig. 51 and Table 26).

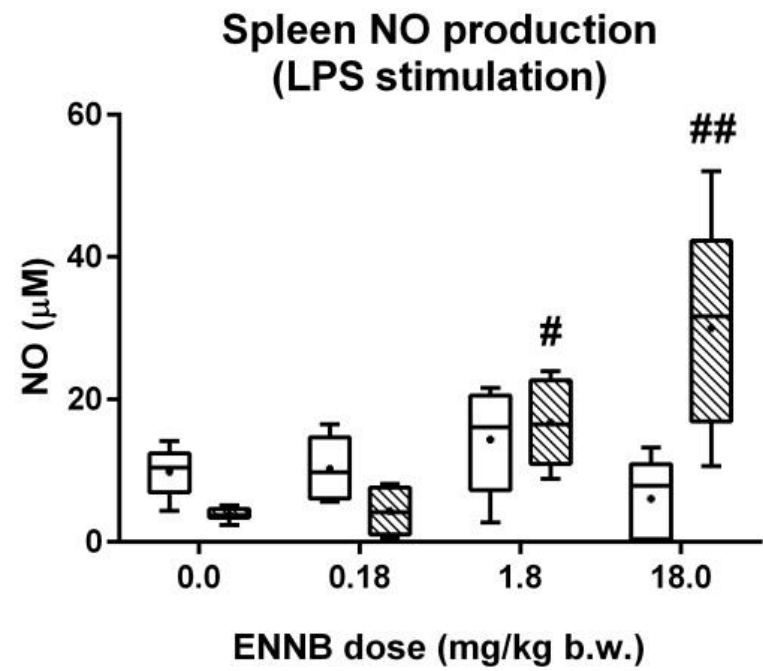

Figure 51: $\quad$ Nitric Oxide (NO) production after in vitro LPS stimulation of adherent spleen cells from male (white box) and female (striped box) mice orally treated for 42 days with different doses of ENNB (0 - olive oil+DMSO6\%; 0.18, 1.8 and $18 \mathrm{mg} / \mathrm{kg}$ per day). NO concentrations in culture supernatants are reported as interquartile box plots, with whiskers delimiting the 10th and 90th percentiles. " + " indicates the mean value for each experimental group. \# $\mathrm{p}<0.05, \# \# \mathrm{p}<0.01$.

Table 26: Nitric Oxide (NO) production after in vitro LPS stimulation of adherent spleen cells from male and female mice orally treated for 42 days with different doses of ENNB $(0.18,1.8$ and $18 \mathrm{mg} / \mathrm{kg}$ per day).

\begin{tabular}{|c|c|c|c|c|}
\hline & $\begin{array}{c}\text { Olive } \\
\text { oil+DMSO } \\
6 \%\end{array}$ & ENNB0.18 & ENNB1.8 & ENNB18 \\
\hline$M A L E$ & $\begin{array}{c}9.60 \\
10.43 \\
14.18 \\
4.38 \\
10.64\end{array}$ & $\begin{array}{c}5.74 \\
16.50 \\
9.77 \\
6.51 \\
12.83\end{array}$ & $\begin{array}{c}11.80 \\
16.13 \\
21.65 \\
19.48 \\
2.72\end{array}$ & $\begin{array}{c}0.71 \\
8.61 \\
7.89 \\
0.00 \\
13.27\end{array}$ \\
\hline$M E A N \pm S D$ & $9.85 \pm 3.52$ & $10.26 \pm 4.49$ & $14.36 \pm 7.49$ & $6.10 \pm 5.64$ \\
\hline$M E A N \pm S D$ & $\begin{array}{c}3.75 \\
2.37 \\
3.75 \\
3.75 \\
4.43 \\
5.12 \\
3.86 \pm 0.91\end{array}$ & $\begin{array}{l}8.17 \\
2.47 \\
0.63 \\
5.93\end{array}$ & $\begin{array}{c}23.98 \\
8.88 \\
16.53 \\
13.11 \\
21.36\end{array}$ & $\begin{array}{l}31.68 \\
32.51 \\
52.04 \\
10.70 \\
23.16\end{array}$ \\
\hline
\end{tabular}

(Values are reported as $\cdot M$ concentration of $\mathrm{NO}$ in culture supernatants) 


\section{Cytokine production (spleen)}

No spontaneous cytokine production was observed in the absence of stimulation. ENNB treatment did not affect IFNy levels in both sexes (Fig. 52A), whereas IL-10 production was significantly reduced only in male mice by ENNB1.8 (Fig. 52B).
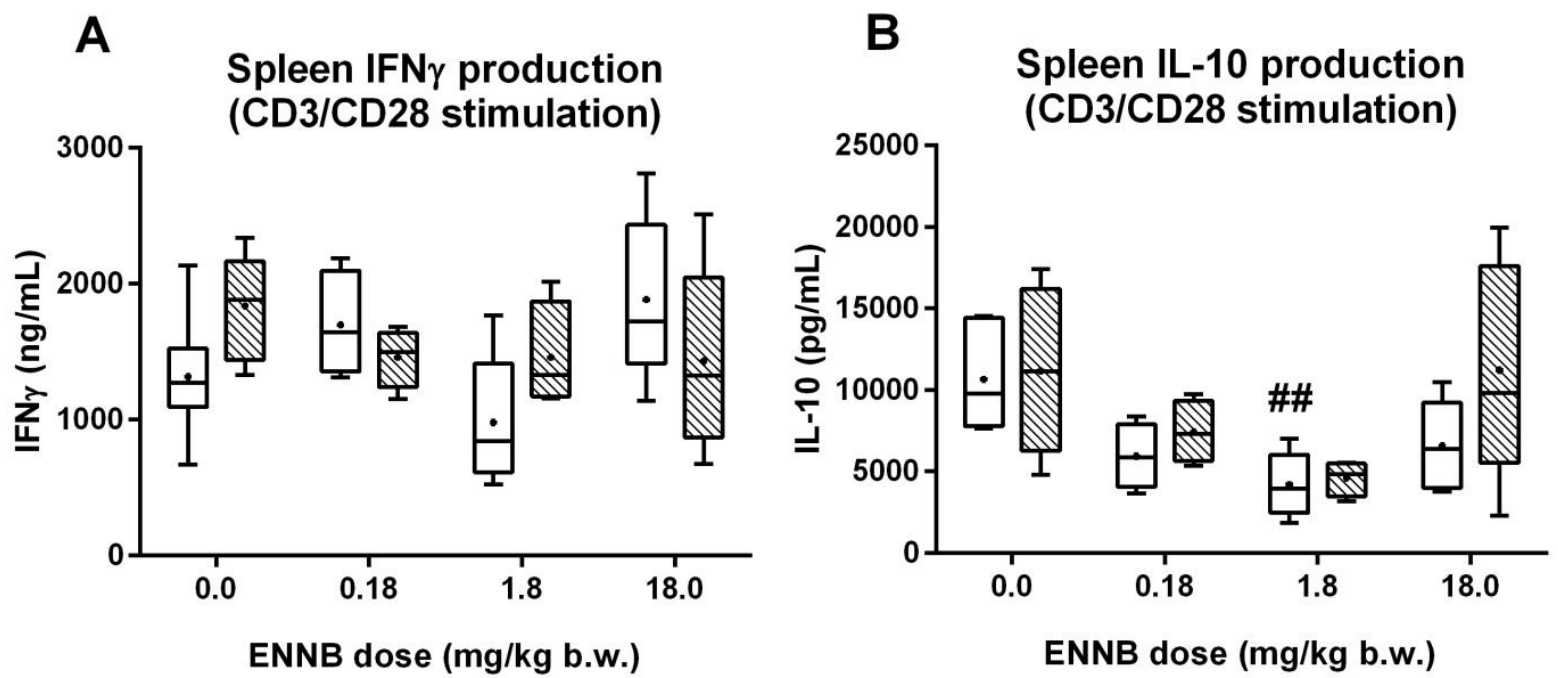

Figure 52: $\quad$ IFNY (A) and IL-10 (B) production after in vitro CD3/CD28 stimulation of spleen cells from male (white box) and female (striped box) mice orally treated for 42 days with different doses of ENNB (0 - olive oil+DMSO6\%; 0.18, 1.8 and $18 \mathrm{mg} / \mathrm{kg}$ per day). Cytokine concentrations in culture supernatants are as interquartile box plots, with whiskers delimiting the 10th and 90th percentiles. " + " indicates the mean value for each experimental group. \# $\mathrm{p}<0.01$.

\section{T lymphocyte (sub)populations (spleen)}

$T$ cell subpopulations in spleens are shown in Figure 53. ENNB treatment did not affect $T$ cell populations in female mice, whereas male mice presented a decreased $C D 3+/ C D 8+$ cell number at ENNB1.8 (Fig. 53E). 
A

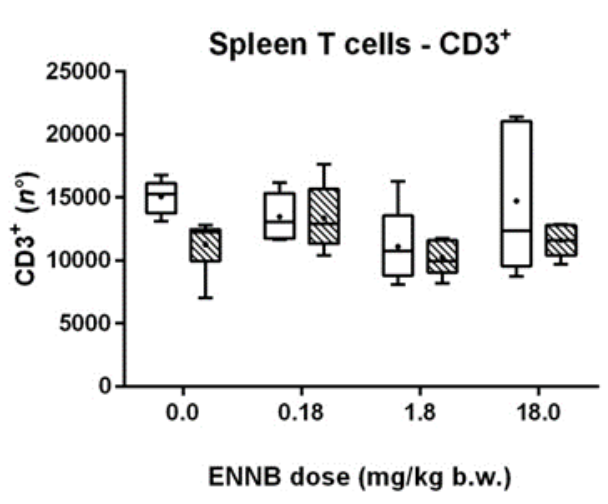

C

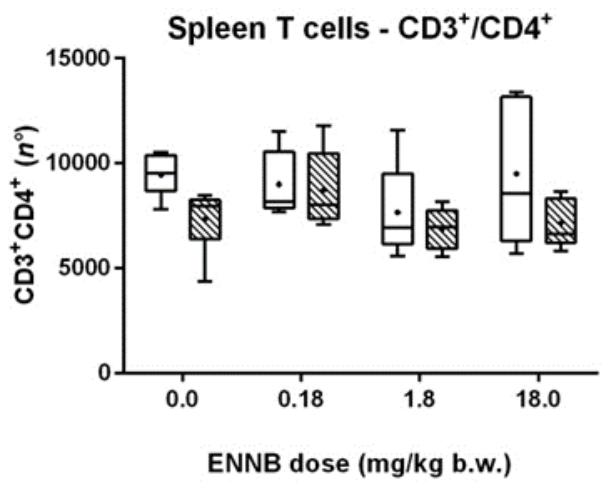

E

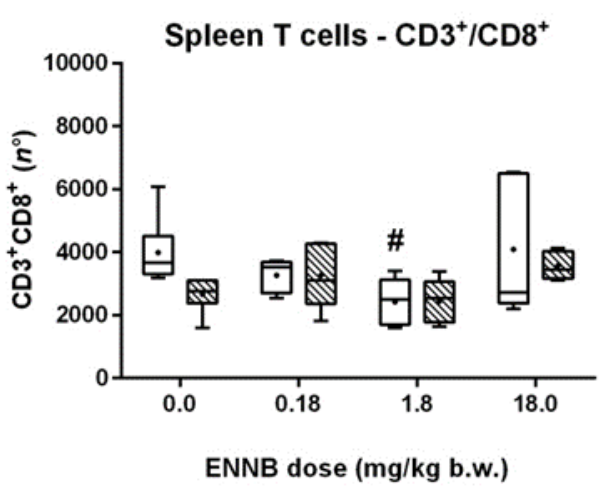

B

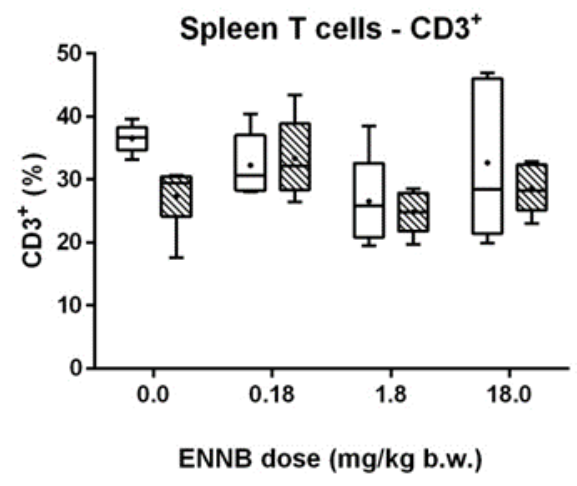

D

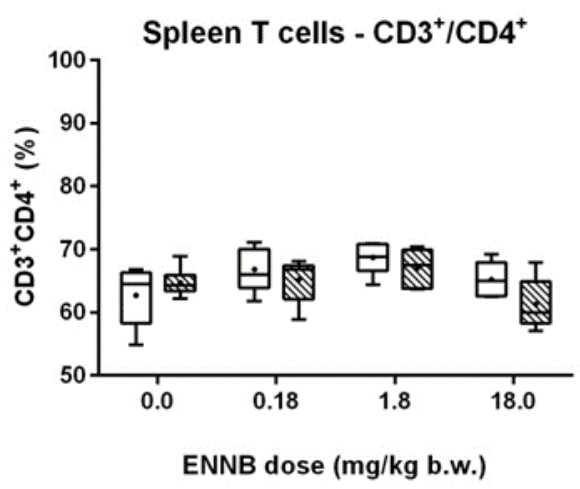

$\mathbf{F}$

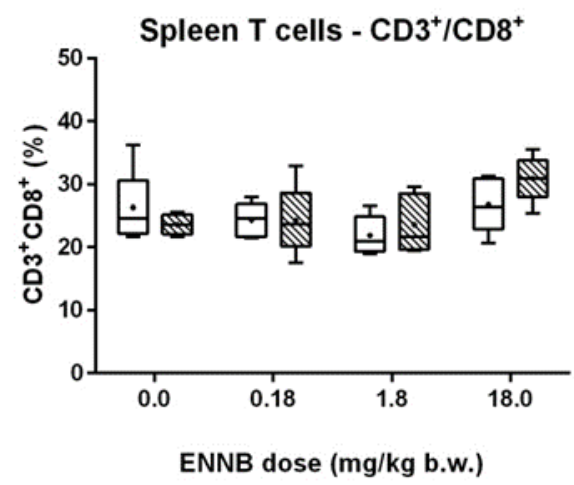

Figure 53: $\quad T$ cell subpopulations in spleens from male (white box) and female (striped box) mice orally treated for 42 days with different doses of ENNB ( 0 - olive oil+DMSO6\%; $0.18,1.8$ and $18 \mathrm{mg} / \mathrm{kg}$ per day), evaluated by cytofluorimetric phenotype analysis. Number and percentage of CD3-positive T cells $\left(n^{\circ} \mathrm{CD}^{+}, \mathrm{A} ; \% \mathrm{CD}^{+}, \mathrm{B}\right), \mathrm{CD} 3 / \mathrm{CD} 4$-double positive T cells $\left(n^{\circ} \mathrm{CD3}^{+} / \mathrm{CD}^{+}, \mathrm{C} ; \% \mathrm{CD}^{+} / \mathrm{CD}^{+}, \mathrm{D}\right), \mathrm{CD} 3 / \mathrm{CD} 8$-double positive $\mathrm{T}$ cells $\left(n^{\circ}\right.$ $\mathrm{CD}^{+} / \mathrm{CD}^{+}, \mathrm{E} ; \% \mathrm{CD}^{+} / \mathrm{CD}^{+}, \mathrm{F}$ ), are reported as interquartile box plots, with whiskers delimiting the $10^{\text {th }}$ and $90^{\text {th }}$ percentiles. " + " indicates the mean value for each experimental group. $\# \mathrm{p}<0.05$. 


\subsubsection{0. $\quad$ ENNB - Genotoxicity endpoints}

\section{Comet assay}

In male mice, the Comet assay results collected on all organs are summarized in Table 27 and Figure 54. Data show that treatment with ENNB did not significantly increase the \%TI over the values of the CTRL group (olive oil + DMSO6\%) in any of the organs analyzed. Here and in all the following tables, data relative to the positive CTRL groups are those reported in the corresponding tables relative to experiments with BEA.

Table 27: Alkaline Comet assay in different organs/tissues of male mice after repeated oral treatments with different doses of ENNB ( 0 - olive oil+DMSO6\%; $0.18,1.8$ and $18 \mathrm{mg} / \mathrm{kg}$ per day). For each experimental group the mean of median and standard error of the values obtained from each animal for tail length (Tail-L), tail intensity (\%TI) and Olive Tail Moment (OTM) are reported. The mean \% hedgehogs for each group is shown.

\begin{tabular}{llcccc}
\hline Tissue/organ & \multicolumn{1}{c}{ Treatment } & $\begin{array}{c}\text { Tail-L } \\
\text { mean } \pm \text { SE }\end{array}$ & $\begin{array}{c}\text { \% TI } \\
\text { mean } \pm \text { SE }\end{array}$ & $\begin{array}{c}\text { OTM } \\
\text { mean } \pm \text { SE }\end{array}$ & $\begin{array}{c}\text { \% hedgehogs } \\
\text { mean } \pm \text { SE }\end{array}$ \\
\hline Blood & olive oil + DMSO6\% & $0.613 \pm 0.195$ & $0.343 \pm 0.049$ & $0.025 \pm 0.006$ & $0.127 \pm 0.127$ \\
& ENNB0.18 & $0.194 \pm 0.104$ & $0.203 \pm 0.051$ & $0.004 \pm 0.002$ & $0.245 \pm 0.150$ \\
& ENNB1.8 & $0.758 \pm 0.378$ & $0.311 \pm 0.093$ & $0.047 \pm 0.037$ & $0.362 \pm 0.237$ \\
& ENNB18 & $0.161 \pm 0.088$ & $0.230 \pm 0.017$ & $0.009 \pm 0.003$ & $0.127 \pm 0.127$ \\
\hline Liver & olive oil + DMSO6\% & $1.306 \pm 0.306$ & $0.213 \pm 0.045$ & $0.049 \pm 0.018$ & $2.584 \pm 0.724$ \\
& ENNB0.18 & $1.677 \pm 0.400$ & $0.239 \pm 0.092$ & $0.078 \pm 0.041$ & $2.127 \pm 0.414$ \\
& ENNB1.8 & $1.032 \pm 0.355$ & $0.164 \pm 0.041$ & $0.041 \pm 0.019$ & $1.624 \pm 0.876$ \\
& ENNB18 & $1.258 \pm 0.324$ & $0.209 \pm 0.070$ & $0.042 \pm 0.018$ & $0.945 \pm 0.400$ \\
\hline Kidney & olive oil + DMSO6\% & $1.613 \pm 0.338$ & $0.342 \pm 0.072$ & $0.092 \pm 0.025$ & $2.675 \pm 0.874$ \\
& ENNB0.18 & $1.645 \pm 0.454$ & $0.323 \pm 0.083$ & $0.089 \pm 0.032$ & $2.522 \pm 1.070$ \\
& ENNB1.8 & $1.290 \pm 0.265$ & $0.297 \pm 0.057$ & $0.061 \pm 0.017$ & $2.817 \pm 0.686$ \\
& ENNB18 & $0.984 \pm 0.166$ & $0.197 \pm 0.025$ & $0.039 \pm 0.007$ & $1.340 \pm 0.230$ \\
\hline Duodenum & olive oil + DMSO6\% & $1.371 \pm 0.692$ & $0.439 \pm 0.283$ & $0.099 \pm 0.077$ & $13.423 \pm 3.969$ \\
& ENNB0.18 & $0.952 \pm 0.370$ & $0.402 \pm 0.179$ & $0.077 \pm 0.044$ & $11.212 \pm 3.449$ \\
& ENNB1.8 & $1.597 \pm 0.181$ & $0.479 \pm 0.088$ & $0.095 \pm 0.023$ & $15.417 \pm 3.683$ \\
& ENNB18 & $0.903 \pm 0.276$ & $0.164 \pm 0.020$ & $0.022 \pm 0.008$ & $6.267 \pm 0.472$ \\
\hline Testis & olive oil + DMSO6\% & $0.468 \pm 0.229$ & $0.241 \pm 0.033$ & $0.017 \pm 0.014$ & $0.588 \pm 0.315$ \\
& ENNB0.18 & $0.549 \pm 0.150$ & $0.204 \pm 0.035$ & $0.014 \pm 0.005$ & $1.522 \pm 0.375$ \\
& ENNB1.8 & $0.855 \pm 0.225$ & $0.272 \pm 0.047$ & $0.035 \pm 0.013$ & $1.009 \pm 0.312$ \\
& ENNB18 & $0.694 \pm 0.194$ & $0.243 \pm 0.035$ & $0.027 \pm 0.010$ & $0.505 \pm 0.309$ \\
\hline
\end{tabular}



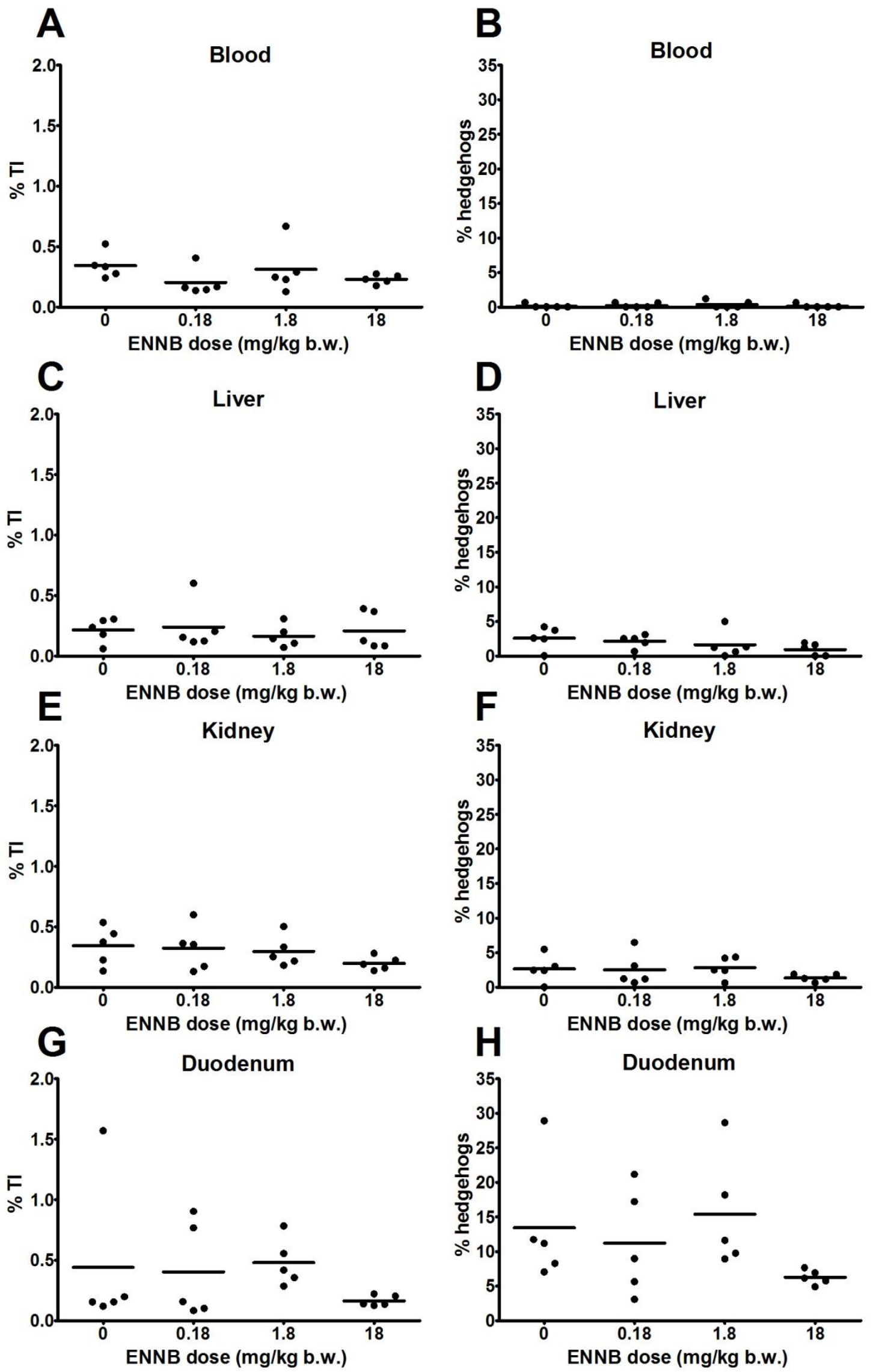

The present document has been produced and adopted by the bodies identified above as authors. In accordance with Article 36 of Regulation (EC) No $178 / 2002$, this task has been carried out exclusively by the authors in the context of a grant agreement between the European Food Safety Authority and the authors. The present document is published complying with the transparency principle to which the Authority is subject. It cannot be considered as an output adopted by the Authority. The European Food Safety Authority reserves its rights, view and position as regards the issues addressed and the conclusions reached in the present document, without prejudice to the rights of the authors. 

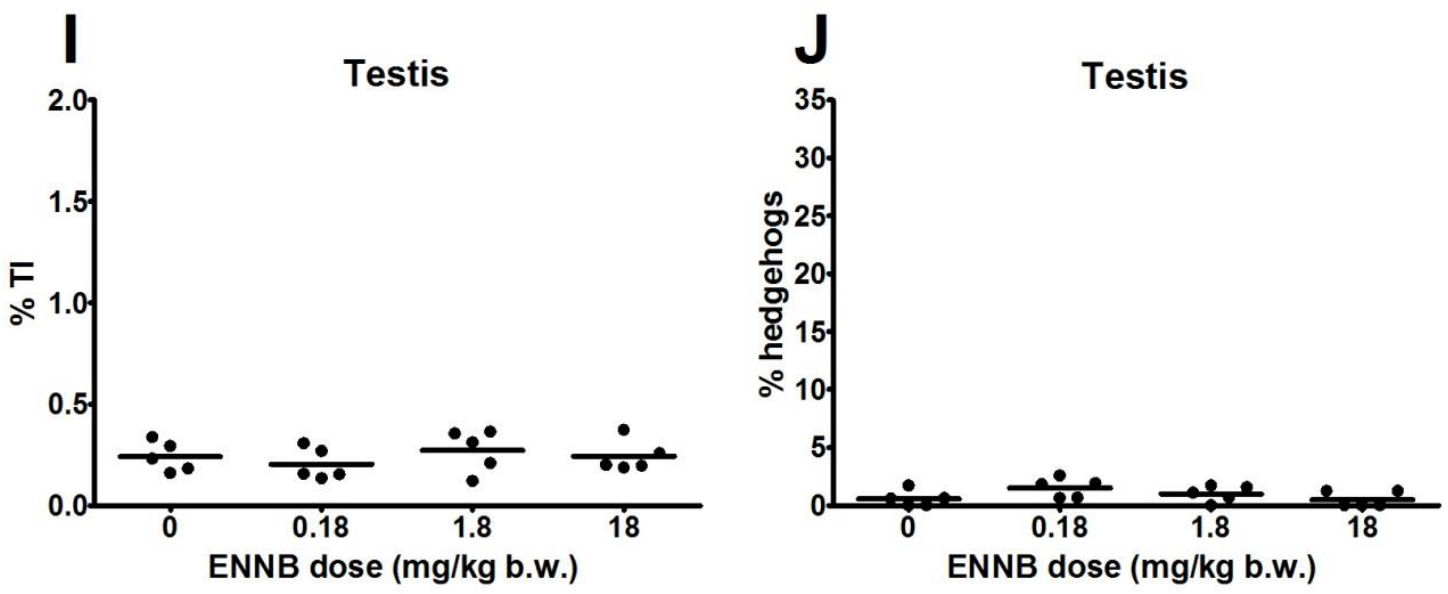

Figure 54: $\quad$ Alkaline Comet assay in different organs/tissues of male mice after repeated oral treatments with different doses of ENNB ( 0 - olive oil+DMSO6\%; $0.18,1.8$ and $18 \mathrm{mg} / \mathrm{kg}$ per day). In $A, C, E, G$ and I dots represent the median of tail intensity obtained for each animal and lines represent the mean of tail intensity for each group. In B, D, F, H and J dots represent the percentage of hedgehogs obtained for each mouse and lines are the mean for each group.

ENNB treatment did not induce any increase of tail intensity in epididymal spermatozoa, analyzed by neutral Comet assay (Figure 55).

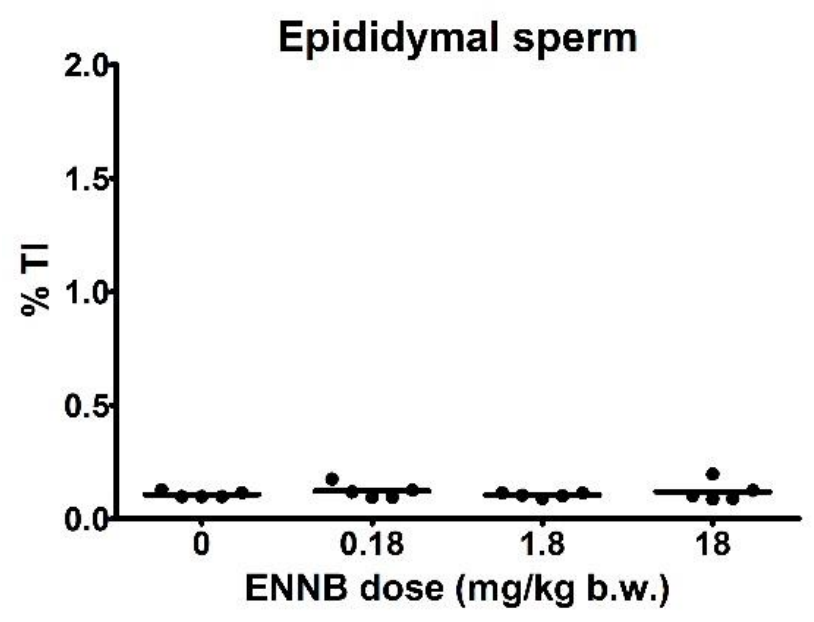

Figure 55: Neutral Comet assay in epididymal spermatozoa of male mice after repeated oral treatments with different doses of ENNB ( 0 - olive oil+DMSO6\%; $0.18,1.8$ and $18 \mathrm{mg} / \mathrm{kg}$ per day). Dots represent the median of \%TI obtained for each animal and lines represent the mean of \%TI for each group.

In female mice the DNA damage measured by alkaline Comet assay after ENNB is represented in Table 28 and in Figure 56. The slides of negative CTRL of blood were irreversibly damaged, so results of treated groups could not be compared to a negative CTRL. For this reason no assessment of DNA strand breaks in blood cells could be performed. As regards the other organs, ENNB affected kidney by a significant decrease of tail length at $0.18 \mathrm{mg} / \mathrm{kg}$. In the same organ a significant increase of $\%$ hedgehogs was observed at $1.8 \mathrm{mg} / \mathrm{kg}$. No effect was observed in liver, ovary and duodenum. 
Table 28: Alkaline Comet assay in different organs/tissues of female mice after repeated oral treatments with different doses of ENNB ( 0 - olive oil+DMSO6\%; $0.18,1.8$ and $18 \mathrm{mg} / \mathrm{kg}$ per day). For each experimental group the mean of median and standard error of the values obtained from each animal for tail length (Tail-L), tail intensity (\%TI) and Olive Tail Moment (OTM) are reported. The mean \% hedgehogs for each group is shown.

\begin{tabular}{llcccc}
\hline Tissue/organ & \multicolumn{1}{c}{ Treatment } & $\begin{array}{c}\text { Tail-L } \\
\text { mean } \pm \text { SE }\end{array}$ & $\begin{array}{c}\text { \% TI } \\
\text { mean } \pm \text { SE }\end{array}$ & $\begin{array}{c}\text { OTM } \\
\text { mean } \pm \text { SE }\end{array}$ & $\begin{array}{c}\text { \% hedgehogs } \\
\text { mean } \pm \text { SE }\end{array}$ \\
\hline Blood & olive oil + DMSO6\% & & \multicolumn{2}{c}{ Not analyzable } \\
& ENNB0.18 & $0.092 \pm 0.043$ & $0.253 \pm 0.027$ & $0.023 \pm 0.003$ & $0.000 \pm 0.000$ \\
& ENNB1.8 & $0.046 \pm 0.028$ & $0.581 \pm 0.081$ & $0.042 \pm 0.004$ & $0.000 \pm 0.000$ \\
& ENNB18 & $0.092 \pm 0.043$ & $0.359 \pm 0.085$ & $0.026 \pm 0.006$ & $0.000 \pm 0.000$ \\
\hline Liver & olive oil + DMSO6\% & $1.582 \pm 0.247$ & $0.370 \pm 0.060$ & $0.043 \pm 0.008$ & $2.133 \pm 0.800$ \\
& ENNB0.18 & $0.954 \pm 0.302$ & $0.226 \pm 0.036$ & $0.023 \pm 0.005$ & $0.933 \pm 0.653$ \\
& ENNB1.8 & $0.583 \pm 0.156$ & $0.163 \pm 0.036$ & $0.020 \pm 0.004$ & $1.600 \pm 0.266$ \\
& ENNB18 & $2.337 \pm 0.905$ & $0.825 \pm 0.425$ & $0.091 \pm 0.049$ & $2.133 \pm 0.975$ \\
\hline Kidney & olive oil + DMSO6\% & $1.582 \pm 0.520$ & $0.749 \pm 0.335$ & $0.071 \pm 0.032$ & $0.267 \pm 0.163$ \\
& ENNB0.18 & $0.419 \pm 0.172 \#$ & $0.197 \pm 0.047$ & $0.017 \pm 0.003$ & $0.133 \pm 0.133$ \\
& ENNB1.8 & $0.490 \pm 0.158$ & $0.160 \pm 0.043$ & $0.017 \pm 0.005$ & $2.000 \pm 0.558 \# \#$ \\
& ENNB18 & $0.722 \pm 0.213$ & $0.220 \pm 0.044$ & $0.020 \pm 0.005$ & $1.333 \pm 0.298$ \\
\hline Duodenum & olive oil + DMSO6\% & $2.001 \pm 0.174$ & $1.384 \pm 0.080$ & $0.129 \pm 0.006$ & $1.868 \pm 0.389$ \\
& ENNB0.18 & $1.514 \pm 0.272$ & $1.300 \pm 0.175$ & $0.117 \pm 0.016$ & $1.070 \pm 0.343$ \\
& ENNB1.8 & $1.421 \pm 0.543$ & $1.170 \pm 0.438$ & $0.115 \pm 0.045$ & $6.674 \pm 2.622$ \\
& ENNB18 & $1.141 \pm 0.284$ & $1.038 \pm 0.227$ & $0.092 \pm 0.021$ & $3.209 \pm 1.252$ \\
\hline Ovary & olive oil + DMSO6\% & $0.663 \pm 0.339$ & $0.537 \pm 0.353$ & $0.049 \pm 0.032$ & $0.802 \pm 0.133$ \\
& ENNB0.18 & $0.326 \pm 0.157$ & $0.226 \pm 0.050$ & $0.020 \pm 0.004$ & $0.800 \pm 0.389$ \\
& ENNB1.8 & $0.281 \pm 0.087$ & $0.142 \pm 0.014$ & $0.013 \pm 0.001$ & $2.000 \pm 0.789$ \\
& ENNB18 & $0.580 \pm 0.437$ & $0.256 \pm 0.117$ & $0.025 \pm 0.013$ & $1.381 \pm 0.734$ \\
\hline
\end{tabular}

$\# p<0.05 ;{ }^{\#} \mathrm{p}<0.01$ 

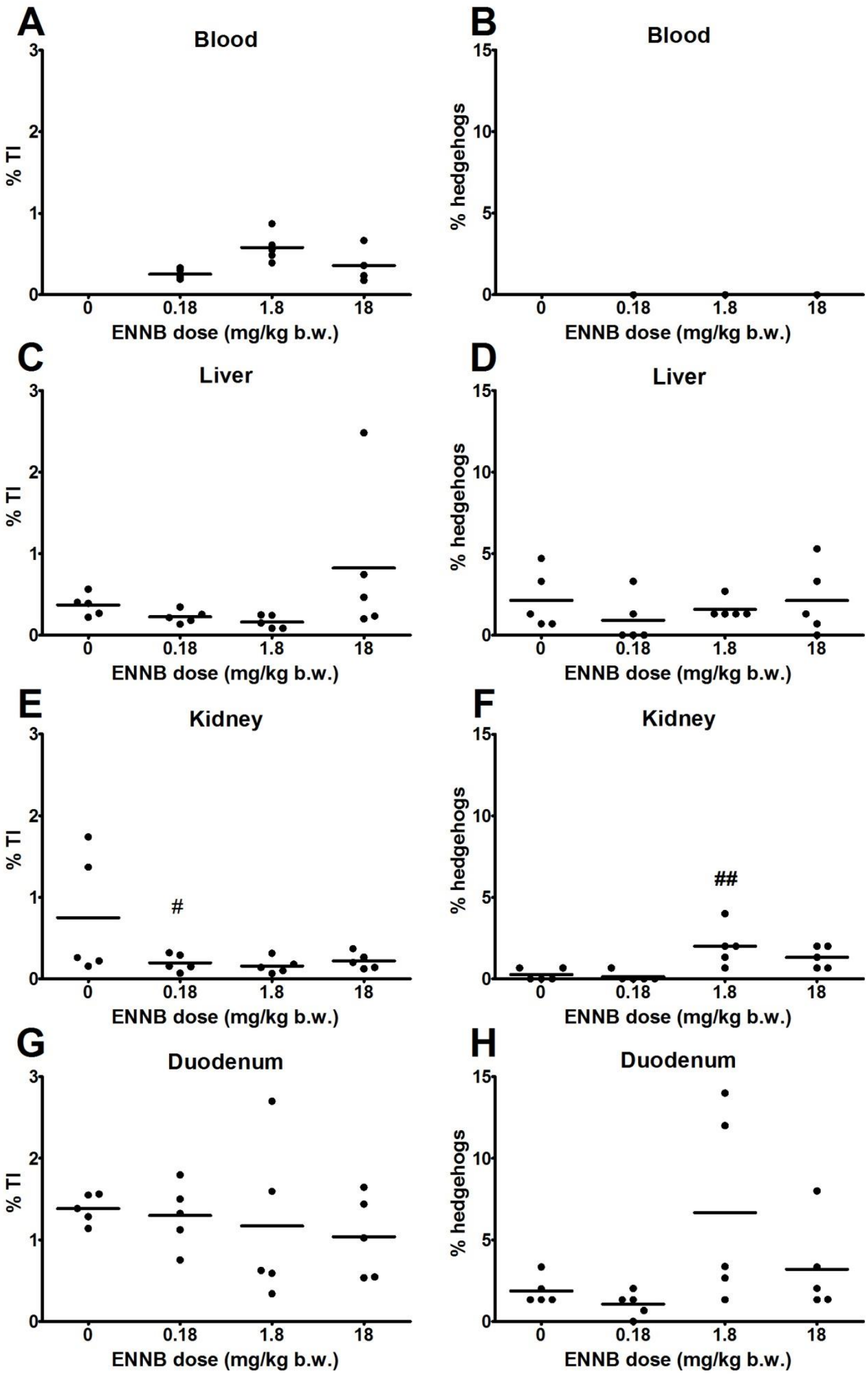

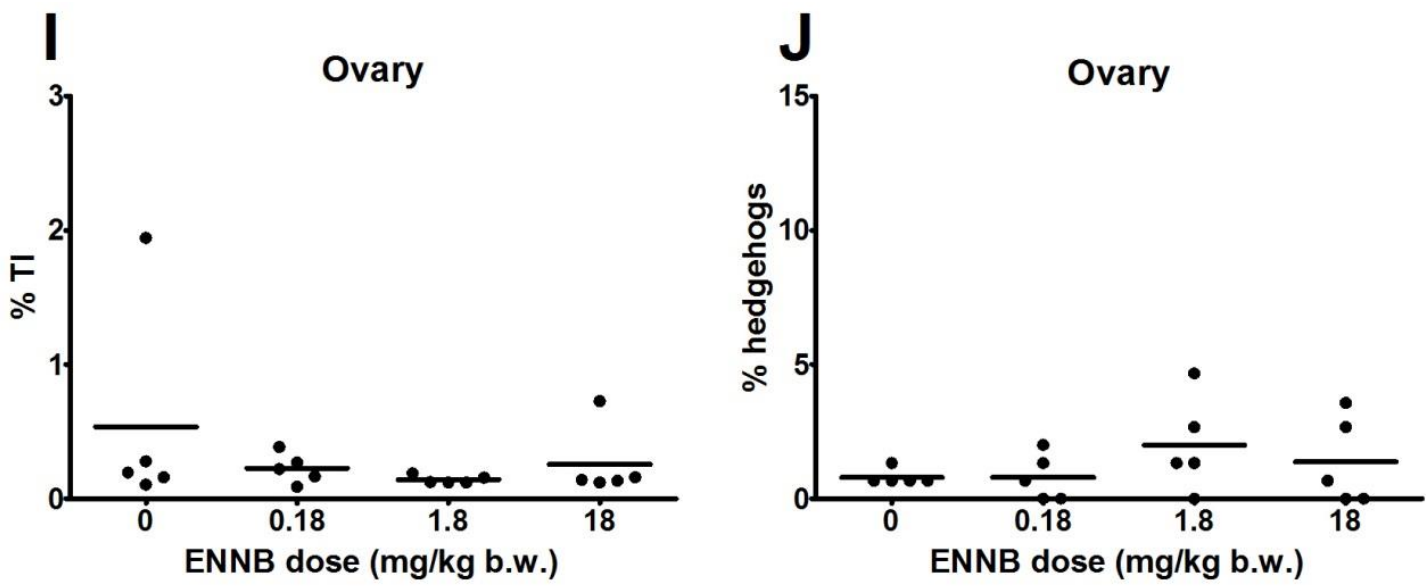

Figure 56: Alkaline Comet assay in different organs/tissues of female after repeated oral treatments with different doses of ENNB ( 0 - olive oil+DMSO6\%; $0.18,1.8$ and $18 \mathrm{mg} / \mathrm{kg}$ per day). In A, C, E, G and I dots represent the median of tail intensity obtained for each animal and lines represent the mean of tail intensity for each group. In $B, D, F, H$ and J dots represent the percentage of hedgehogs obtained for each mouse and lines are the mean for each group. \# $\mathrm{p}<0.05$, \#\# $\mathrm{p}<0.01$.

Comet assay results after ENNB treatment in male mice showed that the compound did not significantly increase the \%TI in any of the organs analyzed indicating that the compound, at the tested concentrations, was not able to induce DNA strand breaks in these organs. Similarly, in females and dams (see also paragraph 3.4.8) no significant increase of tail intensity was detected with respect to matched CTRL under any of the tested conditions. A significant decrease of tail length was observed at the intermediate dose in kidney cells of females, whose biological relevance is considered minor. In fact, there is no data suggesting a crosslinking effect of ENNB which might induce such a decrease of tail length. Furthermore, the protocol applied in the experiment was not the best suitable for the detection for this specific effect. The significant increase of \% hedgehogs detected in kidney at the intermediate dose only of female mice and at the higher dose in dams (see also paragraph 3.4.8) is not to be considered a strong indication of ENNB-related toxicity. In fact, the effect occurred at one dose only and was not massive, and the etiology of hedgehogs is uncertain (OECD TC $489^{1}$ ). Conversely, the significant increase of \% hedgehogs in ovary of dams appeared to be more substantial.

\section{Pig-a assay}

Pig-a assay results are summarized in Table 29 and Figure 57. No significant increase of mutant cells was observed after exposure to ENNB. However, notably, in the low and middle dose groups, samples appear more heterogeneous than in the other groups, and 5/6 samples in the low dose group and 4/5 samples in the middle dose group are substantially higher than the highest CTRL value. No alterations in the $\%$ of reticulocytes were observed after any ENNB dose.

\footnotetext{
${ }^{1}$ OECD, Test No. 489, 2014: In Vivo Mammalian Alkaline Comet Assay, OECD Publishing, Paris. 
Table 29: Pig-a assays in male mice after repeated oral treatments with different doses of ENNB (0 - olive oil+DMSO6\%; $0.18,1.8$ and $18 \mathrm{mg} / \mathrm{kg}$ per day).

\begin{tabular}{lccc}
\hline \multicolumn{1}{c}{ Treatment } & $\begin{array}{c}\text { \%RETs } \\
\text { mean } \pm \text { SE }\end{array}$ & $\begin{array}{c}\text { Mutant RBCs per 10 } \\
\text { Total RBCs } \\
\text { mean } \pm \text { SE }\end{array}$ & $\begin{array}{c}\text { Mutant RETs per 10 } \\
\text { Total RETs } \\
\text { mean } \pm \text { SE }\end{array}$ \\
\hline olive oil \pm DMSO6\% & $3.600 \pm 0.134$ & $0.150 \pm 0.043$ & $0.267 \pm 0.136$ \\
ENNB 0.18 & $3.350 \pm 0.214$ & $0.317 \pm 0.185$ & $1.950 \pm 0.718$ \\
ENNB 1.8 $^{\text {a }}$ & $3.560 \pm 0.413$ & $0.600 \pm 0.401$ & $2.060 \pm 0.703$ \\
ENNB 18 & $3.683 \pm 0.142$ & $0.167 \pm 0.049$ & $0.517 \pm 0.140$ \\
\hline
\end{tabular}

a mean and standard error are calculated on 5 mice only, excluding an outlier data that was one order of magnitude higher than the others, possibly as a consequence of a clonogenic effect.
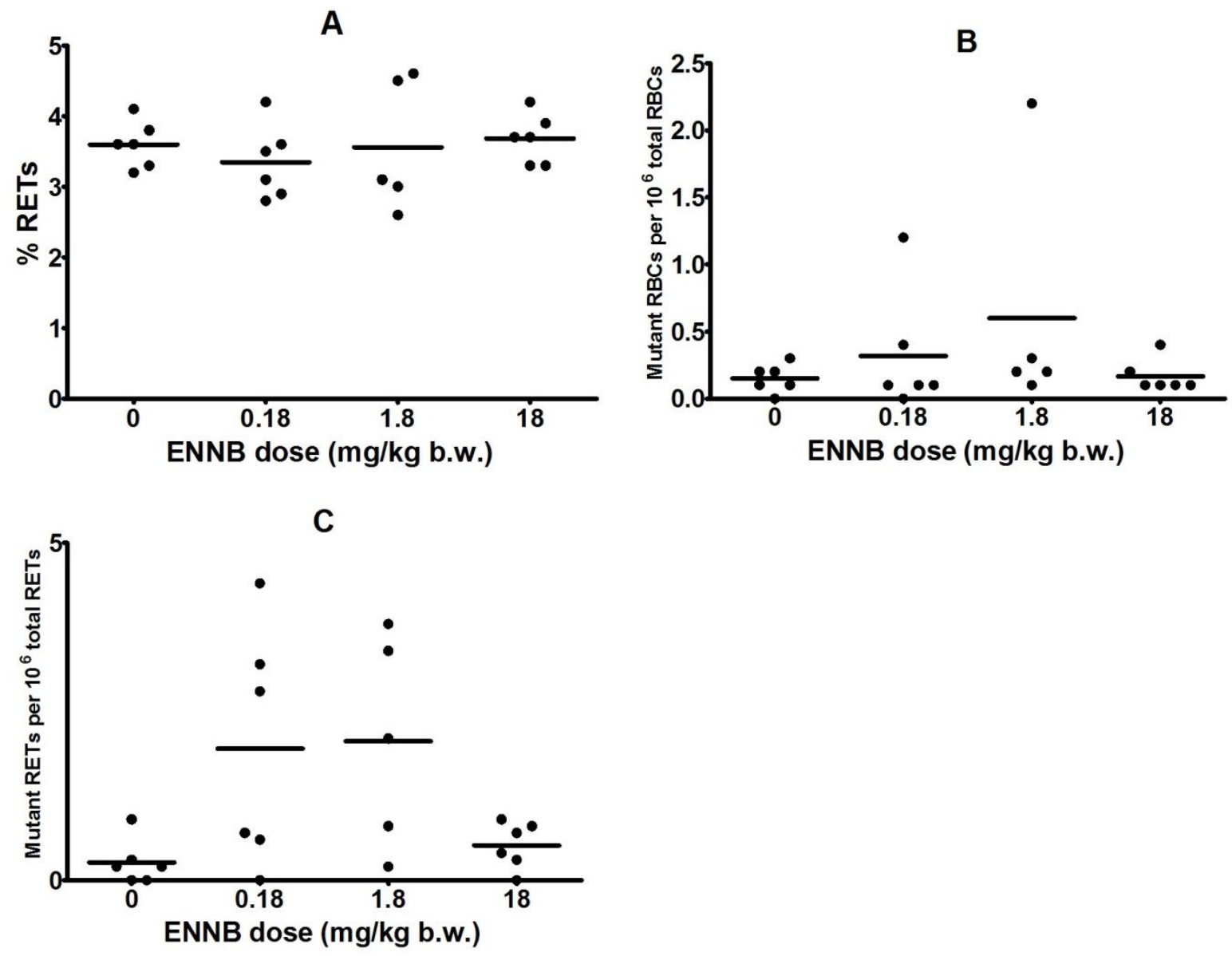

Figure 57: $\quad$ Pig-a assay in RBCs of male mice after repeated oral treatments with different doses of ENNB ( 0 - olive oil+DMSO6\%; $0.18,1.8$ and $18 \mathrm{mg} / \mathrm{kg}$ per day). Dots represent the percentage of RETs (A), the frequency of mutant RBCs (B) and the frequency of mutant RETs (C) obtained for each animal and lines represent the mean for each group. 


\section{Micronucleus test}

The MN test results collected are summarized in Table 30 and Figure 58. Data show that treatment with ENNB did not significantly increase the \%MN-RETs over the values of the CTRL group (olive oil+DMSO6\%). Moreover no changes in the percentage of reticulocytes were observed.

These results indicate that ENNB was not clastogenic or aneugenic in bone marrow precursors of red blood cells and was not cytotoxic in the same cells.

Table 30: $\quad$ MN assays in male mice after repeated oral treatments with different doses of ENNB (0 - olive oil+DMSO6\%; $0.18,1.8$ and $18 \mathrm{mg} / \mathrm{kg}$ per day).

\begin{tabular}{lcc}
\hline Treatment & \%RETs & \%MN-RETs \\
\hline olive oil+DMS06\% & $1.438 \pm 0.052$ & $0.130 \pm 0.018$ \\
ENNB0.18 & $1.547 \pm 0.183$ & $0.125 \pm 0.014$ \\
ENNB1.8 & $1.175 \pm 0.110$ & $0.138 \pm 0.014$ \\
ENNB18 & $1.785 \pm 0.160$ & $0.153 \pm 0.028$ \\
\hline
\end{tabular}
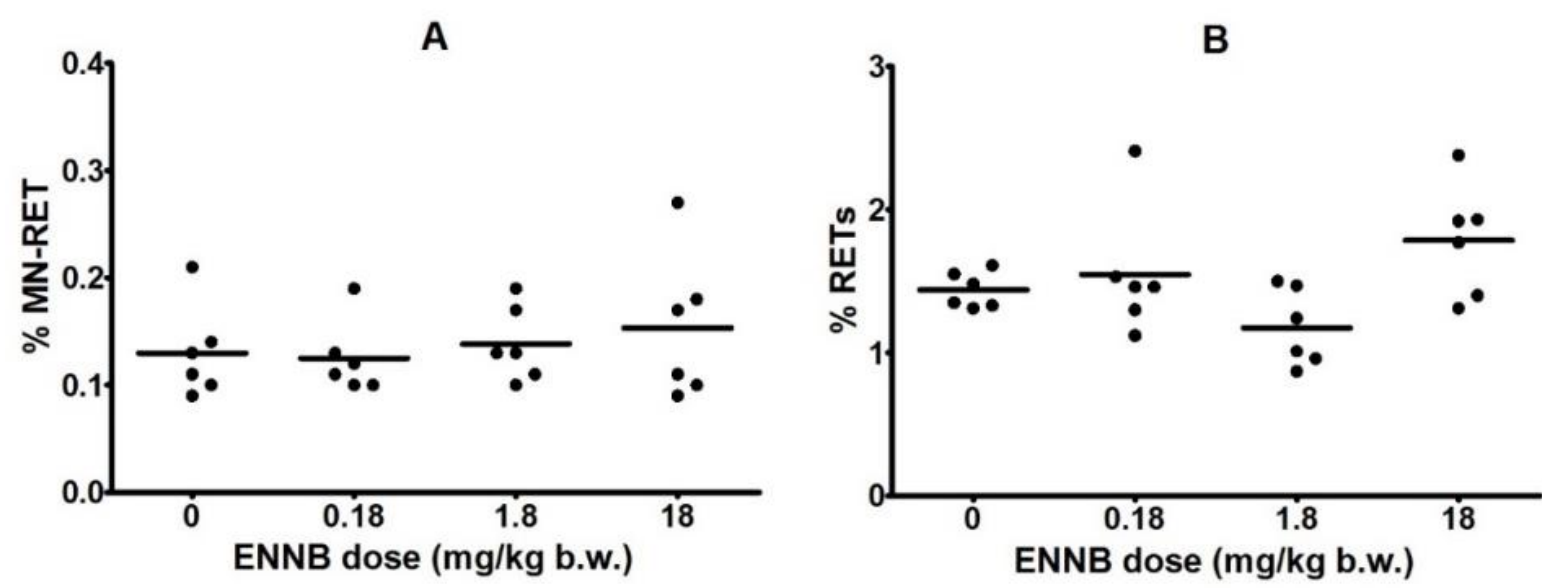

Figure 58: Micronucleus assay in RETs of male mice after repeated oral treatments with different doses of ENNB ( 0 - olive oil+DMSO6\%; $0.18,1.8$ and $18 \mathrm{mg} / \mathrm{kg}$ per day). Dots represent the percentage of micronucleated reticulocytes $(A)$ and percentage of RETs $(B)$ obtained for each animal and lines represent the mean for each group. 


\subsection{Reproduction/Developmental Toxicity Screening Test with BEA and ENNB}

\subsection{1. $\quad$ BEA - General toxicity}

\section{Body weight}

The body weight of dams during pre-mating, gestation and post-delivery periods was not significantly affected by BEA treatment at any dose level (Fig. 59).

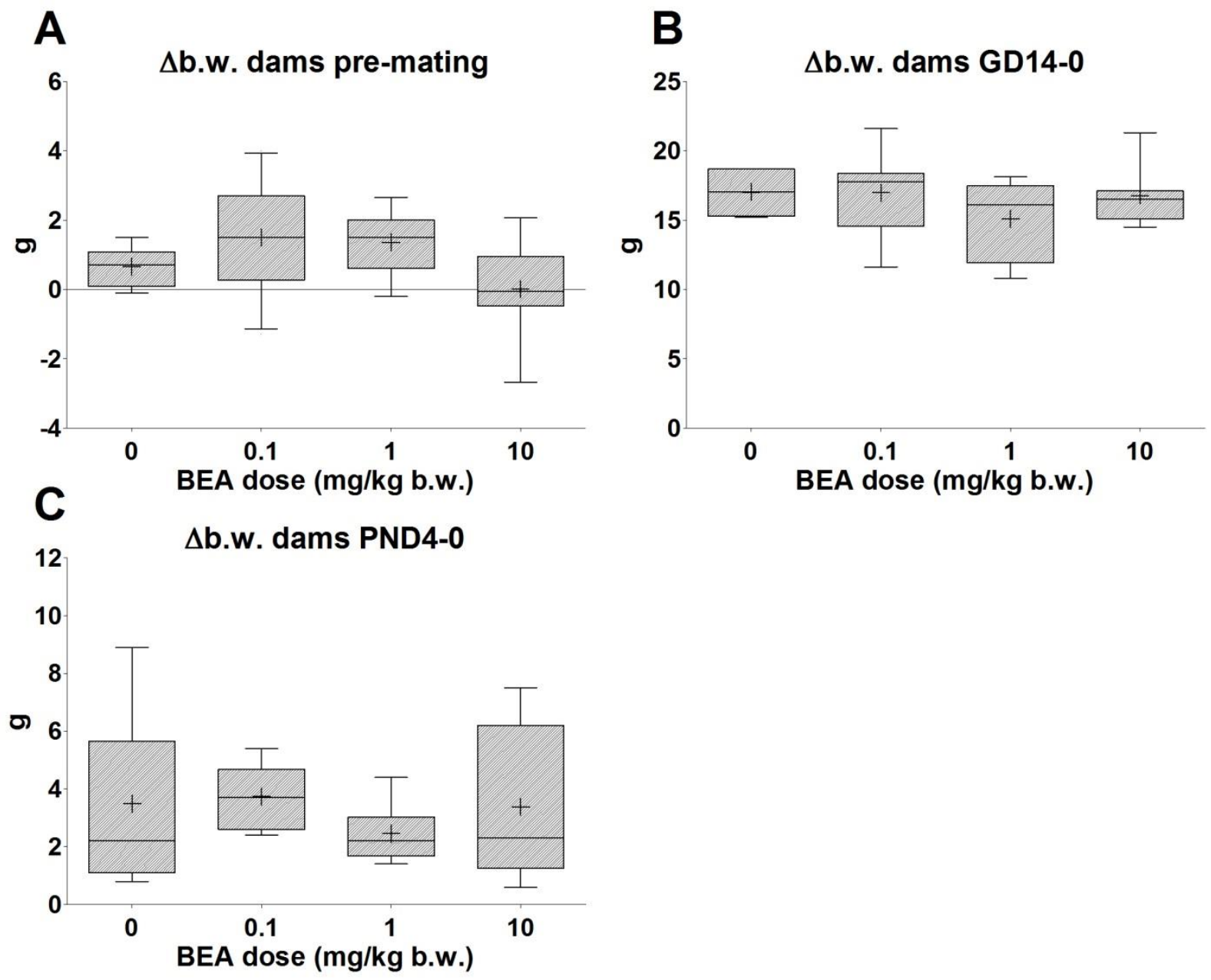

Figure 59: $\quad$ Mean differences in body weight during pre-mating time $(A)$ during gestation $(B)$ and post-partum (C) of dam mice orally treated with different doses of BEA ( 0 - olive oil; 0.1 , 1 and $10 \mathrm{mg} / \mathrm{kg}$ per day). Levels are reported as interquartile box plots, with whiskers delimiting the 10th and 90th percentiles. "+" indicates the mean value for each experimental group. 


\section{Food consumption}

BEA treatment did not affect the dams' food consumption (Fig. 60).

The time-to-pregnancy was not altered by the treatment (data not shown).

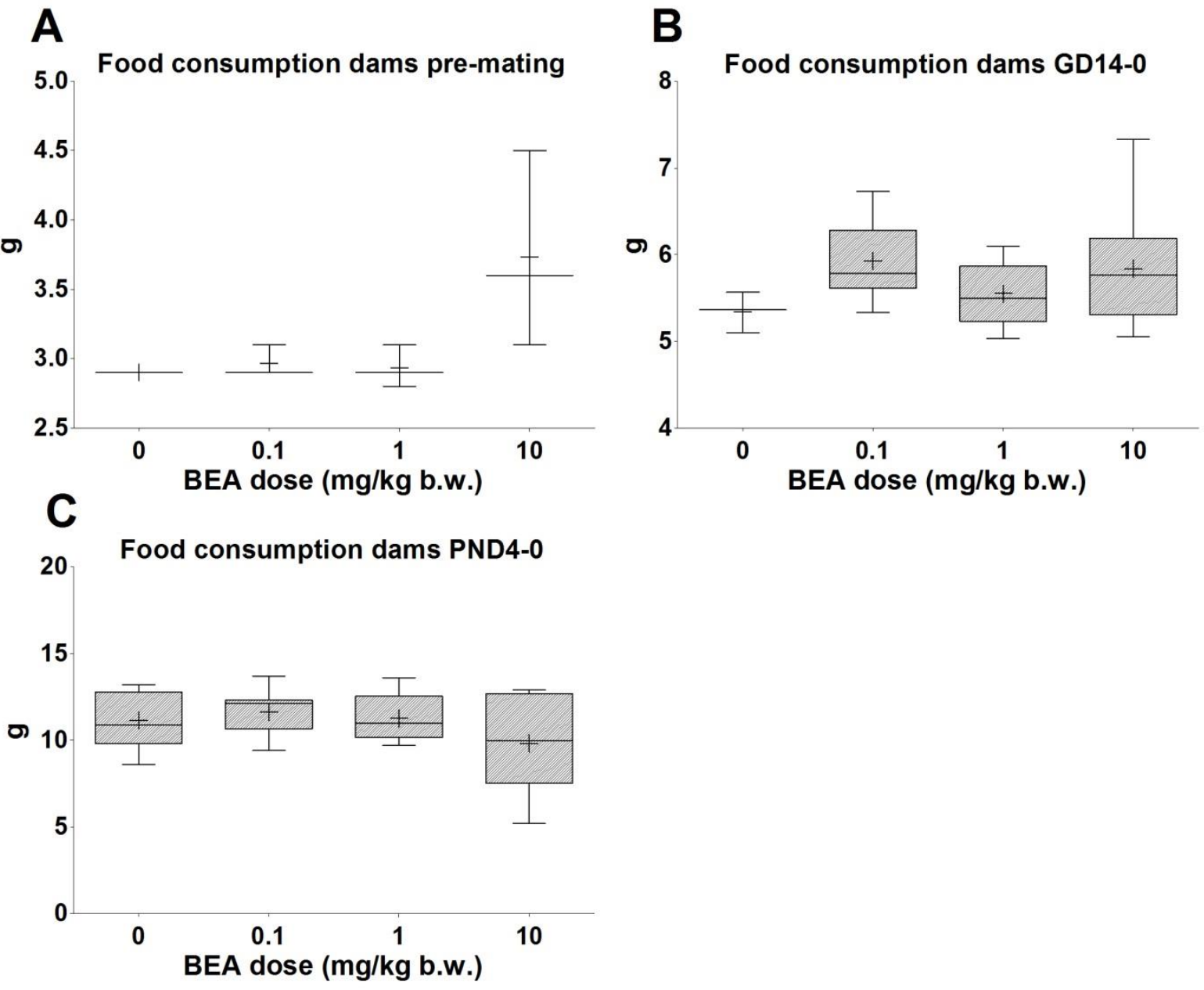

Figure 60: $\quad$ Mean food consumption: during pre-mating time $(A)$ during gestation $(B)$ and post partum (C) of dam mice treated orally with different doses of BEA ( 0 - olive oil; $0.1,1$ and $10 \mathrm{mg} / \mathrm{kg}$ per day). Levels are reported as interquartile box plots, with whiskers delimiting the 10th and 90th percentiles. "+" indicates the mean value for each experimental group.

\section{Pups}

The mean weight of pups at birth and at PND4 (Fig. 61A-B), as well as the litter weight at birth were not affected by treatment (Fig. 61C). Litter weight was significantly decreased at PND4 at BEA1: the correlation with the treatment is unclear (Fig. $61 \mathrm{D}$ ). 

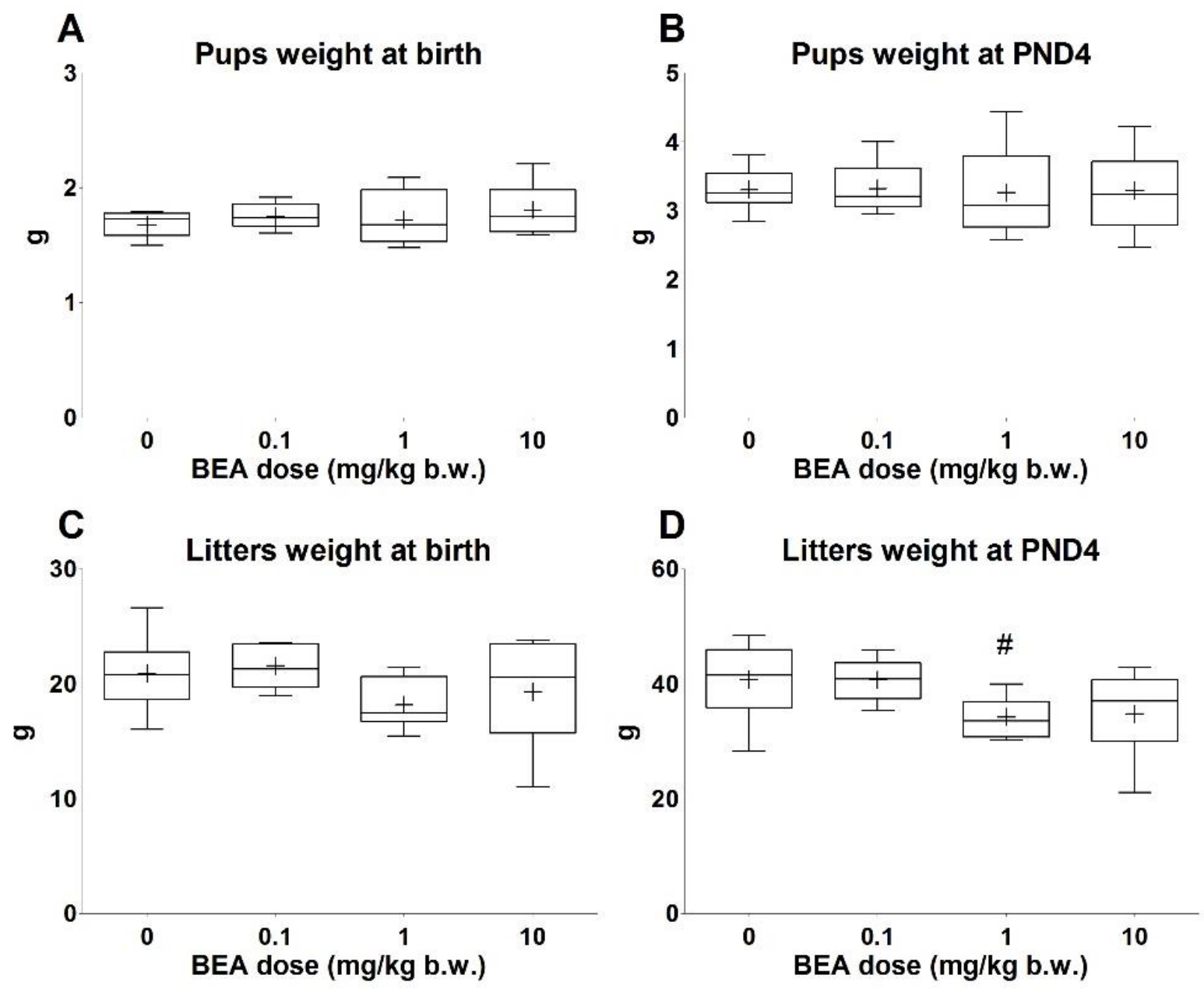

Figure 61: $\quad$ Mean pup weight at birth (A) and PND4 (B), litter weight at birth (C) and at PND4 (D) born from dams orally treated with different doses of BEA (0 - olive oil; $0.1,1$ and 10 $\mathrm{mg} / \mathrm{kg}$ per day). Levels are reported as interquartile box plots, with whiskers delimiting the 10 th and 90th percentiles. " + " indicates the mean value for each experimental group. \# $\mathrm{p}$ $<0.05$.

The sex ratio (males/females) was not significantly different in comparison to $C T R L$, as recorded at birth and at PND4 (Fig. 62). 

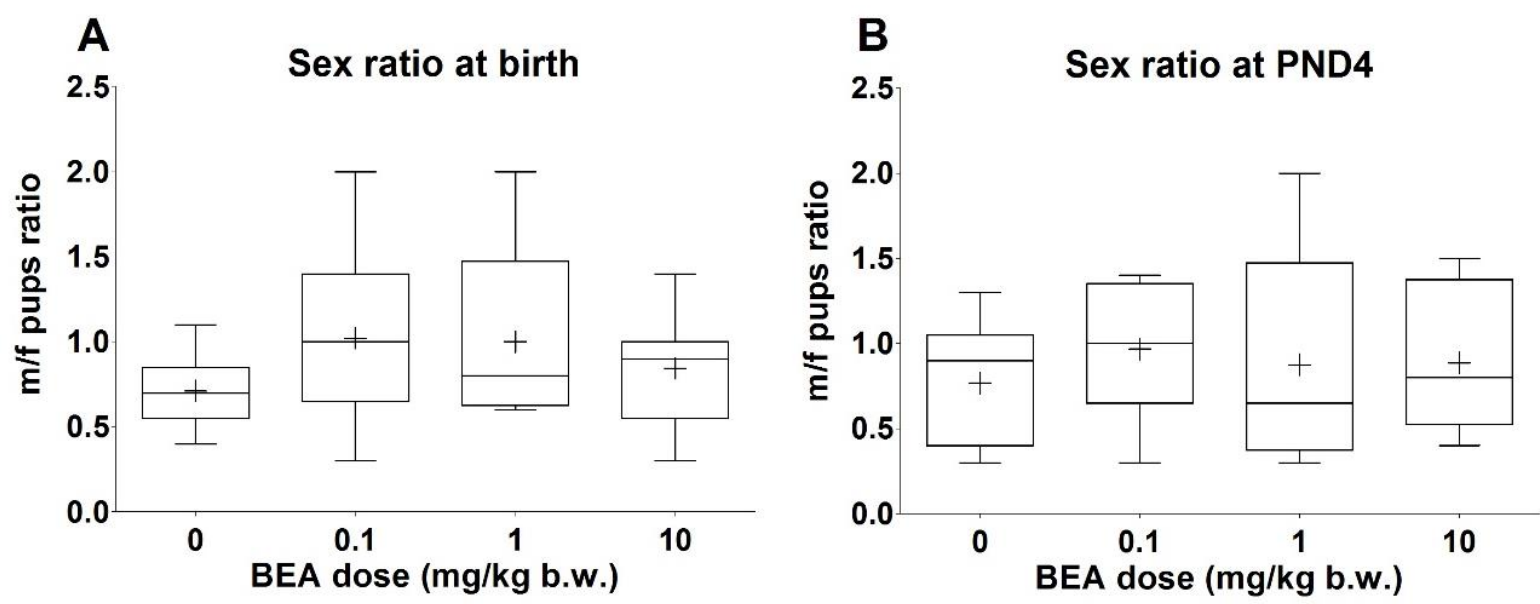

Figure 62: $\quad$ Mean pup sex ratio at birth (A) and at PND4 (B), born from dams orally treated with different doses of BEA ( 0 - olive oil; $0.1,1$ and $10 \mathrm{mg} / \mathrm{kg}$ per day). Levels are reported as interquartile box plots, with whiskers delimiting the 10th and 90th percentiles. "+" indicates the mean value for each experimental group

\section{Organ weight}

Dams absolute and relative organ weight are summarized in Table 31.

A significant increase in spleen absolute and relative weight was observed in BEA0.1-treated dams, the correlation with the treatment is unclear (Fig. 63C-D).

Thymus absolute and relative weight was significantly increased by BEA treatment at BEA1 and BEA10 dose levels (Fig. 63K-L).

Thyroid absolute and relative weight was significantly decreased at BEA0.1, the correlation with the treatment is unclear (Fig. 630-P).

Brain absolute and relative weight was significantly increased in dams treated with BEA0.1 and BEA1, the correlation with the treatment is unclear (Fig. 63Q-R).

Absolute and relative ovary weight was significantly increased in BEA10-treated dams compared to CTRLs (Fig. 64A-B). 
Table 31: Absolute and relative organ weights of dams orally treated with different doses of BEA (0 - olive oil+DMSO6\%; 0.1, 1 and $10 \mathrm{mg} / \mathrm{kg}$ per day)

\begin{tabular}{|c|c|c|c|c|c|}
\hline \multirow{2}{*}{ ORGAN } & & \multicolumn{4}{|c|}{ Dams } \\
\hline & & Olive oil & BEA0.1 & BEA1 & BEA10 \\
\hline & & mean $\pm S D$ & mean $\pm S D$ & mean $\pm S D$ & mean $\pm S D$ \\
\hline \multirow[t]{2}{*}{ LIVER } & $\begin{array}{l}N \text { of mice examined } \\
\text { Absolute weight }(\mathrm{g})\end{array}$ & $\begin{array}{c}8 \\
2.95 \pm 0.32\end{array}$ & $\begin{array}{c}8 \\
3.01 \pm 0.38\end{array}$ & $\begin{array}{c}8 \\
2.79 \pm 0.38\end{array}$ & $\begin{array}{c}10 \\
2.58 \pm 0.64\end{array}$ \\
\hline & Relative weight (x 100) & $7.89 \pm 1.04$ & $8.25 \pm 0.96$ & $7.78 \pm 1.11$ & $7.02 \pm 1.39$ \\
\hline \multirow[t]{3}{*}{ SPLEEN } & $N$ of mice examined & 8 & 9 & 9 & 9 \\
\hline & Absolute weight (g) & $0.16 \pm 0.03$ & $0.21 \pm 0.04 \#$ & $0.16 \pm 0.04$ & $0.17 \pm 0.05$ \\
\hline & Relative weight $(x$ 100) & $0.41 \pm 0.07$ & $0.58 \pm 0.09 \# \#$ & $0.43 \pm 0.11$ & $0.46 \pm 0.12$ \\
\hline \multirow[t]{3}{*}{ MLN } & $N$ of mice examined & 8 & 9 & 9 & 9 \\
\hline & Absolute weight (g) & $0.019 \pm 0.011$ & $0.008 \pm 0.004$ & $0.022 \pm 0.028$ & $0.131 \pm 0.214$ \\
\hline & Relative weight (x 100) & $0.047 \pm 0.029$ & $0.024 \pm 0.012$ & $0.058 \pm 0.075$ & $0.390 \pm 0.667$ \\
\hline \multirow[t]{3}{*}{ KIDNEYS } & $N$ of mice examined & 8 & 9 & 9 & 9 \\
\hline & Absolute weight (g) & $0.47 \pm 0.04$ & $0.49 \pm 0.04$ & $0.48 \pm 0.05$ & $0.49 \pm 0.04$ \\
\hline & Relative weight (x 100) & $1.24 \pm 0.10$ & $1.34 \pm 0.11$ & $1.35 \pm 0.13$ & $1.36 \pm 0.19$ \\
\hline \multirow[t]{3}{*}{ ADRENALS } & $N$ of mice examined & 8 & 8 & 9 & 9 \\
\hline & Absolute weight (g) & $0.012 \pm 0.005$ & $0.010 \pm 0.005$ & $0.013 \pm 0.004$ & $0.016 \pm 0.010$ \\
\hline & Relative weight (x 100) & $0.032 \pm 0.013$ & $0.028 \pm 0.014$ & $0.036 \pm 0.011$ & $0.043 \pm 0.030$ \\
\hline \multirow[t]{3}{*}{ THYMUS } & $N$ of mice examined & 7 & 9 & 9 & 9 \\
\hline & Absolute weight (g) & $0.01 \pm 0.01$ & $0.04 \pm 0.03$ & $0.05 \pm 0.02 \# \#$ & $0.06 \pm 0.03 \# \# \#$ \\
\hline & Relative weight (x 100) & $0.04 \pm 0.03$ & $0.12 \pm 0.09$ & $0.13 \pm 0.06 \# \#$ & $0.16 \pm 0.09 \# \#$ \\
\hline \multirow[t]{3}{*}{ HEART } & $N$ of mice examined & & & & \\
\hline & Absolute weight (g) & $0.23 \pm 0.03$ & $0.22 \pm 0.05$ & $0.25 \pm 0.04$ & $0.25 \pm 0.04$ \\
\hline & Relative weight (x 100) & $0.60 \pm 0.08$ & $0.60 \pm 0.12$ & $0.71 \pm 0.10$ & $0.68 \pm 0.09$ \\
\hline \multirow[t]{3}{*}{ THYROID } & $N$ of mice examined & 7 & 7 & 8 & 7 \\
\hline & Absolute weight (g) & $0.006 \pm 0.002$ & $0.003 \pm 0.001 \# \#$ & $0.004 \pm 0.001$ & $0.005 \pm 0.001$ \\
\hline & Relative weight (x 100) & $0.014 \pm 0.004$ & $0.008 \pm 0.008 \# \#$ & $0.011 \pm 0.003$ & $0.013 \pm 0.004$ \\
\hline \multirow[t]{3}{*}{ BRAIN } & $N$ of mice examined & 8 & 9 & 9 & 9 \\
\hline & Absolute weight (g) & $0.42 \pm 0.04$ & $0.43 \pm 0.12 \#$ & $0.48 \pm 0.02 \# \#$ & $0.44 \pm 0.03$ \\
\hline & Relative weight (x 100) & $1.11 \pm 0.12$ & $1.19 \pm 0.35 \#$ & $1.33 \pm 0.10 \# \#$ & $1.21 \pm 0.19$ \\
\hline \multirow[t]{3}{*}{ OVARY } & $N$ of mice examined & 8 & 9 & 9 & 9 \\
\hline & Absolute weight (g) & $0.02 \pm 0.01$ & $0.03 \pm 0.01$ & $0.02 \pm 0.01$ & $0.04 \pm 0.01 \# \#$ \\
\hline & Relative weight (x 100) & $0.05 \pm 0.01$ & $0.07 \pm 0.02$ & $0.07 \pm 0.01$ & $0.11 \pm 0.04 \# \# \#$ \\
\hline
\end{tabular}

\#\#\# p $<0.01 ; \#$ \# $<0.01 ; \# p<0.05$ 

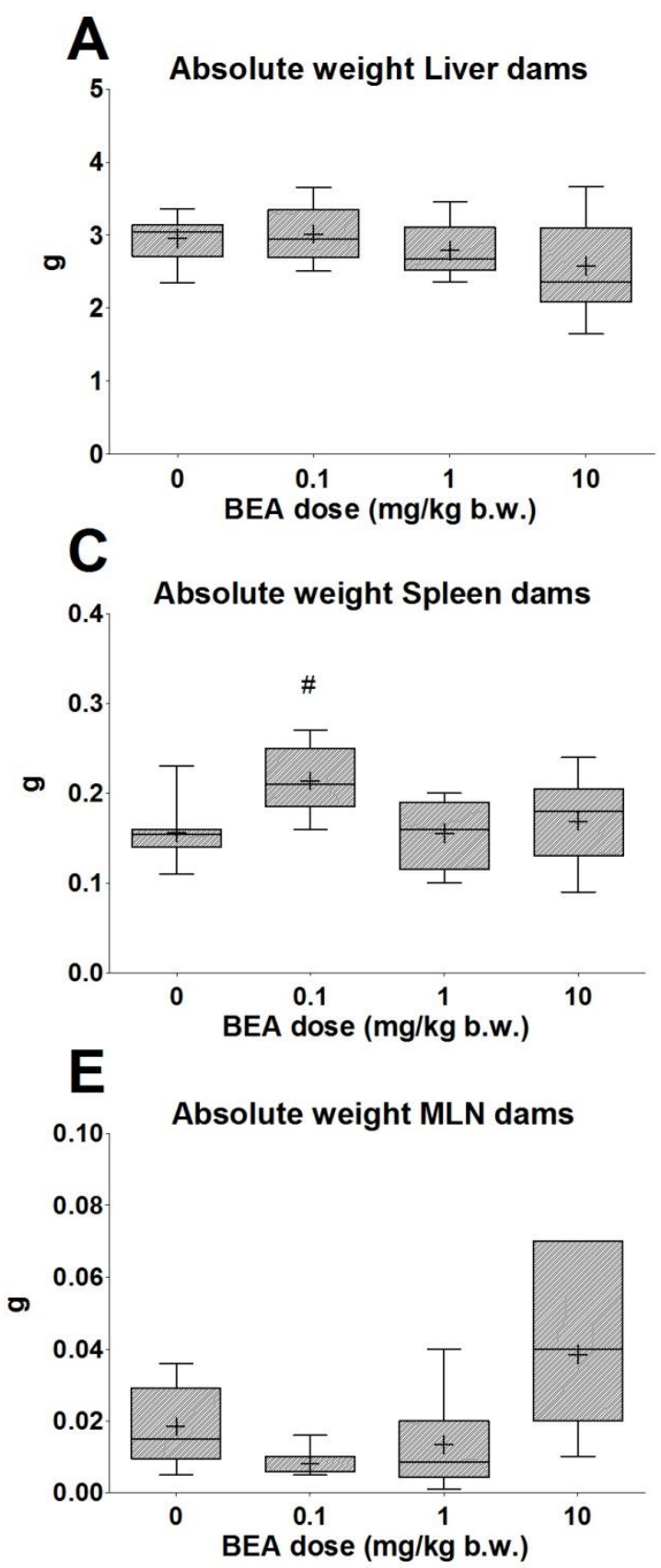

G

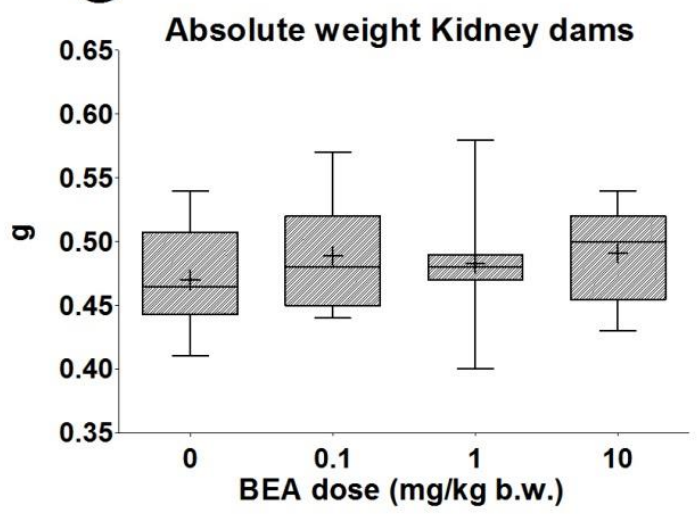

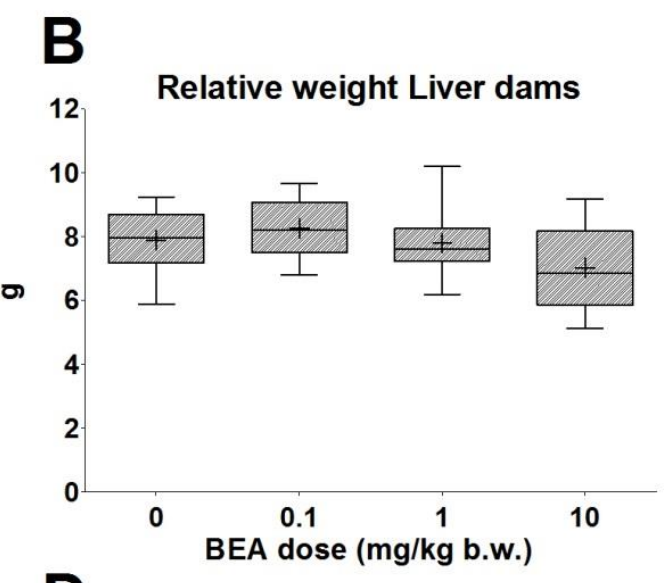

D
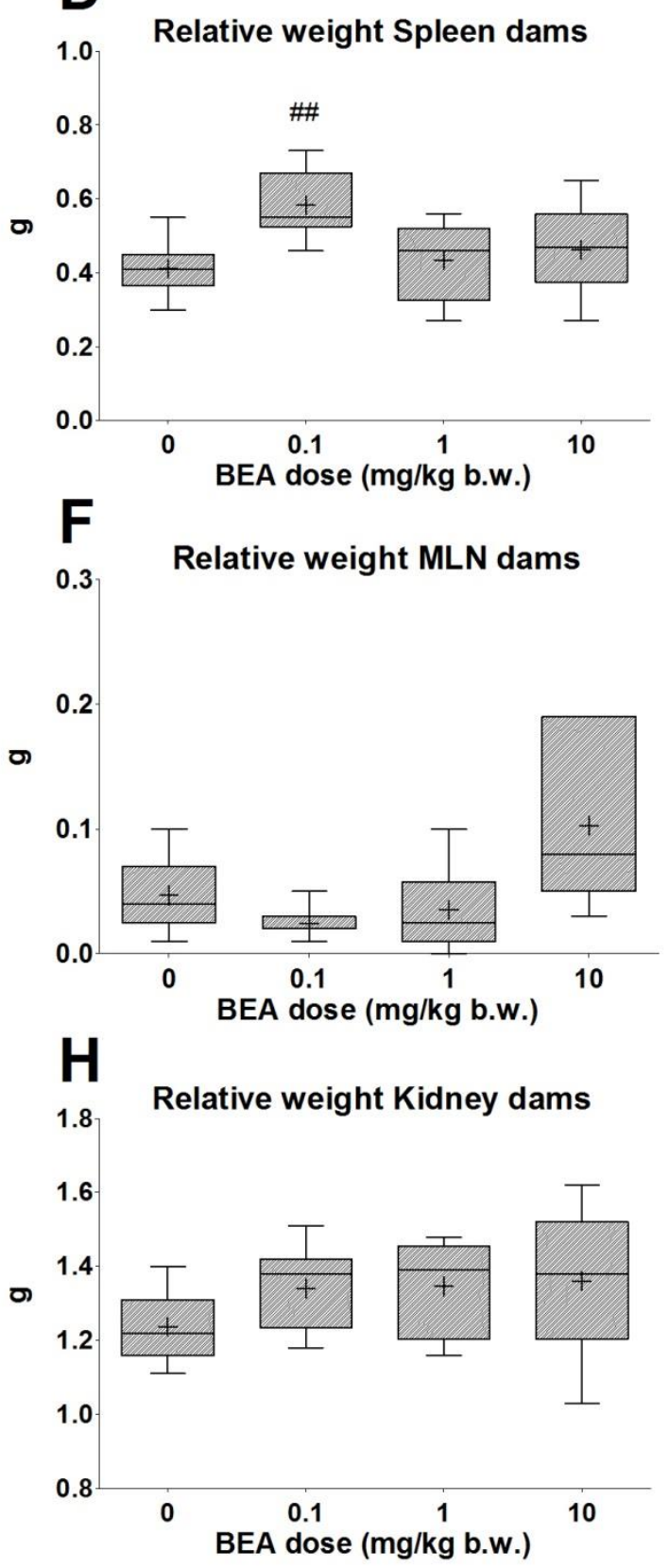

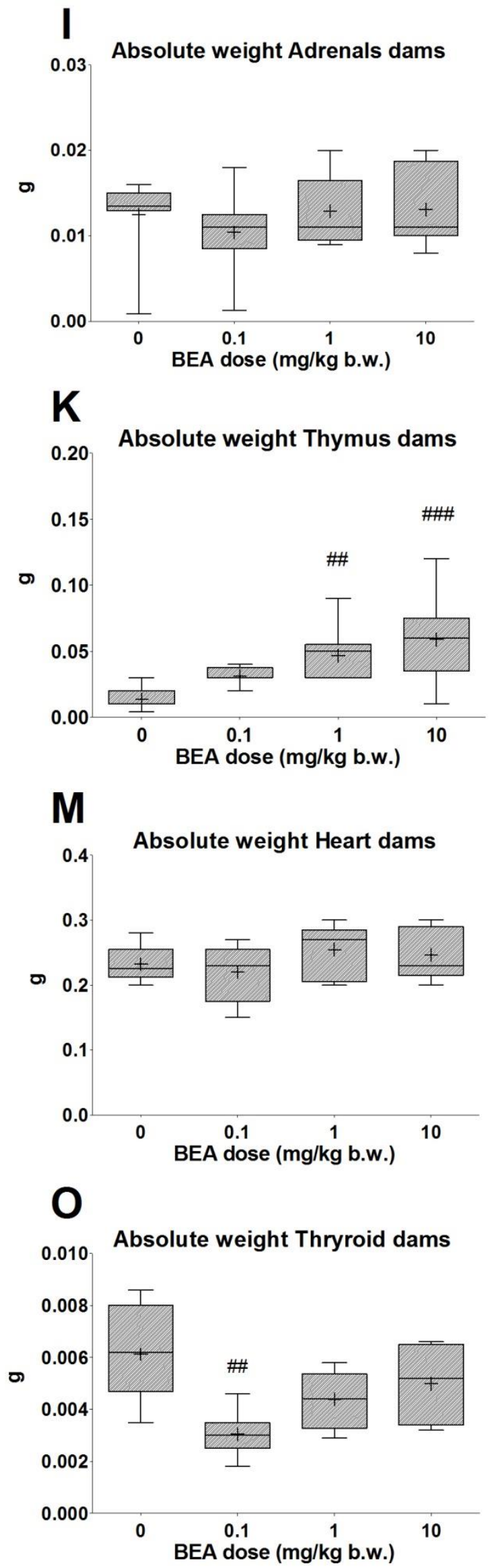
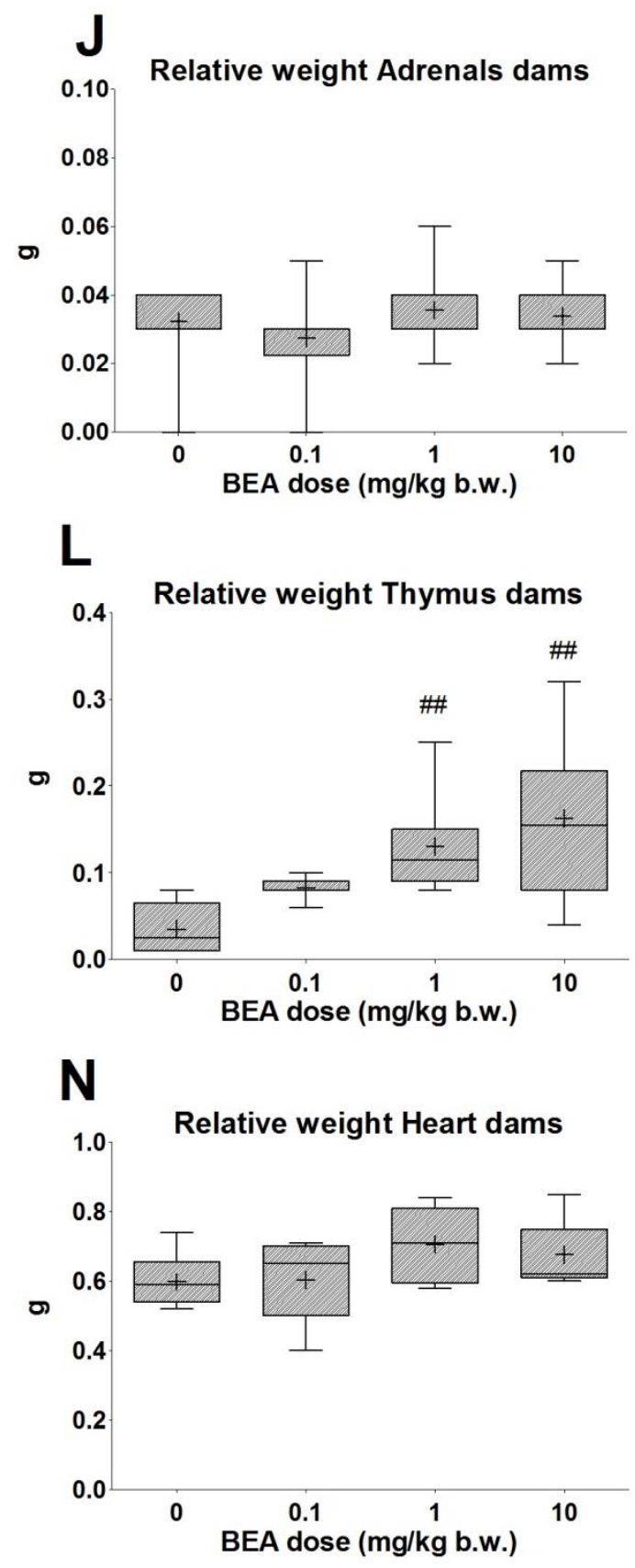

P Relative weight Thyroid dams

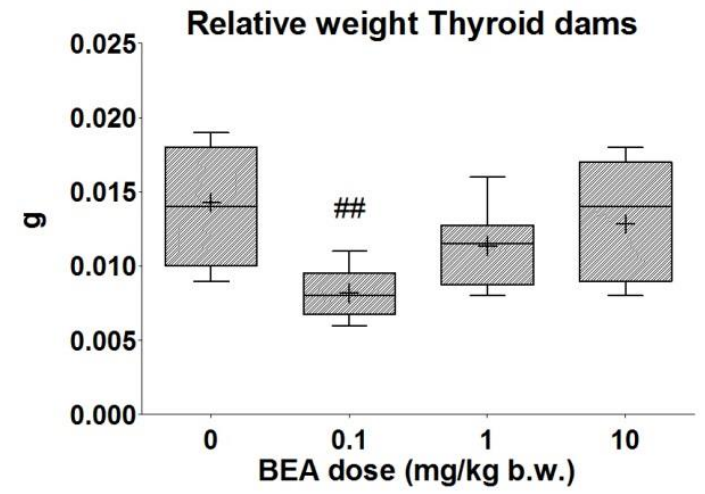



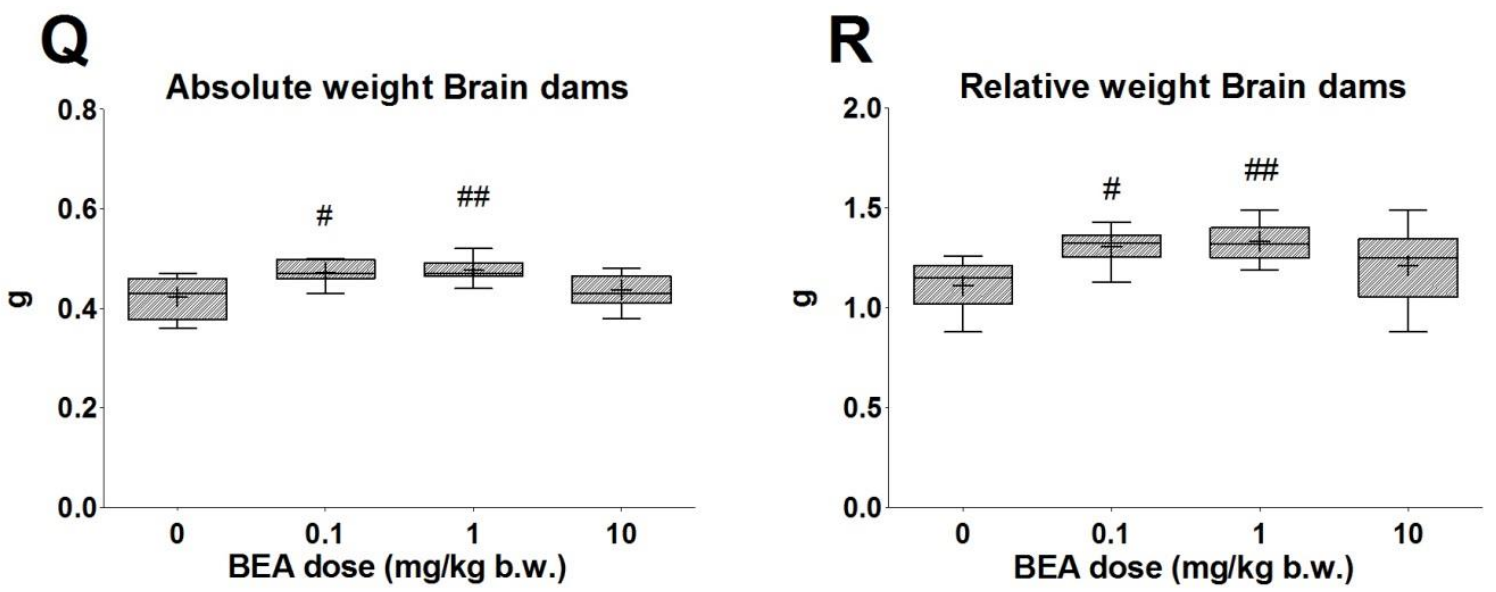

Figure 63: Absolute and relative organ weight of dams orally treated with different doses of BEA ( 0 - olive oil; $0.1,1$ and $10 \mathrm{mg} / \mathrm{kg}$ per day). Levels are reported as interquartile box plots, with whiskers delimiting the 10th and 90th percentiles. " + " indicates the mean value for each experimental group; \# $\mathrm{p}<0.05$; \# $\mathrm{p}<0.01$; \#\# $\mathrm{p}<0.001$.
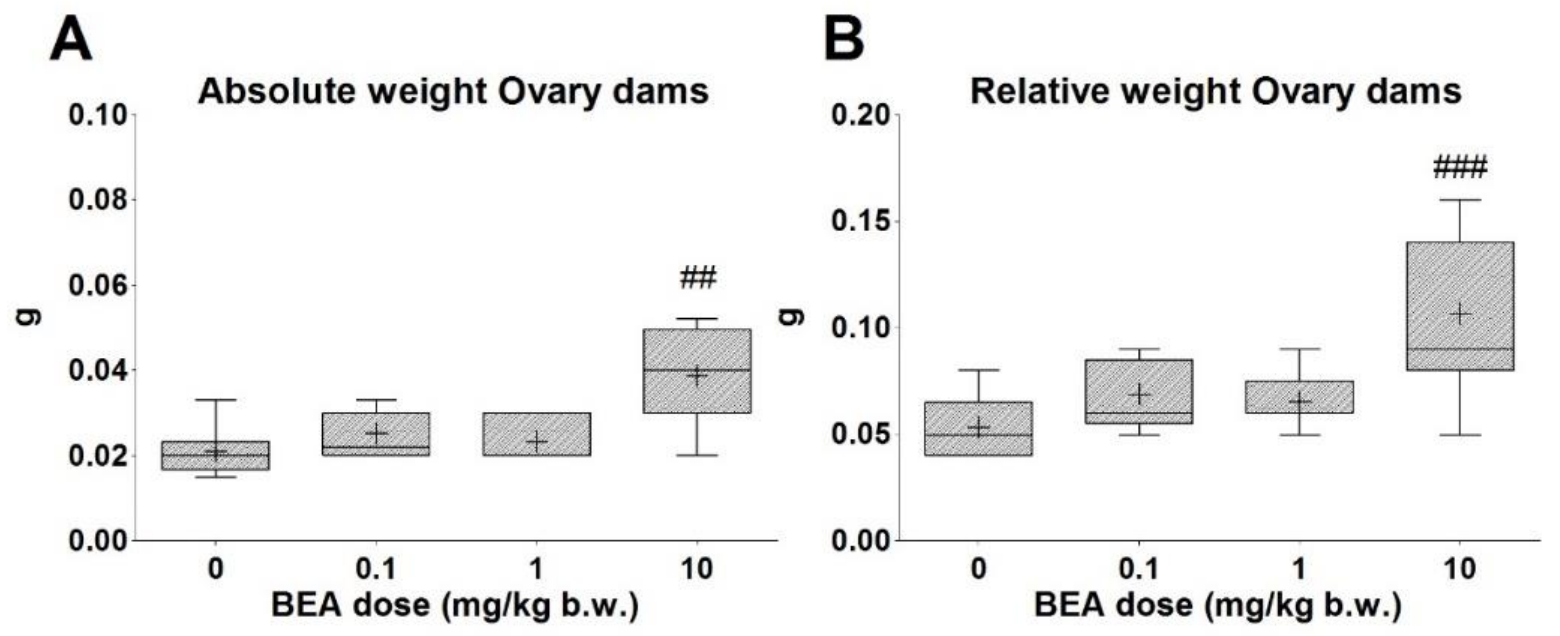

Figure 64: Absolute and relative ovary weights of dams orally treated with different doses of BEA ( 0 - olive oil; $0.1,1$ and $10 \mathrm{mg} / \mathrm{kg}$ per day). Levels are reported as interquartile box plots, with whiskers delimiting the 10th and 90th percentiles. " + " indicates the mean value for each experimental group. \#\# $\mathrm{p}<0.01 ; \# \#$ \# $<0.001$.

\subsubsection{BEA - Histopathological endpoints}

Histopathological endpoints in dams are in summarized Table 32. 
Table 32: Incidence of each histopathological endpoint analyzed in dam mice orally treated with different doses of BEA ( 0 - olive oil; $0.1,1$ and $10 \mathrm{mg} / \mathrm{kg}$ per day).

\begin{tabular}{|c|c|c|c|c|c|}
\hline \multirow{2}{*}{\multicolumn{2}{|c|}{ ORGAN/Effect }} & \multicolumn{4}{|c|}{ DAMS } \\
\hline & & Olive oil & BEA0.1 & BEA1 & BEA10 \\
\hline THYROID & $N$ of dams examined & 7 & 7 & 8 & 6 \\
\hline \multicolumn{6}{|l|}{ Colloid foaminess } \\
\hline & 0 & 2 & 5 & 5 & 1 \\
\hline & 1 & 4 & 2 & 3 & 3 \\
\hline & 2 & 1 & & & 2 \\
\hline & Total Finding Incidence & 5 & 2 & 3 & 5 \\
\hline \multicolumn{6}{|c|}{ Necrotic cells exfoliated into the colloid } \\
\hline & 0 & 6 & 5 & 7 & 5 \\
\hline & 1 & 1 & 2 & & 1 \\
\hline & 2 & & & 1 & \\
\hline & Total Finding Incidence & 1 & 2 & 1 & 1 \\
\hline \multicolumn{6}{|c|}{ Colloid reduction/absence in follicles } \\
\hline & 0 & 6 & 6 & 5 & 4 \\
\hline & 1 & 1 & & 1 & \\
\hline & 2 & & & 2 & 1 \\
\hline & 3 & & 1 & & 1 \\
\hline & Total Finding Incidence & 1 & 1 & 3 & 2 \\
\hline \multicolumn{6}{|l|}{ Pyknotic nuclei } \\
\hline & 0 & 6 & 7 & 2 & 1 \\
\hline & 1 & 1 & & 3 & 1 \\
\hline & 2 & & & 2 & 3 \\
\hline & 3 & & & 1 & 1 \\
\hline & Total Finding Incidence & 1 & 0 & 6 & 5 \\
\hline \multicolumn{6}{|l|}{ Follicles degeneration } \\
\hline & 0 & 7 & 7 & 3 & 2 \\
\hline & 1 & & & 3 & 1 \\
\hline & 2 & & & 2 & 3 \\
\hline & Total Finding Incidence & 0 & 0 & $5 \#$ & 4\# \\
\hline \multirow{5}{*}{$\begin{array}{l}\text { DUODENUM } \\
\text { Enterocytes vacuolation }\end{array}$} & $N$ of dams examined & 7 & 7 & 7 & 7 \\
\hline & 0 & 6 & 6 & 6 & 6 \\
\hline & 1 & 1 & & & 1 \\
\hline & 2 & & 1 & 1 & \\
\hline & Total Finding Incidence & 1 & 1 & 1 & 1 \\
\hline \multirow{4}{*}{$\begin{array}{l}\text { HEART } \\
\text { Chronic inflammation }\end{array}$} & Number examined & 7 & 7 & 7 & 7 \\
\hline & 0 & 7 & 7 & 6 & 7 \\
\hline & 1 & & & 1 & \\
\hline & Total Finding Incidence & 0 & 0 & 1 & 0 \\
\hline \multirow{7}{*}{$\begin{array}{l}\text { LIVER } \\
\text { Clear cell change }\end{array}$} & $N$ of dams examined & 7 & 7 & 7 & 7 \\
\hline & & & & & \\
\hline & 0 & 3 & 1 & 1 & 2 \\
\hline & 1 & 1 & 5 & 3 & 4 \\
\hline & 2 & 3 & 1 & 1 & 1 \\
\hline & 3 & & & 2 & \\
\hline & Total Finding Incidence & 4 & 6 & 6 & 5 \\
\hline \multicolumn{6}{|l|}{ Hepatocytic vacuolation } \\
\hline & 0 & 5 & 6 & 5 & 6 \\
\hline & 1 & & 1 & 1 & 1 \\
\hline & 2 & 2 & & 1 & \\
\hline & Total Finding Incidence & 2 & 1 & 2 & 1 \\
\hline \multicolumn{6}{|l|}{ Inflammatory cell foci } \\
\hline & 0 & 6 & 7 & 7 & 7 \\
\hline & 1 & 1 & & & \\
\hline & Total Finding Incidence & 1 & 0 & 0 & 0 \\
\hline
\end{tabular}

Grade 0: No change; Grade 1: Minimal; Grade 2: Mild; Grade 3: Moderate; Grade 4: Marked. 
Table 32 (continued): Incidence of each histopathological endpoint analyzed in dam mice orally treated with different doses of BEA ( 0 - olive oil; $0.1,1$ and $10 \mathrm{mg} / \mathrm{kg}$ per day).

DAMS

\begin{tabular}{|c|c|c|c|c|}
\hline ORGAN/Effect & Olive oil & BEA0.1 & BEA1 & BEA10 \\
\hline KIDNEYS & 7 & 7 & 7 & 7 \\
\hline Inflammatory cell focı & 6 & 7 & 7 & 7 \\
\hline 1 & 1 & & & \\
\hline Total Finding Incidence & 1 & 0 & 0 & 0 \\
\hline Tubular cell basophilia & & & & \\
\hline 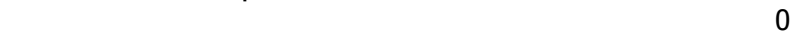 & 6 & 7 & 7 & 5 \\
\hline 2 & 1 & & & 2 \\
\hline Total Finding Incidence & 1 & 0 & 0 & 2 \\
\hline Tubular dilation & & & & \\
\hline 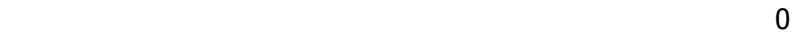 & 6 & 7 & 7 & 7 \\
\hline 2 & 1 & & & \\
\hline Total Finding Incidence & 1 & 0 & 0 & 0 \\
\hline SPLEEN & 7 & 7 & 7 & 7 \\
\hline 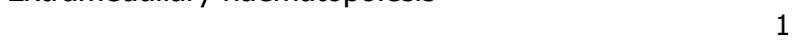 & & & 4 & 2 \\
\hline 2 & 7 & & & 1 \\
\hline 3 & & 7 & 3 & 4 \\
\hline Total Finding Incidence & 7 & 7\#\#\# & 7\#\#\# & 7\#\# \\
\hline$N$ of dams examined & 4 & 6 & 7 & 7 \\
\hline 0 & 3 & 4 & 3 & 4 \\
\hline 1 & 1 & 2 & 4 & 2 \\
\hline 2 & & & & 1 \\
\hline Total Finding Incidence & 1 & 2 & 4 & 3 \\
\hline$N$ of dams examined & 7 & 7 & 7 & 7 \\
\hline 1 & 3 & 3 & 3 & 5 \\
\hline 2 & 3 & 3 & 3 & 1 \\
\hline 3 & 1 & & 1 & \\
\hline Total Finding Incidence & 7 & 6 & 7 & 6 \\
\hline Subcapsular cell hyperplasia & & & & \\
\hline$e^{-1}$ & 6 & 7 & 7 & 6 \\
\hline 1 & 1 & & & \\
\hline 2 & & & & 1 \\
\hline Total Finding Incidence & 1 & 0 & 0 & 1 \\
\hline OVARY N of dams examined & 3 & 4 & 4 & 4 \\
\hline 0 & 2 & 2 & 2 & 1 \\
\hline 1 & 1 & 2 & & 1 \\
\hline 2 & & & 1 & 2 \\
\hline 3 & & & 1 & \\
\hline \begin{tabular}{ll} 
Total Finding Incidence \\
\cline { 2 - 2 }
\end{tabular} & 1 & 2 & 2 & 3 \\
\hline Degeneration follicles with degenerate oocyte & & & & \\
\hline 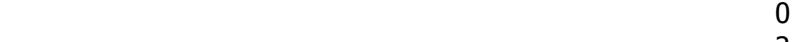 & 3 & 4 & 4 & 3 \\
\hline-2 & & & & 1 \\
\hline Total Finding Incidence & 0 & 0 & 0 & 1 \\
\hline
\end{tabular}

Grade 0: No change; Grade 1: Minimal; Grade 2: Mild; Grade 3: Moderate; Grade 4: Marked.

$\# \# \mathrm{p}<0.01 ; \# \#$ p $<0.001$ 
Table 32 (continued): Incidence of each histopathological endpoint analyzed in dam mice orally treated with different doses of BEA ( 0 - olive oil; $0.1,1$ and $10 \mathrm{mg} / \mathrm{kg}$ per day).

\begin{tabular}{lr|cccc}
\hline & \multicolumn{4}{c}{ DAMS } \\
\hline ORGAN/Effect & Nof dams examined & Olive oil & BEA0.1 & BEA1 & BEA10 \\
\hline BRAIN & & & 9 & 9 & 9 \\
Lymphatic infiltration & 0 & 9 & 8 & 3 & 5 \\
& 1 & & & 1 & \\
& 2 & & 1 & 1 & 4 \\
& 3 & & & 4 & 4 \\
\hline Spongiosis & Total Finding Incidence & 0 & 1 & 6 & \\
& & & & & \\
& & 6 & 8 & 8 & 7 \\
& 1 & & 1 & 1 & 1 \\
& & 3 & & 1 & 2 \\
\hline
\end{tabular}

Grade 0: No change; Grade 1: Minimal; Grade 2: Mild; Grade 3: Moderate; Grade 4: Marked.

Thyroid follicle degeneration was increased in BEA1 and BEA10 (Table 32). Histomorphometrical analysis showed decreased number of follicles and follicular density at BEA 10 (Fig. 65A-C).
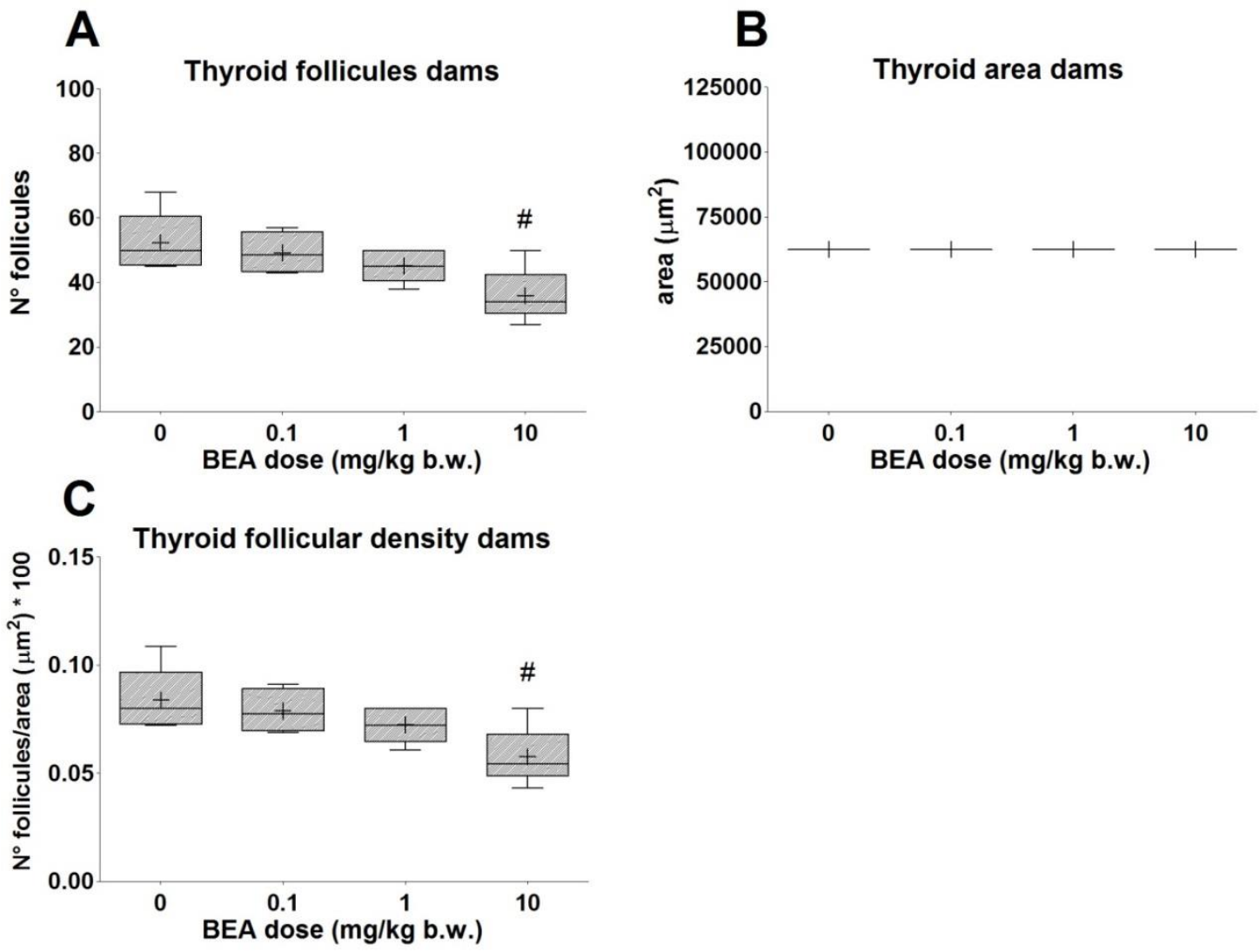

Figure 65: Histomorphometrical analysis of number of follicles $(A)$ and thyroid area (B); follicular density $(C)$ in dams orally treated with different doses of BEA ( 0 - olive oil; $0.1,1$ and 10 $\mathrm{mg} / \mathrm{kg}$ per day). Levels are reported as interquartile box plots, with whiskers delimiting the 10th and 90th percentiles. " + " indicates the mean value for each experimental group; \# $p$ $<0.05$

No histopathological alteration was observed in dam duodenum, heart and liver (Table 32). 
In spleen increased severity of EMH in all treated groups was present (Table 32).

Histomorphometrical analysis showed a significant increase in spleen red pulp area at BEA0.1 only, the correlation with the treatment is unclear (Fig. 66A).
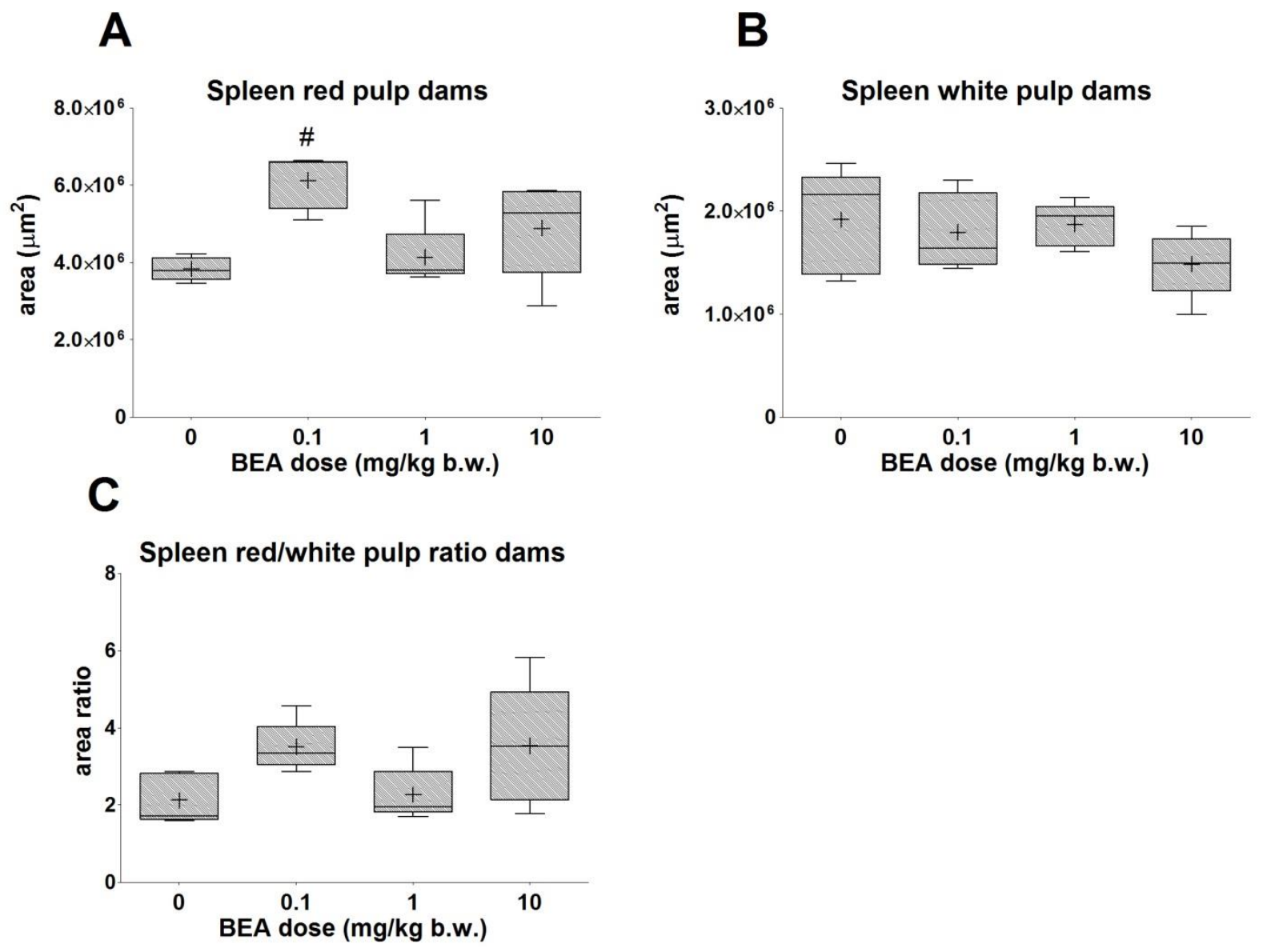

Figure 66: Histomorphometrical analysis of spleen red pulp area (A) and white pulp area (B); ratio of the red to white areas $(C)$ in dams orally treated with different doses of BEA ( 0 olive oil; $0.1,1$ and $10 \mathrm{mg} / \mathrm{kg}$ per day). Levels are reported as interquartile box plots, with whiskers delimiting the 10th and 90th percentiles. " + " indicates the mean value for each experimental group; \# $\mathrm{p}<0.05$

No histopathological and histmorphometrical effects were recorded in thymus (Table 32 and Fig. 67). 

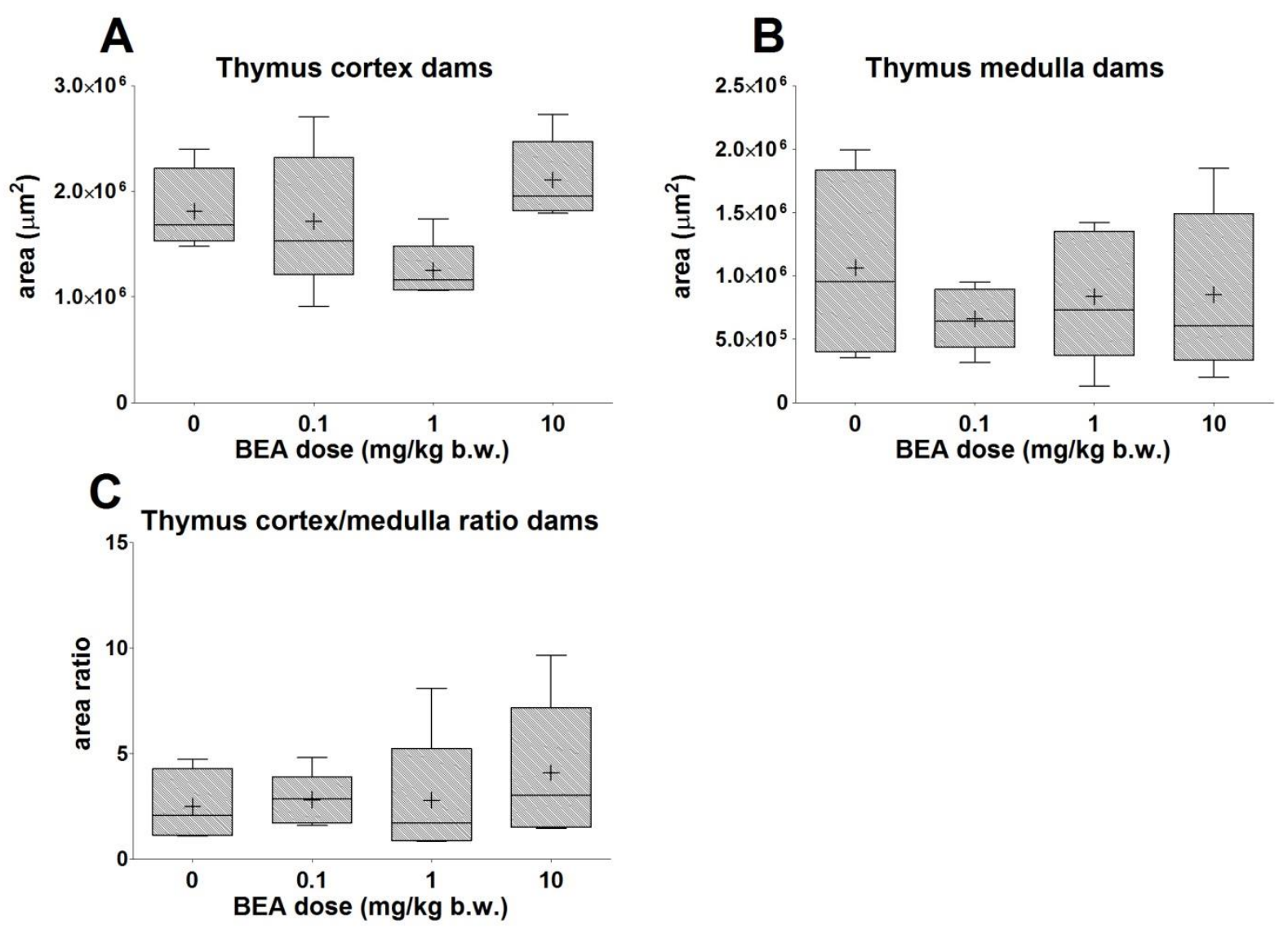

Figure 67: Histomorphometrical analysis of cortex (A) and medulla (B) thymus areas; ratio of the cortex to medulla areas (C) in dams orally treated with different doses of BEA ( 0 - olive oil; $0.1,1$ and $10 \mathrm{mg} / \mathrm{kg}$ per day). Levels are reported as interquartile box plots, with whiskers delimiting the 10th and 90th percentiles. "+" indicates the mean value for each experimental group

No effect was observed on histopathological and histomorphometrical endpoints in adrenals (Table 32 and Fig. 68). 

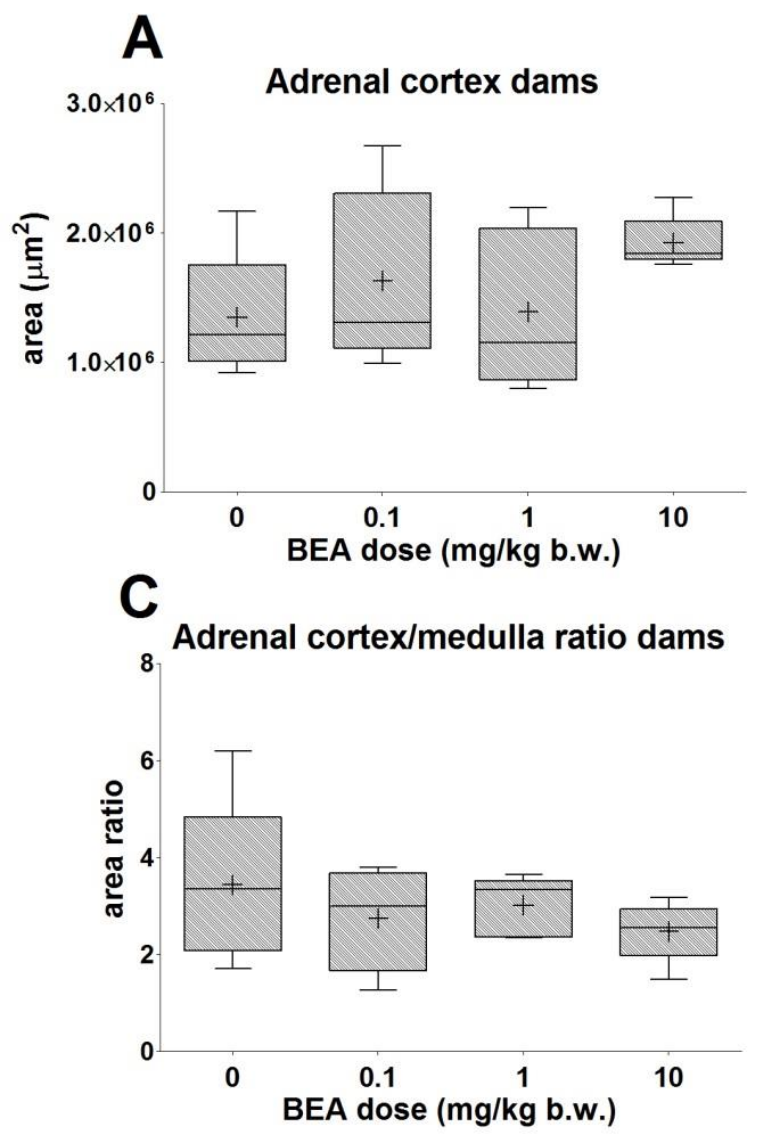

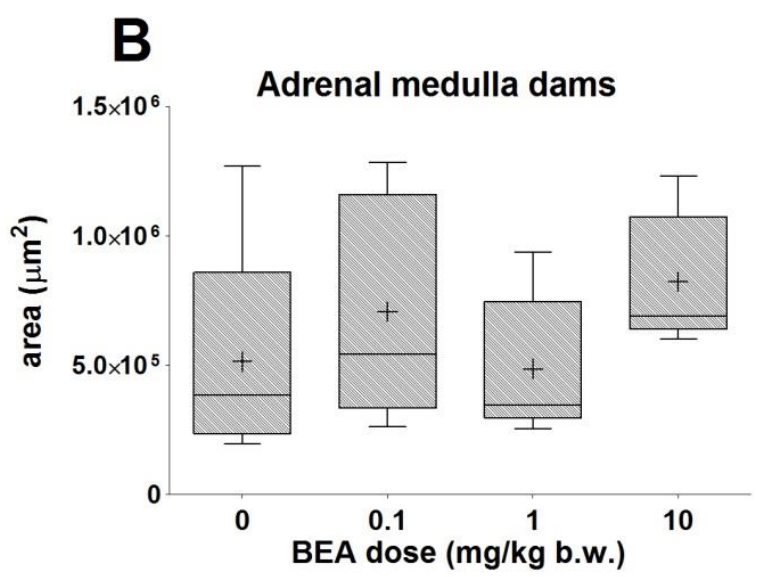

Figure 68: Histomorphometrical analysis of cortex (A) and medulla (B) adrenal areas; ratio of the cortex to medulla adrenal areas (C) of adrenals in dams orally treated with different doses of BEA ( 0 - olive oil; $0.1,1$ and $10 \mathrm{mg} / \mathrm{kg}$ per day). Levels are reported as interquartile box plots, with whiskers delimiting the 10th and 90th percentiles. " + " indicates the mean value for each experimental group

In brain, no histopathological effects were observed (Table 32).

The ovaries of dams were not affected by BEA treatment (Table 32 and Fig. 69). 
A

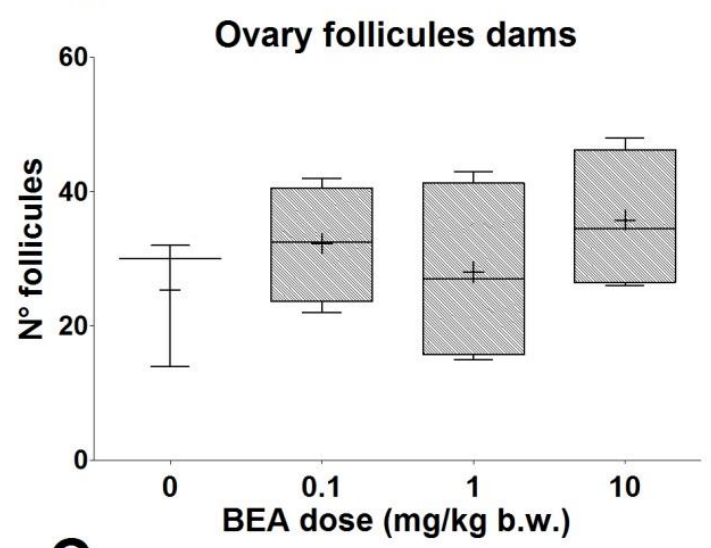

C

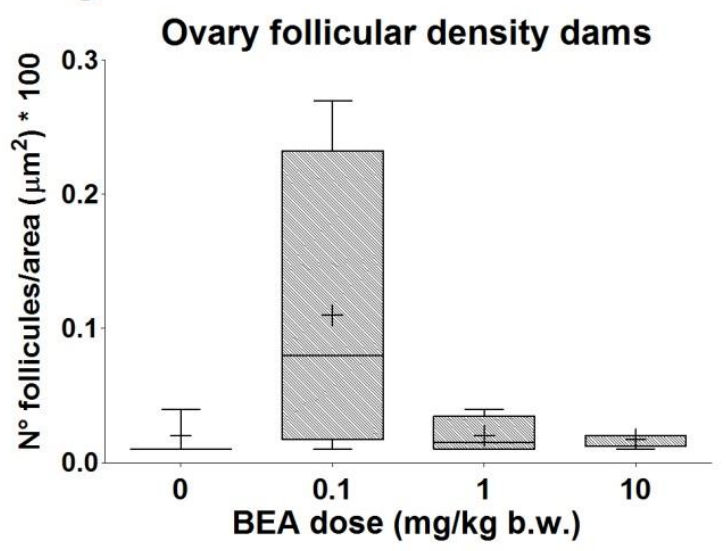

B

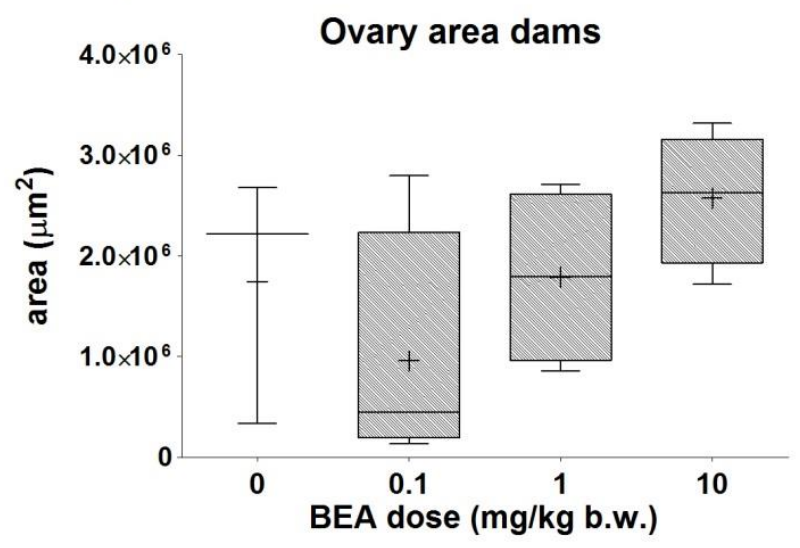

Figure 69: Histomorphometrical analysis of follicular number (A) and ovary area (B); follicular density $(C)$ of ovaries in dams mice treated orally with different doses of BEA ( 0 - olive oil; $0.1,1$ and $10 \mathrm{mg} / \mathrm{kg}$ per day). Levels are reported as interquartile box plots, with whiskers delimiting the 10th and 90th percentiles. " + " indicates the mean value for each experimental group

\subsubsection{BEA - Biomarker endpoints}

\section{Liver and kidney biomarkers}

No effect was recorded in dams for both liver and kidney biomarkers (Fig. 70) 
A

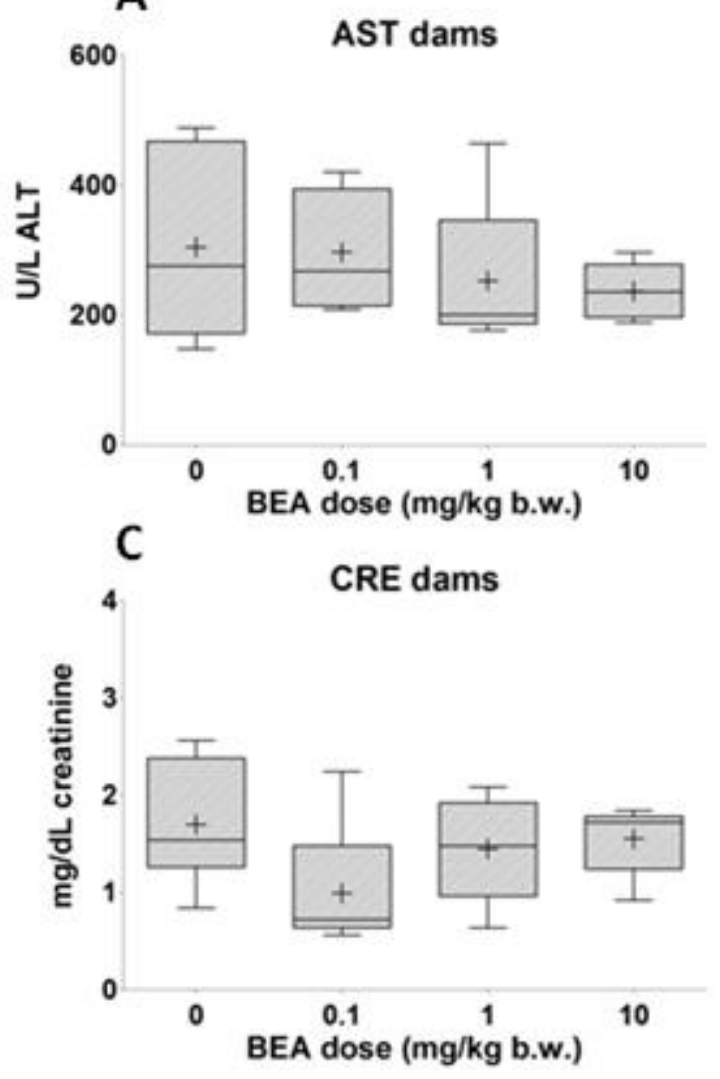

B
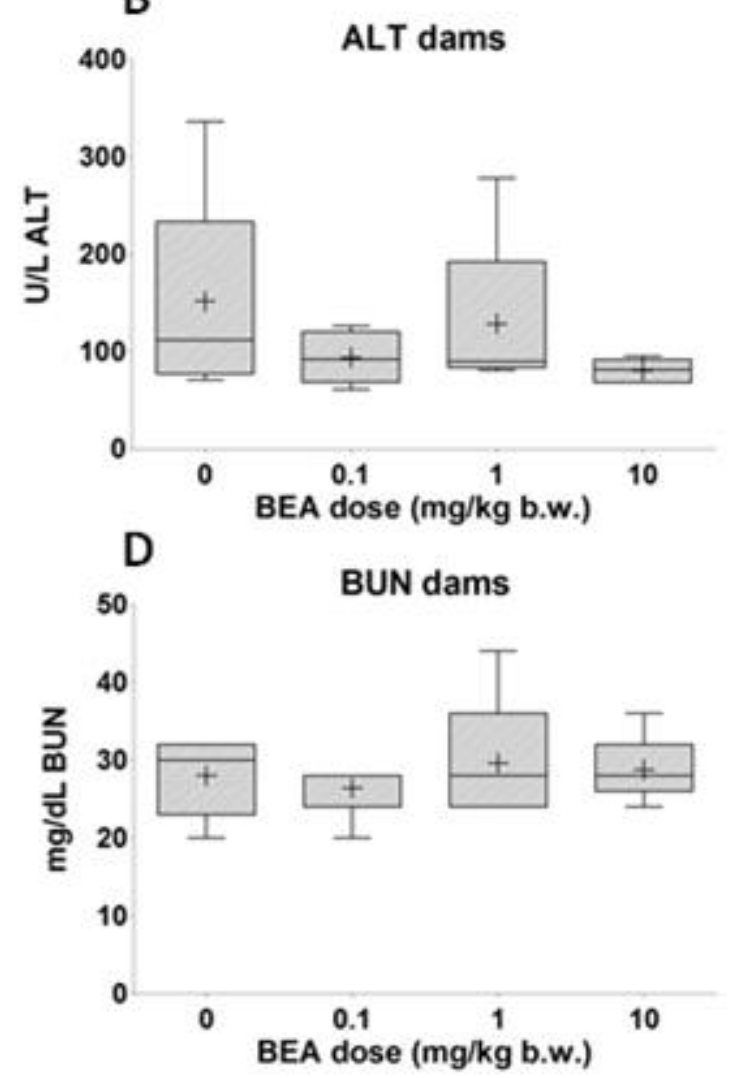

Figure 70: Serum levels of the liver biomarkers aspartate aminotransferase $(A)$ and alanine aminotransferase (B), and of the kidney biomarkers creatinine (C) and blood urea nitrogen (D) in dams mice orally treated with different doses of BEA ( 0 - olive oil; $0.1,1$ and 10 $\mathrm{mg} / \mathrm{kg}$ per day). Levels are reported as interquartile box plots, with whiskers delimiting the 10 th and 90th percentiles. " + " indicates the mean value for each experimental group; \# $p$ $<0.05$

\section{Hormone serum levels}

No effect was recorded in dams for both estradiol and testosterone serum levels (Fig. 71A-B)

No effect was observed on T4 serum levels (Fig. 71C) whereas TSH serum levels were significantly increased at BEA0.1, the correlation with the treatment is unclear (Fig. 71D). 
A
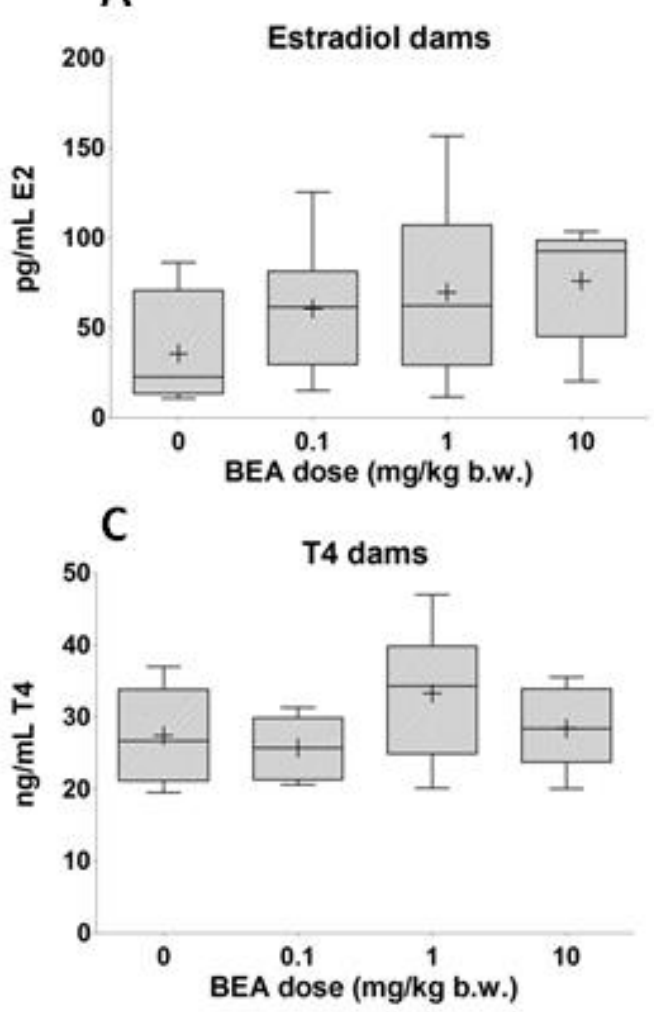

B
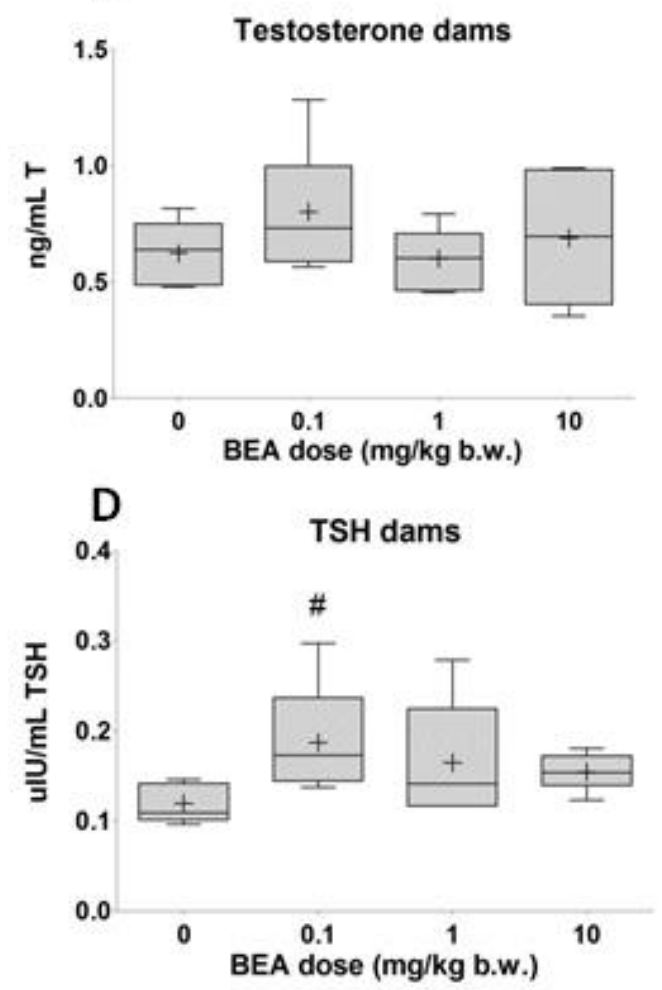

Figure 71: $\quad$ Serum levels of the hormones estradiol (A), testosterone (B), T4 (C) and TSH (D) in dams orally treated with different doses of BEA ( 0 - olive oil; $0.1,1$ and $10 \mathrm{mg} / \mathrm{kg}$ per day). Levels are reported as interquartile box plots, with whiskers delimiting the 10th and 90th percentiles. " + " indicates the mean value for each experimental group; \# $p<0.05$

\section{Oxidative stress markers in brain}

No significant alterations were observed in ROS and GSH brain levels in dams (Fig. 72).
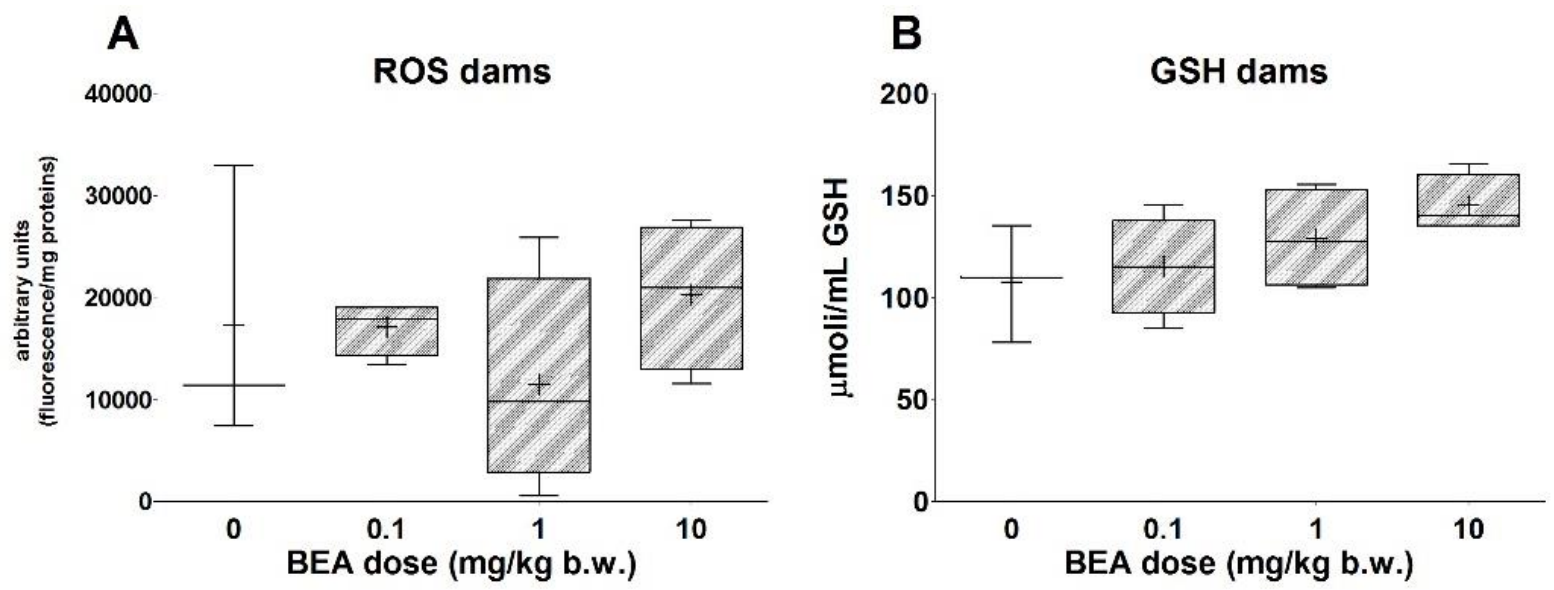

Figure 72: Brain levels of ROS (A) and GSH (B) in dams orally treated with different doses of BEA ( 0 - olive oil; $0.1,1$ and $10 \mathrm{mg} / \mathrm{kg}$ per day). Levels are reported as interquartile box plots, with whiskers delimiting the 10th and 90th percentiles. " + " indicates the mean value for each experimental group. 


\subsubsection{BEA - Genotoxicity endpoints}

\section{Comet assay}

Results obtained in dams are reported in Table 33 and Figure 73. No statistically significant difference was recorded in all organs, for all parameters analyzed. Data relative to the positive CTRL groups are those reported in the corresponding table relative to experiment with BEA repeated dose oral toxicity study.

Table 33: Alkaline Comet assay in different organs/tissues of dams after repeated oral treatments with different doses of BEA ( 0 - olive oil; $0.1,1$ and $10 \mathrm{mg} / \mathrm{kg}$ per day). For each experimental group the mean of median and standard error of the values obtained from each animal for tail length (Tail-L), tail intensity (\%TI) and Olive Tail Moment (OTM) are reported. The mean $\%$ hedgehogs for each group is shown.

\begin{tabular}{llllll}
\hline Tissue/organ & Treatment & $\begin{array}{c}\text { Tail-L } \\
\text { mean } \pm \text { SE }\end{array}$ & $\begin{array}{c}\text { \% TI } \\
\text { mean } \pm \text { SE }\end{array}$ & $\begin{array}{c}\text { OTM } \\
\text { mean } \pm \text { SE }\end{array}$ & $\begin{array}{c}\text { \% hedgehogs } \\
\text { mean } \pm \text { SE }\end{array}$ \\
\hline Blood & olive oil & $0.699 \pm 0.322$ & $0.301 \pm 0.085$ & $0.028 \pm 0.009$ & $0.562 \pm 0.415$ \\
& BEA0.1 & $2.908 \pm 1.241$ & $1.474 \pm 0.812$ & $0.174 \pm 0.097$ & $0.400 \pm 0.266$ \\
& BEA1 & $0.186 \pm 0.070$ & $0.143 \pm 0.016$ & $0.012 \pm 0.001$ & $0.267 \pm 0.267$ \\
& BEA10 & $0.349 \pm 0.177$ & $0.162 \pm 0.026$ & $0.014 \pm 0.002$ & $0.000 \pm 0.000$ \\
\hline Liver & olive oil & $4.628 \pm 2.433$ & $1.846 \pm 0.955$ & $0.267 \pm 0.156$ & $0.804 \pm 0.490$ \\
& BEA0.1 & $3.257 \pm 2.233$ & $0.854 \pm 0.614$ & $0.131 \pm 0.104$ & $2.666 \pm 1.264$ \\
& BEA1 & $0.606 \pm 0.086$ & $0.195 \pm 0.019$ & $0.019 \pm 0.002$ & $3.200 \pm 1.062$ \\
& BEA10 & $6.232 \pm 4.589$ & $0.809 \pm 0.387$ & $1.088 \pm 1.001$ & $0.532 \pm 0.326$ \\
\hline Kidney & olive oil & $2.838 \pm 1.153$ & $1.431 \pm 0.538$ & $0.158 \pm 0.068$ & $0.400 \pm 0.266$ \\
& BEA0.1 & $2.793 \pm 1.717$ & $1.550 \pm 0.983$ & $0.174 \pm 0.118$ & $0.134 \pm 0.134$ \\
& BEA1 & $0.233 \pm 0.065$ & $0.215 \pm 0.029$ & $0.020 \pm 0.003$ & $0.932 \pm 0.266$ \\
& BEA10 & $1.558 \pm 1.357$ & $1.493 \pm 1.268$ & $0.179 \pm 0.160$ & $0.800 \pm 0.249$ \\
\hline Duodenum & olive oil & $3.348 \pm 1.724$ & $3.381 \pm 2.307$ & $0.380 \pm 0.274$ & $2.400 \pm 0.956$ \\
& BEA0.1 & $1.605 \pm 0.739$ & $1.017 \pm 0.438$ & $0.110 \pm 0.051$ & $1.942 \pm 0.476$ \\
& BEA1 & $0.861 \pm 0.162$ & $0.785 \pm 0.192$ & $0.069 \pm 0.013$ & $3.870 \pm 1.162$ \\
& BEA10 & $6.264 \pm 3.817$ & $8.515 \pm 4.760$ & $1.200 \pm 0.783$ & $1.110 \pm 0.588$ \\
\hline Ovary & olive oil & $2.048 \pm 1.067$ & $1.682 \pm 0.840$ & $0.187 \pm 0.108$ & $0.666 \pm 0.298$ \\
& BEA0.1 & $0.908 \pm 0.439$ & $1.069 \pm 0.746$ & $0.094 \pm 0.063$ & $0.934 \pm 0.499$ \\
& BEA1 & $0.559 \pm 0.190$ & $0.328 \pm 0.085$ & $0.027 \pm 0.007$ & $2.133 \pm 1.555$ \\
& BEA10 & $0.605 \pm 0.291$ & $0.528 \pm 0.246$ & $0.043 \pm 0.020$ & $1.602 \pm 0.777$ \\
\hline
\end{tabular}



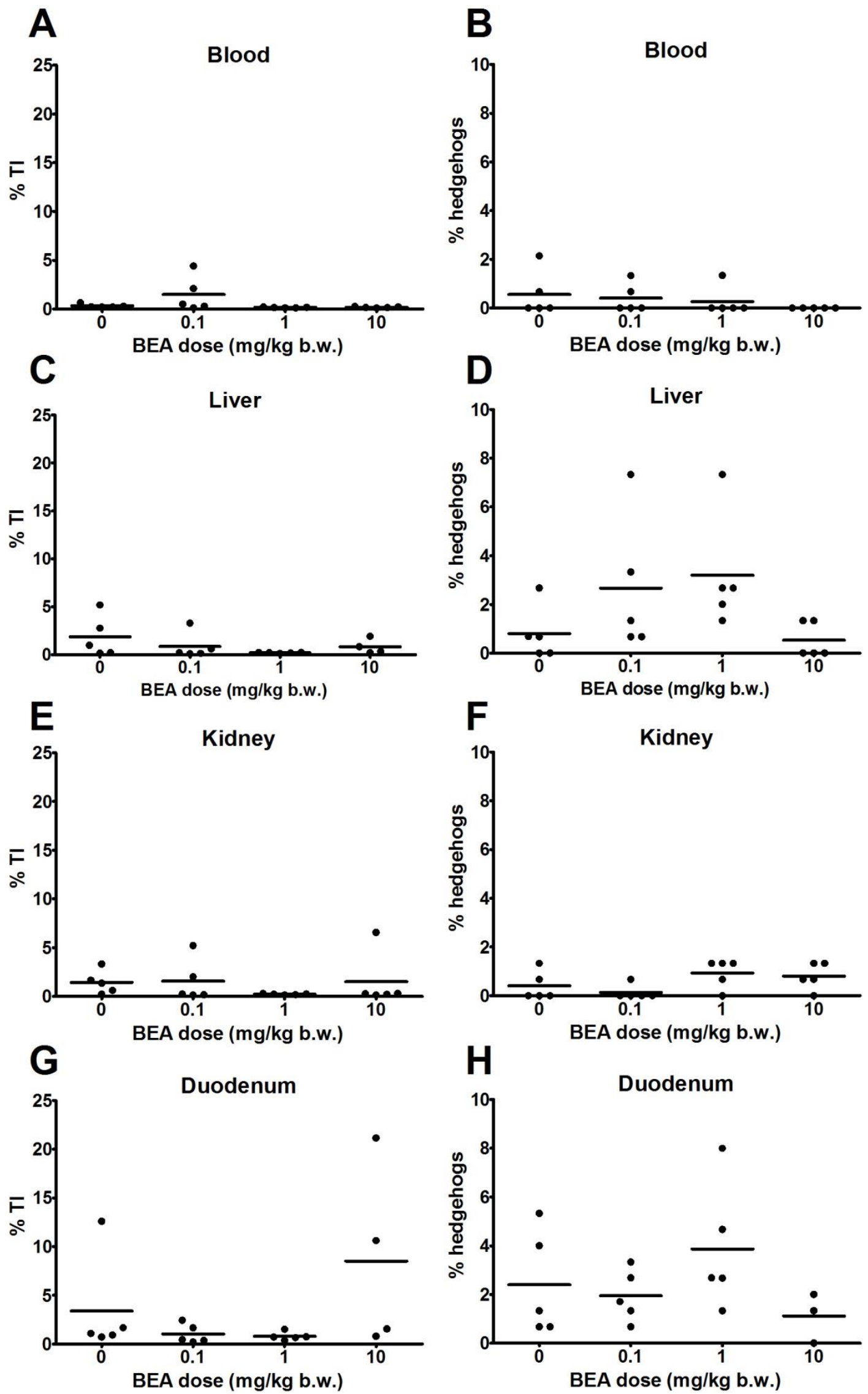

The present document has been produced and adopted by the bodies identified above as authors. In accordance with Article 36 of Regulation (EC) No $178 / 2002$, this task has been carried out exclusively by the authors in the context of a grant agreement between the European Food Safety Authority and the authors. The present document is published complying with the transparency principle to which the Authority is subject. It cannot be considered as an output adopted by the Authority. The European Food Safety Authority reserves its rights, view and position as regards the issues addressed and the conclusions reached in the present document, without prejudice to the rights of the authors. 

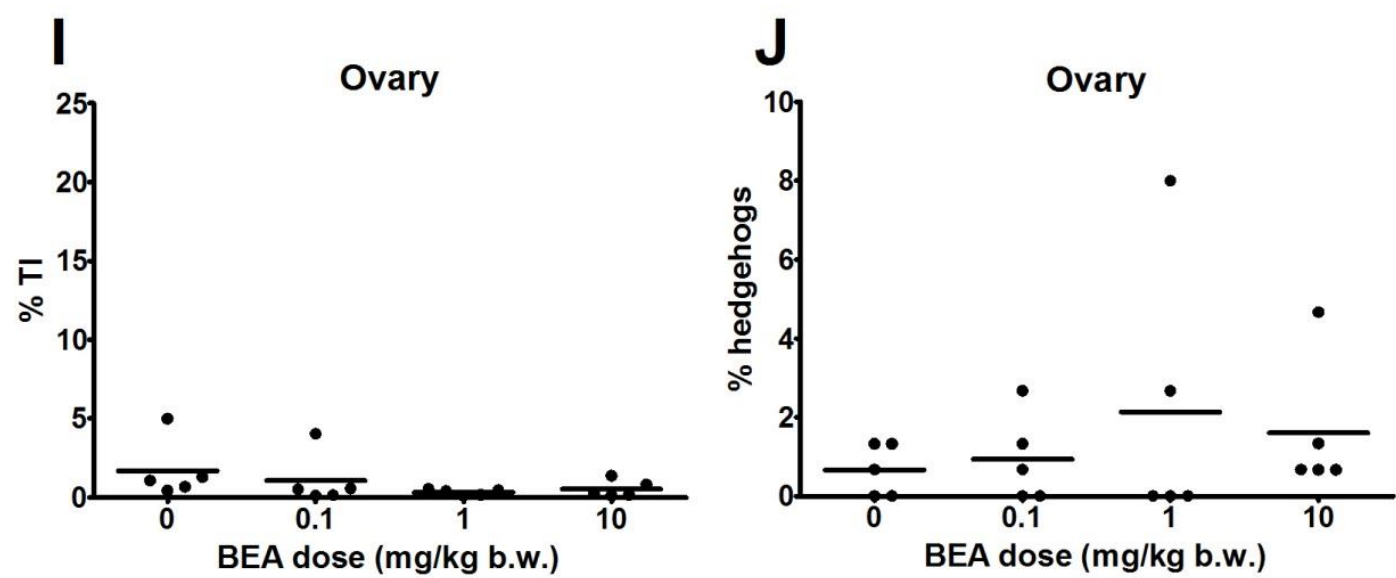

Figure 73: Alkaline Comet assay in different organs/tissues in dams after repeated oral treatments with different doses of BEA ( 0 - olive oil; $0.1,1$ and $10 \mathrm{mg} / \mathrm{kg}$ per day). In A, C, E, G and I dots represent the median of tail intensity obtained for each animal and lines represent the mean of tail intensity for each group. In $B, D, F, H$ and $J$ dots represent the percentage of hedgehogs obtained for each mouse and lines are the mean for each group. 


\subsection{5. $\quad$ ENNB - General toxicity}

\section{Body weight}

Dam body weight was not affected by ENNB treatment in all phases examined (Fig. 74).
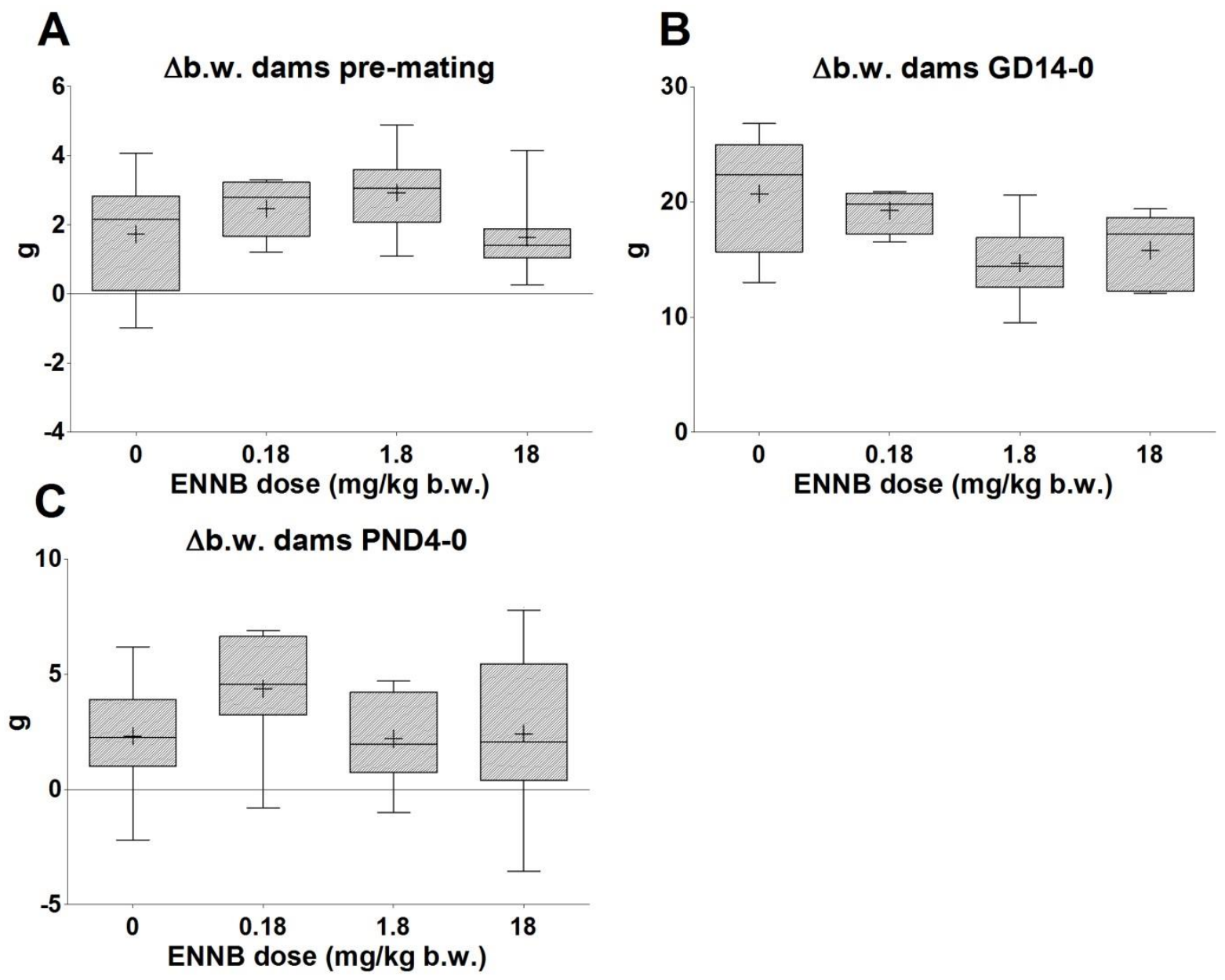

Figure 74: $\quad$ Mean difference in body weight: during pre-mating time $(A)$ during gestation $(B)$ and post-partum (C) of dams orally treated with different doses of ENNB (0 - olive oil+DMSO6\%; 0.18, 1.8 and $18 \mathrm{mg} / \mathrm{kg}$ per day). Levels are reported as interquartile box plots, with whiskers delimiting the 10th and 90th percentiles. " + " indicates the mean value for each experimental group.

\section{Food consumption}

A significant decrease in food consumption was observed in dams of the ENN0.18 group during the pre-mating period, the correlation with the treatment is unclear (fig. 75A). 


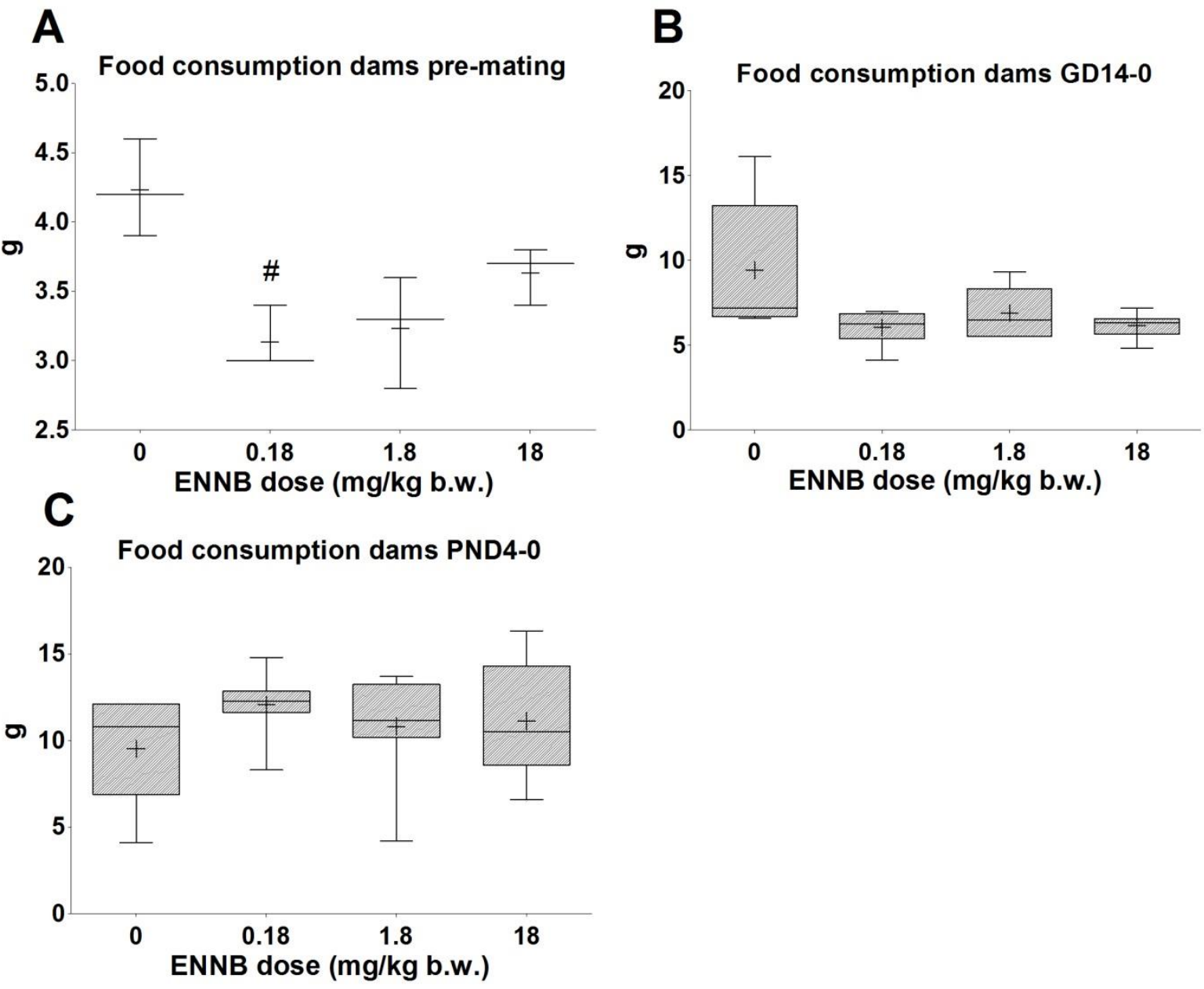

Figure 75: $\quad$ Mean food consumption during pre-mating time (A) during gestation (B) and postpartum (C) of dams orally treated with different doses of ENNB (0 - olive oil+DMSO6\%; $0.18,1.8$ and $18 \mathrm{mg} / \mathrm{kg}$ per day). Levels are reported as interquartile box plots, with whiskers delimiting the 10th and 90th percentiles. "+" indicates the mean value for each experimental group; \# $\mathrm{p}<0.05$

\section{Pups}

Pup and litter weight was not affected by ENNB treatment either at birth or at PND4 (Fig. 76). 

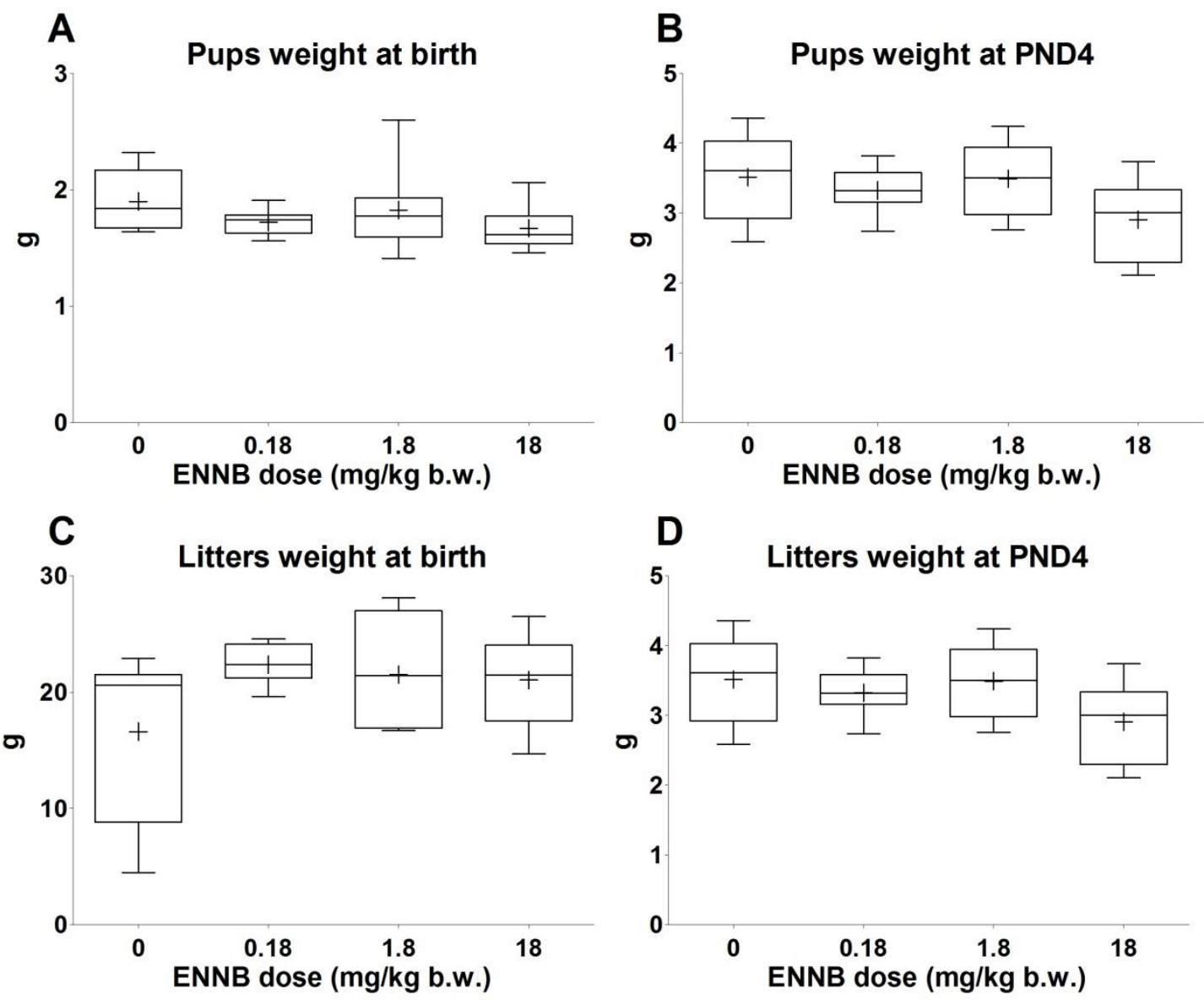

D

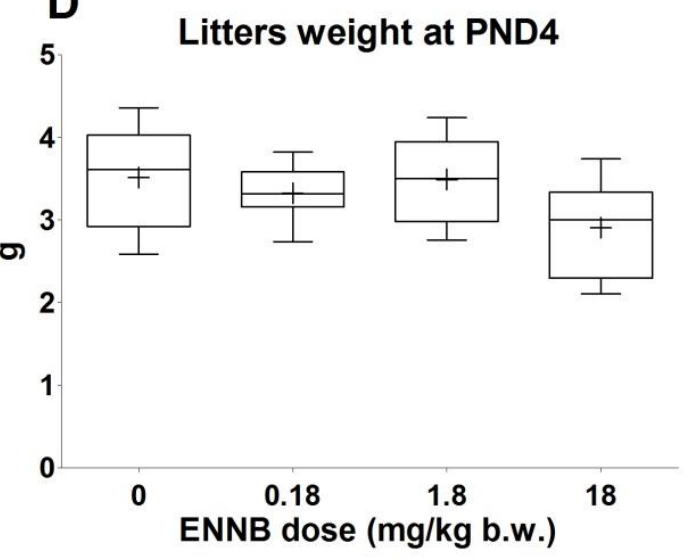

Figure 76: $\quad$ Mean pup weight at birth (A) and at PND4 (B), litter weight at birth (C) and at PND4 (D) born from dams orally treated with different doses of ENNB ( 0 - olive oil+DMSO6\%; $0.18,1.8$ and $18 \mathrm{mg} / \mathrm{kg}$ per day). Levels are reported as interquartile box plots, with whiskers delimiting the 10th and 90th percentiles. " + " indicates the mean value for each experimental group. 
No effect on pup sex ratio was recorded at birth or at PND4 (Fig. 77).
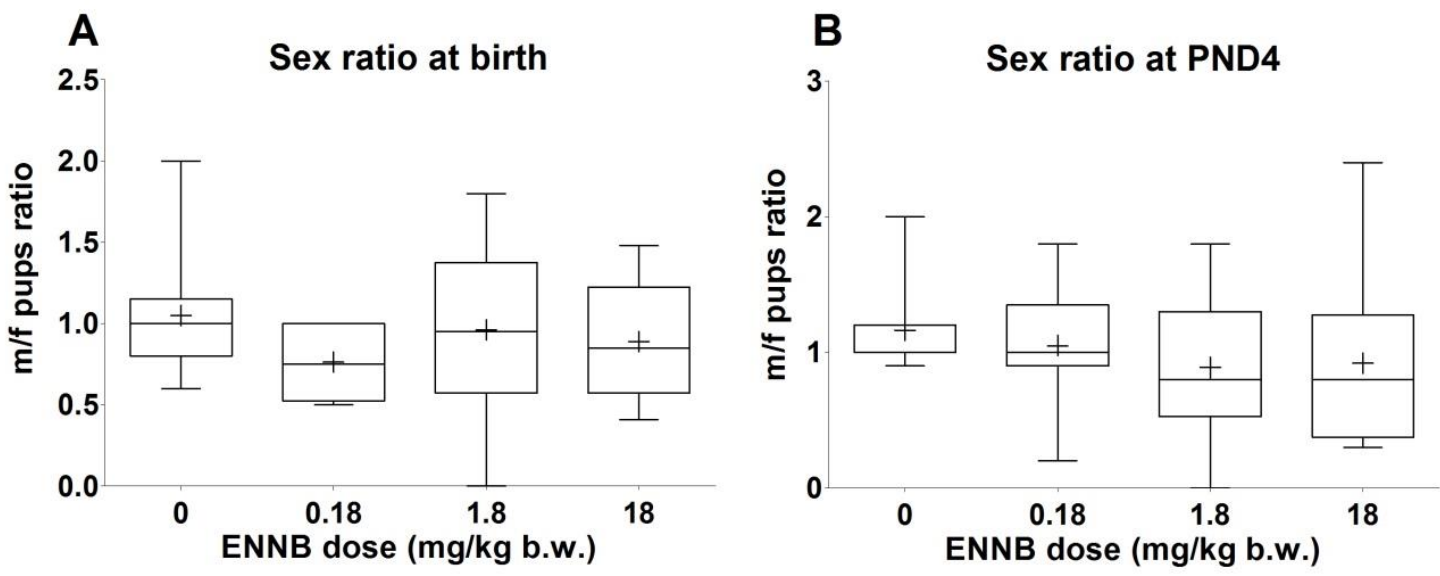

Figure 77: Mean pup sex ratio at birth $(A)$ and at PND4 (B), born of dam mice orally treated with different doses of ENNB ( 0 - olive oil+DMSO6\%; 0.18, 1.8 and $18 \mathrm{mg} / \mathrm{kg}$ per day). Levels are reported as interquartile box plots, with whiskers delimiting the 10th and 90th percentiles. " + " indicates the mean value for each experimental group. 


\section{Organ weight}

Dam organ weight are summarized in Table 34.

Table 34: Absolute and relative organ weights of dams orally treated with different doses of ENNB ( 0 - olive oil+DMSO6\%; 0.18, 1.8 and $18 \mathrm{mg} / \mathrm{kg}$ per day)

\begin{tabular}{|c|c|c|c|c|c|}
\hline \multirow{2}{*}{ ORGAN } & & \multicolumn{4}{|c|}{ Dams } \\
\hline & & Olive oil+DMSO 6\% & ENNB0.18 & ENNB1.8 & ENNB18 \\
\hline & & mean $\pm S D$ & mean $\pm S D$ & mean $\pm S D$ & mean $\pm S D$ \\
\hline LIVER & $\begin{array}{r}N \text { of mice examined } \\
\text { Absolute weight }(\mathrm{g}) \\
\text { Relative weight }(\mathrm{x} 100)\end{array}$ & $\begin{array}{c}8 \\
2.61 \pm 0.53 \\
6.86 \pm 0.68\end{array}$ & $\begin{array}{c}8 \\
3.18 \pm 0.47 \\
7.69 \pm 0.88\end{array}$ & $\begin{array}{c}8 \\
2.89 \pm 0.69 \\
7.66 \pm 1.39\end{array}$ & $\begin{array}{c}10 \\
2.89 \pm 0.42 \\
7.53 \pm 0.77\end{array}$ \\
\hline SPLEEN & $\begin{array}{r}N \text { of mice examined } \\
\text { Absolute weight }(\mathrm{g}) \\
\text { Relative weight }(\mathrm{x} 100)\end{array}$ & $\begin{array}{c}8 \\
0.17 \pm 0.04 \\
0.46 \pm 0.08\end{array}$ & $\begin{array}{c}8 \\
0.19 \pm 0.03 \\
0.46 \pm 0.08\end{array}$ & $\begin{array}{c}8 \\
0.15 \pm 0.05 \\
0.41 \pm 0.12\end{array}$ & $\begin{array}{c}10 \\
0.18 \pm 0.05 \\
0.46 \pm 0.11\end{array}$ \\
\hline MLN & $\begin{array}{r}N \text { of mice examined } \\
\text { Absolute weight }(\mathrm{g}) \\
\text { Relative weight }(\mathrm{x} 100)\end{array}$ & $\begin{array}{c}8 \\
0.02 \pm 0.01 \\
0.04 \pm 0.03\end{array}$ & $\begin{array}{c}8 \\
0.03 \pm 0.01 \\
0.06 \pm 0.04\end{array}$ & $\begin{array}{c}8 \\
0.04 \pm 0.02 \# \\
0.10 \pm 0.05 \# \#\end{array}$ & $\begin{array}{c}10 \\
0.02 \pm 0.01 \\
0.05 \pm 0.03\end{array}$ \\
\hline KIDNEYS & $\begin{array}{r}N \text { of mice examined } \\
\text { Absolute weight }(\mathrm{g}) \\
\text { Relative weight }(\mathrm{x} 100)\end{array}$ & $\begin{array}{c}8 \\
0.48 \pm 0.05 \\
1.29 \pm 0.08\end{array}$ & $\begin{array}{c}8 \\
0.53 \pm 0.07 \\
1.27 \pm 0.14\end{array}$ & $\begin{array}{c}8 \\
0.48 \pm 0.06 \\
1.28 \pm 0.11\end{array}$ & $\begin{array}{c}10 \\
0.48 \pm 0.04 \\
1.27 \pm 0.09\end{array}$ \\
\hline ADRENALS & $\begin{array}{r}N \text { of mice examined } \\
\text { Absolute weight }(\mathrm{g}) \\
\text { Relative weight }(\mathrm{x} 100)\end{array}$ & $\begin{array}{c}8 \\
0.008 \pm 0.003 \\
0.020 \pm 0.009\end{array}$ & $\begin{array}{c}8 \\
0.011 \pm 0.002 \\
0.026 \pm 0.006\end{array}$ & $\begin{array}{c}8 \\
0.025 \pm 0.042 \\
0.053 \pm 0.092\end{array}$ & $\begin{array}{c}10 \\
0.010 \pm 0.002 \\
0.027 \pm 0.007\end{array}$ \\
\hline THYMUS & $\begin{array}{r}N \text { of mice examined } \\
\text { Absolute weight }(\mathrm{g}) \\
\text { Relative weight }(\mathrm{x} 100)\end{array}$ & $\begin{array}{c}8 \\
0.03 \pm 0.01 \\
0.08 \pm 0.04\end{array}$ & $\begin{array}{c}7 \\
0.04 \pm 0.01 \\
0.08 \pm 0.05\end{array}$ & $\begin{array}{c}8 \\
0.08 \pm 0.14 \\
0.23 \pm 0.41\end{array}$ & $\begin{array}{c}9 \\
0.03 \pm 0.01 \\
0.06 \pm 0.03\end{array}$ \\
\hline HEART & $\begin{array}{r}N \text { of mice examined } \\
\text { Absolute weight }(\mathrm{g}) \\
\text { Relative weight }(\mathrm{x} 100)\end{array}$ & $\begin{array}{c}8 \\
0.26 \pm 0.03 \\
0.70 \pm 0.08\end{array}$ & $\begin{array}{c}8 \\
0.23 \pm 0.03 \\
0.55 \pm 0.07 \# \#\end{array}$ & $\begin{array}{c}8 \\
0.21 \pm 0.03 \# \\
0.56 \pm 0.05 \#\end{array}$ & $\begin{array}{c}10 \\
0.25 \pm 0.06 \\
0.65 \pm 0.20\end{array}$ \\
\hline THYROID & $\begin{array}{r}N \text { of mice examined } \\
\text { Absolute weight }(g) \\
\text { Relative weight }(x \quad 100)\end{array}$ & $\begin{array}{c}7 \\
0.005 \pm 0.001 \\
0.013 \pm 0.002\end{array}$ & $\begin{array}{c}6 \\
0.006 \pm 0.001 \\
0.014 \pm 0.003\end{array}$ & $\begin{array}{c}6 \\
0.020 \pm 0.023 \\
0.053 \pm 0.060\end{array}$ & $\begin{array}{c}6 \\
0.004 \pm 0.002 \\
0.012 \pm 0.006\end{array}$ \\
\hline BRAIN & $\begin{array}{r}N \text { of mice examined } \\
\text { Absolute weight }(\mathrm{g}) \\
\text { Relative weight }(\mathrm{x} 100)\end{array}$ & $\begin{array}{c}8 \\
0.44 \pm 0.02 \\
1.19 \pm 0.16\end{array}$ & $\begin{array}{c}8 \\
0.45 \pm 0.03 \\
1.10 \pm 0.12\end{array}$ & $\begin{array}{c}8 \\
0.46 \pm 0.02 \\
1.23 \pm 0.16\end{array}$ & $\begin{array}{c}10 \\
0.47 \pm 0.04 \\
1.22 \pm 0.12\end{array}$ \\
\hline OVARY & $\begin{array}{r}N \text { of mice examined } \\
\text { Absolute weight }(\mathrm{g}) \\
\text { Relative weight }(\mathrm{x} 100)\end{array}$ & $\begin{array}{c}8 \\
0.03 \pm 0.01 \\
0.09 \pm 0.03\end{array}$ & $\begin{array}{c}8 \\
0.06 \pm 0.09 \\
0.16 \pm 0.22\end{array}$ & $\begin{array}{c}8 \\
0.04 \pm 0.01 \\
0.11 \pm 0.03\end{array}$ & $\begin{array}{c}10 \\
0.03 \pm 0.01 \\
0.08 \pm 0.02\end{array}$ \\
\hline
\end{tabular}

\#\# $\mathrm{p}<0.01 ; \# \mathrm{p}<0.05$ 
MLN absolute and relative weight was increased at ENNB1.8, the correlation with the treatment is unclear (Fig. 78E-F).

Heart absolute and relative weight was decreased at ENNB1.8; relative weight was decreased also at ENNB0.18 (Fig. 78M-N). The correlation of both endpoints with the treatment is unclear. 

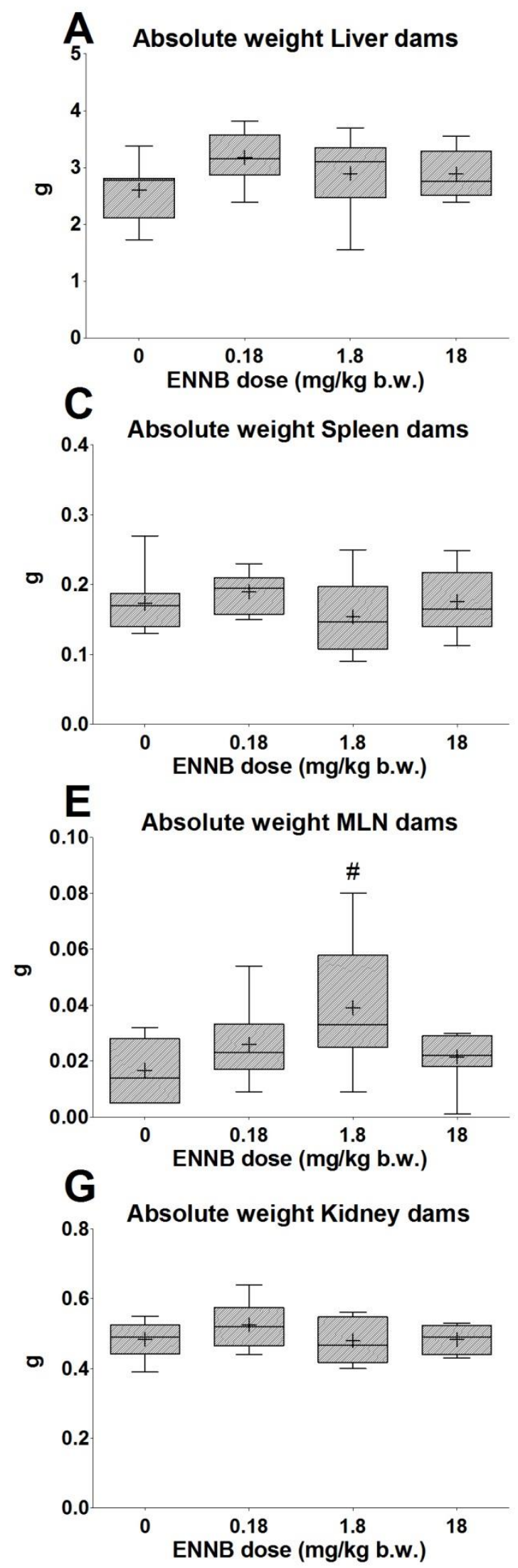

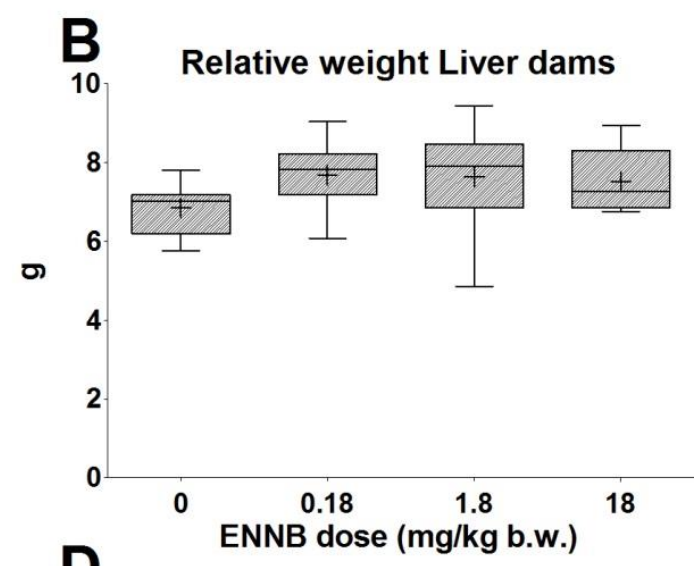

D Relative weight Spleen dams
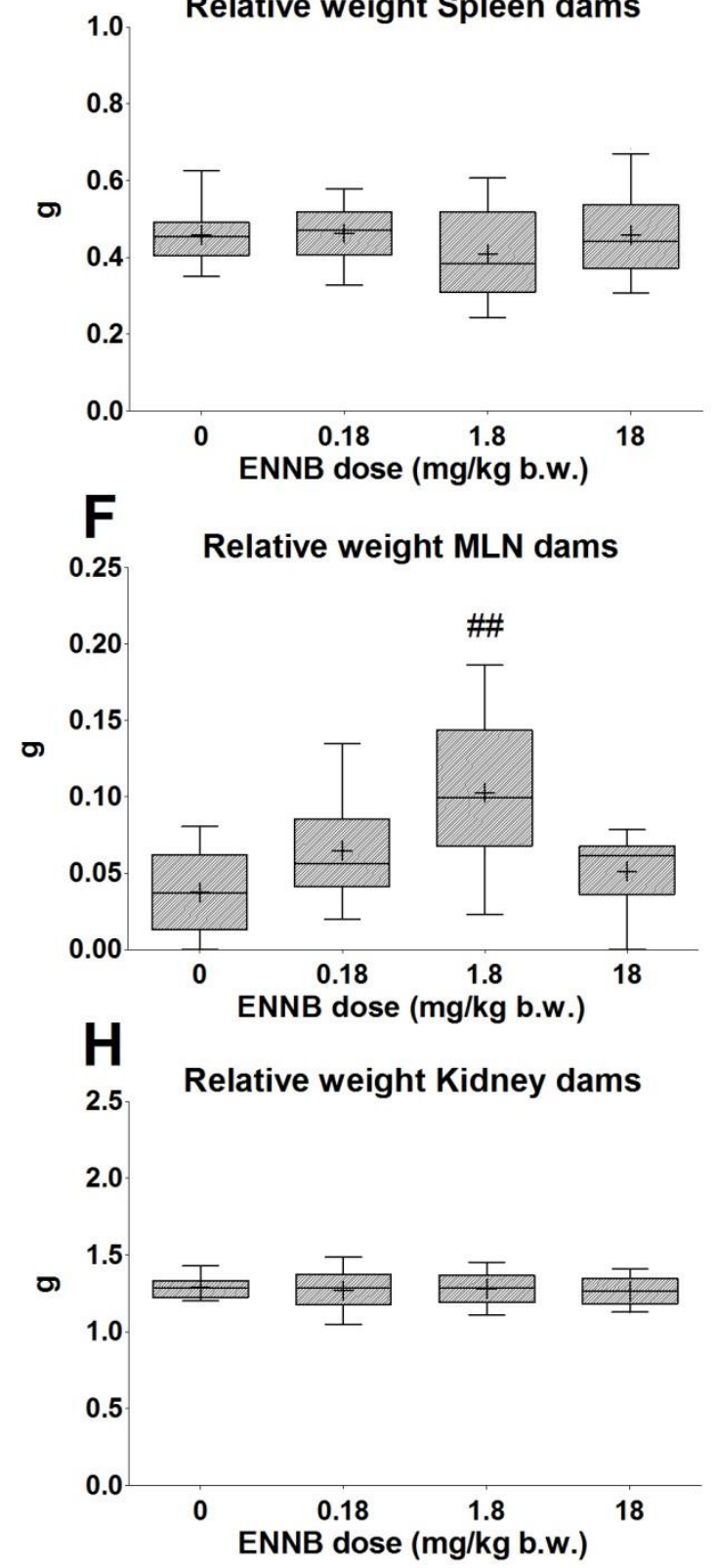

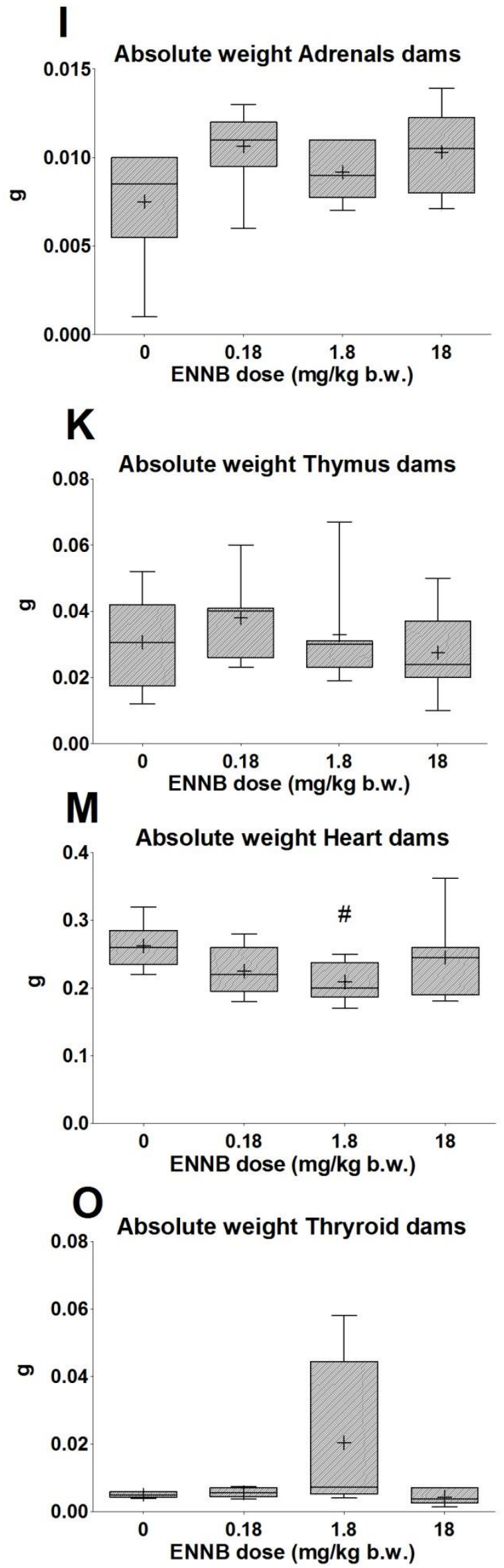
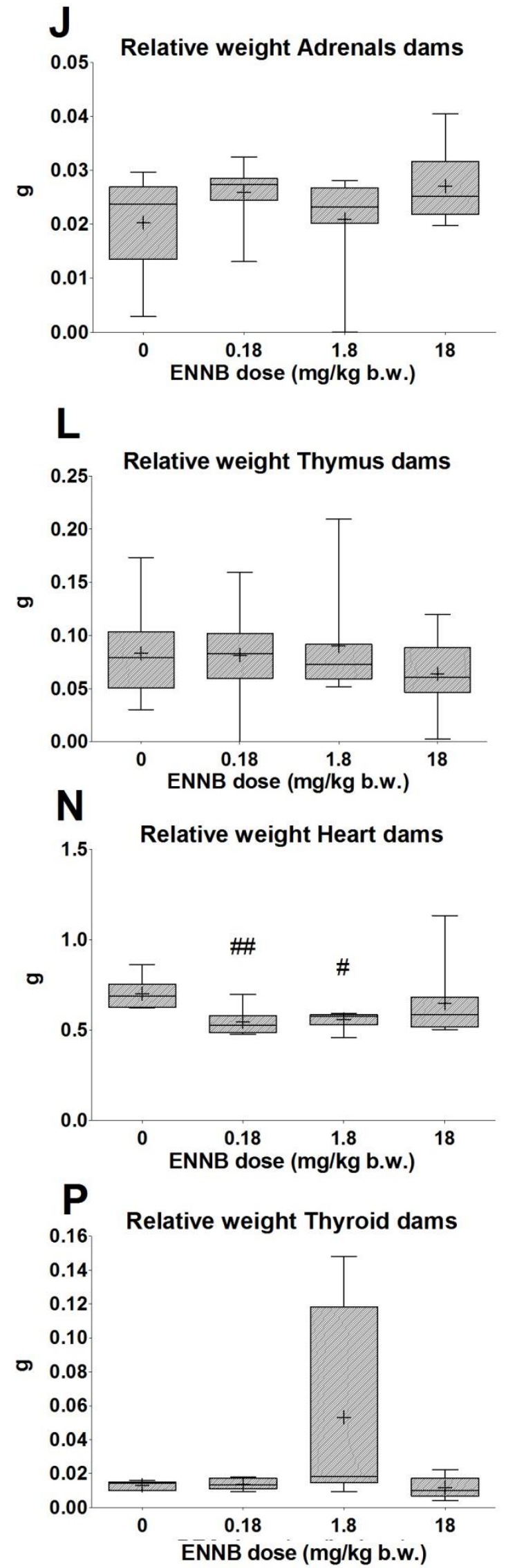

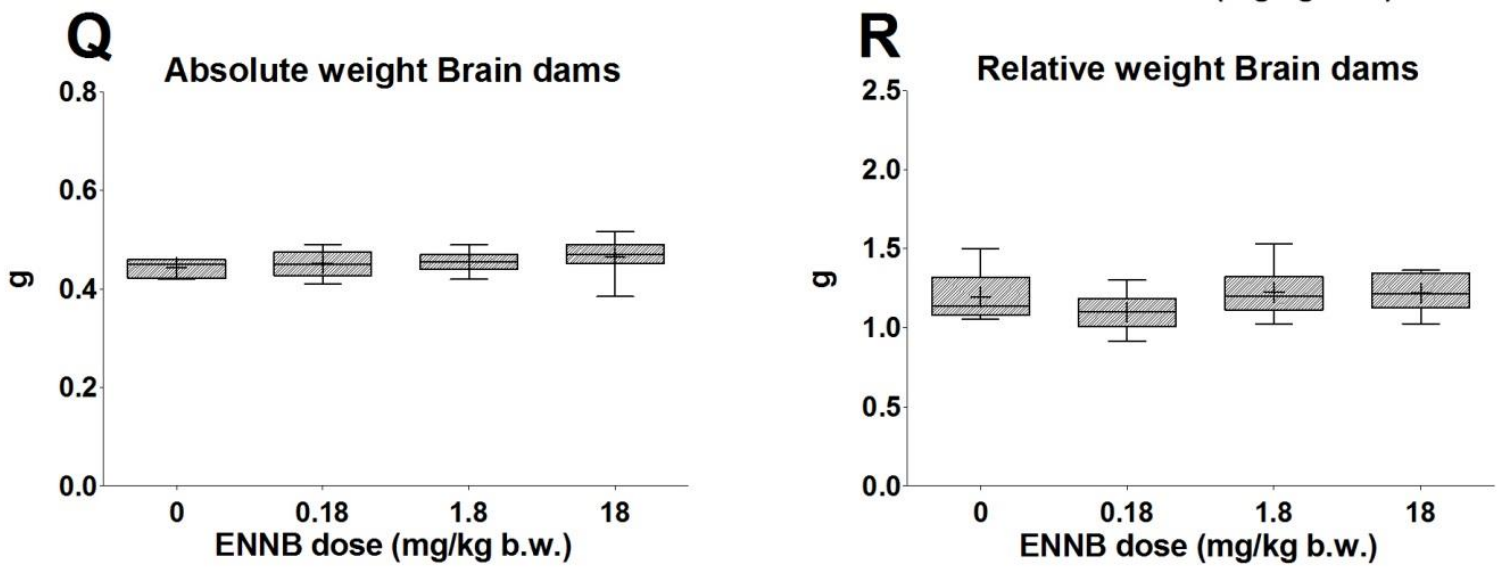

Figure 78: Absolute and relative organ weights of dams orally treated with different doses of ENNB ( 0 - olive oil+DMSO6\%; 0.18, 1.8 and $18 \mathrm{mg} / \mathrm{kg}$ per day). Levels are reported as interquartile box plots, with whiskers delimiting the 10th and 90th percentiles. "+" indicates the mean value for each experimental group; \# $\mathrm{p}<0.05 ; \# \# \mathrm{p}<0.01$.

No effect was observed on dam ovary weight (Fig. 79).
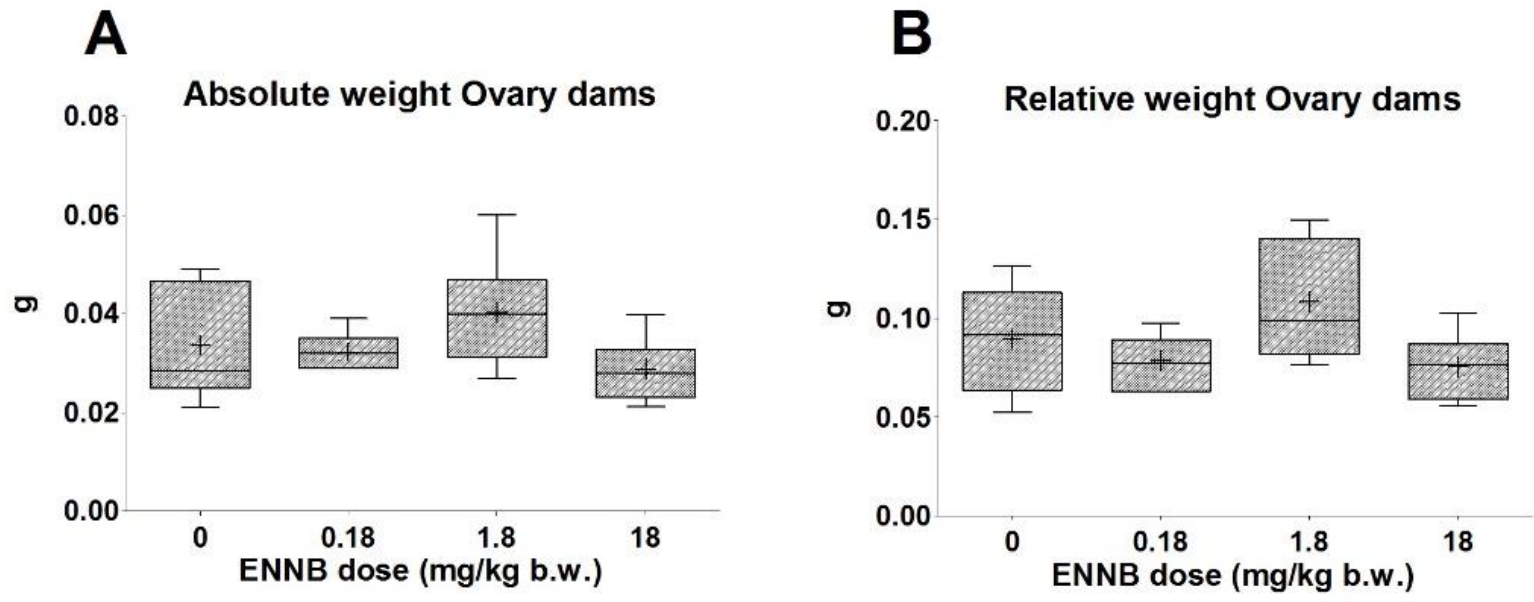

Figure 79: Absolute and relative ovary weights of dams orally treated with different doses of ENNB ( 0 - olive oil+DMSO6\%; 0.18, 1.8 and $18 \mathrm{mg} / \mathrm{kg}$ per day). Levels are reported as interquartile box plots, with whiskers delimiting the 10th and 90th percentiles. " + " indicates the mean value for each experimental group.

\subsubsection{ENNB - Histopathological endpoints}

Table 35 summarized the histopathological examinations in dams treated with ENNB. 
Table 35: Incidence of each histopathological endpoint analyzed in dams orally treated with different doses of ENNB ( 0 - olive oil+DMSO6\%; $0.18,1.8$ and $18 \mathrm{mg} / \mathrm{kg}$ per day).

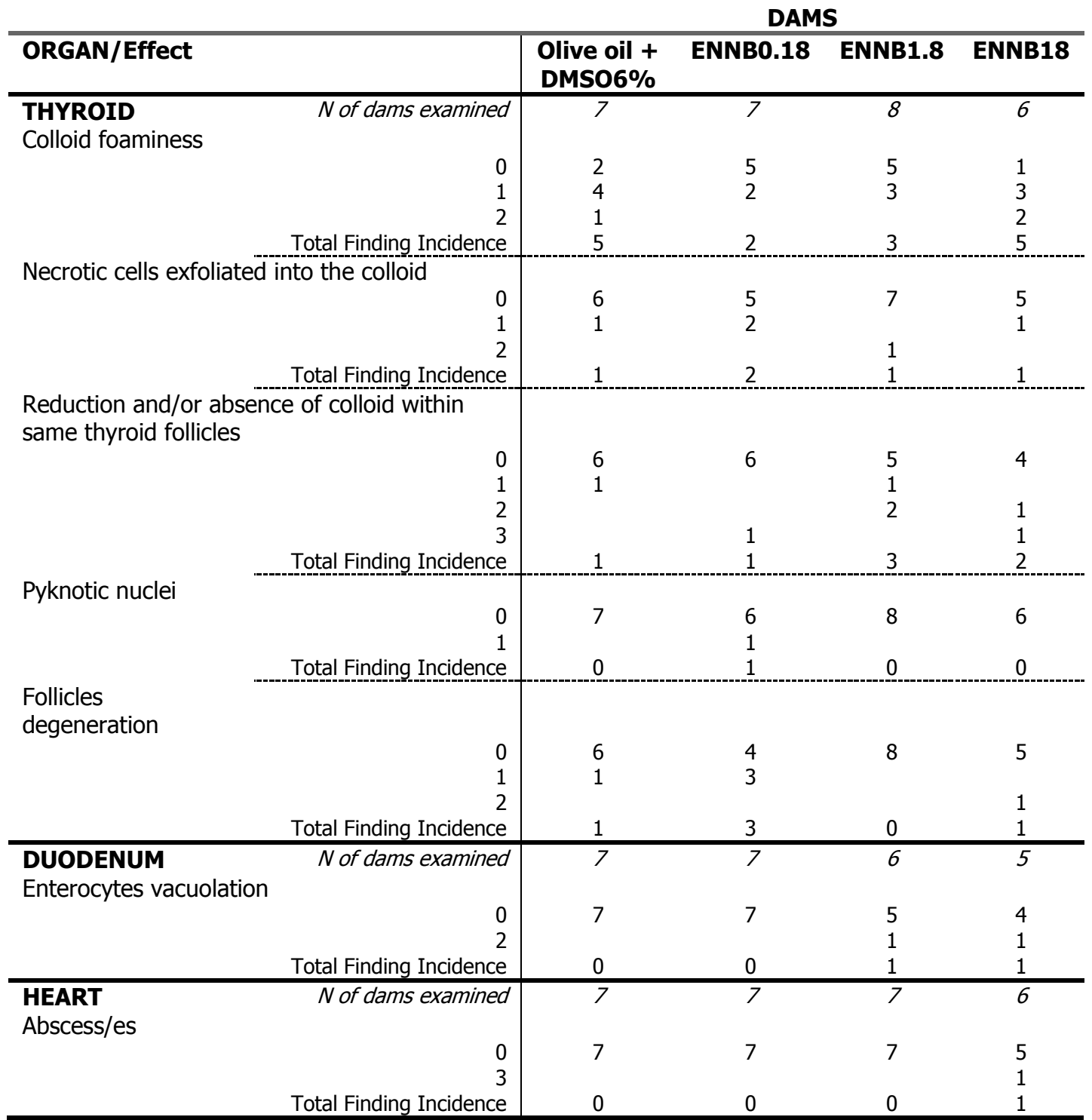

Grade 0: No change; Grade 1: Minimal; Grade 2: Mild; Grade 3: Moderate; Grade 4: Marked. 
Table 35 (continued): Incidence of each histopathological endpoint analyzed in dams orally treated with different doses of ENNB ( 0 - olive oil+DMSO6\%; $0.18,1.8$ and $18 \mathrm{mg} / \mathrm{kg}$ per day).

\begin{tabular}{|c|c|c|c|c|}
\hline \multirow[b]{2}{*}{ ORGAN/Effect } & \multicolumn{4}{|c|}{ DAMS } \\
\hline & $\begin{array}{l}\text { Olive oil + } \\
\text { DMSO6\% }\end{array}$ & ENNB0.18 & ENNB1.8 & ENNB18 \\
\hline$N$ of dams examined & 7 & 7 & 7 & 6 \\
\hline $\begin{array}{r}0 \\
1 \\
2 \\
3 \\
\text { Total Finding Incidence }\end{array}$ & $\begin{array}{l}2 \\
1 \\
3 \\
1 \\
5\end{array}$ & $\begin{array}{l}2 \\
4 \\
1 \\
7\end{array}$ & $\begin{array}{l}1 \\
4 \\
2 \\
6\end{array}$ & $\begin{array}{l}1 \\
1 \\
4 \\
5\end{array}$ \\
\hline $\begin{array}{r}0 \\
1 \\
2 \\
\text { Total Finding Incidence }\end{array}$ & 7 & $\begin{array}{l}5 \\
1 \\
1 \\
2\end{array}$ & 7 & 6 \\
\hline $\begin{array}{r}0 \\
2 \\
\text { Total Finding Incidence }\end{array}$ & $\begin{array}{l}5 \\
2 \\
2\end{array}$ & 7 & $\begin{array}{l}7 \\
0\end{array}$ & $\begin{array}{l}6 \\
0\end{array}$ \\
\hline $\begin{array}{r}0 \\
1 \\
\text { Total Finding Incidence }\end{array}$ & $\begin{array}{l}5 \\
2 \\
2\end{array}$ & $\begin{array}{l}7 \\
0\end{array}$ & $\begin{array}{l}7 \\
0\end{array}$ & $\begin{array}{l}6 \\
0\end{array}$ \\
\hline $\begin{array}{lr}\text { SPLEEN } & N \text { of dams examined } \\
\text { Extramedullary haematopoiesis } & 1 \\
& 2 \\
& 3 \\
& \text { Total Finding Incidence } \\
\end{array}$ & $\begin{array}{l}2 \\
1 \\
4 \\
7\end{array}$ & $\begin{array}{l}7 \\
7 \\
\end{array}$ & $\begin{array}{l}5 \\
2 \\
7\end{array}$ & $\begin{array}{l}6 \\
1 \\
2 \\
3 \\
6\end{array}$ \\
\hline $\begin{array}{r}\text { Nof dams examined } \\
\\
0 \\
1 \\
\text { Total Finding Incidence } \\
\end{array}$ & $\begin{array}{l}3 \\
2 \\
2\end{array}$ & $\begin{array}{l}5 \\
2 \\
2\end{array}$ & $\begin{array}{l}5 \\
1 \\
1\end{array}$ & $\begin{array}{l}3 \\
2 \\
2\end{array}$ \\
\hline $\begin{array}{r}N \text { of dams examined } \\
0 \\
1 \\
2 \\
3 \\
\text { Total Finding Incidence }\end{array}$ & $\begin{array}{l}7 \\
1 \\
5 \\
1 \\
6\end{array}$ & $\begin{array}{l}3 \\
4 \\
7\end{array}$ & $\begin{array}{l}3 \\
3 \\
6\end{array}$ & $\begin{array}{l}6 \\
2 \\
2 \\
2 \\
4\end{array}$ \\
\hline $\begin{array}{rr}\text { Subcapsular cell hyperplasia } & 0 \\
1 \\
\text { Total Finding Incidence }\end{array}$ & $\begin{array}{l}6 \\
1 \\
1\end{array}$ & $\begin{array}{l}5 \\
2 \\
2\end{array}$ & $\begin{array}{l}4 \\
2 \\
2\end{array}$ & $\begin{array}{l}5 \\
1 \\
1\end{array}$ \\
\hline $\begin{array}{l}\text { OVARY } \\
\text { Follicle with apoptosis in granulosa cell } \\
\\
\\
\text { Total Finding Incidence }\end{array}$ & $\begin{array}{l}1 \\
1 \\
0\end{array}$ & $\begin{array}{l}3 \\
3 \\
0\end{array}$ & $\begin{array}{l}3 \\
1 \\
2 \\
2\end{array}$ & $\begin{array}{l}2 \\
2\end{array}$ \\
\hline $\begin{array}{r}\text { Degeneration follicles with degenerate oocyte } \\
0 \\
\text { Total Finding Incidence }\end{array}$ & $\begin{array}{l}1 \\
0\end{array}$ & $\begin{array}{l}3 \\
0\end{array}$ & $\begin{array}{l}3 \\
0\end{array}$ & $\begin{array}{l}2 \\
0\end{array}$ \\
\hline
\end{tabular}

Grade 0: No change; Grade 1: Minimal; Grade 2: Mild; Grade 3: Moderate; Grade 4: Marked. 
Table 35 (continued): Incidence of each histopathological endpoint analyzed in dams orally treated with different doses of ENNB ( 0 - olive oil+DMSO6\%; $0.18,1.8$ and $18 \mathrm{mg} / \mathrm{kg}$ per day).

\begin{tabular}{|c|c|c|c|c|c|}
\hline BRAIN & N of dams examined & 7 & 7 & 7 & 6 \\
\hline & Total Finding Incidence & $\begin{array}{l}5 \\
2 \\
2\end{array}$ & $\begin{array}{l}7 \\
0 \\
\end{array}$ & $\begin{array}{l}7 \\
0\end{array}$ & 6 \\
\hline Spongiosis & $\begin{array}{r}0 \\
2 \\
3 \\
4 \\
\text { Total Finding Incidence }\end{array}$ & $\begin{array}{l}1 \\
3 \\
2 \\
1 \\
6\end{array}$ & $\begin{array}{l}1 \\
1 \\
1 \\
4 \\
6\end{array}$ & $\begin{array}{l}4 \\
3 \\
7\end{array}$ & $\begin{array}{l}2 \\
2 \\
1 \\
1 \\
4\end{array}$ \\
\hline
\end{tabular}

Grade 0: No change; Grade 1: Minimal; Grade 2: Mild; Grade 3: Moderate; Grade 4: Marked. 
Histopathological and histomorphometrical analyses of thyroid did not evidence any alteration in dams (Table 35 and Fig. 80).
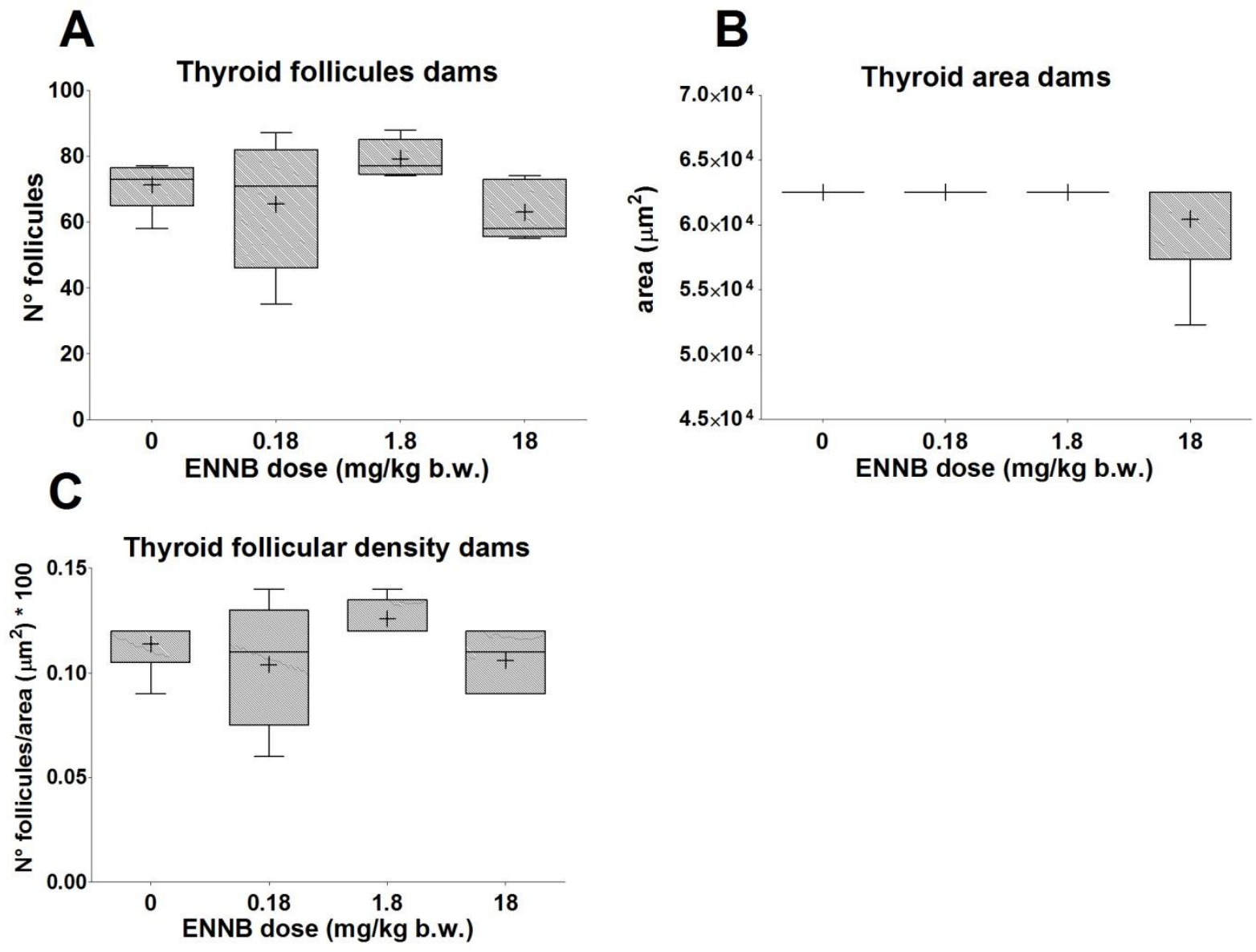

Figure 80: Histomorphometrical analysis of number of follicles $(A)$ and thyroid area (B); follicular density (C) in dams orally treated with different doses ENNB ( 0 - olive oil+DMSO6\%; 0.18 , 1.8 and $18 \mathrm{mg} / \mathrm{kg}$ per day). Levels are reported as interquartile box plots, with whiskers delimiting the 10th and 90th percentiles. " + " indicates the mean value for each experimental group

No histopathological alterations were observed in dam duodenum, heart and liver (Table 35).

In spleen, no histopathological effects were observed (Table 35); however, histomorphometrical analysis showed a significant increase of white pulp in dams treated with ENN18; consequently, the red to white pulp ratio was significantly decreased in the same group (Fig. $81 \mathrm{~B}-\mathrm{C}$ ). 
A
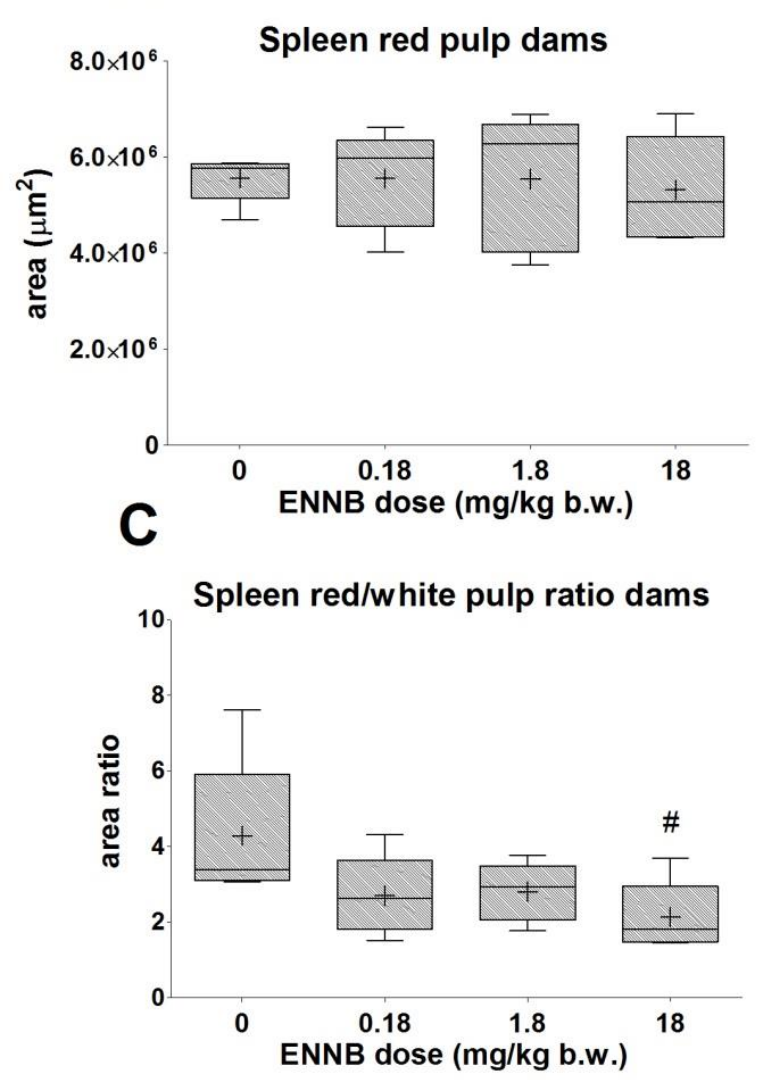

B

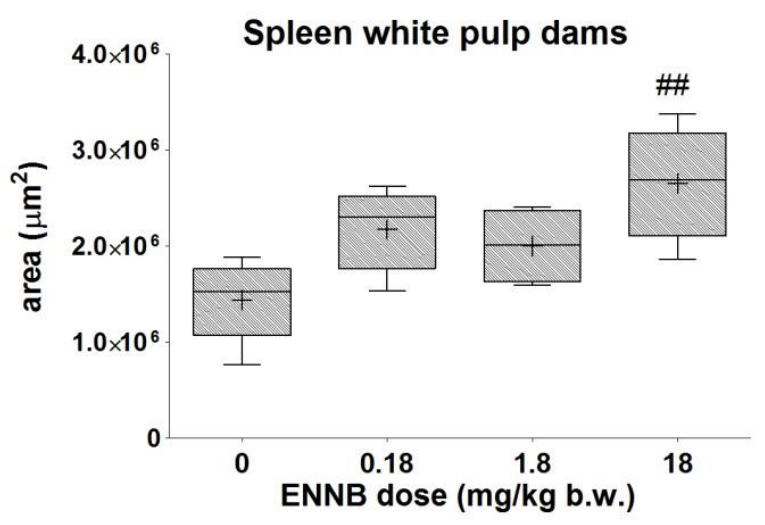

Figure 81: Histomorphometrical analysis of spleen red pulp area (A) and white pulp area (B); ratio of the red to white areas $(C)$ in dams orally treated with different doses of ENNB ( 0 olive oil+DMSO6\%; 0.18, 1.8 and $18 \mathrm{mg} / \mathrm{kg}$ per day). Levels are reported as interquartile box plots, with whiskers delimiting the 10th and 90th percentiles. "+" indicates the mean value for each experimental group; \#\# $\mathrm{p}<0.01$.

No histopathological effect was recorded in kidney (Table 35).

Similarly, no effect was recorded on thymus either by histopathological and histomorphometrical analyses (Table 35 and Fig. 82). 
A
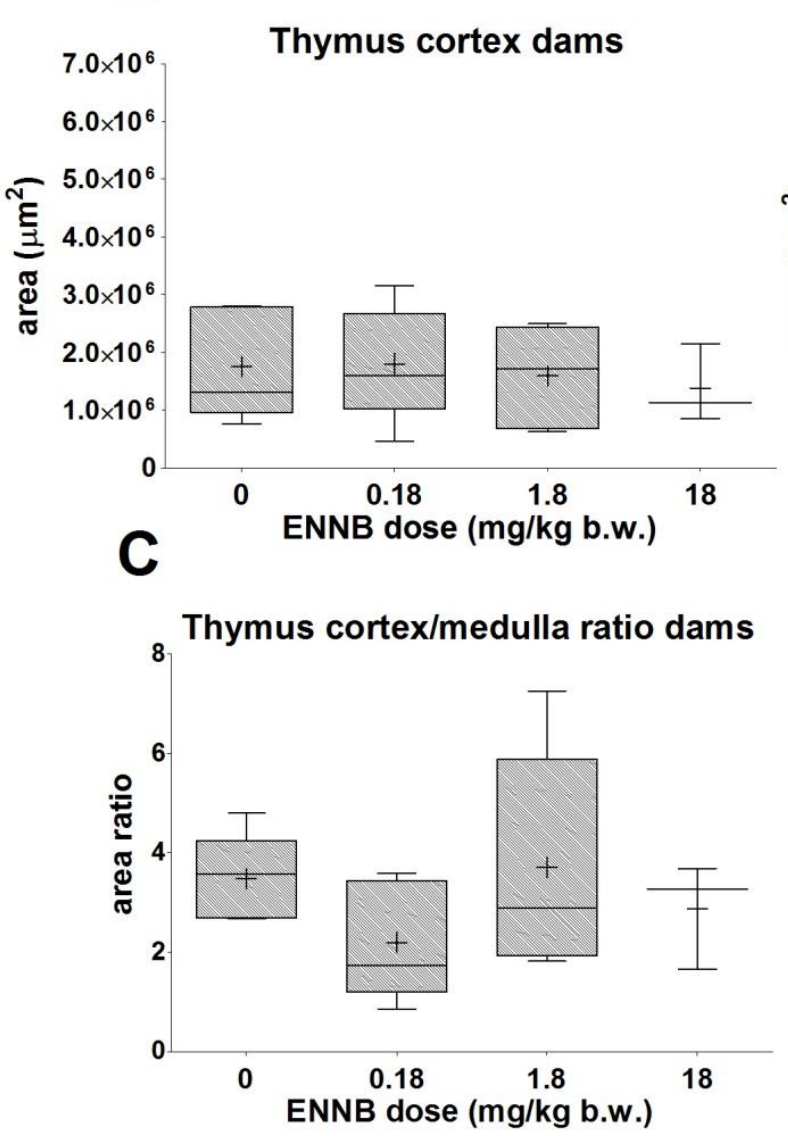

B

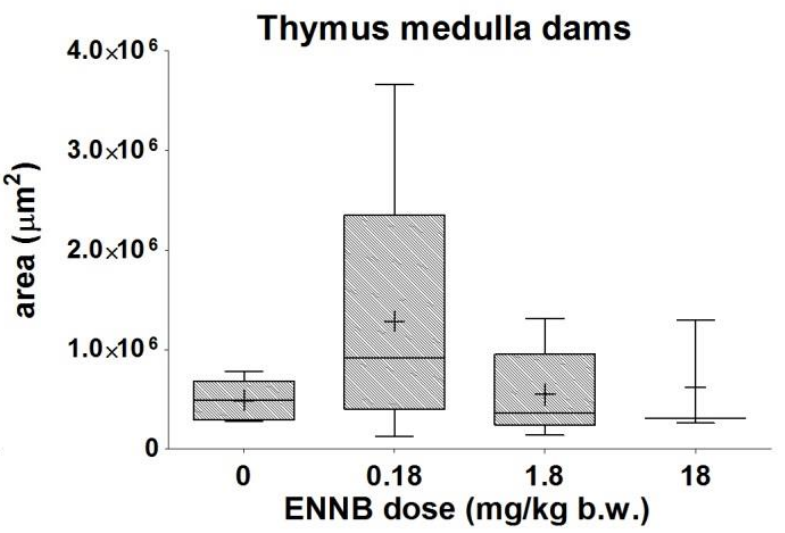

Figure 82: Histomorphometrical analysis of cortex $(A)$ and medulla (B) thymus areas; ratio of the cortex to medulla areas (C) in dams orally treated with different doses of ENNB ( 0 - olive oil+DMSO6\%; 0.18, 1.8 and $18 \mathrm{mg} / \mathrm{kg}$ per day). Levels are reported as interquartile box plots, with whiskers delimiting the 10th and 90th percentiles. "+" indicates the mean value for each experimental group.

No effects on histopathological and histomorphometrical adrenal endpoints were observed in dams (Table 35 and Fig. 83). 
A
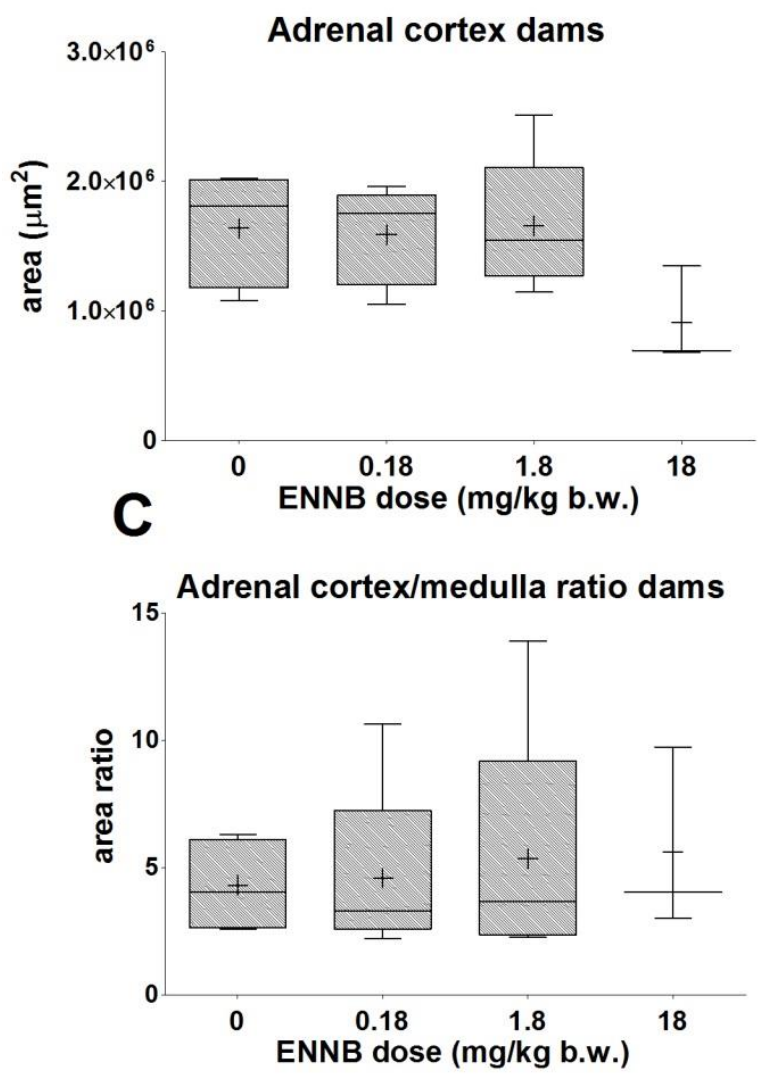

B

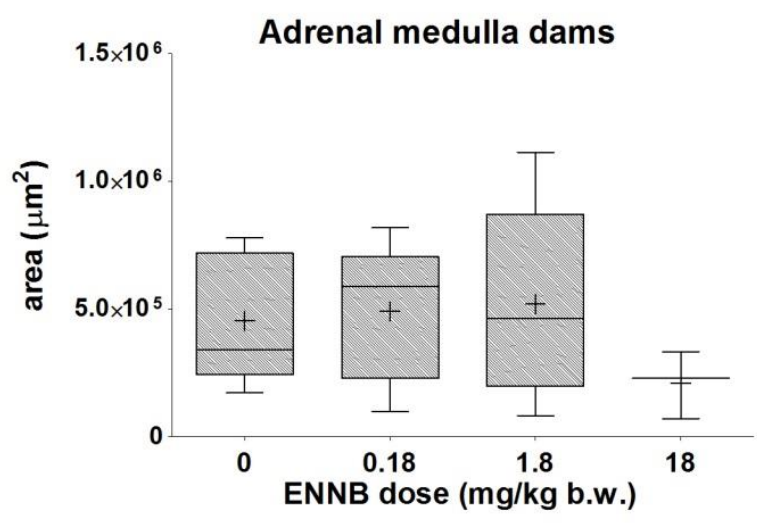

Figure 83: Histomorphometrical analysis of cortex (A) and medulla (B) adrenal areas; ratio of the cortex to medulla adrenal areas (C) in in dams orally treated with different doses of ENNB ( 0 - olive oil+DMSO6\%; $0.18,1.8$ and $18 \mathrm{mg} / \mathrm{kg}$ per day). Levels are reported as interquartile box plots, with whiskers delimiting the 10th and 90th percentiles. " + " indicates the mean value for each experimental group.

Some ovaries of dams treated with ENNB were missed during tissue slide preparation. Thus, histopathological analysis was performed made on the available samples. No histomorphometrical analysis were performed.

\subsection{7. $\quad$ ENNB - Biomarker endpoints}

\section{Liver and kidney biomarkers}

No effect was recorded in dams for both liver and kidney biomarkers (Fig. 84). 

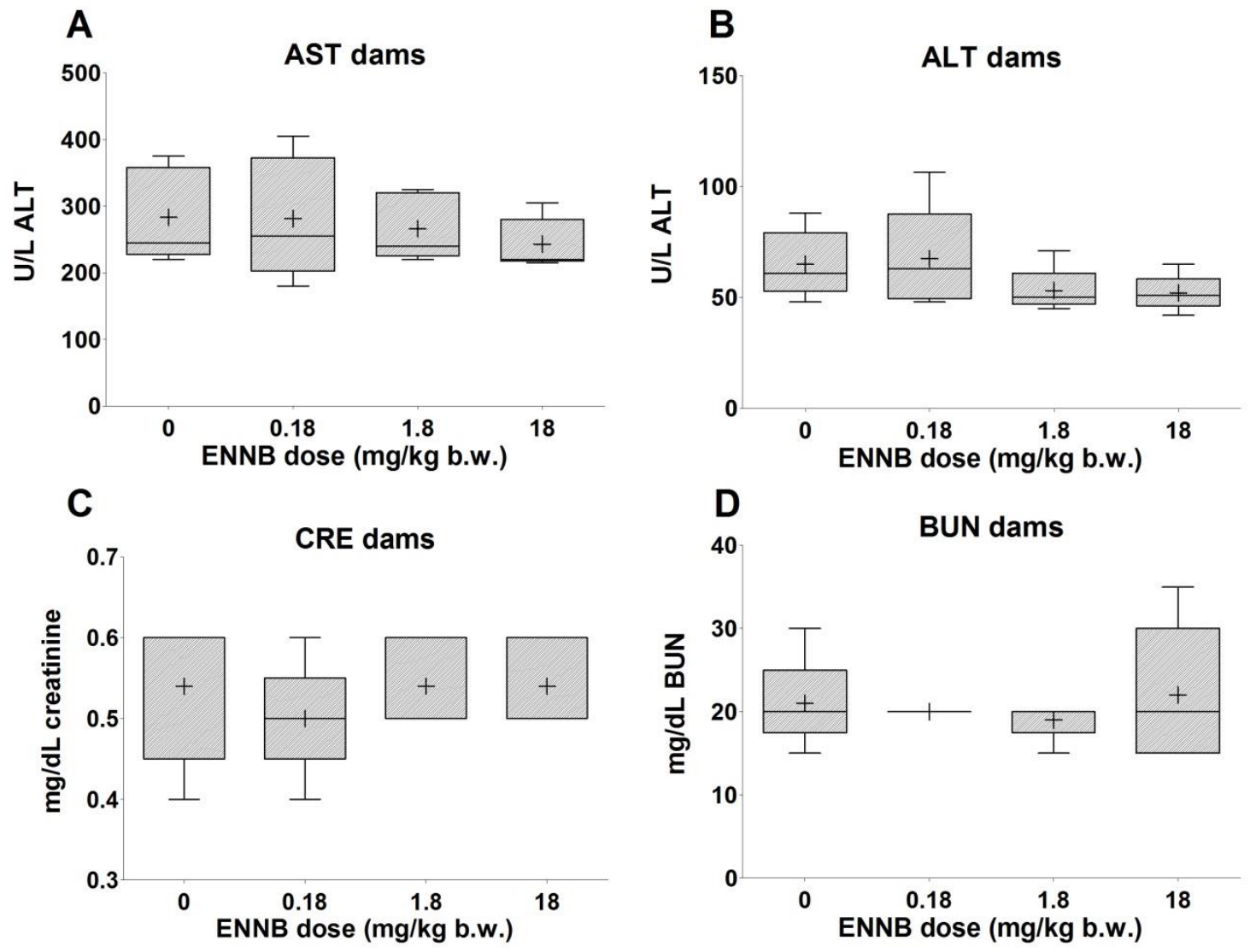

Figure 84: Serum levels of the liver biomarkers aspartate aminotransferase $(A)$ and alanine aminotransferase (B), and of the kidney biomarkers creatinine (C) and blood urea nitrogen (D) in dam mice orally treated with different doses of ENNB ( 0 - olive oil+DMSO6\%; 0.18 , 1.8 and $18 \mathrm{mg} / \mathrm{kg}$ per day). Levels are reported as interquartile box plots, with whiskers delimiting the 10th and 90th percentiles. " + " indicates the mean value for each experimental group

\section{Hormone serum levels}

No effects were present in hormone serum levels of dams exposed to ENNB (Fig. 85). 

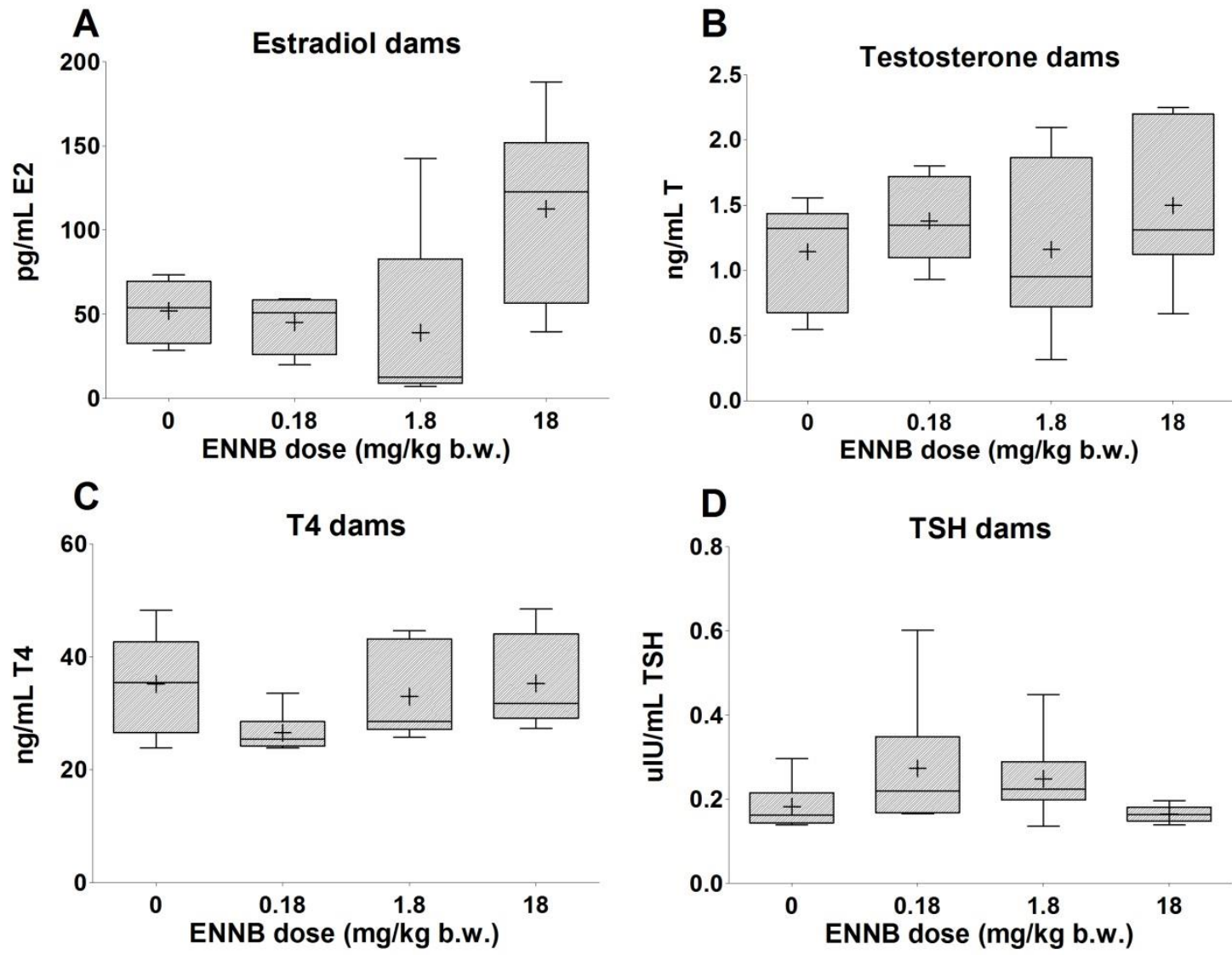

Figure 85: $\quad$ Serum levels of the hormones estradiol (A), testosterone (B), T4 (C) and TSH (D) in dams orally treated with different doses of ENNB ( 0 - olive oil+DMSO6\%; $0.18,1.8$ and 18 $\mathrm{mg} / \mathrm{kg}$ per day). Levels are reported as interquartile box plots, with whiskers delimiting the 10 th and 90th percentiles. " + " indicates the mean value for each experimental group

\section{Oxidative stress markers in brain}

Brain ROS was significantly decreased in ENNB1.8 dams, the correlation with the treatment is unclear (Fig. 86A). No effect was recorded on GSH brain level (Fig. 86B). 

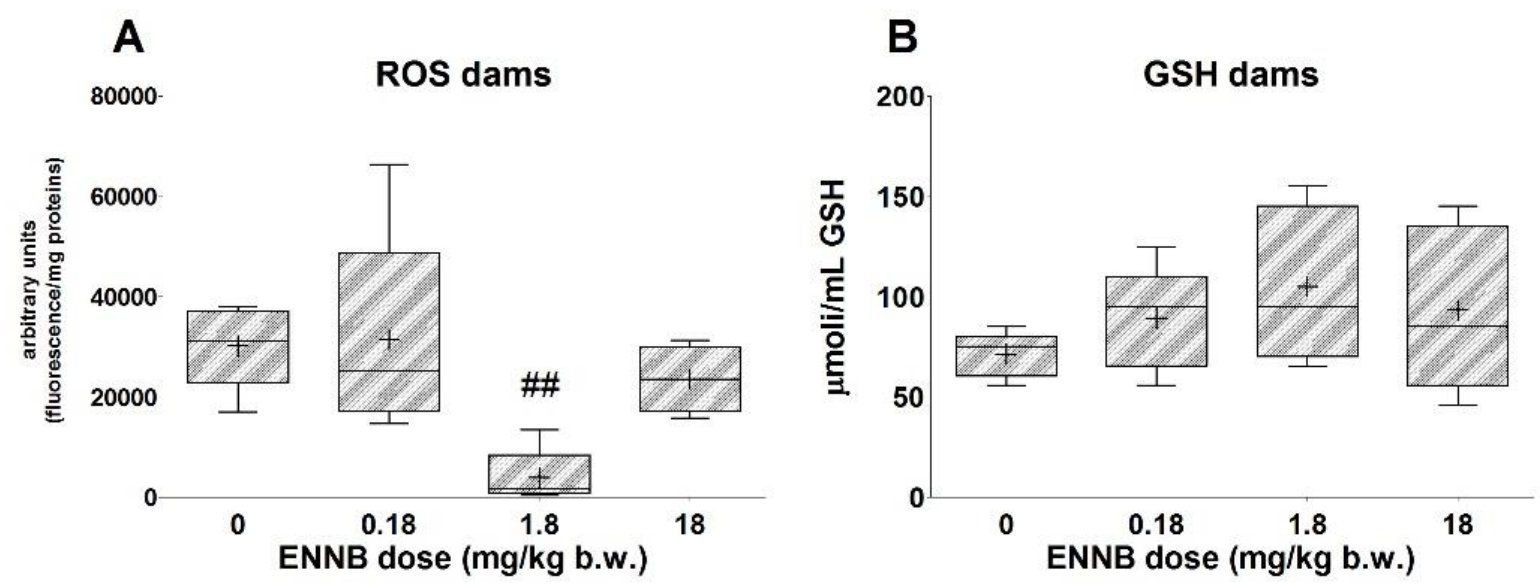

Figure 86: Brain levels of ROS (A) and GSH (B) in dams orally treated with different doses of ENNB ( 0 - olive oil+DMSO6\%; 0.18, 1.8 and $18 \mathrm{mg} / \mathrm{kg}$ per day). Levels are reported as interquartile box plots, with whiskers delimiting the 10th and 90th percentiles. " + " indicates the mean value for each experimental group; \#\# $p<0.01$

\subsubsection{ENNB - Genotoxicity endpoints}

\section{Comet assay}

Results obtained by Comet assay in dams are reported in Table 36 and Figure 87. Data relative to the positive CTRL groups are those reported in the corresponding table relative to experiment with BEA repeated dose oral toxicity study.

No induction of DNA damage was observed. A significant higher amount of \% hedgehodgs was observed in kidney and in ovary in the ENNB18 group. The significant increase of \% hedgehogs in kidney is not to be considered a strong indication of ENNB-related toxicity. In fact, the effect was not massive, and the etiology of hedgehogs is uncertain (OECD TC $489^{1}$ ). Conversely, the significant increase of \% hedgehogs in ovary appeared to be more substantial and might be an indication of an alteration of ovary tissue.

\footnotetext{
${ }^{1}$ OECD, Test No. 489, 2014: In Vivo Mammalian Alkaline Comet Assay, OECD Publishing, Paris. 
Table 36: Alkaline Comet assay in different organs/tissues of dams after repeated oral treatments with different doses of ENNB ( 0 - olive oil+DMSO6\%; $0.18,1.8$ and $18 \mathrm{mg} / \mathrm{kg}$ per day). For each experimental group the mean of median and standard error of the values obtained from each animal for tail length (Tail-L), tail intensity (\% TI) and Olive Tail Moment (OTM) are reported. The mean \% hedgehogs for each group is shown.

\begin{tabular}{lllccc}
\hline Tissue/organ & Treatment & $\begin{array}{c}\text { Tail-L } \\
\text { mean } \pm \text { SE }\end{array}$ & $\begin{array}{c}\text { \% TI } \\
\text { mean } \pm \text { SE }\end{array}$ & $\begin{array}{c}\text { OTM } \\
\text { mean } \pm \text { SE }\end{array}$ & $\begin{array}{c}\text { \% hedgehogs } \\
\text { mean } \pm \text { SE }\end{array}$ \\
\hline Blood & olive oil + DMSO6\% & $0.023 \pm 0.023$ & $0.310 \pm 0.036$ & $0.022 \pm 0.002$ & $0.000 \pm 0.000$ \\
& ENNB0.18 & $0.023 \pm 0.023$ & $0.432 \pm 0.086$ & $0.029 \pm 0.006$ & $0.000 \pm 0.000$ \\
& ENNB1.8 & $0.256 \pm 0.125$ & $0.394 \pm 0.069$ & $0.031 \pm 0.007$ & $0.268 \pm 0.164$ \\
& ENNB18 & $0.000 \pm 0.000$ & $0.206 \pm 0.046$ & $0.019 \pm 0.003$ & $0.000 \pm 0.000$ \\
\hline Liver & olive oil + DMSO6\% & $1.361 \pm 0.231$ & $0.641 \pm 0.197$ & $0.070 \pm 0.022$ & $1.133 \pm 0.507$ \\
& ENNB0.18 & $1.245 \pm 0.482$ & $0.879 \pm 0.487$ & $0.094 \pm 0.057$ & $0.533 \pm 0.341$ \\
& ENNB1.8 & $0.372 \pm 0.105$ & $0.171 \pm 0.034$ & $0.018 \pm 0.003$ & $0.867 \pm 0.282$ \\
& ENNB18 & $1.296 \pm 0.554$ & $0.527 \pm 0.227$ & $0.057 \pm 0.026$ & $1.400 \pm 0.608$ \\
\hline Kidney & olive oil + DMSO6\% & $0.302 \pm 0.087$ & $0.523 \pm 0.141$ & $0.040 \pm 0.012$ & $0.533 \pm 0.389$ \\
& ENNB0.18 & $0.279 \pm 0.108$ & $0.984 \pm 0.284$ & $0.068 \pm 0.021$ & $0.133 \pm 0.133$ \\
& ENNB1.8 & $0.628 \pm 0.375$ & $0.877 \pm 0.388$ & $0.077 \pm 0.040$ & $0.821 \pm 0.405$ \\
& ENNB18 & $0.185 \pm 0.0593$ & $0.224 \pm 0.019$ & $0.020 \pm 0.002$ & $2.004 \pm 0.470 \#$ \\
\hline Duodenum & olive oil + DMSO6\% & $0.722 \pm 0.231$ & $1.108 \pm 0.334$ & $0.087 \pm 0.025$ & $0.000 \pm 0.000$ \\
& ENNB0.18 & $0.780 \pm 0.436$ & $2.033 \pm 0.962$ & $0.158 \pm 0.082$ & $0.133 \pm 0.133$ \\
& ENNB1.8 & $0.338 \pm 0.080$ & $0.517 \pm 0.119$ & $0.041 \pm 0.008$ & $0.402 \pm 0.267$ \\
& ENNB18 & $0.279 \pm 0.101$ & $0.427 \pm 0.112$ & $0.037 \pm 0.010$ & $1.333 \pm 0.699$ \\
\hline Ovary & olive oil + DMSO6\% & $0.421 \pm 0.079$ & $0.523 \pm 0.098$ & $0.039 \pm 0.008$ & $0.267 \pm 0.163$ \\
& ENNB0.18 & $1.140 \pm 0.334$ & $1.663 \pm 0.514$ & $0.148 \pm 0.053$ & $3.002 \pm 1.676$ \\
& ENNB1.8 & $2.350 \pm 1.886$ & $2.269 \pm 1.833$ & $0.228 \pm 0.194$ & $3.048 \pm 1.479$ \\
& ENNB18 & $1.095 \pm 0.565$ & $0.855 \pm 0.485$ & $0.080 \pm 0.050$ & $10.108 \pm 3.167 \# \#$ \\
\hline
\end{tabular}

\footnotetext{
${ }^{\#}=p<0.05 ;{ }^{\# \#}=p<0.01$
} 

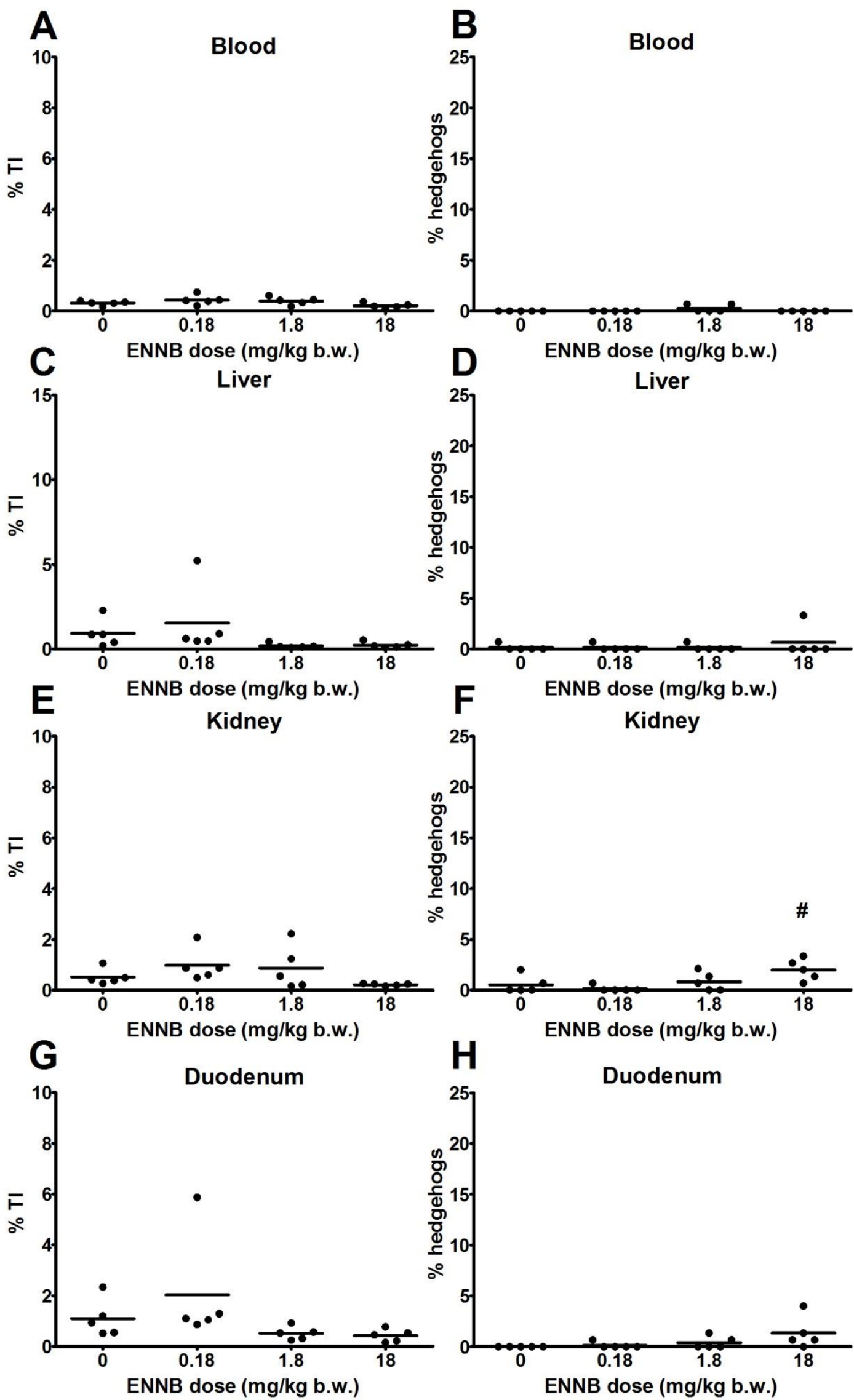

The present document has been produced and adopted by the bodies identified above as authors. In accordance with Article 36 of Regulation (EC) No $178 / 2002$, this task has been carried out exclusively by the authors in the context of a grant agreement between the European Food Safety Authority and the authors. The present document is published complying with the transparency principle to which the Authority is subject. It cannot be considered as an output adopted by the Authority. The European Food Safety Authority reserves its rights, view and position as regards the issues addressed and the conclusions reached in the present document, without prejudice to the rights of the authors. 

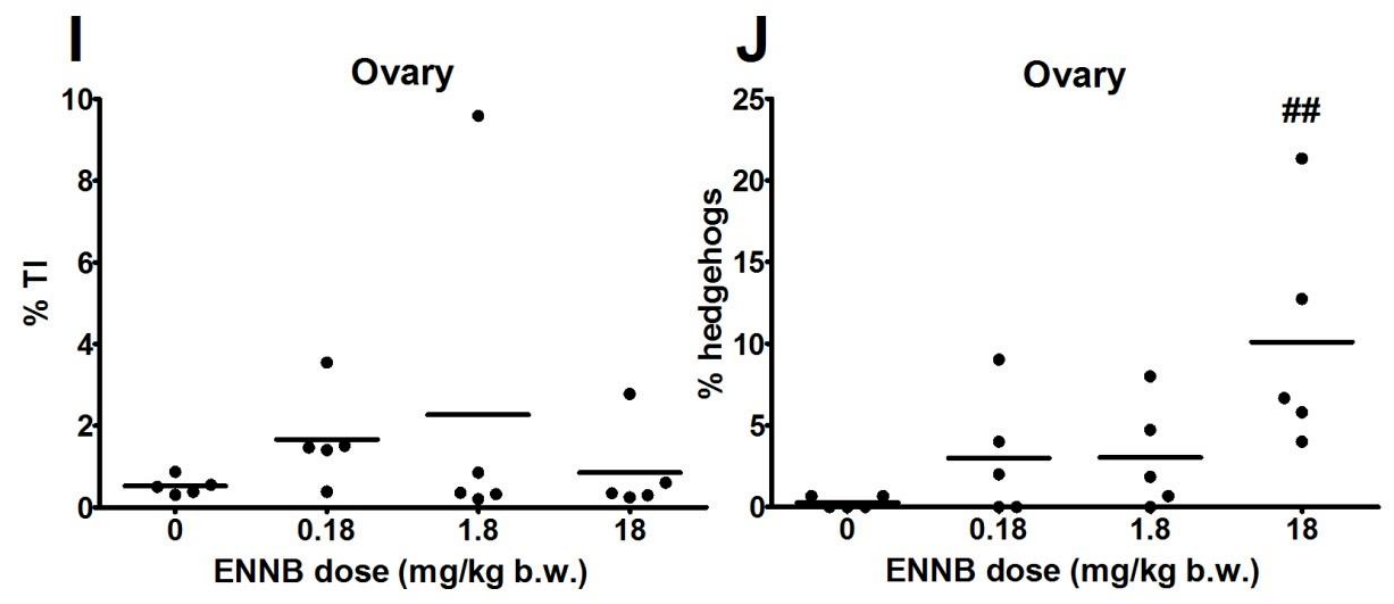

Figure 87: Alkaline Comet assay in different organs/tissues in dams after repeated oral treatments with different doses of ENNB ( 0 - olive oil+DMSO6\%; $0.18,1.8$ and $18 \mathrm{mg} / \mathrm{kg}$ per day). In A, C, E, G and I dots represent the median of tail intensity obtained for each animal and lines represent the mean of tail intensity for each group. In $B, D, F, H$ and J dots represent the percentage of hedgehogs obtained for each mouse and lines are the mean for each group; \# $\mathrm{p}<0.05 ; \# \# \mathrm{p}<0.01$. 


\section{Overall discussion}

\section{BEA}

For the repeated-dose study, BEA has been dissolved in olive oil and DMSO1.5\% (see Solubility Protocol). The BEA experiment has been paired with a CTRL group treated with olive oil only (negative CTRL). Concerns have arisen on the potential toxic effects of DMSO that could have had an impact on BEA results. Indeed, potential DMSO effect would have been evident in all dose groups. Since this was not the case this issue should not be critical for result interpretation, in particular for effects driving the study NOAEL definition.

It should be noted that for several tissues (e.g., enterocytes vacuolization in the duodenum, spleen histomorphometrical analysis), of the BEA experiment, the effects recorded were statistically different from the paired CTRL group without a clear dose-response relationship. In general, considering the size of the experimental groups and the mice inter-individual variability, it is not surprising to observe wide standard deviations for some of the endpoints analyzed. Since data distribution was not Gaussian, a non parametric analysis based on ranks has been performed which is most conservative and allows to avoid to obtain false positives. The statistical analysis and the biological interpretation are two distinct steps of the scientific evaluation of data: if only the middle and/or low dose level is significantly different from CTRL, this is a statistical/mathematical fact; the elucidation of the biological significance of this outcome may, otherwise, require additional investigation on the mode of action which may give supports to the interpretation of the results. Indeed, for the BEA NOAEL definition only effects with a clear dose-response relationship have been considered.

The sub-chronic BEA treatment did not induce death both in male and female mice nor overt toxicity. In fact, the body weight gain and food consumption were unaltered during the treatment.

The thyroid appears to be target tissue of BEA exposure; in males, this is supported by histopathological changes - reduced colloid in the follicles (Table 18) - and by T4 alterations at both mid- and high dose groups (Fig. 25); pyknotic nuclei are also present at high dose group. In female mice, only pyknotic nuclei were significantly increased at high dose (Table 18). This can be considered as a direct toxic effect of BEA on thyroid cells. The pore shape of the cyclodepsipeptide core of BEA suggested its ionophoric behaviour. Experimental studies showed that BEA can promote the transport of mono- and divalent cations $\left(\mathrm{K}+, \mathrm{N}+\right.$ and $\left.\mathrm{Ca}_{2}+\right)$ through membranes by creating pores. Data suggested that in the membrane BEA can act also as ion carrier, leading to toxic actions via disturbances in the normal physiological ion concentrations. It is known that $\mathrm{Ca}_{2}+$ stimulates cyclic AMP (cAMP) synthesis. It causes resorption of thyroid colloid and increases the rates of both glucose metabolism and protein synthesis as secretion of thyroid hormones increases in response to it (Zoeller, et al. 2007). Such mechanism might account for the BEA activity on thyroid gland of male mice. Since in females significant effect is present at BEA10 only, it can be hypothesized a different susceptibility between males and females.

Adrenals showed cortical vacuolization in female mice at all dose levels even though without a clear dose-response curve, due to the different severity. Adrenal cortical vacuolization in rodents is characterized by the accumulation of clear vacuoles within cortical cells, mainly in the zona fasciculata. Such effect showed a clear gender specificity since it wasn't recorded in male mice (Table 18).

Histomorphometrical alterations were present in spleen of male mice only: both the white and red pulp areas were increased at all dose levels although without a clear dose-response curve since the statistical significance was present at BEA0.1 and BEA10 (white pulp) and at BEA0.1 only (red pulp). No similar effects were recorded in female mice (Fig. 18).

Brains and thymus in both sexes and mammary glands were not affected by the treatment at any dose level (Table 18).

No effects were seen in liver and heart at any dose level in both sexes concerning tissue alterations (Table 18), and functional biomarkers for liver (Fig. 24). Relative weights of both tissues were significantly decreased in males at BEA10. (Table 17). 
Increased CRE serum levels without histopathological changes in kidneys were observed in male mice treated with BEA1 accompanied by decreased absolute weight (Table 17, Table 18 and Fig. 24). Kidney weight was altered at all dose levels without a clear dose-response curve (Table 17).

Pathological changes consisting of enterocytes vacuolization in the duodenum were observed in BEA1 females, the correlation with the treatment is unclear (Table 18)

BEA treatment showed several immunological effects in female mice. In particular, both frequency and functional activity (IFN $\gamma$ and IL-10, cytokines production, Fig. 30) of T cells in the spleen, considered as a central district, were upregulated at two doses (BEA0.1 and BEA10). However, no dose-response relationship could be observed. On the other hand, the innate immunity function (NO production, Fig. 29) was downregulated at the highest treatment dose. Conversely, in male mice, the only significant effect observed in the spleen cells was the increase of NO production at the highest dose. These effects on NO production induced by BEA treatment in female and male mice could be associated to the significantly higher NO production observed at the basal level (CTRL group) in females. Pro-inflammatory effects of oestrogens through their actions on the innate immune system have been described, probably accounting for the higher response to an inflammatory stimulus (LPS) by spleen macrophages in female mice (Hox et al, 2014). At the peripheral level (blood), only GRAN population was significantly reduced in male mice (Fig. 28).

Considering the female reproductive system, histopathological alterations in uteri were not accompanied by sexual steroid modifications. At BEA1 only, the presence of endometrial cysts was significantly increased together with decreased myometrial area and significantly decreased $T$ serum levels. At BEA10 the endometrial hyperplasia is significantly present, indicating a potential direct effect of BEA on endometrial component of the uterus (Table 18, Fig. 21 and Fig. 25).

Male reproductive system showed increased atrophic and with-germ-cell-disorganization tubules at BEA10 (Table 18). However, no decrease of sperm count was observed. No other biomarker effects were present at any dose level. At the highest dose level, BEA exposure induces tissue alterations in testes.

The screening of reproductive and developmental toxicity showed that BEA did not induce overt maternal toxicity since body weight gain and food consumption did not show differences among groups (Fig. 59 and Fig. 60).

Concerning reproductive or developmental effects, no statistically significant differences among groups were present for: time-to-pregnancy, the number of corpora lutea in uteri, the number of implants, death foetuses at birth, litter weight at birth and at PND 4, sex ratio (Fig. 61 and Fig. 62).

In dams, thymus absolute and relative weight was significantly increased at BEA1 and BEA10 without any histopathological alterations. Similar situation is present in the ovary, where the weight was significantly increased at BEA10 without histological alterations (Table 31 and Table 32).

The relevant pathological changes noted in treated dams, when compared with CTRLs, were increased severity of EMH in the spleen in all dams. Indeed, EMH normally occurs during pregnancy being a physiological process, either to support rapid expansion of maternal blood volume or necessary for fetal survival (Table 32).

Thyroid appeared to be affected by the treatment also in dams, since histopathological analysis showed increased follicular degeneration (Table 32), decreased number of follicles and follicular density at BEA 10 (Fig. 65). TSH serum levels were significantly increased at BEA0.1 only (Fig. 71).

Concerning the genotoxicity assays, the in vitro studies were negative, only MN formation was observed at the highest concentration in HepaRG cells (Table 16). The results of acute genotoxicity assessment by Comet assay in different organs (Fig. 3) and MN tests in bone marrow (Table 8) and colon (Fig. 6) indicated that no DNA damage was observed, whatever the organs/tissues observed up to $200 \mathrm{mg} / \mathrm{kg}$ b.w.. Although the MTD was not reached, the $200 \mathrm{mg} / \mathrm{kg}$ b.w. dose largely exceeded the highest acute human exposure estimates for BEA $(0.05 \mu \mathrm{g} / \mathrm{kg}$ b.w. per day (mean) and 0.10 
$\mu \mathrm{g} / \mathrm{kg}$ b.w. per day (95th percentile) (EFSA, 2014). For this reason, the negative results obtained can be considered informative for human risk assessment.

Regarding genotoxicity assessment after repeated oral exposure, the only statistically significant effects observed were the increased \%TI levels in kidney and duodenum of male mice treated with BEA1, while no effect was observed at BEA10 (Fig.32, Fig. 34 and Fig. 73). Overall, Comet results were reliable because of the homogeneity of Comet parameters in male and female CTRL groups and the low percentage of hedgehogs obtained in all organs analyzed. Although olive oil alone could not be considered the most appropriate vehicle to treat the CTRL group since $1.5 \%$ DMSO was necessary to dissolve BEA (see paragraph 2.2.2 Solubility protocols), the results can be considered informative for hazard characterization since any possible DMSO effect should have been likely present in all BEA treated groups, and this was not the case.

According to these results, the compound cannot be considered clearly genotoxic, since not all the criteria listed in OECD TG $489^{1}$ are fulfilled. In particular, there is no dose-related increase. Results obtained with Pig-a assay and MN test suggest that BEA does not induce gene mutations in bone marrow precursors of red blood cells and is not clastogenic or aneugenic in the same cells. Literature data on BEA genotoxicity derive exclusively from in vitro studies and report controversial results. BEA was non-mutagenic in Ames test using different Salmonella typhimurium strains, either with or without metabolic activation (Fotso and Smith, 2003). No genotoxic effects assessed by Comet assay were observed in two human cancer cell lines (Dornetshuber et al. 2009). A dose dependent induction of chromosomal aberrations, micronuclei and sister chromatid exchanges was observed after treatment of human lymphocytes (Çelik et al. 2010). A genotoxic effect was also demonstrated, by MN and Comet assay in porcine kidney epithelial cells PK15 and in human leukocytes with a different sensitivity (Klaric et al. 2008, 2010). CHO-K1 cells treated with different concentrations of BEA showed an increase of DNA damage as assessed by Comet assay, which was not dose dependent (Mallebrera et al. 2016). Possible mechanisms of BEA DNA damage induction have been investigated in a study (Dornetshuber et al. 2009) and the capacity of BEA to intercalate into the double helix was demonstrated but only at cytotoxic concentrations; an inhibition of topoisomerases activity was also shown at high concentrations. The same study also excluded a BEA induced increase of ROS. Overall, the authors concluded that BEA cytotoxicity prevailed over genotoxicity. On the other hand, a concentration-dependent increase in ROS production and lipid peroxidation was observed in $\mathrm{CHO}-\mathrm{K} 1$ cells (Ferrer et al. 2009).

Altogether, considering that:

- the in vitro genotoxicity studies were largely negative, only MN formation was observed at the highest concentration in HepaRG cells,

- no DNA damage was induced by acute exposure up to doses largely exceeding the actual human exposure,

- after repeated dose exposure no induction of gene mutation and chromosomal damage in bone marrow precursors of red blood cells or DNA damage in different organs analyzed were observed with the exception of kidney and duodenum of male mice in which an increase of damage was observed at BEA1 only,

it can be concluded that BEA has a low genotoxic potential; further investigations may be necessary under a cautionary approach.

\footnotetext{
${ }^{1}$ OECD, Test No. 489, 2014: In Vivo Mammalian Alkaline Comet Assay, OECD Publishing, Paris. 


\section{ENNB}

The repeated ENNB treatment did not induce death both in male and female mice. At ENNB18 body weight gain was decreased both in male and female mice; food consumption was increased in males only (Fig. 37).

In duodenum of males at ENNB18 enterocytes vacuolization was present. It was morphologically characterized by a progressive enlargement of vacuole as cells migrate from villus base to tip. This effect appears to be sex-specific since it was not present in female mice (Table 25).

In spleen and thymus of female mice, comparable pattern of effects was present with no concurrent alteration in absolute or relative organ weight; in spleen, both red and white pulp were increased at ENNB18, white pulp also at ENNB1.8 (Fig. 41). Thymus cortex and medulla areas were increased at ENNB18, cortex area also at ENNB1.8 (Fig. 42).

In adrenals of female mice, cortex area was increased and medulla was reduced at ENNB18 (Fig. 43). Adrenal gland is composed by two morphologically and functionally different part: cortex which produce mineralocorticoids, glucocorticoids and steroid hormones; medulla which produces epinephrine, norepinephrine, and a small amount of dopamine. In this respect, ENNB seems to exert selective effect on adrenal medulla in female mice. No effect in male mice was present.

In brain, relative weight was increased in males of all treatment groups and in females at ENNB18 (Table 24). ROS and GSH levels were increased in male mice, GSH also in females at ENNB18 (Fig. 48); such effects were not accompanied by histopathological changes (Table 25).

Kidney, mammary gland and MLN are not target of ENNB treatment.

As regards the immunotoxicity evaluation, ENNB effects were more scattered along all the analyzed endpoints and not selectively associated to one gender. The treatment induced significant effects in female mice mainly at the peripheral level, with the decrease of both circulating LYMPH and MID cells (Fig. 50), and the increase in IgG and IgA isotypes levels (Fig. 49). On the other hand, in the spleen only NO production after in vitro stimulation with LPS was increased (Fig. 51). In male mice, similar effects were induced by ENNB on circulating LYMPH and MID cells at the same treatment doses of female mice (Fig. 50), and similar effects were observed in spleen, where both the IL-10 secretion (Fig. 52) and the number of CD3/CD8+ T cells levels (Fig. 53) were decreased.

Concerning the female reproductive system, both myometrium and endometrium areas were increased at ENNB1.8 and ENNB18. Dilatation of the lumen was also present, whose area was significantly increased at ENNB1.8 only (Fig. 44). No absolute and/or relative tissue weight differences with concurrent CTRL+DMSO6\% were present (Table 24). No effects on hormone serum levels were present (Fig. 47).

The histopathological evaluation of ovaries was not possible due to the small number of ovaries, maybe lost during the sampling procedures.

Concerning the reproductive systems, female mice appeared to be more susceptible to ENNB treatment than males.

Overall the data showed that most of ENNB tissue effects are present in female mice. Immunotoxic data did not show such behavior.

The screening of reproductive and developmental toxicity showed that ENNB did not induce overt maternal toxicity since body weight gain and food consumption did not show differences among groups (Fig. 74 and Fig. 75).

Both absolute and relative weight of MLN were altered at ENNB1.8, absolute and relative heart weight was altered at ENNB1.8 and relative heart weight was altered at ENNB0.18 (Table 34) without any tissue damage (Table 35).

The relevant pathological changes noted in treated dams, when compared with CTRL, were increased severity of EMH in the spleen in all dams (Table 35). Moreover, at ENNB18 the white pulp was 
increased and red pulp/white pulp ratio was decreased (Fig. 81). Indeed, EMH normally occurs during pregnancy being a physiological process, either to support rapid expansion of maternal blood volume or necessary for fetal survival.

No significant alterations were present in biomarkers of liver and kidney function and in hormone serum levels (Fig. 84 and Fig. 85). Only ROS in brain was significantly decreased at ENNB1.8 (Fig. 86). The correlation with the treatment was unclear.

Concerning reproductive or developmental effects, no statistically significant differences among groups were present for: time-to-pregnancy, the number of corpora lutea in uteri, the number of implants, death foetuses at birth, litter weight at birth and at PND 4, sex ratio.

Concerning the genotoxicity assays, the Comet assay after acute exposure, when more sensitive electrophoretic conditions were applied, showed that ENNB induced a dose-dependent increase of DNA damage in liver and bone marrow (Table 12). Bone marrow MN test with ENNB was negative (Table 14). Therefore, the DNA damage observed by Comet assay in bone marrow seems not to be converted into MN formation. Similar results were obtained in the in vitro studies in which an induction of $\mathrm{YH} 2 \mathrm{AX}$ in HepaRG cells was observed, demonstrating the production of DNA strand breaks (Fig. 12). No formation of micronuclei after a short-time exposure to ENNB was observed in the same cells or in TK6 cell line with and without S9 mix (Table 15 and Table 16). Taken together our results show that ENNB induced genotoxic effects in liver and bone marrow, as assessed by Comet assay, after in vivo oral exposure in male mice.

Regarding genotoxicity assessment after repeated oral exposure, alkaline Comet assay results after ENNB treatment in male, female and dams showed that the compound did not significantly increase the \%TI in any of the organs analyzed indicating that ENNB, at the tested concentrations, was not able to induce DNA damage detectable by the Comet assay in these organs (Fig. 54, Fig. 56 and Fig. 87). Similarly, no induction of DNA damage was observed in epididymal spermatozoa analyzed by neutral Comet assay (Fig. 55). A significant increase of \% hedgehogs in ovary of dams was observed at the highest ENNB dose. The etiology of hedgehogs is uncertain and still debated. It has been suggested that they can be caused by substance-related cytotoxicity, mechanical-induced damage initiated during sample preparation and/or an extreme effect of test chemical genotoxicity (OECD TG 489'; Burlinson et al., 2007; Rothfuss et al., 2010; Kang et al., 2013). Since neither changes in ovary weights of ENNB treated dams were observed nor histopathological alterations were detected in the two dams that could be screened for this endpoint, the increase of \% hedgehogs was not to be due to cytotoxicity.

Results obtained with Pig-a assay and MN test suggest that ENNB, under the applied treatment protocol, did not induce gene mutations in bone marrow precursors of red blood cells and was not clastogenic or aneugenic in the same cells (Fig. 57 and Fig. 58).

Taken together the results of acute and repeated-dose in vivo genotoxicity tests support a tissuespecific genotoxic effect of ENNB after acute exposure to high doses.

\section{Overall conclusions}

Comparing the effects of BEA and ENNB at tissue/biomarker level, BEA targeted thyroid, kidneys and reproductive systems in both sexes whereas spleen is affected in male mice only and adrenals and duodenum in female mice only. Overall for these endpoints, taking into account also the severity of the effects, male mice seems more susceptible to BEA repeated oral exposure.

ENNB treatment affected spleen, brain and thyroid in both sexes whereas thymus, kidneys, adrenals and reproductive system in female mice only, duodenum in male mice only. Overall for these endpoints, taking into account also the severity of the effects, female mice seems more susceptible to ENNB repeated oral exposure.

Thyroid, adrenals are target of both mycotoxins in male and/or female mice; they belong to the endocrine system and - in this respect - their impact on the whole organism above all during critical

${ }^{1}$ OECD, Test No. 489, 2014: In Vivo Mammalian Alkaline Comet Assay, OECD Publishing, Paris. 
lifestages is well known. Although the reproductive/developmental screening with both BEA and ENNB didn't show any effects on the offspring, further and more specific studies should be considered to elucidate potential endocrine disrupting mechanisms.

As regards the global evaluation of immunotoxicity, it appears that the two mycotoxins BEA and ENNB induce different effects, mainly associated to the gender, to the immune districts affected and to the number of endpoints significantly altered. In particular, the effects of BEA treatment appeared more evident in female mice in which they were concentrated in the spleen with a prevalent positive action on the number and functional activity of effector T cells. Otherwise, ENNB effects were observed in both genders, and they were more suggestive of a suppressive/inhibiting activity particularly evident in male mice. Interestingly, parallel enhancing effects of the two mycotoxins on the innate compartment of the immune system (NO production by adherent spleen cells) have been observed with the only exception of BEA-treated female mice in which an inhibitory action was recorded. Notably, both the mycotoxins did not affect the RBC and PLT populations, suggesting the absence of effects in the haemotoxicity and coagulation compartments, respectively. On the other hand, the effects of the two mycotoxins on the reduction of immunity-related blood cell populations (e.g.: lymphocytes, granulocytes, monocyte-like) could be related to their myelotoxicity potential, observed in vitro on human hematopoietic progenitors.

A possible explanation of the different pattern of effects induced by BEA and ENNB (central vs. peripheral effects, respectively) could be associated to the different metabolism of the two mycotoxins. In fact, despite their structural similarities, while for BEA no metabolites have been detected, ENNB metabolites have been found in liver and colon, thus potentially contributing in different modes to the biodistribution and the toxic effects (Rodriguez-Carrasco et al., 2016).

The immunotoxicity results obtained in the present in vivo study show interesting analogies with in vitro studies performed on the murine macrophage cell line RAW264.7 exposed to ENNB, in which an increased release of inflammatory cytokines (IL-1B) was observed upon priming with LPS (Gammelsrud et al., 2012).

\section{BEA}

All the genotoxicity endpoints analyzed in the acute and repeated dose studies yielded negative results with the exception of Comet assay in duodenum and kidney but without a dose-related effect. Concluding, BEA has a low genotoxic potential; further investigations may be necessary under a cautionary approach.

Considering the overall results of the repeated dose BEA study, the NOAEL for female mice is $1 \mathrm{mg} / \mathrm{kg}$ b.w. per day based on increased thyroid pycnotic nuclei and endometrial hyperplasia. In male mice, the NOAEL is $0.1 \mathrm{mg} / \mathrm{kg} \mathrm{b.w.} \mathrm{per} \mathrm{day} \mathrm{based} \mathrm{on} \mathrm{reduced} \mathrm{colloid} \mathrm{and} \mathrm{altered} \mathrm{T4} \mathrm{serum} \mathrm{levels} \mathrm{(Table} 18$ and Fig. 25).

Considering the screening of reproductive and development study, the maternal NOAEL is $0.1 \mathrm{mg} / \mathrm{kg}$ b.w. due to the increased absolute and relative thymus weight and increased follicle degeneration (Table 31). Developmental NOAEL is $10 \mathrm{mg} / \mathrm{kg} \mathrm{b.w.} \mathrm{per} \mathrm{day.}$ 
Table 37: NOAELS/LOAELs of BEA after repeated oral administration in mice

\begin{tabular}{|c|c|c|c|c|c|}
\hline Type of NOAEL & $\begin{array}{c}\text { Dose/ } \\
\text { duration }\end{array}$ & Sex & $\begin{array}{c}\text { NOAEL } \\
(\mathrm{mg} / \mathrm{kg} \text { b.w./day })\end{array}$ & $\begin{array}{c}\text { LOAEL } \\
(\mathrm{mg} / \mathrm{kg} \text { b.w./day })\end{array}$ & $\begin{array}{l}\text { Effect at the } \\
\text { LOAEL }\end{array}$ \\
\hline \multirow{2}{*}{$\begin{array}{l}\text { Sub-chronic } \\
\text { toxicity }\end{array}$} & \multirow{2}{*}{$\begin{array}{l}5 \text { days/week, } \\
42 \text { days by } \\
\text { oral gavage }\end{array}$} & Male & 0.1 & (2) & $\begin{array}{c}\text { Reduced colloid } \\
\text { in thyroid/ } \\
\text { altered T4 } \\
\text { serum levels } \\
\end{array}$ \\
\hline & & Female & 1 & 10 & $\begin{array}{c}\text { Thyroid pycnotic } \\
\text { nuclei/endometri } \\
\text { al hyperplasia }\end{array}$ \\
\hline \multirow[t]{2}{*}{$\begin{array}{l}\text { Reproductive } \\
\text { and } \\
\text { developmental } \\
\text { study }\end{array}$} & \multirow[t]{2}{*}{$\begin{array}{l}5 \text { days/week, } \\
42 \text { days by } \\
\text { oral gavage }\end{array}$} & Dams & 0.1 & 1 & $\begin{array}{c}\text { Increased } \\
\text { absolute and } \\
\text { relative thymus } \\
\text { weight and } \\
\text { increased follicle } \\
\text { degeneration }\end{array}$ \\
\hline & & Offspring & 10 & Not applicable & No effect \\
\hline
\end{tabular}




\section{ENNB}

Based on both the repeated and the acute treatment studies, results support the conclusion that ENNB poses a genotoxic hazard.

Considering the overall results of the repeated-dose ENNB study, the NOAEL for female mice is 0.18 $\mathrm{mg} / \mathrm{kg}$ b.w. per day based on the dose-related histomorphometrical effects on thymus, uterus and spleen (Fig. 41, Fig. 42 and Fig. 43). In male mice, the NOAEL is $1.8 \mathrm{mg} / \mathrm{kg} \mathrm{b.w.} \mathrm{per} \mathrm{day} \mathrm{based} \mathrm{on}$ enterocyte vacuolization in duodenum (Table 25) and increased ROS and GSH brain levels at the highest dose tested (Fig. 48).

Considering the screening of reproductive and development study, the maternal NOAEL is $1.8 \mathrm{mg} / \mathrm{kg}$ b.w. per day due to the increased white pulp area and decreased red/white pulp area ratio in spleen (Fig. 81). Developmental NOAEL is $18 \mathrm{mg} / \mathrm{kg}$ b.w. per day.

Table 38: NOAELS/LOAELs of ENNB after sub-chronic oral administration in mice

\begin{tabular}{|c|c|c|c|c|c|}
\hline Type of NOAEL & Dose/duration & Sex & $\begin{array}{c}\text { NOAEL } \\
(\mathrm{mg} / \mathrm{kg} \mathrm{b.w./day})\end{array}$ & $\begin{array}{c}\text { LOAEL } \\
\text { (mg/kg } \\
\text { b.w./day) }\end{array}$ & $\begin{array}{l}\text { Effect at the } \\
\text { LOAEL }\end{array}$ \\
\hline \multirow{2}{*}{$\begin{array}{l}\text { Sub-chronic } \\
\text { toxicity }\end{array}$} & \multirow{2}{*}{$\begin{array}{c}5 \text { days/week, } \\
42 \text { days by } \\
\text { oral gavage }\end{array}$} & Male & 1.8 & 18 & $\begin{array}{c}\text { Enterocyte } \\
\text { vacuolization/Inc } \\
\text { reased ROS and } \\
\text { GSH brain levels }\end{array}$ \\
\hline & & Female & 0.18 & 1.8 & $\begin{array}{c}\text { Histomorphomet } \\
\text { rical effects on } \\
\text { thymus, uterus, } \\
\text { spleen }\end{array}$ \\
\hline \multirow[t]{2}{*}{$\begin{array}{l}\text { Reproductive } \\
\text { and } \\
\text { developmental } \\
\text { study }\end{array}$} & \multirow[t]{2}{*}{$\begin{array}{c}5 \text { days/week, } \\
42 \text { days by } \\
\text { oral gavage }\end{array}$} & Dams & 1.8 & 18 & $\begin{array}{l}\text { Increased white } \\
\text { pulp area and } \\
\text { decreased } \\
\text { red/white pulp } \\
\text { area ratio in } \\
\text { spleen } \\
\end{array}$ \\
\hline & & Offspring & 18 & Not applicable & No effect \\
\hline
\end{tabular}




\section{References}

Bernacki DT, Bryce SM, Bemis JC, Kirkland D and Dertinger SD, 2016. yH2AX and p53 responses in TK6 cells discriminate promutagens and nongenotoxicants in the presence of rat liver S9. Environmental and Mololecular Mutagenesis, 57, 546-558.

Bowen DE, Whitwell JH, Lillford L, Henderson D, Kidd D, Mc Garry S, Pearce G, Beevers C and Kirkland DJ, 2011. Evaluation of a multi-endpoint assay in rats, combining the bone-marrow micronucleus test, the Comet assay and the flow-cytometric peripheral blood micronucleus test. Mutation Research, 722, 7-19.

Bryce SM, Bernacki DT, Bemis JC, Spellman RA, Engel ME, Schuler M, Lorge E, Heikkinen PT, Hemmann U, Thybaud V, Wilde S, Queisser N, Sutter A, Zeller A, Guérard M, Kirkland D and Dertinger SD, 2017. Interlaboratory evaluation of a multiplexed high information content in vitro genotoxicity assay. Environmental and Mololecular Mutagenesis, 58, 146-161.

Burlinson B, Tice RR, Speit G, Agurell E, Brendler-Schwaab SY, Collins AR, Escobar P, Honma M, Kumaravel TS, Nakajima M, Sasaki YF, Thybaud V, Uno Y, Vasquez M, Hartmann A; In Vivo Comet Assay Workgroup, part of the Fourth International Workgroup on Genotoxicity Testing, 2007. Fourth International Workgroup on Genotoxicity testing: results of the in vivo Comet assay workgroup. Mutation Reseach, 3, 31-35.

Çelik M, Aksoy $\mathrm{H}$ and Yılmaz S, 2010. Evaluation of Beauvericin genotoxicity with the chromosomal aberrations, sister-chromatid exchanges and micronucleus assays. Ecotoxicology and Environmental Safety, 73, 1553-1557.

Dertinger, S D, Bryce, S M, Phonethepswath, S and Avlasevich, S L, 2011. When Pigs Fly: Immunomagnetic Separation Facilitates Rapid Determination of Pig-a Mutant Frequency by Flow Cytometric Analysis. Mutation Research, 721, 163-170.

Dornetshuber R, Heffeter P, Lemmens-Gruber R, Elbling L, Marko D, Micksche M and Berger W, 2009. Oxidative stress and DNA interactions are not involved in enniatin- and beauvericin-mediated apoptosis induction. Molecular Nutrition \& Food Research, 53, 1112-1122.

EFSA CONTAM Panel (EFSA Panel on Contaminants in the Food Chain), 2014. Scientific Opinion on the risks to human and animal health related to the presence of beauvericin and enniatins in food and feed. EFSA Journal 12(8):3802, 174 pp. doi:10.2903/j.efsa.2014.3802

Fenech M, Chang WP, Kirsch-Volders M, Holland N, Bonassi S, Zeiger E and HUman MicronNucleus project, 2003. HUMN project: detailed description of the scoring criteria for the cytokinesis-block micronucleus assay using isolated human lymphocyte cultures. Mutation Research, 534, 65-75.

Ferrer E, Juan-García A, Font $G$ and Ruiz MJ, 2009. Reactive oxygen species induced by beauvericin, patulin and zearalenone in CHO-K1 cells. Toxicology In Vitro, 23, 1504-1509.

Fortune JE, 2003. The early stages of follicular development: activation of primordial follicles and growth of preantral follicles. Animal Reproduction Science, 78, 135-163.

Fotso J and Smith JS, 2003. Evaluation of beauvericin toxicity with the bacterial bioluminescence assay and the Ames mutagenicity bioassay. Journal of Food Science, 68, 1938-1941.

Gammelsrud A, Solhaug A, Dendelé B, Sandberg WJ, Ivanova L, Kocbach Bølling A, LagadicGossmann D, Refsnes M, Becher R, Eriksen G and Holme JA, 2012. Enniatin B-induced cell death and inflammatory responses in RAW 267.4 murine macrophages. Toxicology and Applied Pharmacology, 261, 74-87.

Godin-Ethier J, Leroux F, Wang N, Thébaud S, Merah F and Nelson A, 2015. Characterisation of an in vivo Pig-a gene mutation assay for use in regulatory toxicology studies. Mutagenesis, 30, 359-63.

Gollapudi BB, Lynch AM, Heflich RH, Dertinger SD, Dobrovolsky VN, Froetschl R, Horibata K, Kenyon MO, Kimoto T, Lovell DP, Stankowski LF Jr, White PA, Witt KL and Tanir JY, 2015. The in vivo Pig-a 
assay: A report of the International Workshop On Genotoxicity Testing (IWGT) Workgroup. Mutation Research - Genetic Toxicology and Environmental Mutagenesis, 783, 23-35.

Gunther WC, Coffing SL, Dickinson DA, Engel ME, Fiedler RD, O'Lone SD, Sanok KE, Thiffeault CJ, Shutsky TJ, Schuler MJ and Dobo KL, 2014. Evaluation of the Pig-a, micronucleus, and comet assay endpoints in a 28-day study with ethyl methanesulfonate. Environmental and Mololecular Mutagenesis, 55, 492-499.

Hayashi M, MacGregor JT, Gatehouse DG, Blakey DH, Dertinger SD, Abramsson-Zetterberg L, Krishna G, Morita T, Russo A, Asano N, Suzuki H, Ohyama W, Gibson D; In Vivo Micronucleus Assay Working Group, IWGT, 2007. In vivo erythrocyte micronucleus assay III. Validation and regulatory acceptance of automated scoring and the use of rat peripheral blood reticulocytes, with discussion of non-hematopoietic target cells and a single dose-level limit test. Mutation Research, 62, 10-30.

Hox V, Desai A, Bandara G, Gilfillan AM, Metcalfe DD and Olivera A, 2014. Estrogen increases the severity of anaphylaxis in female mice through enhanced endothelial nitric oxide synthase expression and nitric oxide production. Journal of Allergy and Clinical Immunology, 135, 729-36.

Juan C, Manyes J, Font G and Juan-García A, 2014. Evaluation of immunologic effect of Enniatin A and quantitative determination in feces, urine and serum on treated Wistar rats. Toxicon, 87, 45-53.

Kang SH, Kwon JY, Lee JK, Seo YR, 2013. Recent advances in in vivo genotoxicity testing: prediction of carcinogenic potential using comet and micronucleus assay in animal models. Journal of Cancer Prevention, 18, 277-288.

Klaric MS, Pepeljnjak S and Rozgaj R, 2008. Genotoxicity of fumonisin B1, beauvericin and ochratoxin A in porcine kidney PK15 cells: effects of individual and combined treatment. Croatica Chemica Acta, 81, 139-146.

Klaric MS, Darabos D, Rozgaj R, Kasuba V and Pepeljnjak S, 2010. Beauvericin and ochratoxin A genotoxicity evaluated using the alkaline comet assay: single and combined genotoxic action. Archives of Toxicology, 84, 641-650.

Khoury L, Zalko D and Audebert M, 2016. Complementarity of phosphorylated histones H2AX and H3 quantification in different cell lines for genotoxicity screening. Archives of Toxicology, 90, 19831995.

Mallebrera B, Juan-Garcia A, Font G and Ruiz MJ, 2016. Mechanisms of beauvericin toxicity and antioxidant cellular defense. Toxicology Letters, 246, 28-34.

Maranghi F, Tassinari R, Lagatta V, Moracci G, Macrì C, Eusepi A, Di Virgilio A, Scattoni ML and Calamandrei $G$, 2009. Effects of the food contaminant semicarbazide following oral administration in juvenile Sprague-Dawley rats. Food and Chemical Toxicology , 47, 472-479.

Miura D, Dobrovolsky VN, Kimoto T, Kasahara $Y$ and Heflich RH, 2009. Accumulation and persistence of Pig-A mutant peripheral red blood cells following treatment of rats with single and split doses of N-ethyl-N-nitrosourea. Mutation Research, 677, 86-92.

Omura S, Koda $\mathrm{H}$ and Nishida $\mathrm{H}, 1991$. Hypolipemics containing beauvericin as acylcoenzyme $A$ cholesterol acyltransferase inhibitor. Patent JP 89-16115019890623.

Rodriguez-Carrasco Y, Heilos D, Richter L, Süssmuth RD, Heffeter P, Sulyok M, Kenner L, Berger W and Dornetshuber-Fleiss $R, 2016$. Mouse tissue distribution and persistence of the food-born fusariotoxins Enniatin B and Beauvericin. Toxicology Letters, 247:35-44.

Rogakou EP, Pilch DR, Orr AH, Ivanova VS, Bonner WM, 1998. DNA double-stranded breaks induce histone H2AX phosphorylation on serine 139, J Biol Chem, 6, 5858-5868.

Rothfuss A, O'Donovan M, De Boeck M, Brault D, Czich A, Custer L, Hamada S, Plappert-Helbig U, Hayashi M, Howe J, Kraynak AR, van der Leede BJ, Nakajima M, Priestley C, Thybaud V, Saigo K, Sawant S, Shi J, Storer R, Struwe M, Vock E and Galloway S, 2010. Collaborative study on fifteen 
compounds in the rat-liver Comet assay integrated into 2- and 4-week repeat-dose studies. Mutation Research, 702, 40-69.

Shackelford C, Long G, Wolf J, Okerberg C and Herbert R, 2002. Qualitative and quantitative analysis of nonneoplastic lesions in toxicology studies. Toxicologic Pathology, 30, 93-96.

Zoeller RT, Tan SW and Tyl RW, 2007. General background on the hypothalamic-pituitary-thyroid (HPT) axis.; Critical Reviews in Toxicology, 37, 11-53. 


\section{Abbreviations}

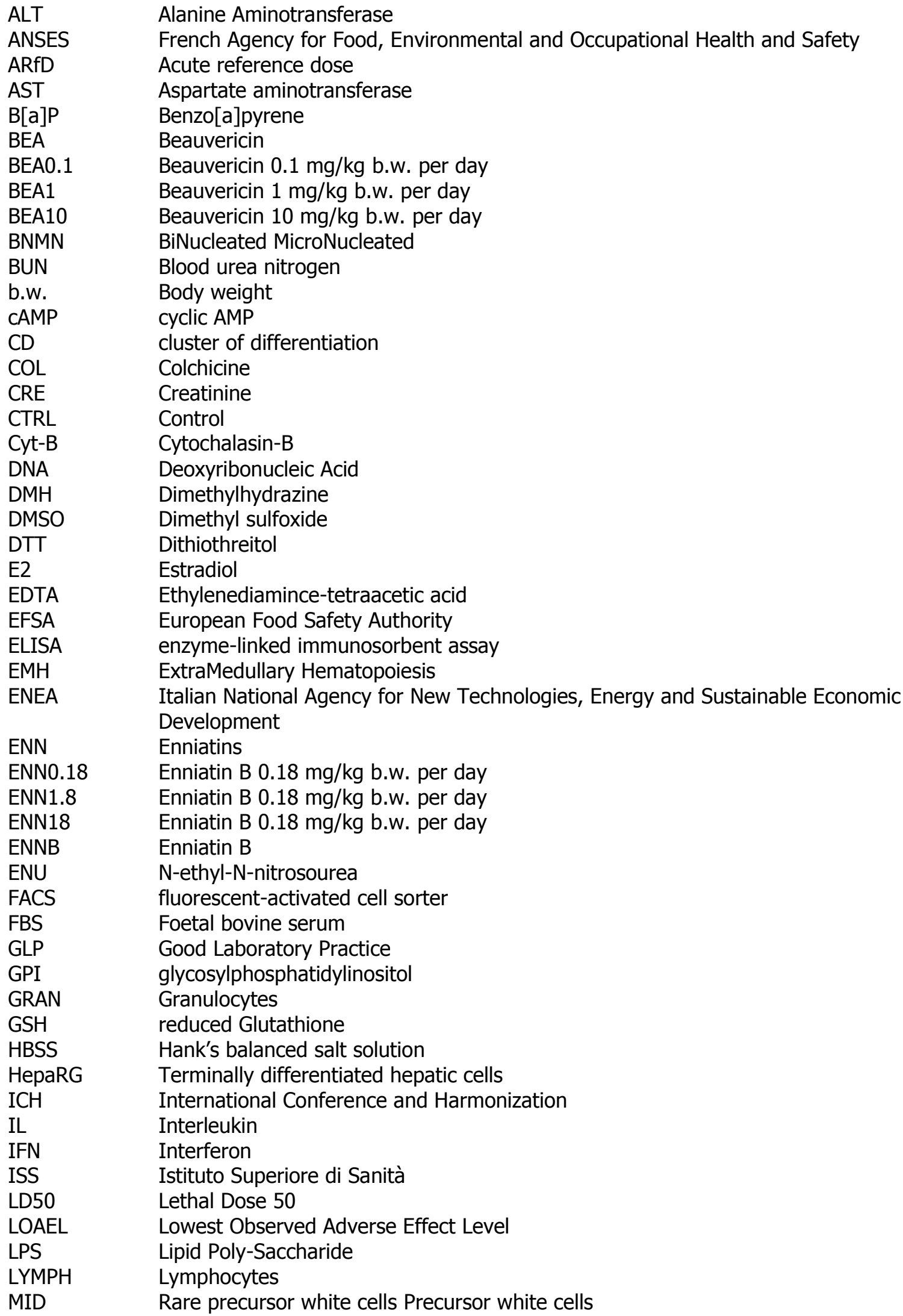

ALT ANSES

ARfD

AST

$\mathrm{B}[\mathrm{a}] \mathrm{P}$

BEA

BEA0.1

BEA1

BEA10

BNMN

BUN

b.w.

cAMP

CD

$\mathrm{COL}$

CRE

CTRL

Cyt-B

DNA

DMH

DMSO

DTT

E2

EDTA

EFSA

ELISA

EMH

ENEA

ENN

ENN0.18

ENN1.8

ENN18

ENNB

ENU

FACS

FBS

GLP

GPI

GRAN

GSH

HBSS

HepaRG

$\mathrm{ICH}$

IL

IFN

ISS

LD50

LOAEL

LPS

LYMPH

MID

Alanine Aminotransferase

French Agency for Food, Environmental and Occupational Health and Safety Acute reference dose

Aspartate aminotransferase

Benzo[a]pyrene

Beauvericin

Beauvericin $0.1 \mathrm{mg} / \mathrm{kg}$ b.w. per day

Beauvericin $1 \mathrm{mg} / \mathrm{kg}$ b.w. per day

Beauvericin $10 \mathrm{mg} / \mathrm{kg}$ b.w. per day

BiNucleated MicroNucleated

Blood urea nitrogen

Body weight

cyclic AMP

cluster of differentiation

Colchicine

Creatinine

Control

Cytochalasin-B

Deoxyribonucleic Acid

Dimethylhydrazine

Dimethyl sulfoxide

Dithiothreitol

Estradiol

Ethylenediamince-tetraacetic acid

European Food Safety Authority

enzyme-linked immunosorbent assay

ExtraMedullary Hematopoiesis

Italian National Agency for New Technologies, Energy and Sustainable Economic

\section{Development}

Enniatins

Enniatin B $0.18 \mathrm{mg} / \mathrm{kg}$ b.w. per day

Enniatin B $0.18 \mathrm{mg} / \mathrm{kg}$ b.w. per day

Enniatin B $0.18 \mathrm{mg} / \mathrm{kg}$ b.w. per day

Enniatin B

$\mathrm{N}$-ethyl-N-nitrosourea

fluorescent-activated cell sorter

Foetal bovine serum

Good Laboratory Practice

glycosylphosphatidylinositol

Granulocytes

reduced Glutathione

Hank's balanced salt solution

Terminally differentiated hepatic cells

International Conference and Harmonization

Interleukin

Interferon

Istituto Superiore di Sanità

Lethal Dose 50

Lowest Observed Adverse Effect Level

Lipid Poly-Saccharide

Lymphocytes

Rare precursor white cells Precursor white cells 


$\begin{array}{ll}\text { MLN } & \text { Mesenteric lymph node } \\ \text { MMS } & \text { Methylmethanesulfonate } \\ \text { MN } & \text { Micronucleus } \\ \text { MTD } & \text { Maximun Tolerated Dose } \\ \text { NCE } & \text { Normochromatic erythrocytes } \\ \text { NO } & \text { Nitric Oxide } \\ \text { NOAEL } & \text { No Observed Adverse Effect Level } \\ \text { OECD } & \text { Organisation for Economic Co-operation and Development } \\ \text { OTM } & \text { Olive Tail Moment } \\ \text { PBS } & \text { Phosphate Buffered Saline } \\ \text { PCE } & \text { Polychromatic erythrocytes } \\ \text { PLT } & \text { Platelets } \\ \text { PND } & \text { Post-natal day } \\ \text { RBCs } & \text { Red Blood Cell } \\ \text { RCC } & \text { Relative cell counts } \\ \text { RETs } & \text { Reticulocytes } \\ \text { RI } & \text { Replication Index } \\ \text { RICC } & \text { Relative increase in cell count } \\ \text { ROS } & \text { Reactive Oxidative Species } \\ \text { RPD } & \text { Relative population doubling } \\ \text { SE } & \text { Standard Error } \\ \text { T } & \text { Testosterone } \\ \text { T4 } & \text { Tetraiodothyronine } \\ \text { Tail-L } & \text { Tail length } \\ \text { TDI } & \text { Tolerable daily intake } \\ \text { TG } & \text { Test Guideline } \\ \text { TI } & \text { Tail intensity } \\ \text { TK6 } & \text { Lymphoblast thymidine kinase heterozygote cell line } \\ \text { TSH } & \text { Thyroid stimulating hormone } \\ \text { WBC } & \text { White blood cells } \\ \end{array}$


Appendix A - Timeline for in vivo genotoxicity tests integrated to repeat-dose toxicity study

- EXCEL file - Appendices available at EFSA

\section{Appendix B - $\quad$ Acute in vivo genotoxicity raw data BEA and ENNB}

- EXCEL file - Appendices available at EFSA

\section{Appendix C - In vitro genotoxicity raw data BEA and ENNB}

- EXCEL file - Appendices available at EFSA

\section{Appendix D - Sub-chronic and reproductive study raw data BEA}

- EXCEL file - Appendices available at EFSA

\section{Appendix E - Sub-chronic and reproductive study raw data ENNB}

- EXCEL file - Appendices available at EFSA 